\title{
Direct Synthesis of Enamides via Electrophilic Activation of Amides
}

Philipp Spieß, ${ }^{\mathrm{a}}$ Martin Berger, ${ }^{\mathrm{a}}$ Daniel Kaiser ${ }^{\mathrm{a}}$ and Nuno Maulide ${ }^{\mathrm{a} *}$

${ }^{a}$ Institute of Organic Chemistry, University of Vienna, Währinger Straße 38, 1090 Vienna, Austria

E-Mail: nuno.maulide@univie.ac.at, Homepage: http://maulide.univie.ac.at

\section{Table of Contents}

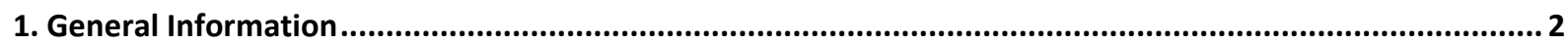

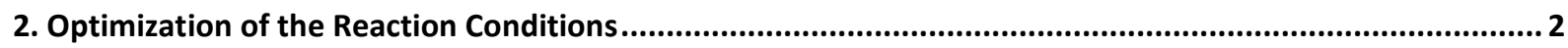

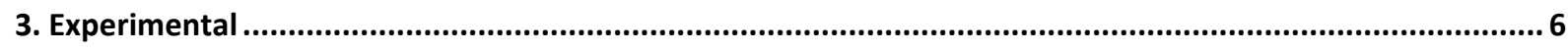

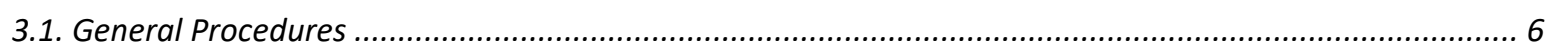

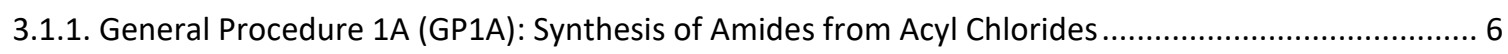

3.1.2. General Procedure 1B (GP1B): Synthesis of Amides from Acyl Chlorides ...................................... 6

3.1.3. General Procedure 2A (GP2A): Synthesis of Amides from Carboxylic Acids ................................. 6

3.1.4. General Procedure 2B (GP2B): Synthesis of Amides from Carboxylic Acids .................................. 7

3.1.5. General Procedure 3 (GP3): Synthesis of Enamides .............................................................. 7

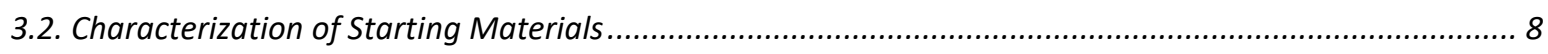

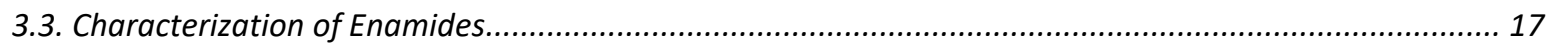

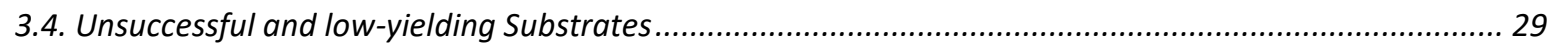

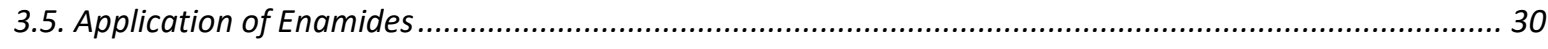

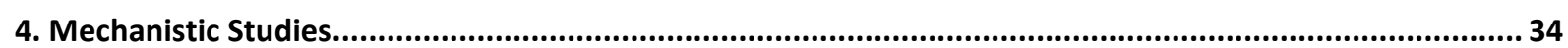

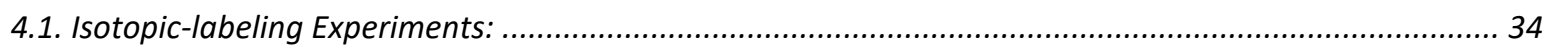

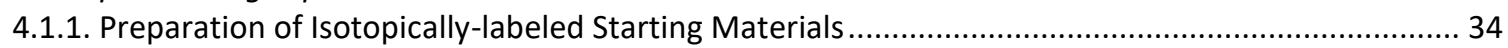

4.1.2. $N$-Dehydrogenation Reaction with Isotopically-labeled Substrates ........................................... 37

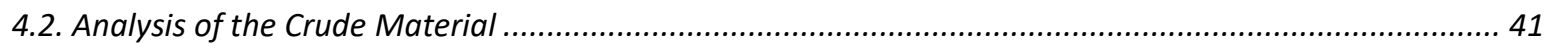

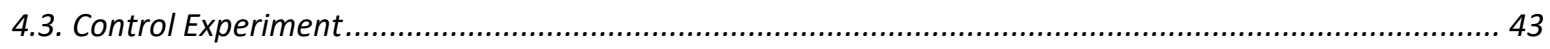

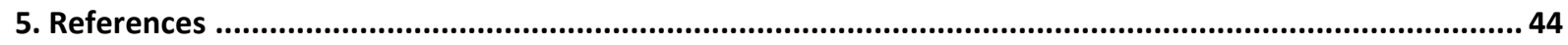

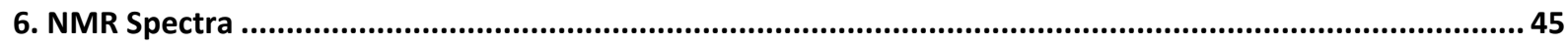

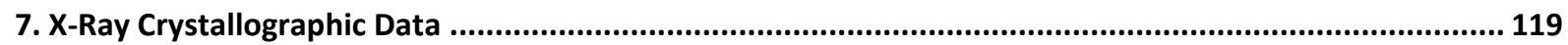




\section{General Information}

Unless otherwise stated, all glassware was flame-dried before use and all reactions were performed under an atmosphere of argon. All solvents were distilled from appropriate drying agents prior to use or directly taken from commercial sealed bottles under an atmosphere of argon. All reagents were used as received from commercial suppliers unless otherwise stated. Trifluoromethanesulfonic anhydride $\left(\mathrm{Tf}_{2} \mathrm{O}\right)$ was distilled over $\mathrm{P}_{4} \mathrm{O}_{10}$ prior to use and stored under inert atmosphere in the fridge for a maximum of roughly 3 weeks. ${ }^{[1]}$ Reaction progress was monitored by thin layer chromatography (TLC) performed on aluminum plates coated with silica gel F254 with $0.2 \mathrm{~mm}$ thickness. Chromatograms were visualized by fluorescence quenching with UV light at $254 \mathrm{~nm}$ or by staining using potassium permanganate. Flash column chromatography was performed using silica gel 60 (230-400 mesh, Merck and co.). Neat infrared spectra were recorded using a Perkin-Elmer Spectrum 100 FT-IR spectrometer. Wavenumbers $\left(v_{\max }\right)$ are reported in $\mathrm{cm}^{-1}$. Mass spectra were obtained using a Finnigan MAT 8200 or $(70 \mathrm{eV})$ or an Agilent $5973(70 \mathrm{eV})$ spectrometer, using electrospray ionization (ESI). All ${ }^{1} \mathrm{H}$ NMR and ${ }^{13} \mathrm{C}$ NMR spectra were recorded using a Bruker AV-400, AV-600 spectrometer or AV-700 spectrometer at $300 \mathrm{~K}$. Chemical shifts are given in parts per million (ppm, $\delta)$, referenced to the solvent peak of $\mathrm{CDCl}_{3}$, defined at $\delta=7.26 \mathrm{ppm}\left({ }^{1} \mathrm{H} \mathrm{NMR}\right)$ and $\delta=77.16\left({ }^{13} \mathrm{C} \mathrm{NMR}\right)$. Coupling constants are quoted in $\mathrm{Hz}(J) .{ }^{1} \mathrm{H}$ NMR splitting patterns are designated as singlet $(\mathrm{s})$, doublet (d), triplet (t), quartet (q) and quintet (quint) as they appeared in the spectrum. If the appearance of a signal differs from the expected splitting pattern, the observed pattern is designated as apparent (app). Splitting patterns that could not be interpreted or easily visualized are designated as multiplet $(\mathrm{m})$ or broad (br).

\section{Optimization of the Reaction Conditions}

General procedure: A flame-dried Schlenk flask was loaded with amide (1a, $69.4 \mathrm{mg}, 0.30 \mathrm{mmol}$, 1.00 eq.) and dissolved in the corresponding solvent. The solution was set to the desired temperature (temperature was kept until quenching) and the base was added slowly (20 s). After the addition, the resulting mixture was stirred for $10 \mathrm{~min}$. Following that, $\mathrm{Tf}_{2} \mathrm{O}$ was added dropwise over $1 \mathrm{~min}$ under vigorous stirring. The reaction was stirred for $30 \mathrm{~min}$ before remaining reagents were quenched by the addition of a saturated solution of $\mathrm{NH}_{4} \mathrm{Cl}(5 \mathrm{~mL})$. The yield was determined by $\mathrm{GC}$ FID analysis using 1-decane $(58.5 \mu \mathrm{L}, 42.7 \mathrm{mg}, 0.30 \mathrm{mmol})$ as internal standard.

Table S1. Optimization of the reaction temperature. ${ }^{a}$

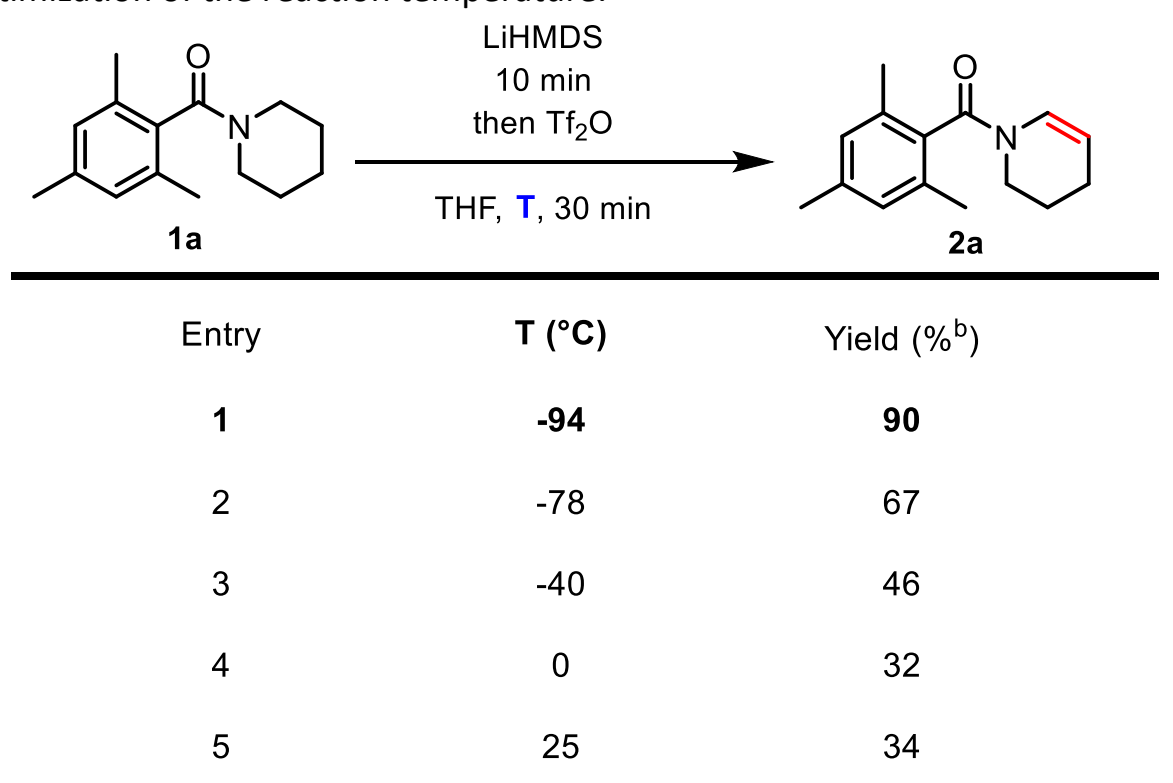

a 1a $(0.30 \mathrm{mmol})$, THF $(1.5 \mathrm{~mL})$, LiHMDS $\left(0.96 \mathrm{mmol}, 1 \mathrm{M}\right.$ solution in THF) with slow addition, $10 \mathrm{~min}$, then Tf $\mathrm{f}_{2} \mathrm{O}(0.48 \mathrm{mmol})$ with addition over 1 min. ${ }^{b} \mathrm{GC}$ yield. 
Table S2. Optimization of the base. ${ }^{a}$

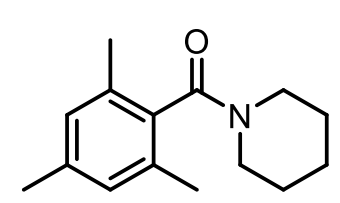

$1 \mathrm{a}$

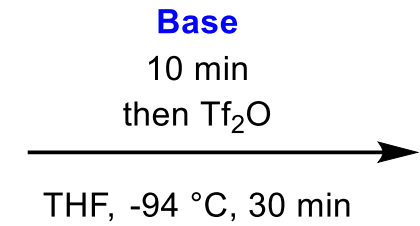

THF, $-94^{\circ} \mathrm{C}, 30 \mathrm{~min}$<smiles>Cc1cc(C)c(C(=O)N2C=CCCC2)c(C)c1</smiles>

$2 a$

$\begin{array}{ccc}\text { Entry } & \text { Base } & \text { Yield }\left(\%{ }^{\mathrm{b}}\right) \\ 1 & \text { LiHMDS }^{\mathrm{c}} & 90 \\ 2 & \text { NaHMDS }^{\mathrm{c}} & 33 \\ 3 & \text { KHMDS }^{\mathrm{C}} & 6 \\ 4 & \text { LiOtBu }^{\mathrm{c}} & 6 \\ 5 & \text { tBuLi }^{\mathrm{d}} & 0 \\ 6 & \mathrm{NaH}^{\mathrm{e}} & \text { traces }\end{array}$

${ }^{a}$ 1a $(0.30 \mathrm{mmol}), \operatorname{THF}(1.5 \mathrm{~mL})$, Base with slow addition, $10 \mathrm{~min}$, then $\mathrm{Tf}_{2} \mathrm{O}(0.48 \mathrm{mmol})$ with addition over $1 \mathrm{~min} .{ }^{\mathrm{b}} \mathrm{GC}$ yield. ${ }^{\mathrm{c}}$ bases were used as $1 \mathrm{M}$ solutions in THF. ${ }^{\mathrm{d}} 1.7 \mathrm{M}$ in pentane. ${ }^{\mathrm{e}}$ was added neat.

Table S4. Optimization of the used equivalents of reagents. ${ }^{a}$<smiles>Cc1cc(C)c(C(=O)N2CCCCC2)c(C)c1</smiles>

$1 \mathrm{a}$

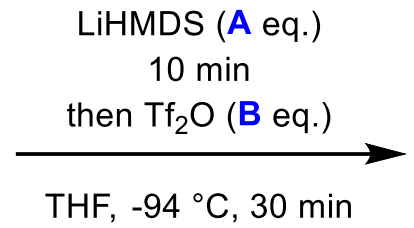

A, B (eq.)<smiles>Cc1cc(C)c(C(=O)N2C=CCCC2)c(C)c1</smiles>

2a

\begin{tabular}{ccc}
\hline Entry & A, B (eq.) & Yield $\left(\%{ }^{b}\right)$ \\
1 & $7.2,3.6$ & 88 \\
2 & $4.8,2.4$ & 90 \\
3 & $3.6,1.8$ & 72 \\
4 & $2.4,1.2$ & 71 \\
5 & $2.4,2.4$ & 67 \\
6 & $7.2,2.4$ & 82
\end{tabular}

a $1 \mathrm{a}(0.30 \mathrm{mmol})$, THF (1.5 mL), LiHMDS (1 M solution in THF) with slow addition, $10 \mathrm{~min}$, then $\mathrm{Tf}_{2} \mathrm{O}$ with addition over $1 \mathrm{~min} .{ }^{\mathrm{b}} \mathrm{GC}$ yield. 
Table S5. Optimization of the addition order. ${ }^{a}$<smiles>Cc1cc(C)c(C(=O)N2CCCCC2)c(C)c1</smiles>

$1 \mathrm{a}$

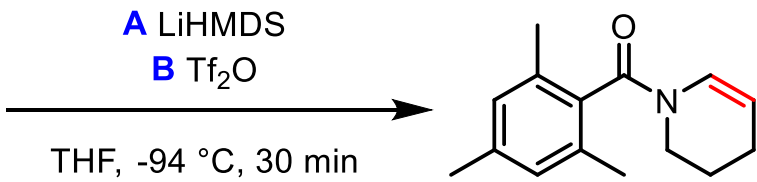

$2 a$

\begin{tabular}{|c|c|c|c|c|}
\hline Entry & $\begin{array}{l}\text { 1st addition } \\
\text { (time) }\end{array}$ & $\begin{array}{l}\text { reaction time } \\
\text { after } 1 \mathrm{st} \text { addtion }\end{array}$ & $\begin{array}{l}\text { 2nd addition } \\
\text { (time) }\end{array}$ & Yield $\left(\%^{b}\right)$ \\
\hline 1 & $B(1 \mathrm{~min})$ & $3 \mathrm{~min}$ & $A(1 \mathrm{~min})$ & 50 \\
\hline 2 & $A(1 \mathrm{~min})$ & $3 \mathrm{~min}$ & $B(1 \mathrm{~min})$ & 88 \\
\hline 3 & $A(1 \mathrm{~min})$ & - & $B(1 \mathrm{~min})$ & 64 \\
\hline 4 & $A(1 \mathrm{~min})$ & $10 \min$ & $B(1 \mathrm{~min})$ & 90 \\
\hline 5 & $A(1 \mathrm{~min})$ & $3 \mathrm{~min}$ & B (all at once) & 68 \\
\hline 6 & $A(1 \mathrm{~min})$ & $3 \mathrm{~min}$ & $B(3 \mathrm{~min})$ & 80 \\
\hline 7 & $A(1 \mathrm{~min})$ & $3 \mathrm{~min}$ & $B(1 \mathrm{~min})^{\mathrm{C}}$ & 83 \\
\hline 8 & premixed $\mathrm{A}+\mathrm{B}^{\mathrm{d}}$ & $3 \mathrm{~min}$ & $1 \mathrm{a}(1 \mathrm{~min})$ & $22^{e}$ \\
\hline
\end{tabular}

a $1 \mathrm{a}(0.30 \mathrm{mmol})$, LiHMDS (0.96 mmol, $1 \mathrm{M}$ solution in THF), $10 \mathrm{~min}, \mathrm{Tf}_{2} \mathrm{O}(0.48 \mathrm{mmol}), 1.5 \mathrm{~mL}$ THF. ${ }^{\mathrm{b}} \mathrm{GC}$ yield. ${ }^{\mathrm{c}}$ equivalents of $\mathrm{A}$ have been halved, addition of $2^{\text {nd }}$ portion of $A 10 \mathrm{~min}$ after the addition of $B$. ${ }^{\mathrm{d}} B$ was added to $A$ over $1 \mathrm{~min}, 10 \mathrm{~min}$, slow addition of $1 \mathrm{a}$. ${ }^{\mathrm{e}}$ isolated yield, major amounts of decomposition were observed. 
Table S6. Optimization of the concentration. ${ }^{\mathrm{a}}$<smiles>Cc1cc(C)c(C(=O)N2CCCCC2)c(C)c1</smiles>

1a

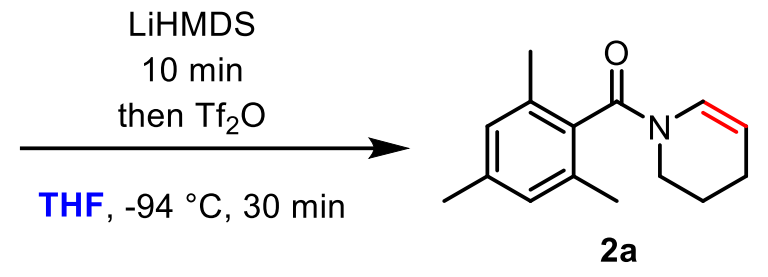

Total solvent volume in $\mathrm{mL}$ $(\text { Molarity })^{\mathrm{b}}$ $1.5(0.2 \mathrm{M}) \quad 40$

$2.25(0.13 \mathrm{M}) \quad 83$

$3(0.1 \mathrm{M}) \quad 90$

$4 \quad 3.75(0.08 \mathrm{M}) \quad 79$

$5 \quad 6(0.05 \mathrm{M}) \quad 22$

a 1 a $(0.30 \mathrm{mmol})$, LiHMDS $\left(0.96 \mathrm{mmol}, 1 \mathrm{M}\right.$ solution in THF) with slow addition, $10 \mathrm{~min}, \mathrm{Tf}_{2} \mathrm{O}(0.48 \mathrm{mmol})$ with addition over $1 \mathrm{~min}$. ${ }^{\mathrm{b}}$ total solvent volume including $1.5 \mathrm{~mL}$ of the LiHMDS solution. ${ }^{\mathrm{c}} \mathrm{GC}$ yield.

Table S7. Optimization of the solvent. ${ }^{\mathrm{a}}$<smiles>Cc1cc(C)c(C(=O)N2CCCCC2)c(C)c1</smiles>

1a

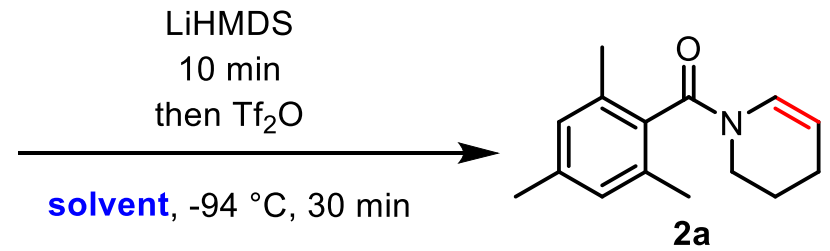

\begin{tabular}{|c|c|c|}
\hline Entry & Solvent $(m L)^{b}$ & Yield $(\%)^{\mathrm{c}}$ \\
\hline 1 & THF & 90 \\
\hline 2 & $\mathrm{Et}_{2} \mathrm{O}$ & 70 \\
\hline 3 & 2-MeTHF & 36 \\
\hline 4 & 2-MeTHF & 50 \\
\hline 5 & toluene & 27 \\
\hline 6 & $\mathrm{DCM}$ & 53 \\
\hline 7 & $\mathrm{Et}_{2} \mathrm{O}+\mathrm{THF}$ & $94\left(89^{\mathrm{e}}\right)$ \\
\hline 8 & 2-MeTHF + THF & 92 \\
\hline 9 & toluene $+\mathrm{THF}$ & 85 \\
\hline
\end{tabular}

a $10.30 \mathrm{mmol})$, solvent $(1.5 \mathrm{~mL})$, LiHMDS $(0.96 \mathrm{mmol}, 1 \mathrm{M}$ solution in the corresponding solvent) with slow addition, $10 \mathrm{~min}$, then Tf $2 \mathrm{O}$ $(0.48 \mathrm{mmol})$ with addition over $1 \mathrm{~min} .{ }^{\mathrm{b}}$ total solvent volume $3 \mathrm{~mL}, 2^{\text {nd }}$ named solvent from the LiHMDS solution. ${ }^{\mathrm{c}} \mathrm{GC}$ yield. ${ }^{\mathrm{d}}-116^{\circ} \mathrm{C}$ instead of $-94{ }^{\circ} \mathrm{C}$. ${ }^{\mathrm{e}}$ isolated yield. 


\section{Experimental}

\subsection{General Procedures}

\subsubsection{General Procedure 1A (GP1A): Synthesis of Amides from Acyl Chlorides}

(1a-o except for $\mathbf{1 c}, \mathbf{3 d - I , 3 n - 0 )}$

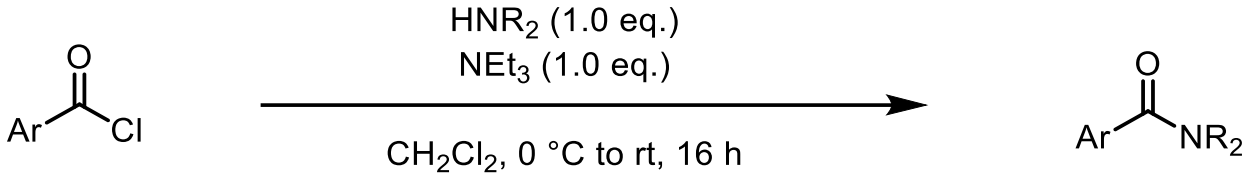

All reactions were run on a $5 \mathrm{mmol}$ scale if not indicated otherwise.

The corresponding acyl chloride (1.0 eq.) was dissolved in $\mathrm{CH}_{2} \mathrm{Cl}_{2}(0.6 \mathrm{M})$ and the resulting mixture was cooled to $0{ }^{\circ} \mathrm{C}$. Then, the amine (1.0 eq.) was added dropwise followed by the slow addition of triethylamine (1.0 eq.). After completion of addition, the ice bath was removed and the reaction was stirred for $16 \mathrm{~h}$. The solution was transferred into a separatory funnel and sequentially washed with $\mathrm{H}_{2} \mathrm{O}, 1 \mathrm{M} \mathrm{NaOH}, 1 \mathrm{~m} \mathrm{HCl}$ and brine. The organic phase was dried over anhydrous $\mathrm{MgSO}_{4}$ and the filtrate was concentrated under reduced pressure giving rise to the desired amide. Depending on the purity of the obtained material as analysed by ${ }^{1} \mathrm{H} N M R$, additional flash column chromatography was performed $\left(\mathrm{SiO}_{2}\right.$, heptane/EtOAc).

\subsubsection{General Procedure 1B (GP1B): Synthesis of Amides from Acyl Chlorides}

(1c, 3c)<smiles>O=C([Al+2])Cl</smiles>

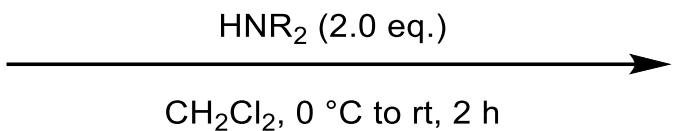<smiles>[R20]C(=O)[AlH2]</smiles>

All reactions were run on a $2 \mathrm{mmol}$ scale if not indicated otherwise.

The corresponding acyl chloride (1.0 eq.) was dissolved in $\mathrm{CH}_{2} \mathrm{Cl}_{2}(0.1 \mathrm{M})$ and the resulting mixture was cooled to $0{ }^{\circ} \mathrm{C}$. Then, the amine ( 2.0 eq.) was added dropwise. After completion of addition, the ice bath was removed and the reaction was stirred for $2 \mathrm{~h}$. The solution was transferred into a separatory funnel and sequentially washed with $1 \mathrm{M} \mathrm{HCl}$ and brine. The organic phase was dried over anhydrous $\mathrm{MgSO}_{4}$ and the filtrate was concentrated under reduced pressure and the resulting crude material was purified via flash column chromatography $\left(\mathrm{SiO}_{2}\right.$, heptane/EtOAC).

\subsubsection{General Procedure 2A (GP2A): Synthesis of Amides from Carboxylic Acids} $(3 m, 3 p, 3 q, 3 r)$<smiles>O=C(O)[Al+2]</smiles>

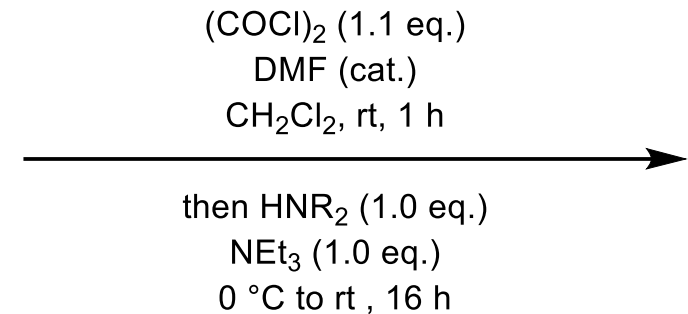<smiles>[R2]NC(=O)[AlH2]</smiles>

All reactions were run on a $5 \mathrm{mmol}$ scale if not indicated otherwise.

The corresponding carboxylic acid (1.0 eq.) was dissolved in $\mathrm{CH}_{2} \mathrm{Cl}_{2}(0.6 \mathrm{M})$ and a few drops of dimethylformamide were added. Oxalyl chloride (1.1 eq.) was added dropwise whereby a strong gas evolution was observed. After $1 \mathrm{~h}$, the amine (1.0 eq.) was slowly added, followed by the addition of 
triethylamine (1.0 eq.). The reaction was stirred for $16 \mathrm{~h}$. The solution was transferred into a separatory funnel and sequentially washed with $\mathrm{H}_{2} \mathrm{O}, 1 \mathrm{M} \mathrm{NaOH}, 1 \mathrm{M} \mathrm{HCl}$ and finally brine. The organic phase was dried over anhydrous $\mathrm{MgSO}_{4}$ and the filtrate was concentrated under reduced pressure giving rise to the desired amide. Depending on the purity of the obtained material as analysed by ${ }^{1} \mathrm{H} N \mathrm{NR}$, additional flash column chromatography was performed $\left(\mathrm{SiO}_{2}\right.$, heptane/EtOAc).

\subsubsection{General Procedure 2B (GP2B): Synthesis of Amides from Carboxylic Acids} $(3 a, 3 b, 3 u)$

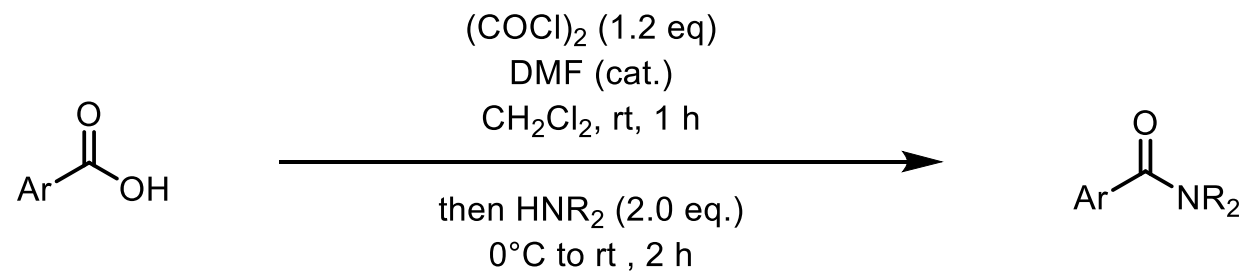

The corresponding carboxylic acid (1.0 eq.) was dissolved in $\mathrm{CH}_{2} \mathrm{Cl}_{2}(0.2 \mathrm{M})$ and a few drops of DMF were added. Oxalyl chloride (1.2 eq.) was added dropwise whereby a strong gas evolution was observed. After $1 \mathrm{~h}$, the amine (2.0 eq.) was slowly added. The reaction was stirred for $2 \mathrm{~h}$. The solution was transferred into a separatory funnel and sequentially washed with $1 \mathrm{~m} \mathrm{HCl}$ and brine. The organic phase was dried over anhydrous $\mathrm{MgSO}_{4}$ and the filtrate was concentrated under reduced pressure giving rise to the desired amide. The crude material was additionally purified via flash column chromatography $\left(\mathrm{SiO}_{2}\right.$, heptanes/EtOAc).

\subsubsection{General Procedure 3 (GP3): Synthesis of Enamides}

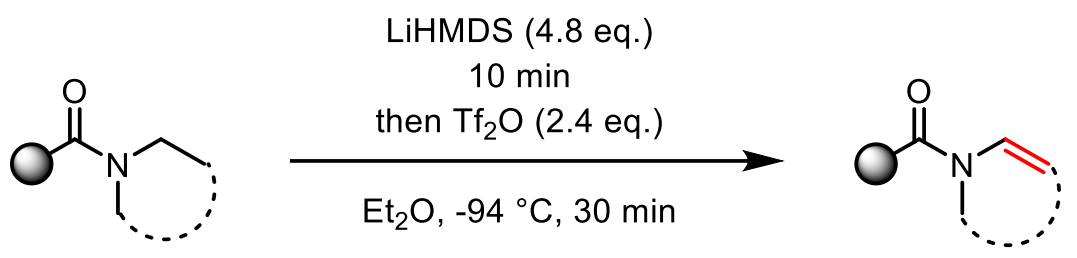

A flame-dried Schlenk flask was loaded with the corresponding amide $(0.30 \mathrm{mmol}, 1.00$ eq. $)$ which was dissolved in anhydrous $\mathrm{Et}_{2} \mathrm{O}(1.5 \mathrm{~mL})$. The solution was cooled down to $-94{ }^{\circ} \mathrm{C}$ (dry ice/acetone mixture). Then LiHMDS ( $1 \mathrm{~m}$ in THF, $1.44 \mathrm{~mL}, 1.44 \mathrm{mmol}, 4.80$ eq.) was added slowly (ca. $20 \mathrm{~s}$ ). After the addition, the resulting mixture was stirred for $10 \mathrm{~min}$. Following $\mathrm{Tf}_{2} \mathrm{O}(121 \mu \mathrm{L}, 0.72 \mathrm{mmol}, 2.40$ eq.) was added dropwise over 1 min with vigorous stirring (very important). The reaction was stirred for $30 \mathrm{~min}$ before remaining reagents were quenched by the addition of a saturated solution of $\mathrm{NH}_{4} \mathrm{Cl}$ $(10 \mathrm{~mL})$. The solution was transferred into a separatory funnel and extracted with $\mathrm{CH}_{2} \mathrm{Cl}_{2}(3 \times 10 \mathrm{~mL})$. The combined organic phases were dried over anhydrous $\mathrm{MgSO}_{4}$ and the solvent was removed under reduced pressure. The crude material was purified by column chromatography $\left(\mathrm{SiO}_{2}\right.$, heptanes/EtOAc). Importantly, a noticeable instability of enamides on silica gel has been observed. Therefore, the silica gel was treated with $\mathrm{NEt}_{3} / \mathrm{Et}_{2} \mathrm{O}(1: 9)$, followed by drying in the air prior to use in the flash column chromatography.

\section{Large scale:}

For a large scale $(5.5$ or $15.2 \mathrm{mmol})$ the addition time of $\mathrm{Tf}_{2} \mathrm{O}$ was extended $(0.28$ or $0.76 \mathrm{mmol} / \mathrm{min})$ and also the time before the addition of $\mathrm{Tf}_{2} \mathrm{O}$ was prolonged ( $20 \mathrm{~min}$, both cases) to ensure a satisfying cooling. 


\subsection{Characterization of Starting Materials}

\section{Mesityl(piperidin-1-yl)methanone (1a)}<smiles>Cc1cc(C)c(C(=O)N2CCCCC2)c(C)c1</smiles>

Following the GP1 using 2,4,6-trimethylbenzoyl chloride and piperidine afforded the desired amide $(1.15 \mathrm{~g}, 99 \%)$ as yellowish oil. ${ }^{1} \mathbf{H} \mathbf{~ N M R ~}\left(400 \mathrm{MHz}, \mathrm{CDCl}_{3}\right): \delta$ $6.83(\mathrm{~s}, 2 \mathrm{H}), 3.76(\mathrm{app} \mathrm{s}, 2 \mathrm{H}), 3.16-3.11(\mathrm{~m}, 2 \mathrm{H}), 2.26(\mathrm{~s}, 3 \mathrm{H}), 2.21(\mathrm{~s}, 6 \mathrm{H}), 1.67$ $-1.64(\mathrm{~m}, 4 \mathrm{H}), 1.46(\mathrm{app} \mathrm{s}, 2 \mathrm{H}) \mathrm{ppm}$. Spectral data was found in agreement with the literature. ${ }^{[2]}$

\section{Mesityl(pyrrolidin-1-yl)methanone (1b)}<smiles>Cc1cc(C)c(C(=O)N2CCCC2)c(C)c1</smiles>

Following the GP1A using 2,4,6-trimethylbenzoyl chloride and pyrrolidine afforded the desired amide $(1.06 \mathrm{~g}, 98 \%)$ as yellowish oil. ${ }^{1} \mathbf{H}$ NMR $(400 \mathbf{~ M H z}$, $\left.\mathrm{CDCl}_{3}\right): 6.83(\mathrm{~s}, 2 \mathrm{H}), 3.67(\mathrm{t}, J=6.9 \mathrm{~Hz}, 2 \mathrm{H}), 3.03(\mathrm{t}, J=6.7 \mathrm{~Hz}, 2 \mathrm{H}), 2.26(\mathrm{~s}, 3 \mathrm{H})$, $2.21(\mathrm{~s}, 6 \mathrm{H}), 2.00-1.91(\mathrm{~m}, 2 \mathrm{H}), 1.89-1.81(\mathrm{~m}, 2 \mathrm{H}) \mathrm{ppm}$. Spectral data was found in agreement with the literature. ${ }^{[3]}$

\section{Azepan-1-yl(mesityl)methanone (1c)}<smiles>Cc1cc(C)c(C(=O)N2CCCCCC2)c(C)c1</smiles>

Following the GP1B using 2,4,6-trimethylbenzoyl chloride and pyrrolidine afforded the desired amide (476 mg, 97\%) as yellowish oil. ${ }^{1} \mathrm{H}$ NMR $(600 \mathrm{MHz}$, $\left.\mathrm{CDCl}_{3}\right): \delta 6.83(\mathrm{~s}, 2 \mathrm{H}), 3.74-3.68(\mathrm{~m}, 2 \mathrm{H}), 3.20-3.15(\mathrm{~m}, 2 \mathrm{H}), 2.26(\mathrm{~s}, 3 \mathrm{H})$, $2.22(\mathrm{~s}, 6 \mathrm{H}), 1.87-1.78(\mathrm{~m}, 2 \mathrm{H}), 1.68-1.61(\mathrm{~m}, 2 \mathrm{H}), 1.60-1.54(\mathrm{~m}, 4 \mathrm{H}) \mathrm{ppm}$. ${ }^{13} \mathrm{C}$ NMR (101 MHz, $\left.\mathrm{CDCl}_{3}\right): \delta 171.4,137.8,134.5,133.4,128.4,48.6,44.7$, 29.0, 28.4, 28.1, 26.9, 21.2, 19.2 ppm. IR (neat) $\mathbf{v}_{\text {max }}$ : 2923, 2856, 1628, 1438, 1416, 1377, 1305, 1278, $852,764,750$. $\left.\left.\mathrm{HRMS}_{(\mathrm{ESI}}\right)^{+}\right)$exact mass calculated for $[\mathrm{M}+\mathrm{Na}]^{+}\left(\mathrm{C}_{16} \mathrm{H}_{23} \mathrm{NNaO}^{+}\right)$requires $\mathrm{m} / \mathrm{z} 268.1672$, found $\mathrm{m} / \mathrm{z} 268.1673$.

\section{Mesityl(1,4-dioxa-8-azaspiro[4.5]decan-8-yl)methanone (1d)}<smiles>Cc1cc(C)c(C(=O)N2CCC3(CC2)OCCO3)c(C)c1</smiles>

Following the GP1A using 2,4,6-trimethylbenzoyl chloride and 1,4-dioxa-8azaspiro[4.5]decane afforded the desired amide $(1.3 \mathrm{~g}, 90 \%)$ as white solid. ${ }^{1} \mathrm{H}$ NMR $\left(400 \mathrm{MHz}, \mathrm{CDCl}_{3}\right): \delta 6.83(\mathrm{~s}, 2 \mathrm{H}), 4.03-3.87(\mathrm{~m}, 6 \mathrm{H}), 3.30-3.25$ $(\mathrm{m}, 2 \mathrm{H}), 2.26(\mathrm{~s}, 3 \mathrm{H}), 2.20(\mathrm{~s}, 6 \mathrm{H}), 1.81-1.76(\mathrm{~m}, 2 \mathrm{H}), 1.61-1.58(\mathrm{~m}, 2 \mathrm{H})$ ppm. ${ }^{13} \mathrm{C}$ NMR (101 MHz, $\left.\mathrm{CDCl}_{3}\right): \delta$ 169.9, 138.1, 133.7, 133.5, 128.4, 107.1,

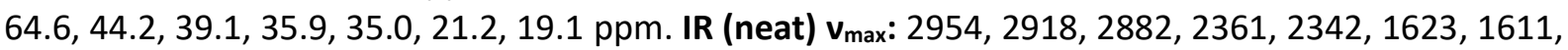
1466, 1435, 1356, 1245, 1183, 1146, 1114, 1077, 1029, 944, 913, 863. HRMS (ESI ${ }^{+}$: exact mass calculated for $[\mathrm{M}+\mathrm{H}]^{+}\left(\mathrm{C}_{17} \mathrm{H}_{24} \mathrm{NO}_{3}{ }^{+}\right)$requires $\mathrm{m} / \mathrm{z} 290.1751$ found $\mathrm{m} / \mathrm{z} 290.1738$.

\section{Mesityl(octahydro-2H-isoindol-2-yl)methanone (1e)}

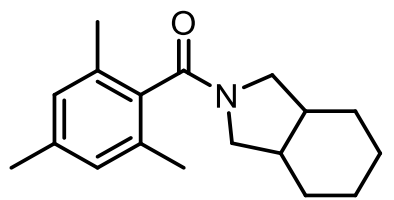

Following the GP1A using 2,4,6-trimethylbenzoyl chloride and octahydro$1 \mathrm{H}$-isoindole afforded the desired amide $(1.19 \mathrm{~g}, 88 \%)$ as colorless oil. ${ }^{1} \mathbf{H}$ NMR (400 MHz, $\left.\mathrm{CDCl}_{3}\right): \delta 6.84(\mathrm{~s}, 2 \mathrm{H}), 3.63-3.56(\mathrm{~m}, 2 \mathrm{H}), 3.09-3.03(\mathrm{~m}$, $1 \mathrm{H}), 2.91-2.85(\mathrm{~m}, 1 \mathrm{H}), 2.33-2.16(\mathrm{~m}, 11 \mathrm{H}), 1.69-1.32(\mathrm{~m}, 8 \mathrm{H}) \mathrm{ppm} .{ }^{13} \mathrm{C}$ NMR (101 MHz, CDCl $)_{3}: \delta$ 170.9, 138.0, 135.4, 133.2, 133.0, 129.0, 128.4, 51.5, 49.0, 37.5, 36.5, 26.1, 25.8, 22.9, 22.7, 21.3, 19.2, 19.0 ppm. IR (neat) $\boldsymbol{v}_{\text {max }}$ : 2923, 2874, 2855, 1624, 1439, 1409, 1378, 851, 732. HRMS (ESI $\left.{ }^{+}\right)$: exact mass calculated for $[\mathrm{M}+\mathrm{H}]^{+}\left(\mathrm{C}_{18} \mathrm{H}_{26} \mathrm{NO}^{+}\right)$requires $272.2009 \mathrm{~m} / \mathrm{z}$ found $272.1996 \mathrm{~m} / \mathrm{z}$. 
$\mathrm{C}_{\mathrm{O}}^{\mathrm{N}}$

Following the GP1B using 2,4,6-trimethylbenzoyl chloride and morpholine afforded the desired amide (412 mg, 35\%) as colorless oil. $\left.{ }^{1} \mathrm{H} \mathrm{NMR} \mathrm{(400} \mathrm{MHz,} \mathrm{CDCl}_{3}\right): \delta 6.85$ $(\mathrm{s}, 2 \mathrm{H}), 3.86-3.81(\mathrm{~m}, 2 \mathrm{H}), 3.79-3.72(\mathrm{~m}, 2 \mathrm{H}), 3.59-3.54(\mathrm{~m}, 2 \mathrm{H}), 3.21-3.15(\mathrm{~m}$, 2H), $2.27(\mathrm{~s}, 3 \mathrm{H}), 2.22(\mathrm{~s}, 6 \mathrm{H}) \mathrm{ppm} .{ }^{13} \mathrm{C}$ NMR $\left(101 \mathrm{MHz}, \mathrm{CDCl}_{3}\right): \delta 170.1,138.4,133.7$, $132.9,128.5,67.2,67.1,46.6,41.6,21.2,19.2$ ppm. IR (neat) $v_{\text {max }}$ :2916, 2854, 1634, 1456, 1436, 1277, 1255, 1178, 1114, 1010, 841, 751. HRMS (ESI'): exact mass calculated for $[\mathrm{M}+\mathrm{Na}]^{+}\left(\mathrm{C}_{14} \mathrm{H}_{19} \mathrm{NNaO}_{2}^{+}\right)$requires $\mathrm{m} / \mathrm{z} 256.1308$, found $\mathrm{m} / \mathrm{z} 256.1308$.

\section{Ethyl 4-(2,4,6-trimethylbenzoyl)piperazine-1-carboxylate (1g)}<smiles>CCON1CCN(C(=O)c2c(C)cc(C)cc2C)CC1</smiles>

Following the GP1A using 2,4,6-trimethylbenzoyl chloride and ethyl piperazine-1-carboxylate afforded the desired amide $(1.47 \mathrm{~g}, 97 \%)$ as white solid. ${ }^{1} \mathrm{H}$ NMR (400 MHz, $\left.\mathrm{CDCl}_{3}\right): \delta 6.85(\mathrm{~s}, 2 \mathrm{H}), 4.15$ (q, $J=7.0 \mathrm{~Hz}$, $2 \mathrm{H}), 3.81(\mathrm{t}, J=5.1 \mathrm{~Hz}, 2 \mathrm{H}), 3.58-3.53(\mathrm{~m}, 2 \mathrm{H}), 3.37(\mathrm{t}, J=4.8 \mathrm{~Hz}, 2 \mathrm{H})$, $3.17(\mathrm{t}, J=4.4 \mathrm{~Hz}, 2 \mathrm{H}), 2.27(\mathrm{~s}, 3 \mathrm{H}), 2.20(\mathrm{~s}, 6 \mathrm{H}), 1.26(\mathrm{t}, J=6.5 \mathrm{~Hz}, 3 \mathrm{H})$ ppm. ${ }^{13} \mathrm{C}$ NMR (101 MHz, CDCl $): \delta 170.2,155.5,138.5,133.6,132.9,128.5,61.9,45.9,41.0,21.2,19.1$, 14.7 ppm. IR (neat) $\mathbf{v}_{\text {max }}: 2984,2917,2853,1697,1638,1422,1382,1227,1178,1114,1004,990,852$, 766. HRMS (ESI $\left.{ }^{+}\right)$: exact mass calculated for $[\mathrm{M}+\mathrm{H}]^{+}\left(\mathrm{C}_{17} \mathrm{H}_{25} \mathrm{~N}_{2} \mathrm{O}_{3}{ }^{+}\right)$requires $305.1860 \mathrm{~m} / \mathrm{z}$ found $305.1844 \mathrm{~m} / \mathrm{z}$.

\section{$\mathrm{N}, \mathrm{N}$-Diethyl-2,4,6-trimethylbenzamide (1h)}

Following the GP1A using 2,4,6-trimethylbenzoyl chloride and diethylamine afforded the desired<smiles>CCN(CC)C(=O)c1c(C)cc(C)cc1C</smiles>
amide $(1.06 \mathrm{~g}, 97 \%)$ as white solid. ${ }^{1} \mathbf{H}$ NMR $\left(400 \mathrm{MHz}, \mathrm{CDCl}_{3}\right): \delta 6.83(\mathrm{~s}, 2 \mathrm{H})$, $3.60(\mathrm{q}, J=6.9 \mathrm{~Hz}, 2 \mathrm{H}), 3.11(\mathrm{q}, J=7.0 \mathrm{~Hz}, 2 \mathrm{H}), 2.27(\mathrm{~s}, 3 \mathrm{H}), 2.20(\mathrm{~s}, 6 \mathrm{H}), 1.26(\mathrm{t}$, $J=7.0 \mathrm{~Hz}, 3 \mathrm{H}), 1.02(\mathrm{t}, J=7.0 \mathrm{~Hz}, 3 \mathrm{H}) \mathrm{ppm}$. Spectral data was found in agreement with the literature. ${ }^{[4]}$

\section{2,4,6-Trimethyl-N,N-dipropylbenzamide (1i)}<smiles>CCCN(CCC)C(=O)c1c(C)cc(C)cc1C</smiles>

Following the GP1A using 2,4,6-trimethylbenzoyl chloride and dipropylamine afforded the desired amide $(1.17 \mathrm{~g}, 95 \%)$ as white solid. ${ }^{1} \mathbf{H} \mathbf{~ N M R}(400 \mathrm{MHz}$, $\left.\mathrm{CDCl}_{3}\right): \delta 6.82(\mathrm{~s}, 2 \mathrm{H}), 3.52-3.44(\mathrm{~m}, 2 \mathrm{H}), 3.05-2.93(\mathrm{~m}, 2 \mathrm{H}), 2.20(\mathrm{~s}, 6 \mathrm{H})$, $1.78-1.64(\mathrm{~m}, 2 \mathrm{H}), 1.49-1.37(\mathrm{~m}, 2 \mathrm{H}), 0.98(\mathrm{t}, J=7.4 \mathrm{~Hz}, 3 \mathrm{H}), 0.73(\mathrm{t}, J=7.4$ $\mathrm{Hz}, 3 \mathrm{H})$ ppm. ${ }^{13} \mathrm{C}$ NMR (101 MHz, $\left.\mathrm{CDCl}_{3}\right): \delta 171.2,137.7,134.3,133.5,128.4$, 50.0, 46.1, 21.7, 21.2, 20.8, 19.2, 11.8, 11.5 ppm. IR (neat) $\mathbf{v}_{\text {max }}$ :2969, 2932, 2874, 1618, 1464, 1416, 1378, 1309, 1255, 1091, 896, 855, 755, 595. HRMS (ESI ${ }^{+}$: exact mass calculated for $[\mathrm{M}+\mathrm{Na}]^{+}$ $\left(\mathrm{C}_{16} \mathrm{H}_{25} \mathrm{NNaO}^{+}\right)$requires $\mathrm{m} / \mathrm{z} 270.1828$ found $\mathrm{m} / \mathrm{z} 270.1830$.

\section{N,N-Dibutyl-2,4,6-trimethylbenzamide (1j)}

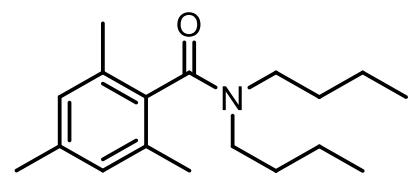

Following the GP1A using 2,4,6-trimethylbenzoyl chloride and dibutylamine afforded the desired amide $(1.12 \mathrm{~g}, 82 \%)$ as colorless liquid. ${ }^{1} \mathrm{H}$ NMR $\left(400 \mathrm{MHz} \mathrm{CDCl}_{3}\right): \delta 6.81(\mathrm{~s}, 2 \mathrm{H}), 3.53-3.48(\mathrm{~m}, 2 \mathrm{H}), 3.03-2.99$ $(\mathrm{m}, 2 \mathrm{H}), 2.26(\mathrm{~s}, 3 \mathrm{H}), 2.19(\mathrm{~s}, 6 \mathrm{H}), 1.71-1.59(\mathrm{~m}, 2 \mathrm{H}), 1.44-1.34(\mathrm{~m}, 4 \mathrm{H})$, $1.16-1.07(\mathrm{~m}, 2 \mathrm{H}), 0.98(\mathrm{t}, J=7.3 \mathrm{~Hz}, 3 \mathrm{H}), 0.77(\mathrm{t}, J=7.3 \mathrm{~Hz}, 3 \mathrm{H})$ ppm. ${ }^{13} \mathrm{C} \mathrm{NMR}\left(101 \mathrm{MHz}, \mathrm{CDCl}_{3}\right): \delta$ 171.1, 137.7, 134.3, 133.5, 128.3, 48.0, 44.1, 30.7, 29.6, 21.2, 20.7, 20.1, 19.2, 14.1, 13.8 ppm. IR (neat) $\mathbf{V}_{\text {max: }}$ 2957, 2930, 2871, 1628, 1612, 1457, 1417, 1377, 1295, 1259, 1234, 1090, 850, 751, 596. HRMS (ESI ${ }^{+}$): exact mass calculated for $[\mathrm{M}+\mathrm{H}]^{+}\left(\mathrm{C}_{18} \mathrm{H}_{30} \mathrm{NO}^{+}\right)$requires $\mathrm{m} / \mathrm{z} 276.2327$ found $\mathrm{m} / \mathrm{z} 276.2331$. 
<smiles>Cc1cc(C)c(C(=O)N2CCCCC2Cc2ccccc2)c(C)c1</smiles>

Following the GP1A (1.37 mmol scale) using 2,4,6-trimethylbenzoyl chloride and 2-benzylpiperidine afforded the desired amide $(277 \mathrm{mg}$, $63 \%)$ as colorless oil. Via NMR spectroscopy rotameric effects were detected with a rotameric ratio of 2.9:1. Both rotamers are described together: ${ }^{1} \mathbf{H}$ NMR $\left(600 \mathrm{MHz}, \mathrm{CDCl}_{3}\right): \delta 7.40-7.29(\mathrm{~m}, 2.8 \mathrm{H}), 7.22-7.13$ $(\mathrm{m}, 1.2 \mathrm{H}), 6.94(\mathrm{~s}, 0.2 \mathrm{H}), 6.85-6.83(\mathrm{~m}, 0.8 \mathrm{H}), 6.76(\mathrm{~s}, 1 \mathrm{H}), 5.35(\mathrm{dd}, J=$ 13.7, 7.3 Hz, 0.7H), $4.88(\mathrm{~d}, J=13.3 \mathrm{~Hz}, 0.3 \mathrm{H}), 3.70-3.64(\mathrm{~m}, 0.3 \mathrm{H}), 3.24(\mathrm{~d}, J=11.5 \mathrm{~Hz}, 0.7 \mathrm{H}), 3.11(\mathrm{t}$, $J=12.8 \mathrm{~Hz}, 1 \mathrm{H}), 3.03-2.99(\mathrm{~m}, J=7.6 \mathrm{~Hz}, 1.4 \mathrm{H}), 2.96-2.89(\mathrm{~m}, 0.3 \mathrm{H}), 2.66-2.62(\mathrm{~m}, J=13.1,2.7 \mathrm{~Hz}$, $0.3 \mathrm{H}), 2.32-2.24(\mathrm{~m}, 6.4 \mathrm{H}), 2.17(\mathrm{~s}, 0.6 \mathrm{H}), 1.87(\mathrm{~s}, 2 \mathrm{H}), 1.82-1.34(\mathrm{~m}, 6 \mathrm{H}) \mathrm{ppm} .{ }^{13} \mathrm{C}$ NMR (151 MHz, $\left.\mathrm{CDCl}_{3}\right): \delta 169.9,169.8,138.3(2 \mathrm{C}), 137.8,137.5,134.0,133.8,133.5,133.4,133.3,129.4,129.0,128.5$, 128.4, 128.3 128.2, 128.0, 126.4, 126.4, 56.2, 48.6, 42.0, 37.0 (2C), 35.6, 30.9, 26.4, 26.2, 25.6, 21.1, $21.0,19.5,19.2,19.1,19.0,18.9,18.4$ ppm. IR (neat) $\mathbf{v}_{\text {max }}: 2934,2856,1623,1436,1416,1287,1032$,

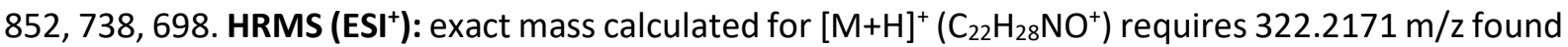
$322.2181 \mathrm{~m} / \mathrm{z}$.

\section{Mesityl(2-methylpiperidin-1-yl)methanone (1l)}<smiles>Cc1cc(C)c(C(=O)N2CCCCC2C)c(C)c1</smiles>

Following the GP1A using 2,4,6-trimethylbenzoyl chloride and 2methylpiperidine afforded the desired amide $(1.02 \mathrm{~g}, 83 \%)$ as colorless oil. The desired amide was obtained in a rotameric mixture with a ratio of around 2:1. Major isomer: ${ }^{1} \mathrm{H} \mathrm{NMR}\left(600 \mathrm{MHz}, \mathrm{CDCl}_{3}\right): \delta 6.84(\mathrm{~s}, 2 \mathrm{H}), 5.20-5.14(\mathrm{~m}, 1 \mathrm{H}), 3.19$ (dd, J = 13.5, $2.5 \mathrm{~Hz}, 1 \mathrm{H}), 3.04(\mathrm{td}, \mathrm{J}=13.3,3.0 \mathrm{~Hz}, 1 \mathrm{H}), 2.26(\mathrm{~s}, 6 \mathrm{H}), 2.14(\mathrm{~s}, 3 \mathrm{H}), 1.75-1.46(\mathrm{~m}, 6 \mathrm{H})$, $1.28(\mathrm{~d}, \mathrm{~J}=7.0 \mathrm{~Hz}, 3 \mathrm{H}) \mathrm{ppm}$. Minor isomer: ${ }^{1} \mathrm{H}$ NMR $\left(600 \mathrm{MHz}, \mathrm{CDCl}_{3}\right): \delta 6.81(\mathrm{~s}, 2 \mathrm{H}), 4.80-4.69(\mathrm{~m}$, $1 \mathrm{H}), 3.76-3.67(\mathrm{~m}, 1 \mathrm{H}), 2.86(\mathrm{td}, \mathrm{J}=13.3,3.0 \mathrm{~Hz}, 1 \mathrm{H}), 2.27(\mathrm{~s}, 6 \mathrm{H}), 2.23(\mathrm{~s}, 3 \mathrm{H}), 1.75-1.46(\mathrm{~m}, 6 \mathrm{H})$, $1.14(\mathrm{~d}, \mathrm{~J}=6.9 \mathrm{~Hz}, 3 \mathrm{H}) \mathrm{ppm}$. The ${ }^{13} \mathrm{C}$ carbon signals of both rotamers could not be clearly differentiated. ${ }^{13} \mathrm{C}$ NMR (151 MHz, CDCl $): \delta 169.8,169.7,137.7$ (2C), 134.3, 134.2, $133.6(2 \mathrm{C}), 133.5,133.4,128.6$, $128.4,128.2$ (2C), 50.0, 43.4, 41.6, 36.2, 31.5, 30.1, 26.8, 26.0, 21.2, 19.5, 19.4, 19.2, 19.1 (2C), 18.8, $17.0,15.7 \mathrm{ppm}$. IR (neat) $\mathbf{v}_{\text {max }}: 2969,2934,2856,1624,1611,1437,1416,1273,1266,1107,1032,850$, 733. HRMS (ESI $\left.{ }^{+}\right)$: exact mass calculated for $[\mathrm{M}+\mathrm{H}]^{+}\left(\mathrm{C}_{16} \mathrm{H}_{24} \mathrm{NO}^{+}\right)$requires $246.1858 \mathrm{~m} / \mathrm{z}$ found 246.1842 $\mathrm{m} / \mathrm{z}$.

\section{MesityI(2-phenylpyrrolidin-1-yl)methanone (1m)}

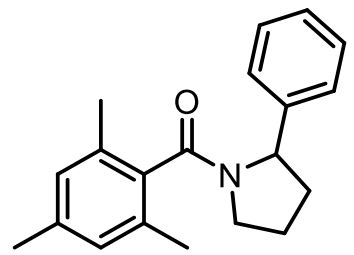

Following the GP1A using 2,4,6-trimethylbenzoyl chloride and 2phenylpyrrolidine afforded the desired amide $(1.21 \mathrm{~g}, 83 \%)$ as colorless oil. NMR spectroscopy analysis revealed two isomers in a ratio of around 1:1. Both isomers are described together: ${ }^{1} \mathrm{H}$ NMR $\left(600 \mathrm{MHz}, \mathrm{CDCl}_{3}\right): \delta 7.36-7.33(\mathrm{~m}$, $2 \mathrm{H}), 7.26-7.23(\mathrm{~m}, 0.5 \mathrm{H}), 7.18-7.13(\mathrm{~m}, 1.5 \mathrm{H}), 6.85(\mathrm{~s}, 1 \mathrm{H}), 6.83-6.80(\mathrm{~m}$, $1 \mathrm{H}), 6.80(\mathrm{~s}, 0.5 \mathrm{H}), 6.48(\mathrm{~s}, 0.5 \mathrm{H}), 5.40(\mathrm{dd}, J=8.1,3.4 \mathrm{~Hz}, 0.5 \mathrm{H}), 4.45(\mathrm{dd}, J=$ 7.9, $3.4 \mathrm{~Hz}, 0.5 \mathrm{H}), 4.09-4.04(\mathrm{~m}, 0.5 \mathrm{H}), 3.90-3.83(\mathrm{~m}, 0.5 \mathrm{H}), 3.37-3.32(\mathrm{~m}, 1 \mathrm{H}), 3.25-3.20(\mathrm{~m}$, $0.5 \mathrm{H}), 2.39-2.30(\mathrm{~m}, 1 \mathrm{H}), 2.28(\mathrm{~s}, 3 \mathrm{H}), 2.23(\mathrm{~s}, 3 \mathrm{H}), 2.20(\mathrm{~s}, 1.5 \mathrm{H}), 2.05-1.83(\mathrm{~m}, 3 \mathrm{H}), 1.56(\mathrm{~s}, 1.5 \mathrm{H})$ ppm. ${ }^{13} \mathrm{C}$ NMR (151 MHz, CDCl $): \delta$ 171.3, 170.3, 143.6, 143.4, 138.0, 137.9, 135.2, 134.9, 134.6, 133.5, $133.0,132.2,128.6,128.5,128.4$ (2C), 128.3, 127.8, 127.2, 127.0, 126.3, 126.1, 63.1, 60.40, 48.7, 46.6, 36.0, 34.7, 24.2, 22.8, 21.2 (2C), 19.3, 19.2, 19.1, 18.9 ppm. IR (neat) $\mathbf{v}_{\text {max }}$ : 2971, 2923, 2868, 1620, $1611,1447,1437,1401,845,701$. HRMS $\left._{(\mathbf{E S I}}\right)^{+}$: exact mass calculated for $[\mathrm{M}+\mathrm{H}]^{+}\left(\mathrm{C}_{20} \mathrm{H}_{24} \mathrm{NO}^{+}\right)$requires $294.1858 \mathrm{~m} / \mathrm{z}$ found $\mathrm{m} / \mathrm{z} 294.1866$. 
<smiles>Cc1cc(C)c(C(=O)N2CCC[C@H]3CCCC[C@H]32)c(C)c1</smiles>

Following the GP1A using 2,4,6-trimethylbenzoyl chloride and (4aS,8aR)decahydroquinoline afforded the desired amide $(842 \mathrm{mg}, 59 \%)$ as colorless crystals. ${ }^{1} \mathrm{H}$ NMR $\left(600 \mathrm{MHz}, \mathrm{CDCl}_{3}\right): \delta 6.85-6.78(\mathrm{~m}, 2 \mathrm{H}), 3.67$ (td, $J=11.0$, $3.0 \mathrm{~Hz}, 1 \mathrm{H}), 3.24-3.15(\mathrm{~m}, 1 \mathrm{H}), 3.05(\mathrm{dd}, J=13.5,6.9 \mathrm{~Hz}, 1 \mathrm{H}), 2.51-2.42$ $(\mathrm{m}, 1 \mathrm{H}), 2.24(\mathrm{~d}, J=2.7 \mathrm{~Hz}, 6 \mathrm{H}), 2.16(\mathrm{~s}, 3 \mathrm{H}), 1.84-1.48(\mathrm{~m}, 8 \mathrm{H}), 1.35-1.05(\mathrm{~m}, 4 \mathrm{H}) \mathrm{ppm} .{ }^{13} \mathrm{C}$ NMR (151 MHz, $\mathrm{CDCl}_{3}$ ): $\delta$ 170.6, 137.6, 134.7, 133.7, 133.6, 128.4, 128.1, 60.5, 40.0, 38.3, 33.0, 29.9, 26.4, $25.9,25.4,23.5,21.1,19.2,18.6$ ppm. IR (neat) $v_{\text {max }}$ : 2923, 3856, 620, 1440, 1416, 1360, 1266, 1251, 1170, 1096, 1010, 851, 732, 701, 565. HRMS $\left(\mathrm{ESI}^{+}\right)$: exact mass calculated for $[\mathrm{M}+\mathrm{H}]^{+}\left(\mathrm{C}_{19} \mathrm{H}_{28} \mathrm{NO}^{+}\right)$ requires $\mathrm{m} / \mathrm{z} 286.2165$ found $\mathrm{m} / \mathrm{z} 286.2176$.

\section{$\mathrm{N}$-Cyclohexyl-N-ethyl-2,4,6-trimethylbenzamide (10)}

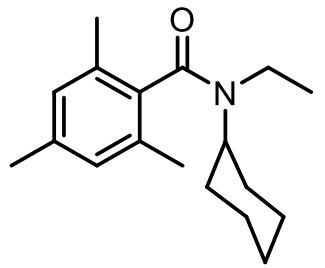

Following the GP1A using 2,4,6-trimethylbenzoyl chloride and $\mathrm{N}$ ethylcyclohexanamine afforded the desired amide $(1.05 \mathrm{~g}, 77 \%)$ as colorless oil. NMR spectroscopy analysis revealed two rotamers in a ratio of $2: 1$. Both rotamers are described together: ${ }^{1} \mathrm{H} \mathbf{N M R}\left(400 \mathrm{MHz}, \mathrm{CDCl}_{3}\right): \delta 6.81(\mathrm{~s}, 2 \mathrm{H}), 4.48$ $(\mathrm{t}, J=12.0 \mathrm{~Hz}, 0.4 \mathrm{H}), 3.49(\mathrm{q}, J=6.8 \mathrm{~Hz}, 1.2 \mathrm{H}), 3.18(\mathrm{t}, J=11.8 \mathrm{~Hz}, 0.6 \mathrm{H}), 3.06$ $(\mathrm{q}, J=7.0 \mathrm{~Hz}, 0.8 \mathrm{H}), 2.27(\mathrm{~s}, 3 \mathrm{H}), 2.21(\mathrm{~s}, 6 \mathrm{H}), 1.95-1.76(\mathrm{~m}, 2 \mathrm{H}), 1.75-1.40(\mathrm{~m}$, $6 \mathrm{H}), 1.31(\mathrm{t}, J=6.9 \mathrm{~Hz}, 2 \mathrm{H}), 1.08-0.85(\mathrm{~m}, 4 \mathrm{H}) \mathrm{ppm} .{ }^{13} \mathrm{C} \mathrm{NMR}\left(151 \mathrm{MHz}, \mathrm{CDCl}_{3}\right): \delta 171.2,170.8,137.7$, $137.4,135.0,134.5,133.5,133.4,128.4,59.0,53.8,39.5,36.2$, 32.3, 31.1, 26.1, 25.9, 25.8, 25.4, 21.2, 19.2 (2C), 16.1, 14.9 ppm. IR (neat) $\mathbf{v}_{\text {max }}$ :2971, 2928, 2855, 1626, 1612, 1466, 1450, 1413, 1369, 1307, $1153,1076,850$. HRMS (ESI $\left.{ }^{+}\right)$: exact mass calculated for $[\mathrm{M}+\mathrm{H}]^{+}\left(\mathrm{C}_{18} \mathrm{H}_{28} \mathrm{NO}^{+}\right)$requires $274.2171 \mathrm{~m} / \mathrm{z}$ found $274.2175 \mathrm{~m} / \mathrm{z}$.

\section{N-Butyl-N-ethyl-2,4,6-trimethylbenzamide (1p)}<smiles>CCCCN(CC)C(=O)c1c(C)cc(C)cc1C</smiles>

Following the GP1A using 2,4,6-trimethylbenzoyl chloride and N-ethylbutan1-amine afforded the desired amide $(1.16 \mathrm{~g}, 94 \%)$ as colorless oil. NMR spectroscopy analysis revealed two rotamers in a ratio of 1.2:1. Both rotamers are described together: ${ }^{1} \mathrm{H}$ NMR $\left.\left(600 \mathrm{MHz}^{\mathrm{CDCl}}\right)_{3}\right): \delta 6.82(\mathrm{~d}, 2 \mathrm{H})$, $3.59(\mathrm{q}, J=7.1 \mathrm{~Hz}, 0.9 \mathrm{H}), 3.54-3.48(\mathrm{~m}, 1.1 \mathrm{H}), 3.10(\mathrm{q}, J=7.1 \mathrm{~Hz}, 1.1 \mathrm{H}), 3.04-3.00(\mathrm{~m}, 0.9 \mathrm{H}), 2.26(\mathrm{~d}$, $3.0 \mathrm{H}), 2.20(\mathrm{~d}, 6.0 \mathrm{H}), 1.70-1.62(\mathrm{tt}, J=7.8,6.6 \mathrm{~Hz}, 1 \mathrm{H}), 1.45-1.36(\mathrm{~m}, 2 \mathrm{H}), 1.25(\mathrm{t}, J=7.1 \mathrm{~Hz}, 1.5 \mathrm{H})$, $1.17-1.07(\mathrm{~m}, 1 \mathrm{H}), 1.04-0.97(\mathrm{~m}, 3.0 \mathrm{H}), 0.77(\mathrm{t}, J=7.4 \mathrm{~Hz}, 1.5 \mathrm{H}) \mathrm{ppm} .{ }^{13} \mathrm{C}$ NMR $\left(151 \mathrm{MHz}, \mathrm{CDCl}_{3}\right): \delta$ 171.0, 170.9, 137.7, 134.4, 134.2, 133.5, 128.3 (2C), 47.6, 43.6, 42.7, 38.9, 30.7, 29.7, 21.2, 20.7, 20.1, 19.2, 19.1, 14.1, 13.9, 13.8, 12.8 ppm. IR (neat) $\mathbf{v}_{\text {max }}$ : 2959, 2931, 1627, 1612, 1455, 1417, 1377, 1287, 1099, 850, 748, 597, 584. $\left.\mathrm{HRMS} \mathrm{(ESI}^{+}\right)$: exact mass calculated for $\left[\mathrm{M}+\mathrm{H}^{+}\left(\mathrm{C}_{16} \mathrm{H}_{26} \mathrm{NO}^{+}\right)\right.$requires 248.2014 $\mathrm{m} / \mathrm{z}$ found $248.2016 \mathrm{~m} / \mathrm{z}$.

((3S,4R)-3-((Benzo[d][1,3]dioxol-5-yloxy)methyl)-4-(4-fluorophenyl)piperidin-1yl)(mesityl)methanone (1q)<smiles>Cc1cc(C)c(C(=O)N2CC[C@@H](c3ccc(F)cc3)[C@H](COc3ccc4c(c3)OCO4)C2)c(C)c1</smiles>

Paroxetine hydrochloride hemihydrate ( $329 \mathrm{mg}, 1 \mathrm{mmol}, 1.0$ eq.) was dissolved together with triethylamine $(418 \mu \mathrm{L}, 3.0$ eq. $)$ in dichloromethane $(3 \mathrm{~mL})$. The mixture was cooled down to $0{ }^{\circ} \mathrm{C}$ and 2,4,6-trimethylbenzoyl chloride $(500 \mu \mathrm{L}, 3.0$ eq.) was added dropwise. The reaction was allowed to come up to room temperature and was stirred for $16 \mathrm{~h}$. Then, the organic phase was washed with a $1 \mathrm{M} \mathrm{HCl}$ solution $(10 \mathrm{~mL})$ and brine solution $(10 \mathrm{~mL})$. The organic phase was dried over anhydrous $\mathrm{MgSO}_{4}$, concentrated and the crude material was subjected to column chromatography giving rise to the title compound as a white solid (152 mg, 32\%). NMR spectroscopy analysis revealed two rotamers in a ratio of 
around 1:1. Both rotamers are described together: ${ }^{1} \mathbf{H}$ NMR $\left(400 \mathrm{MHz}, \mathrm{CDCl}_{3}\right): \delta 7.16-7.09(\mathrm{~m}, 2 \mathrm{H})$, $7.03-6.96(\mathrm{~m}, 2 \mathrm{H}), 6.89-6.84(\mathrm{~m}, 2 \mathrm{H}), 6.64(\mathrm{~d}, J=8.5 \mathrm{~Hz}, 0.5 \mathrm{H}), 6.58(\mathrm{~d}, J=8.5 \mathrm{~Hz}, 0.5 \mathrm{H}), 6.39(\mathrm{~d}, J=$ $2.5 \mathrm{~Hz}, 0.5 \mathrm{H}$ ), 6.17 (dd, $J=8.5,2.5 \mathrm{~Hz}, 0.5 \mathrm{H}), 6.12(\mathrm{~d}, J=2.5 \mathrm{~Hz}, 0.5 \mathrm{H}), 5.94(\mathrm{dd}, J=8.5,2.5 \mathrm{~Hz}, 0.5 \mathrm{H})$, $5.89(\mathrm{~d}, J=4.4 \mathrm{~Hz}, 2 \mathrm{H}), 5.18(\mathrm{dd}, J=13.3,2.5 \mathrm{~Hz}, 0.5 \mathrm{H}), 5.07-4.98(\mathrm{~m}, 0.5 \mathrm{H}), 3.73-3.63(\mathrm{~m}, 1 \mathrm{H}), 3.58$ $-3.46(\mathrm{~m}, 1.5 \mathrm{H}), 3.39-3.32(\mathrm{~m}, 0.5 \mathrm{H}), 3.14-2.70(\mathrm{~m}, 3 \mathrm{H}), 2.36(\mathrm{~s}, 1.5 \mathrm{H}), 2.32(\mathrm{~s}, 1.5 \mathrm{H}), 2.29-2.27$ $\left.(\mathrm{m}, 3 \mathrm{H}), 2.21-2.18(\mathrm{~s}, 3 \mathrm{H}), 2.14-1.57(\mathrm{~m}, 3 \mathrm{H}) \mathrm{ppm} .{ }^{13} \mathrm{C} \mathrm{NMR} \mathrm{(101} \mathrm{MHz}, \mathrm{CDCl}_{3}\right): \delta 172.6,170.2,170.1$, 163.0 (2C), 160.6 (2C), 154.4, 153.6, 148.3 (2C), 142.0, 141.9, 139.6, 138.7, 138.6 (3C), 138.2, 138.1, $135.7(2 \mathrm{C}), 133.5(2 \mathrm{C}), 133.3,128.9(2 \mathrm{C}), 128.8(2 \mathrm{C}), 128.6(2 \mathrm{C}), 128.5(2 \mathrm{C}), 128.4(2 \mathrm{C}), 115.9(2 \mathrm{C}), 115.7$ (2C), 108.0, 107.9, 105.8, 105.7, 101.3, 101.2, 98.2 (2C), 68.7, 68.3, 49.5, 46.7, 44.5, 44.2, 43.9, 43.0, 42.4, 41.8, 34.8, 33.9, 21.3, 21.2 (2C), 19.5, 19.4, 19.1 (2C) ppm. $\left.{ }^{19} \mathrm{~F} \mathrm{NMR} \mathrm{(376} \mathrm{MHz,} \mathrm{CDCl}\right): \delta-115.7$, 115.8 ppm. IR (neat) $\mathbf{v}_{\max }: 2919,1609,1509,1487,1468,1445,1225,1178,1038,832.730$. HRMS $\left(\mathrm{ESI}^{+}\right)$: exact mass calculated for $[\mathrm{M}+\mathrm{H}]^{+}\left(\mathrm{C}_{29} \mathrm{H}_{31} \mathrm{FNO}_{4}{ }^{+}\right)$requires $\mathrm{m} / \mathrm{z} 476.2232$ found $\mathrm{m} / \mathrm{z} 476.2242$.

\section{(1-(3,4-Dimethoxybenzyl)-6,7-dimethoxy-3,4-dihydroisoquinolin-2(1H)-yl)(mesityl)methanone (1r)}<smiles>COc1ccc(CC2c3cc(OC)c(OC)cc3CCN2C(=O)c2c(C)cc(C)cc2C)cc1OC</smiles>
Norlaudanosine hydrochloride (570 mg, $1.50 \mathrm{mmol}, 1.00$ eq.) was dissolved in $5 \mathrm{~mL}$ of $\mathrm{CH}_{2} \mathrm{Cl}_{2}$ together with triethylamine $(418 \mu \mathrm{L}$, $3.00 \mathrm{mmol}, 2.00$ eq.). The mixture was cooled to $0{ }^{\circ} \mathrm{C}$ and $2,4,6-$ trimethylbenzoyl chloride ( $250 \mu \mathrm{L}, 1.5 \mathrm{mmol}, 1.0$ eq.) was added dropwise. The reaction was allowed to warm to room temperature and was stirred for $16 \mathrm{~h}$. Then, the organic phase was sequentially washed with a $1 \mathrm{M} \mathrm{HCl}$ solution $(10 \mathrm{~mL})$ and brine $(10 \mathrm{~mL})$. The organic phase was dried over $\mathrm{MgSO}_{4}$, concentrated and the crude material was subjected to column chromatography. In the column chromatography two compounds were isolated. Compound $\mathbf{A}\left(R_{f}=0.27\right.$ in EtOAc/heptane, 1:1): white solid, $433 \mathrm{mg}(59 \%)$. Compound B $\left(R_{f}=0.17\right.$ in EtOAc/heptane, 1:1): white solid, $174 \mathrm{mg}(24 \%)$. Under careful analysis of spectroscopic data, we concluded that both compounds correspond to the title compound. We assume that $\mathbf{A}$ and $\mathbf{B}$ are atropisomers. This assumption is supported by the observation that, overtime in solution (in $\mathrm{CDCl}_{3}$ over several hours), $\mathbf{A}$ and $\mathbf{B}$ formed an equilibrium (ratio: 2.9:1 (A:B)).

Compound A: ${ }^{1} \mathrm{H}$ NMR (400 MHz, $\left.\mathrm{CDCl}_{3}\right): \delta 6.87(\mathrm{~s}, 1 \mathrm{H}), 6.84(\mathrm{~s}, 1 \mathrm{H}), 6.80(\mathrm{~s}, 1 \mathrm{H}), 6.75(\mathrm{~d}, J=8.1 \mathrm{~Hz}$, $1 \mathrm{H}), 6.68(\mathrm{~d}, J=8.7 \mathrm{~Hz}, 1 \mathrm{H}), 6.55(\mathrm{~s}, 1 \mathrm{H}), 6.23(\mathrm{~s}, 1 \mathrm{H}), 5.99(\mathrm{t}, J=7.0 \mathrm{~Hz}, 1 \mathrm{H}), 3.86-3.80(\mathrm{~m}, 9 \mathrm{H}), 3.62$ $(\mathrm{s}, 3 \mathrm{H}), 3.40-3.22(\mathrm{~m}, 3 \mathrm{H}), 3.06(\mathrm{dd}, J=13.5,8.0 \mathrm{~Hz}, 1 \mathrm{H}), 2.78-2.69(\mathrm{~m}, 1 \mathrm{H}), 2.56(\mathrm{dt}, J=16.1,3.7 \mathrm{~Hz}$, $1 \mathrm{H}), 2.27(\mathrm{~s}, 3 \mathrm{H}), 2.14(\mathrm{~s}, 3 \mathrm{H}), 2.02(\mathrm{~s}, 3 \mathrm{H}) \mathrm{ppm} .{ }^{13} \mathrm{C}$ NMR (101 MHz, CDCl 3 ): $\delta 170.3,149.0,147.9(2 \mathrm{C})$, $147.1,138.0,133.8$ (2C), 133.5, 130.7, 128.6, 128.4, 128.2, 125.1, 122.2, 113.0, 111.2, 111.1, 110.9, $56.1,56.0,55.9,55.8,52.9,42.3,40.8,28.8,21.2,19.2,18.7$ ppm. IR (neat) $\mathbf{v}_{\text {max }}$ : 1627, 1610, 1513, 1439, 1418, 1256, 1224, 1173, 1156, 1138, 1125, 1106, 1027, 853. HRMS (ESI ${ }^{+}$): exact mass calculated for $[\mathrm{M}+\mathrm{Na}]^{+}\left(\mathrm{C}_{30} \mathrm{H}_{35} \mathrm{NO}_{5} \mathrm{Na}^{+}\right)$requires $\mathrm{m} / \mathrm{z} 512.2413$, found $\mathrm{m} / \mathrm{z} 512.2392$.

Compound B: ${ }^{1} \mathrm{H}$ NMR $\left(400 \mathrm{MHz}, \mathrm{CDCl}_{3}\right): \delta 6.93(\mathrm{~s}, 1 \mathrm{H}), 6.81(\mathrm{~s}, 1 \mathrm{H}), 6.66(\mathrm{~d}, J=8.1 \mathrm{~Hz}, 1 \mathrm{H}), 6.62(\mathrm{~s}$, $1 \mathrm{H}), 6.29(\mathrm{~d}, J=8.1 \mathrm{~Hz}, 1 \mathrm{H}), 6.06(\mathrm{~s}, 1 \mathrm{H}), 5.71(\mathrm{~s}, 1 \mathrm{H}), 4.90-4.80(\mathrm{~m}, 1 \mathrm{H}), 4.53(\mathrm{dd}, J=8.8,4.6 \mathrm{~Hz}, 1 \mathrm{H})$, $3.85(\mathrm{~s}, 3 \mathrm{H}), 3.81(\mathrm{~s}, 3 \mathrm{H}), 3.62(\mathrm{~s}, 4 \mathrm{H}), 3.46(\mathrm{~s}, 3 \mathrm{H}), 3.34-3.25(\mathrm{~m}, 1 \mathrm{H}), 3.10-2.96(\mathrm{~m}, 2 \mathrm{H}), 2.89-2.74$ (m, 2H), 2.29 (s, 3H), $\left.2.20(\mathrm{~s}, 3 \mathrm{H}), 1.87(\mathrm{~s}, 3 \mathrm{H}) \mathrm{ppm} .{ }^{13} \mathrm{C} \mathrm{NMR} \mathrm{(101} \mathrm{MHz}, \mathrm{CDCl}_{3}\right): \delta 170.1,149.1,148.0$ (2C), 146.6, 138.1, 134.3, 134.0, 133.7, 130.4, 128.7, 128.3, 128.2, 125.9, 122.1, 111.9, 111.5, 111.1, $110.2,60.5,59.4,56.1,55.9,55.7,43.9,36.1,28.0,21.2,19.4,18.7$ ppm. IR (neat) $v_{\text {max }}$ : 1630, 1611, 1514, 1440, 1416, 1256, 1226, 1156, 1138, 1123, 1106, 1027, 854. HRMS (ESI ${ }^{+}$): exact mass calculated for $[\mathrm{M}+\mathrm{H}]^{+}\left(\mathrm{C}_{30} \mathrm{H}_{35} \mathrm{NO}_{5} \mathrm{Na}^{+}\right)$requires $\mathrm{m} / \mathrm{z} 512.2413$, found $\mathrm{m} / \mathrm{z} 512.2396$.

\section{Pyrrolidin-1-yl(2,4,6-triisopropylphenyl)methanone (3a)}<smiles>CCCc1cc(C(C)C)c(C(=O)N2CCCC2)c(C(C)C)c1</smiles>

Following the GP2B (4 mmol scale) using 2,4,6-triisopropylbenzoic acid and pyrrolidine afforded the desired amide $(0.99 \mathrm{~g}, 82 \%)$ as white solid. ${ }^{1} \mathrm{H}$ NMR (400 MHz, $\left.\mathrm{CDCl}_{3}\right): \delta 6.98(\mathrm{~s}, 2 \mathrm{H}), 3.67(\mathrm{t}, J=7.1 \mathrm{~Hz}, 2 \mathrm{H}), 3.08(\mathrm{t}, J=6.7 \mathrm{~Hz}$, $2 \mathrm{H}$ ), $2.91-2.77(\mathrm{~m}, 3 \mathrm{H}), 1.95$ (quint, $6.7 \mathrm{~Hz}, 2 \mathrm{H}), 1.83$ (quint, $J=6.7 \mathrm{~Hz}, 2 \mathrm{H}$ ), $1.24-1.20(\mathrm{~m}, 18 \mathrm{H}) \mathrm{ppm} .{ }^{13} \mathrm{C}$ NMR $\left(101 \mathrm{MHz}, \mathrm{CDCl}_{3}\right): \delta 170.1,149.3,143.9$, 
$133.8,121.2,48.5,45.0,34.5,31.2,26.0,25.4,24.8,24.2,23.7$ ppm. IR (neat) $\mathbf{v}_{\text {max }}$ : 2958, 2931, 2869,

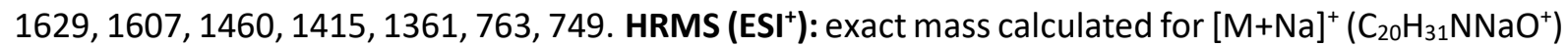
requires $\mathrm{m} / \mathrm{z} 324.2298$, found $\mathrm{m} / \mathrm{z} 324.2299$.

\section{Anthracen-9-yl(pyrrolidin-1-yl)methanone (3b)}<smiles>O=C(c1c2ccccc2cc2ccccc12)N1CCCC1</smiles>

Following the GP2B ( 2 mmol scale) using 9-anthracenecarboxylic acid and pyrrolidine afforded the desired amide $(0.36 \mathrm{~g}, 83 \%)$ as yellow solid. ${ }^{1} \mathbf{H}$ NMR $\left(400 \mathrm{MHz}, \mathrm{CDCl}_{3}\right)$ : $\delta 8.46(\mathrm{~s}, 1 \mathrm{H}), 8.04-8.00(\mathrm{~m}, 2 \mathrm{H}), 7.93(\mathrm{~d}, J=8.5 \mathrm{~Hz}, 2 \mathrm{H}), 7.54-7.45(\mathrm{~m}, 4 \mathrm{H}), 3.98$ $(\mathrm{t}, J=7.1 \mathrm{~Hz}, 2 \mathrm{H}), 2.93(\mathrm{t}, J=6.8 \mathrm{~Hz}, 2 \mathrm{H}), 2.08-2.01(\mathrm{~m}, 2 \mathrm{H}), 1.80$ (quint, $J=6.8 \mathrm{~Hz}$, $2 \mathrm{H}) \mathrm{ppm}$. Spectral data was found in agreement with the literature with one deviation (bold) in chemical shift. ${ }^{[5]}$

\section{Phenyl(pyrrolidin-1-yl)methanone (3c)}<smiles>O=C(c1ccccc1)N1CCCC1</smiles>

Following the GP1B ( $6 \mathrm{mmol}$ scale) using benzoyl chloride and pyrrolidine the desired amide (919 mg, 87\%) was obtained as colorless oil. ${ }^{1} \mathrm{H}$ NMR $(400 \mathrm{MHz}$, $\left.\mathrm{CDCl}_{3}\right) \delta 7.54-7.48(\mathrm{~m}, 2 \mathrm{H}), 7.43-7.37(\mathrm{~m}, 3 \mathrm{H}), 3.65(\mathrm{t}, J=6.9 \mathrm{~Hz}, 2 \mathrm{H}), 3.42(\mathrm{t}, J$ $=6.5 \mathrm{~Hz}, 2 \mathrm{H}), 2.03-1.83(\mathrm{~m}, 4 \mathrm{H}) \mathrm{ppm}$. Spectra data was found in agreement with the literature. ${ }^{[6]}$

\section{Phenyl(piperidin-1-yl)methanone (3d)}<smiles>O=C(c1ccccc1)N1CCCCC1</smiles>

Following the GP1A using benzoyl chloride and piperidine afforded the desired amide (1.06 g, 98\%) as colorless oil. ${ }^{1} \mathbf{H}$ NMR $\left(400 \mathrm{MHz}, \mathrm{CDCl}_{3}\right): \delta 7.41-7.37(\mathrm{~m}$, $5 \mathrm{H}), 3.80-3.26(\mathrm{~m}, 4 \mathrm{H}), 1.82-1.48(\mathrm{~m}, 6 \mathrm{H}) \mathrm{ppm}$. Spectral data was found in agreement with the literature. ${ }^{[7]}$

\section{Piperidin-1-yl(o-tolyl)methanone (3e)}<smiles>Cc1ccccc1C(=O)N1CCCCC1</smiles>

Following the GP1A using 2-methylbenzoyl chloride and piperidine afforded the desired amide (0.97 g, 95\%) as colorless oil. ${ }^{1} \mathrm{H}$ NMR (400 $\left.\mathbf{M H z}, \mathrm{CDCl}_{3}\right)$ : $\delta 7.17-$ $6.99(\mathrm{~m}, 4 \mathrm{H}), 3.74-3.51(\mathrm{~m}, 2 \mathrm{H}), 3.06-3.02(\mathrm{~m}, 2 \mathrm{H}), 2.18(\mathrm{~s}, 3 \mathrm{H}), 1.58-1.51(\mathrm{~m}$, $4 \mathrm{H}), 1.33$ (app s, 2H) ppm. Spectral data was found in agreement with the literature. ${ }^{[8]}$

\section{Piperidin-1-yl(p-tolyl)methanone (3f)}<smiles>Cc1ccc(C(=O)N2CCCCC2)cc1</smiles>

Following the GP1A using 4-methylbenzoyl chloride and piperidine afforded the desired amide (0.97 g, 95\%) as colorless oil. ${ }^{1} \mathbf{H}$ NMR (400 MHz, $\left.\mathrm{CDCl}_{3}\right): \delta 7.29$ (d, J = 7.9 Hz, 2H), 7.19 (d, J=7.8 Hz, 2H), 3.68 (app s, 2H), 3.36 (app s, 2H), 2.37 $(\mathrm{s}, 3 \mathrm{H}), 1.71-1.46(\mathrm{~m}, 6 \mathrm{H}) \mathrm{ppm}$. Spectral data was found in agreement with the literature. ${ }^{[9]}$

\section{(2-Methoxyphenyl)(piperidin-1-yl)methanone (3g)}<smiles>COc1ccccc1C(=O)N1CCCCC1</smiles>

Following the GP1A using 2-methoxybenzoyl chloride and piperidine afforded the desired amide (1.18 g, 99\%) as colorless oil. ${ }^{1} \mathrm{H}$ NMR (400 MHz, $\left.\mathrm{CDCl}_{3}\right): \delta 7.32$ (dd, $J=11.3,4.5 \mathrm{~Hz}, 1 \mathrm{H}), 7.21(\mathrm{dd}, \mathrm{J}=7.4,1.4 \mathrm{~Hz}, 1 \mathrm{H}), 6.97(\mathrm{t}, \mathrm{J}=7.5 \mathrm{~Hz}, 1 \mathrm{H}), 6.90(\mathrm{~d}, \mathrm{~J}$ $=8.3 \mathrm{~Hz}, 1 \mathrm{H}), 3.83(\mathrm{~s}, 3 \mathrm{H}), 3.80-3.63(\mathrm{~m}, 2 \mathrm{H}), 3.21-3.15(\mathrm{~m}, 2 \mathrm{H}), 1.72-1.58(\mathrm{~m}$, $6 \mathrm{H}) \mathrm{ppm}$. Spectral data was found in agreement with the literature. ${ }^{[0]}$ 
<smiles>COc1cccc(C(=O)N2CCCCC2)c1</smiles>

Following the GP1A using 3-methoxybenzoyl chloride and piperidine afforded the desired amide $(1.00 \mathrm{~g}, 91 \%)$ as colorless oil. ${ }^{1} \mathrm{H}$ NMR $(400 \mathrm{MHz}$, $\left.\mathrm{CDCl}_{3}\right): \delta 7.32-7.27(\mathrm{~m}, 1 \mathrm{H}), 6.96-6.90(\mathrm{~m}, 3 \mathrm{H}), 3.82(\mathrm{~s}, 2 \mathrm{H}), 3.67(\mathrm{~s}, 2 \mathrm{H})$, $3.34(\mathrm{~s}, 3 \mathrm{H}), 1.70-1.57(\mathrm{~m}, 6 \mathrm{H}) \mathrm{ppm}$. Spectral data was found in agreement with the literature. ${ }^{[8]}$

\section{(4-Methoxyphenyl)(piperidin-1-yl)methanone (3i)}<smiles>COc1ccc(C(=O)N2CCCCC2)cc1</smiles>

Following the GP1A using 4-methoxybenzoyl chloride and piperidine afforded the desired amide $(0.97 \mathrm{~g}, 89 \%)$ as colorless oil. ${ }^{1} \mathrm{H}$ NMR $(400 \mathrm{MHz}$, $\left.\mathrm{CDCl}_{3}\right): \delta 7.37(\mathrm{~d}, J=8.6 \mathrm{~Hz}, 2 \mathrm{H}), 6.90(\mathrm{~d}, J=8.6 \mathrm{~Hz}, 2 \mathrm{H}), 3.83(\mathrm{~s}, 3 \mathrm{H}), 3.75-$ $3.28(\mathrm{~m}, 4 \mathrm{H}), 1.74-1.58(\mathrm{~m}, J=37.6 \mathrm{~Hz}, 6 \mathrm{H}) \mathrm{ppm}$. Spectral data was found in agreement with the literature. ${ }^{[1]}$

Pyrrolidin-1-yl(3,4,5-trimethoxyphenyl)methanone (3j)<smiles>COc1cc(C(=O)N2CCCC2)cc(OC)c1OC</smiles>

Following the GP1A using 3,4,5-trimethoxybenzoyl chloride and pyrrolidine afforded the desired amide $(0.55 \mathrm{~g}, 42 \%)$ as colorless oil. ${ }^{1} \mathrm{H}$ NMR $(400 \mathrm{MHz}$, $\left.\mathrm{CDCl}_{3}\right)$ :) $\delta 6.76-6.71(\mathrm{~m}, 2 \mathrm{H}), 3.87-3.83(\mathrm{~m}, 9 \mathrm{H}), 3.66-3.57(\mathrm{~m}, 2 \mathrm{H}), 3.49$ $-3.40(\mathrm{~m}, 2 \mathrm{H}), 2.02-1.79(\mathrm{~m}, 4 \mathrm{H}) \mathrm{ppm} .{ }^{13} \mathrm{C} \mathrm{NMR}\left(101 \mathrm{MHz}, \mathrm{CDCl}_{3}\right): \delta 169.5$, 153.2, 139.4, 132.7, 104.7, 61.0, 56.4, 49.9, 46.4, 26.5, 24.6 ppm. IR (neat) $V_{\max }: 2967,2875,2835,1620,1579,1506,1452,1409,1343,1318,1233,1183,1119,1003,866,819$, 761, 707, 676. HRMS (ESI $\left.{ }^{+}\right)$: exact mass calculated for $[\mathrm{M}+\mathrm{H}]^{+}\left(\mathrm{C}_{14} \mathrm{H}_{20} \mathrm{NO}_{4}{ }^{+}\right)$requires $\mathrm{m} / \mathrm{z} 266.1387$, found $\mathrm{m} / \mathrm{z} 266.1389$.

Piperidin-1-yl(3,4,5-trimethoxyphenyl)methanone (3k)<smiles>COc1cc(C(=O)N2CCCCC2)cc(OC)c1OC</smiles>

Following the GP1A using 3,4,5-trimethoxybenzoyl chloride and piperidine afforded the desired amide $(0.81 \mathrm{~g}, 58 \%)$ as colorless oil. ${ }^{1} \mathrm{H}$ NMR $(400 \mathrm{MHz}$, $\left.\mathrm{CDCl}_{3}\right): \delta \delta 6.61(\mathrm{~s}, 2 \mathrm{H}), 3.90-3.80(\mathrm{~m}, 9 \mathrm{H}), 3.74-3.30(\mathrm{~m}, 4 \mathrm{H}), 1.77-1.50$ $(\mathrm{m}, 6 \mathrm{H}) \mathrm{ppm}$. Spectral data was found in agreement with the literature. ${ }^{[12]}$ 
<smiles>O=C(c1ccc2c(c1)OCO2)N1CCCCC1</smiles>

Following the GP1A using benzo[d][1,3]dioxole-5-carbonyl chloride and piperidine afforded the desired amide $(1.19 \mathrm{~g}, 89 \%)$ as colorless oil. ${ }^{1} \mathbf{H}$ NMR $\left(400 \mathrm{MHz}, \mathrm{CDCl}_{3}\right): \delta 6.93-6.87(\mathrm{~m}, 2 \mathrm{H}), 6.82-6.79(\mathrm{~m}, 1 \mathrm{H}), 5.99(\mathrm{~s}, 2 \mathrm{H}), 3.76$ $-3.30(\mathrm{~m}, 4 \mathrm{H}), 1.69-1.53(\mathrm{~m}, 6 \mathrm{H}) \mathrm{ppm}$. Spectral data was found in agreement with the literature. ${ }^{[13]}$

\section{(2-Bromo-5-methoxyphenyl)(pyrrolidin-1-yl)methanone (3m)}<smiles>COc1ccc(Br)c(C(=O)N2CCCC2)c1</smiles>

Following the GP2A using 2-bromo-5-methoxybenzoic acid and pyrrolidine afforded the desired amide $(1.25 \mathrm{~g}, 86 \%)$ as yellwoish oil. ${ }^{1} \mathbf{H}$ NMR $(400 \mathbf{~ M H z}$, $\left.\mathrm{CDCl}_{3}\right): \delta 7.42(\mathrm{~d}, J=8.7 \mathrm{~Hz}, 1 \mathrm{H}),, 6.81(\mathrm{~s}, 1 \mathrm{H}), 6.78(\mathrm{~d}, J=8.8 \mathrm{~Hz}, 1 \mathrm{H}), 3.78(\mathrm{~s}, 3 \mathrm{H})$, $3.65(\mathrm{t}, J=6.7 \mathrm{~Hz}, 2 \mathrm{H}), 3.24-3.16(\mathrm{~m}, 2 \mathrm{H}), 2.00-1.85(\mathrm{~m}, 4 \mathrm{H}) \mathrm{ppm} .{ }^{13} \mathrm{C}$ NMR (101 $\left.\mathrm{MHz} \mathrm{CDCl}_{3}\right): \delta 167.4,159.3,140.4,133.7,116.7,112.8,109.2,55.7,48.1,45.7$, 26.1, 24.7 ppm. IR (neat) $\mathbf{v}_{\text {max }}$ :2971, 2877, 2629, 1593, 1569, 1450, 1431, 1395, 1387, 1288, 1274, 1236, 1016, 820, 730, 639, 598. HRMS (ESI ${ }^{+}$): exact mass calculated for $[\mathrm{M}+\mathrm{H}]^{+}$ $\left(\mathrm{C}_{12} \mathrm{H}_{15} \mathrm{BrNO}_{2}^{+}\right)$requires $\mathrm{m} / \mathrm{z} 284.0286$, found $\mathrm{m} / \mathrm{z} 284.0267$.

\section{(4-Fluorophenyl)(pyrrolidin-1-yl)methanone (3n)}<smiles>O=C(c1ccc(F)cc1)N1CCCC1</smiles>

Following the GP1A using 4-fluorobenzoyl chloride and pyrrolidine afforded the desired amide $(0.72 \mathrm{~g}, 75 \%)$ as white solid. ${ }^{1} \mathrm{H}$ NMR $\left(\mathbf{4 0 0} \mathrm{MHz}, \mathrm{CDCl}_{3}\right): \delta 7.56-$ $7.47(\mathrm{~m}, 2 \mathrm{H}), 7.08-7.01(\mathrm{~m}, 2 \mathrm{H}), 3.62(\mathrm{t}, J=6.9 \mathrm{~Hz}, 2 \mathrm{H}), 3.41(\mathrm{t}, J=6.5 \mathrm{~Hz}, 2 \mathrm{H})$, $1.98-1.82(\mathrm{~m}, 4 \mathrm{H}) \mathrm{ppm}$. Spectral data was found in agreement with the literature. ${ }^{[14]}$

\section{(3-Chlorophenyl)(pyrrolidin-1-yl)methanone (30)}<smiles>O=C(c1cccc(Cl)c1)N1CCCC1</smiles>

Following the GP1A using 3-chlorobenzoyl chloride and pyrrolidine afforded the desired amide $(0.83 \mathrm{~g}, 79 \%)$ as colorless liquid. ${ }^{1} \mathrm{H} \mathbf{~ N M R}\left(600 \mathrm{MHz}, \mathrm{CDCl}_{3}\right): \delta 7.52-$ $7.48(\mathrm{~m}, 1 \mathrm{H}), 7.41-7.36(\mathrm{~m}, 2 \mathrm{H}), 7.36-7.31(\mathrm{~m}, 1 \mathrm{H}), 3.64(\mathrm{t}, J=6.9 \mathrm{~Hz}, 2 \mathrm{H}), 3.41$ $(\mathrm{t}, J=6.5 \mathrm{~Hz}, 2 \mathrm{H}), 2.01-1.84(\mathrm{~m}, 4 \mathrm{H}) \mathrm{ppm} .{ }^{13} \mathrm{C}$ NMR (151 MHz, $\left.\mathrm{CDCl}_{3}\right): \delta 168.3$, 139.1, 134.5, 130.0, 129.8, 127.5, 125.4, 49.7, 46.4, 26.5, 24.6 ppm. IR (neat) $\mathbf{v}_{\text {max: }}$ : 2972, 2876, 1620, 1565, 1427, 1400, 743, 702. HRMS (ESI ${ }^{+}$): exact mass calculated for $[\mathrm{M}+\mathrm{H}]^{+}\left(\mathrm{C}_{11} \mathrm{H}_{13} \mathrm{ClNO}{ }^{+}\right)$requires $\mathrm{m} / \mathrm{z} 210.0680$ found $\mathrm{m} / \mathrm{z} 210.684$.

\section{(2,3-Dihydro-1H-pyrrol-1-yl)(4-vinylphenyl)methanone (3p)}<smiles>C=Cc1ccc(C(=O)N2CCCC2)cc1</smiles>

Following the GP2A (2.8 mmol scale) using 4-vinylbenzoic acid and pyrrolidine afforded the desired amide $(0.39 \mathrm{~g}, 70 \%)$ as white solid. ${ }^{1} \mathbf{H}$ NMR (400 MHz, $\mathrm{CDCl}_{3}$ ): $\delta 7.49(\mathrm{~d}, J=8.2 \mathrm{~Hz}, 2 \mathrm{H}), 7.42(\mathrm{~d}, J=8.2 \mathrm{~Hz}, 2 \mathrm{H}), 6.72$ (dd, $J=17.6,10.9 \mathrm{~Hz}, 1 \mathrm{H}), 5.79(\mathrm{~d}, J=17.6 \mathrm{~Hz}, 1 \mathrm{H}), 5.30(\mathrm{~d}, J=10.9 \mathrm{~Hz}, 1 \mathrm{H}), 3.64$ $(\mathrm{t}, J=6.9 \mathrm{~Hz}, 2 \mathrm{H}), 3.43(\mathrm{t}, J=6.5 \mathrm{~Hz}, 2 \mathrm{H}), 2.01-1.82(\mathrm{~m}, 4 \mathrm{H}) \mathrm{ppm} .{ }^{13} \mathrm{C}$ NMR (101 MHz, $\left.\mathrm{CDCl}_{3}\right): \delta 169.5$, 139.1, 136.6, 136.3, 127.6, 126.1, 115.3, 49.7, 46.3, 26.6, 24.6 ppm. IR (neat) $\mathbf{v}_{\text {max }}$ : 2974, 2869, 1603, $1427,999,902,855,847,772,729$. RRMS $\left._{(E S I}{ }^{+}\right)$: exact mass calculated for $[\mathrm{M}+\mathrm{H}]^{+}\left(\mathrm{C}_{13} \mathrm{H}_{16} \mathrm{NO}^{+}\right)$requires $\mathrm{m} / \mathrm{z} 202.1226$ found $\mathrm{m} / \mathrm{z} 202.1228$. 
<smiles>CSc1ccc(C(=O)N2CCCC2)cc1</smiles>

Following the GP2A (1.6 mmol scale) using 4-(methylthio)benzoic acid and pyrrolidine afforded the desired amide $(0.28 \mathrm{~g}, 78 \%)$ as white solid. ${ }^{1} \mathbf{H}$ NMR $\left(400 \mathrm{MHz}, \mathrm{CDCl}_{3}\right): \delta 7.45(\mathrm{~d}, J=8.4 \mathrm{~Hz}, 2 \mathrm{H}), 7.23(\mathrm{~d}, J=8.4 \mathrm{~Hz}, 2 \mathrm{H}), 3.62(\mathrm{t}, J$ $=6.8 \mathrm{~Hz}, 2 \mathrm{H}), 3.44(\mathrm{t}, J=6.5 \mathrm{~Hz}, 2 \mathrm{H}), 2.48(\mathrm{~s}, 3 \mathrm{H}), 2.01-1.76(\mathrm{~m}, 4 \mathrm{H}) \mathrm{ppm} .{ }^{13} \mathrm{C}$ NMR (101 MHz, $\left.\mathrm{CDCl}_{3}\right): \delta 169.3,141.2,133.6,127.9,125.6,49.8,46.4,26.6$, 24.6, 15.4 ppm. IR (neat) $\mathbf{V}_{\text {max }}$ : 2969, 2866, 1593, 1550, 1420, 831, 752. HRMS (ESI ${ }^{+}$): exact mass calculated for $[\mathrm{M}+\mathrm{H}]^{+}\left(\mathrm{C}_{12} \mathrm{H}_{16} \mathrm{NOS}^{+}\right)$requires $\mathrm{m} / \mathrm{z} 222.0947$ found $\mathrm{m} / \mathrm{z} 222.0948$.

\section{3-(Pyrrolidine-1-carbonyl)benzonitrile (3r)}<smiles>N#Cc1cccc(C(=O)N2CCCC2)c1</smiles>

Following the GP2A using 3-cyanobenzoic acid and pyrrolidine afforded the desired amide $(0.27 \mathrm{~g}, 27 \%)$ as colorless oil. ${ }^{1} \mathrm{H} \mathbf{N M R}\left(\mathbf{4 0 0} \mathbf{M H z}, \mathrm{CDCl}_{3}\right): \delta 7.81(\mathrm{~d}, J=1.2 \mathrm{~Hz}$, $1 \mathrm{H}), 7.76(\mathrm{dd}, J=7.8,1.3 \mathrm{~Hz}, 1 \mathrm{H}), 7.70(\mathrm{dd}, J=7.8,1.2 \mathrm{~Hz}, 1 \mathrm{H}), 7.53(\mathrm{t}, J=7.8 \mathrm{~Hz}$, $1 \mathrm{H}$ ), $3.65(\mathrm{t}, J=6.8 \mathrm{~Hz}, 2 \mathrm{H}$ ), $3.41(\mathrm{t}, J=6.5 \mathrm{~Hz}, 2 \mathrm{H}$ ), 1.95 (app ddd, $J=19.2,13.2,6.7$ $\mathrm{Hz}, 4 \mathrm{H}) \mathrm{ppm} .{ }^{13} \mathrm{C} \mathrm{NMR}\left(101 \mathrm{MHz}, \mathrm{CDCl}_{3}\right): \delta 167.3,138.6,133.4,131.6,130.9,129.5$, $118.3,112.8,49.7,46.6,26.6,24.5$ ppm. IR (neat) $v_{\text {max }}$ : 2972, 2877, 2230, 1621, $1577,1435,1404,1340,1186,1173,912,815,797,745,723,691,628$. HRMS (ESI $\left.{ }^{+}\right)$: exact mass calculated for $[\mathrm{M}+\mathrm{H}]^{+}\left(\mathrm{C}_{12} \mathrm{H}_{13} \mathrm{~N}_{2} \mathrm{O}^{+}\right)$requires $\mathrm{m} / \mathrm{z} 201.1028$ found 201.1015.

\section{1-(Pyrrolidine-1-carbonyl)ferrocene (3u)}

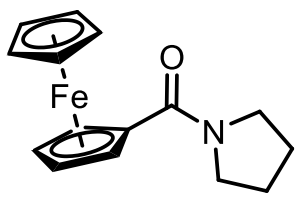

Following the GP2B $(0.7 \mathrm{mmol}$ scale) using ferrocenecarboxylic acid and pyrrolidine afforded the desired amide $(174 \mathrm{mg}, 88 \%)$ as orange solid. ${ }^{1} \mathbf{H}$ NMR (700 MHz, $\left.\mathrm{CDCl}_{3}\right): 84.73-4.72(\mathrm{~m}, 2 \mathrm{H}), 4.33-4.31(\mathrm{~m}, 2 \mathrm{H}), 4.20(\mathrm{~s}, 5 \mathrm{H}), 3.69(\mathrm{t}$, $J=6.2 \mathrm{~Hz}, 2 \mathrm{H}), 3.60(\mathrm{t}, J=6.4 \mathrm{~Hz}, 2 \mathrm{H}), 1.99-1.94(\mathrm{~m}, 2 \mathrm{H}), 1.92-1.88(\mathrm{~m}, 2 \mathrm{H})$ ppm. ${ }^{13} \mathrm{C}$ NMR (101 MHz, CDCl 3 ): $\delta$ 169.4, 78.0, 70.4, 70.0, 69.7, 48.2, 47.2, 26.9, $24.1 \mathrm{ppm}$. Spectral data was found in agreement with the literature. ${ }^{[15]}<$ sup $>[15]</$ sup $><$ sup $>[15]</$ sup $>$

\section{(4-Hydroxy-3-methoxyphenyl)(pyrrolidin-1-yl)methanone (Precursor for 3v)}<smiles>COc1cc(C(=O)O)ccc1O</smiles>
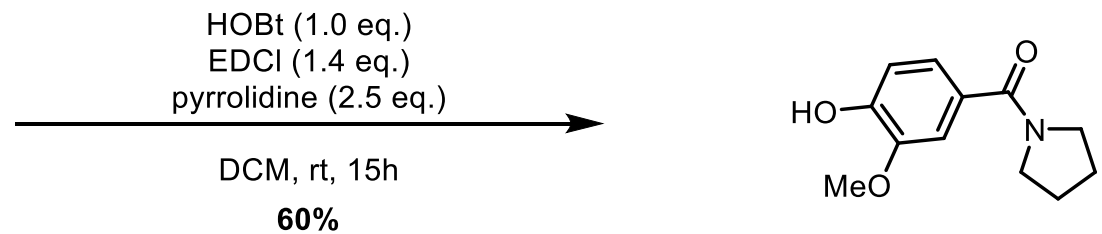

An oven-dried flask was loaded with (4-hydroxy-3-methoxyphenyl)(pyrrolidin-1-yl)methanone (1.68 g, $10 \mathrm{mmol}, 1.00$ eq.) and 1-hydroxybenzotriazole hydrate (1.35 g, $10 \mathrm{mmol}, 1.00$ eq.). Anhydrous $\mathrm{CH}_{2} \mathrm{Cl}_{2}$ $(10 \mathrm{~mL})$ was added, followed by pyrrolidine $(2.05 \mathrm{~mL}, 25 \mathrm{mmol}, 2.50$ eq. $)$ and 1-(3dimethylaminopropyl)-3-ethylcarbodiimide hydrochloride $(2.68 \mathrm{~g}, 14 \mathrm{mmol}, 1.40 \mathrm{eq}$.). The resulting mixture was stirred for $15 \mathrm{~h}$ at room temperature. Then, a saturated solution of $\mathrm{NaHCO}_{3}(10 \mathrm{~mL})$ was added and the aqueous phase was extracted with $\mathrm{CH}_{2} \mathrm{Cl}_{2}(3 \times 30 \mathrm{~mL})$. The organic phases were combined and dried over $\mathrm{MgSO}_{4}$. After concentration under reduced pressure, the crude material was subjected to column chromatography (heptane/EtOAc, 2:8 - pure EtOAc) to afford the title compound $(1.33 \mathrm{~g}, 6.0 \mathrm{mmol}, 60 \%)$ as white solid. ${ }^{1} \mathbf{H}$ NMR $\left(700 \mathrm{MHz}, \mathrm{CDCl}_{3}\right): \delta 7.15(\mathrm{~s}, 1 \mathrm{H}), 7.06(\mathrm{dd}, J=8.1$, $1.4 \mathrm{~Hz}, 1 \mathrm{H}), 6.89(\mathrm{~d}, J=8.1 \mathrm{~Hz}, 1 \mathrm{H}), 5.91(\mathrm{~s}, 1 \mathrm{H}), 3.91(\mathrm{~s}, 3 \mathrm{H}), 3.63(\mathrm{t}, J=6.5 \mathrm{~Hz}, 2 \mathrm{H}), 3.50(\mathrm{t}, J=6.0 \mathrm{~Hz}$, 2H), $1.97-1.85(\mathrm{~m}, 4 \mathrm{H}) \mathrm{ppm} \cdot{ }^{13} \mathrm{C}$ NMR (176 MHz, CDCl $): \delta$ 169.5, 147.4, 146.5, 129.1, 121.0, 113.7, $110.8,56.2,50.1,46.6,26.7,24.6$. IR (neat) $v_{\text {max }}$ : 1564, 1438, 1419, 1283, 1256, 1224, 1123, 1032, 878, 752, 726. HRMS (ESI $\left.{ }^{+}\right)$: exact mass calculated for $[\mathrm{M}+\mathrm{H}]^{+}\left(\mathrm{C}_{12} \mathrm{H}_{16} \mathrm{NO}_{3}{ }^{+}\right)$requires $\mathrm{m} / \mathrm{z} 222.1125$, found $\mathrm{m} / \mathrm{z} 222.1125$. 

carboxylate (3v)

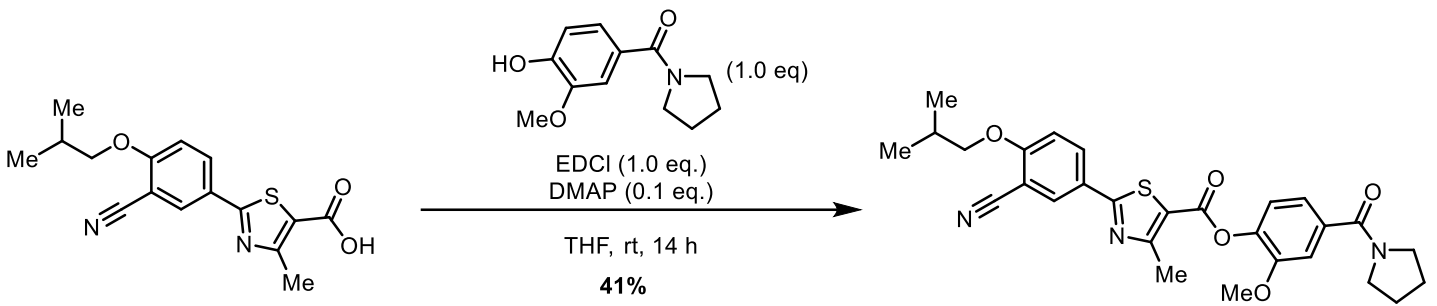

A modified procedure adopted from the literature was used. ${ }^{[16]}$ An oven-dried flask was loaded with (4-hydroxy-3-methoxyphenyl)(pyrrolidin-1-yl)methanone $(443 \mathrm{mg}, 2.0 \mathrm{mmol}, 1.00$ eq.), febuxostat (633 mg, $2.0 \mathrm{mmol}, 1.00$ eq.), 1-(3-dimethylaminopropyl)-3-ethylcarbodiimide hydrochloride (383 mg, $1.0 \mathrm{mmol}, 1.00$ eq.) and 4-dimethylaminopyridine $(24.4 \mathrm{mg}, 0.10 \mathrm{mmol}, 0.10$ eq.). Anhydrous tetrahydrofuran $(10 \mathrm{~mL})$ was added and the mixture was stirred for $14 \mathrm{~h}$ at room temperature. Then, a saturated solution of $\mathrm{NaHCO}_{3}$ was added and the aqueous phase was extracted with $\mathrm{CH}_{2} \mathrm{Cl}_{2}$ $(3 \times 10 \mathrm{~mL})$. The organic phases were combined and dried over $\mathrm{MgSO}_{4}$. After concentration under reduced pressure, the crude material was subjected to column chromatography (heptane/EtOAc, 8:2 $-2: 8$ ) to afford the title compound ( $425 \mathrm{mg}, 0.82 \mathrm{mmol}, 41 \%$ ) as white solid. ${ }^{1} \mathrm{H} \mathbf{N M R}\left(700 \mathrm{MHz}_{2} \mathrm{CDCl}_{3}\right)$ : $\delta 8.21(\mathrm{~d}, J=1.7 \mathrm{~Hz}, 1 \mathrm{H}), 8.11(\mathrm{dd}, J=8.6,1.8 \mathrm{~Hz}, 1 \mathrm{H}), 7.22(\mathrm{~s}, 1 \mathrm{H}), 7.16(\mathrm{~d}, J=8.0 \mathrm{~Hz}, 1 \mathrm{H}), 7.13-7.10$ $(\mathrm{m}, 1 \mathrm{H}), 7.02(\mathrm{~d}, J=8.9 \mathrm{~Hz}, 1 \mathrm{H}), 3.90(\mathrm{~d}, J=6.5 \mathrm{~Hz}, 2 \mathrm{H}), 3.85(\mathrm{~s}, 3 \mathrm{H}), 3.64(\mathrm{t}, J=6.9 \mathrm{~Hz}, 2 \mathrm{H}), 3.47(\mathrm{t}, J=$ $6.5 \mathrm{~Hz}, 2 \mathrm{H}), 2.80(\mathrm{~s}, 3 \mathrm{H}), 2.24-2.17(\mathrm{~m}, 1 \mathrm{H}), 1.99-1.94(\mathrm{~m}, 2 \mathrm{H}), 1.92-1.87(\mathrm{~m}, 2 \mathrm{H}), 1.08(\mathrm{~d}, J=6.7$ $\mathrm{Hz}, 6 \mathrm{H})$ ppm. ${ }^{13} \mathrm{C}$ NMR (176 MHz, $\left.\mathrm{CDCl}_{3}\right): \delta 168.9,168.3,163.2,162.8,159.8,151.4,140.4,136.3,132.8$, $132.3,126.0,122.7,120.4,119.6,115.4,112.8,112.1,103.2,75.8,56.2,49.9,46.5,28.3,26.5,24.5$, 19.1, 17.8 ppm. IR (neat) $v_{\text {max: }}$ 2966, 2876, 2230, 1731, 1605, 1508, 1428, 1253, 1170, 1121, 1050, 908, 726. HRMS (ESI $\left.{ }^{+}\right)$: exact mass calculated for $[\mathrm{M}+\mathrm{H}]^{+}\left(\mathrm{C}_{28} \mathrm{H}_{30} \mathrm{~N}_{3} \mathrm{O}_{5} \mathrm{~S}^{+}\right)$requires $\mathrm{m} / \mathrm{z} 520.1901$, found $\mathrm{m} / \mathrm{z}$ 520.1901.

\subsection{Characterization of Enamides}<smiles>Cc1cc(C)c(C(=O)N2C=CCCC2)c(C)c1</smiles>

\section{(3,4-Dihydropyridin-1(2H)-yl)(mesityl)methanone (2a)}

Following GP3 using 1a yielded the title compound $(61.1 \mathrm{mg}, 89 \%)$ as colorless oil. The desired enamide was obtained as two rotamers in a ratio of 3:1. Major isomer: ${ }^{1} \mathrm{H}$ NMR $\left(400 \mathrm{MHz}, \mathrm{CDCl}_{3}\right): \delta 6.84(\mathrm{~s}, 2 \mathrm{H}), 6.07(\mathrm{~d}, J=8.3 \mathrm{~Hz}, 1 \mathrm{H}), 4.86-4.76(\mathrm{~m}, 1 \mathrm{H}), 3.94-$ $3.84(\mathrm{~m}, 2 \mathrm{H}), 2.27(\mathrm{~s}, 3 \mathrm{H}), 2.17(\mathrm{~s}, 6 \mathrm{H}), 2.13-2.05(\mathrm{~m}, 2 \mathrm{H}), 1.94-1.85(\mathrm{~m}, 2 \mathrm{H}) \mathrm{ppm}$. Minor isomer: ${ }^{1} \mathrm{H}$ NMR $\left(400 \mathrm{MHz}, \mathrm{CDCl}_{3}\right): \delta 7.39(\mathrm{~d}, J=8.4 \mathrm{~Hz}, 1 \mathrm{H}), 6.84(\mathrm{~s}, 2 \mathrm{H}), 5.23-5.18(\mathrm{~m}, 1 \mathrm{H}), 3.26-3.20(\mathrm{~m}, 2 \mathrm{H})$, $2.27(\mathrm{~s}, 3 \mathrm{H}), 2.19(\mathrm{~s}, 6 \mathrm{H}), 2.13-2.06(\mathrm{~m}, 2 \mathrm{H}), 1.81-1.58(\mathrm{~m}, 2 \mathrm{H}) \mathrm{ppm}$. Major isomers: ${ }^{13} \mathrm{C}$ NMR (101 $\left.\mathrm{MHz}, \mathrm{CDCl}_{3}\right): \delta 169.6,138.5,134.0,132.9,128.3,125.9,108.5,40.1,22.1,21.6,21.2,19.0$ ppm. Minor isomer: $\left.{ }^{13} \mathrm{C} \mathrm{NMR} \mathrm{(101} \mathrm{MHz,} \mathrm{CDCl}_{3}\right): \delta 168.9,138.3,133.72,133.4,128.3,123.9,110.4,44.5,22.7,22.3$, 21.2, 19.0 ppm. IR (neat) $\mathbf{v}_{\text {max: }}$ 2921, 1664, 1632, 1612, 1434, 1406, 1372, 1355, 1293, 1257, 1172, 992, 844. HRMS (ESI $\left.{ }^{+}\right)$: exact mass calculated for $[\mathrm{M}+\mathrm{Na}]^{+}\left(\mathrm{C}_{15} \mathrm{H}_{19} \mathrm{NNaO}^{+}\right)$requires $\mathrm{m} / \mathrm{z} 252.1359$, found $\mathrm{m} / \mathrm{z}$ 252.1355 .

\section{(2,3-Dihydro-1H-pyrrol-1-yl)(mesityl)methanone (2b)}<smiles>Cc1cc(C)c(C(=O)N2C=CCC2)c(C)c1</smiles>

Following GP3 yielded the title compound $(36.0 \mathrm{mg}, 56 \%)$ as colorless oil. The desired enamide was obtained as 2 rotamers in a ratio of around 6:1. Major isomer: ${ }^{1} \mathrm{H}$ NMR $\left(600 \mathrm{MHz}, \mathrm{CDCl}_{3}\right): \delta 6.84(\mathrm{~s}, 2 \mathrm{H}), 5.90(\mathrm{dt}, J=4.4,2.2 \mathrm{~Hz}, 1 \mathrm{H})$, $5.13(\mathrm{dt}, J=4.4,2.6 \mathrm{~Hz}, 1 \mathrm{H}), 4.09-3.97(\mathrm{~m}, 2 \mathrm{H}), 2.73(\mathrm{ddt}, J=11.3,8.9,2.4 \mathrm{~Hz}$, 2), $2.27(\mathrm{~s}, 3 \mathrm{H}), 2.19(\mathrm{~s}, 6 \mathrm{H}) \mathrm{ppm}$. Minor isomer: ${ }^{1} \mathrm{H} \mathrm{NMR}\left(600 \mathrm{MHz}, \mathrm{CDCl}_{3}\right): \delta 7.13(\mathrm{dt}, J=4.4,2.2 \mathrm{~Hz}$, $1 \mathrm{H}), 6.85(\mathrm{~s}, 2 \mathrm{H}), 5.34(\mathrm{dt}, J=4.4,2.6 \mathrm{~Hz}, 1 \mathrm{H}), 3.43-3.34(\mathrm{~m}, 2 \mathrm{H}), 2.70-2.65(\mathrm{~m}, 2 \mathrm{H}), 2.27(\mathrm{~s}, 3 \mathrm{H}), 2.23$ 
(s, 6H) ppm. Major isomer: $\left.{ }^{13} \mathrm{C} \mathrm{NMR} \mathrm{(151} \mathrm{MHz,} \mathrm{CDCl}_{3}\right): \delta$ 167.6, 138.6, 134.1, 133.7, 129.9, 128.4, 112.1, 44.4, 28.9, 21.2, $19.1 \mathrm{ppm}$. Minor isomer: ${ }^{13} \mathrm{C}$ NMR (151 MHz, $\mathrm{CDCl}_{3}$ ): $\delta 167.1,138.4,134.3,133.3$, 129.0, 128.5, 112.1, 46.4, 30.0, 21.2, 19.0 ppm. IR (neat) $\mathbf{v}_{\max }$ : 3360, 2952, 2921, 1635, 1610, 1439, 1404, 1327, 1213, 1169, 1122, 1100, 1042, 998, 651, 826, 748. HRMS (ESI ${ }^{+}$): exact mass calculated for $[\mathrm{M}+\mathrm{Na}]^{+}\left(\mathrm{C}_{14} \mathrm{H}_{17} \mathrm{NNaO}^{+}\right)$requires $\mathrm{m} / \mathrm{z} 328.1202$, found $\mathrm{m} / \mathrm{z} 328.1199$.

\section{Mesityl(2,3,4,5-tetrahydro-1H-azepin-1-yl)methanone (2c)}

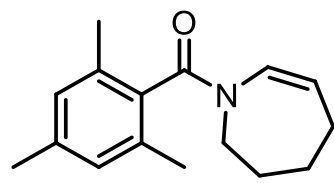

Following GP3 yielded the title compound ( $50.1 \mathrm{mg}, 69 \%$ ) as colorless oil. The desired enamide was obtained as two rotamers in a ratio of around 3.6:1. Major isomer: ${ }^{1} \mathrm{H} \mathrm{NMR}\left(400 \mathrm{MHz}, \mathrm{CDCl}_{3}\right): \delta 6.84(\mathrm{~s}, 2 \mathrm{H}), 5.90(\mathrm{~d}, J=8.5 \mathrm{~Hz}, 1 \mathrm{H})$, $4.98-4.87(\mathrm{~m}, 1 \mathrm{H}), 3.98($ app s, 2H), $2.31-2.18(\mathrm{~m}, 11 \mathrm{H}), 1.93($ app s, 2H), 1.77 (app s, 2H) ppm. Minor isomer: ${ }^{1} \mathrm{H}$ NMR (400 MHz, $\left.\mathrm{CDCl}_{3}\right): \delta 6.98(\mathrm{~d}, J=7.6 \mathrm{~Hz}, 1 \mathrm{H}), 6.85(\mathrm{~s}, 2 \mathrm{H})$, $5.33(\mathrm{~d}, J=5.2 \mathrm{~Hz}, 1 \mathrm{H}), 3.42($ app s, 2H), $2.35-2.15(\mathrm{~m}, 11 \mathrm{H}), 1.77$ (app s, 2H), 1.59 (app s, 2H) ppm. Major isomer: ${ }^{13} \mathrm{C}$ NMR (101 MHz, $\left.\mathrm{CDCl}_{3}\right)$ : $\delta$ 170.6, 138.3, 134.2, 133.6, 130.5, 128.3, 117.3, 45.2, 27.8, 26.4, 25.6, 21.2, $19.1 \mathrm{ppm}$. Minor isomer: ${ }^{13} \mathrm{C}$ NMR (101 MHz, $\left.\mathrm{CDCl}_{3}\right): \delta 170.9,138.2,133.8,133.4$, 129.5, 128.4, 118.3, 49.5, 30.4, 29.0, 26.6, 25.1, 19.1 ppm. IR (neat) $\mathbf{v}_{\text {max }}$ : 2927, 1636, 1612, 1440, 1405, 1382, 1365, 1351, 1340, 1275, 1257, 849, 765, 750. HRMS (ESI $\left.{ }^{+}\right)$: exact mass calculated for [M+Na] $]^{+}$ $\left(\mathrm{C}_{16} \mathrm{H}_{21} \mathrm{NNaO}^{+}\right)$requires $\mathrm{m} / \mathrm{z} 266.1515$, found $\mathrm{m} / \mathrm{z} 266.1515$.

\section{Mesityl(1,4-dioxa-8-azaspiro[4.5]dec-6-en-8-yl)methanone (2d)}<smiles>Cc1cc(C)c(C(=O)N2C=CC3(CC2)OCCO3)c(C)c1</smiles>

Following GP3 yielded the title compound ( $69.7 \mathrm{mg}, 81 \%$ ) as colorless oil. The desired enamide was obtained as two rotamers in a ratio of around 3.3:1. Major isomer: ${ }^{1} \mathrm{H}$ NMR $\left(700 \mathrm{MHz}, \mathrm{CDCl}_{3}\right): \delta 6.85(\mathrm{~s}, 2 \mathrm{H}), 6.25(\mathrm{~d}, J=$ $8.5 \mathrm{~Hz}, 1 \mathrm{H}), 4.76(\mathrm{~d}, J=8.4 \mathrm{~Hz}, 1 \mathrm{H}), 4.09-4.04(\mathrm{~m}, 2 \mathrm{H}), 4.04-3.97(\mathrm{~m}, 4 \mathrm{H})$, $2.27(\mathrm{~s}, 3 \mathrm{H}), 2.15(\mathrm{~s}, 6 \mathrm{H}), 2.06-2.00(\mathrm{~m}, 2 \mathrm{H}) \mathrm{ppm}$. Minor isomer: ${ }^{1} \mathrm{H}$ NMR $\left(700 \mathrm{MHz}^{\mathrm{CDCl}} \mathrm{CD}_{3}\right): \delta 7.56(\mathrm{~d}, J=8.6 \mathrm{~Hz}, 1 \mathrm{H}), 6.85(\mathrm{~s}, 2 \mathrm{H}), 5.14(\mathrm{~d}, J=8.6 \mathrm{~Hz}, 1 \mathrm{H}), 3.98-3.93(\mathrm{~m}, 4 \mathrm{H})$, $3.42-3.36(\mathrm{~m}, 2 \mathrm{H}), 2.27(\mathrm{~s}, 3 \mathrm{H}), 2.18(\mathrm{~s}, 6 \mathrm{H}), 1.91-1.87(\mathrm{~m}, 2 \mathrm{H}) \mathrm{ppm}$. Major isomer: ${ }^{13} \mathrm{C}$ NMR (176 $\left.\mathrm{MHz}, \mathrm{CDCl}_{3}\right): \delta$ 170.0, 139.0, 134.2, 132.0, 129.8, 128.5, 108.0, 103.6, 64.7, 38.6, 32.4, 21.2, 19.0 ppm. Minor isomer: ${ }^{13} \mathrm{C}$ NMR (176 MHz, $\left.\mathbf{C D C l}_{3}\right): \delta$ 169.2, 138.7, 133.7, 132.7, 128.4, 127.3, 109.8, 103.6, 64.7, 42.7, 33.4, 21.2, 19.0 ppm. IR (neat) $\mathbf{v}_{\text {max }}$ : 2881, 1665, 1632, 1591, 1369, 1293, 1224, 1170, 1119, $1041,853,745,564$. HRMS (ESI $\left.{ }^{+}\right)$: exact mass calculated for $[\mathrm{M}+\mathrm{H}]^{+}\left(\mathrm{C}_{17} \mathrm{H}_{22} \mathrm{NO}_{3}{ }^{+}\right)$requires 288.1600 $\mathrm{m} / \mathrm{z}$, found $288.1593 \mathrm{~m} / \mathrm{z}$.

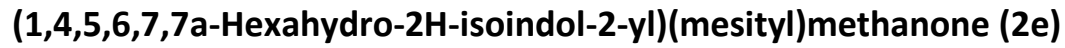

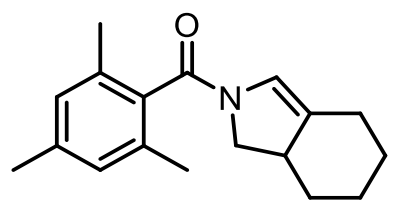

Following GP3 yielded the title compound $(55.6 \mathrm{mg}, 69 \%)$ as colorless oil. The desired enamide was obtained as two rotamers in a ratio of around 4.7:1. Both rotamers: ${ }^{1} \mathrm{H}$ NMR $\left(600 \mathrm{MHz}, \mathrm{CDCl}_{3}\right): \delta 6.86-6.82(\mathrm{~m}, 2 \mathrm{H}), 6.81$ $(\mathrm{t}, J=1.9 \mathrm{~Hz}, 0.2 \mathrm{H}), 5.56(\mathrm{t}, J=1.8 \mathrm{~Hz}, 0.8 \mathrm{H}), 4.22(\mathrm{dd}, J=12.8,10.1 \mathrm{~Hz}$, $0.8 \mathrm{H}), 3.56-3.49(\mathrm{~m}, 1 \mathrm{H}), 2.93(\mathrm{dd}, J=11.4,6.6 \mathrm{~Hz}, 0.2 \mathrm{H}), 2.84-2.76(\mathrm{~m}$, $J=33.4 \mathrm{~Hz}, 1 \mathrm{H}), 2.27-2.18(\mathrm{~m}, J=52.9 \mathrm{~Hz}, 9 \mathrm{H}), 2.08-2.03(\mathrm{~m}, J=16.0 \mathrm{~Hz}, 1 \mathrm{H}), 1.94-1.88(\mathrm{~m}, J=$ $34.7 \mathrm{~Hz}, 1 \mathrm{H}), 1.85-1.76(\mathrm{~m}, J=30.9 \mathrm{~Hz}, 2 \mathrm{H}), 1.40-1.10(\mathrm{~m}, 4 \mathrm{H}) \mathrm{ppm}$. Both rotamers: ${ }^{13} \mathrm{C}$ NMR (151 $\left.\mathrm{MHz}, \mathrm{CDCl}_{3}\right): \delta$ 166.6, 166.2, 138.2, 138.1, 134.3, 134.0, 134.0, 133.8, 133.2,133.1, 130.2, 130.1, 128.3, $128.2,120.2,119.6,53.2,50.9,43.1,42.2,34.7,34.4,27.4,27.2,25.9,25.7,25.3,25.2,21.1,19.0,18.9$ (3C) ppm. IR (neat) $\mathbf{v}_{\max }$ : 2927, 2853, 1630, 1580, 1445, 1333, 1319, 1211, 1173, 851, 811, 751. HRMS $\left(\mathrm{ESI}^{+}\right)$: exact mass calculated for $[\mathrm{M}+\mathrm{H}]^{+}\left(\mathrm{C}_{18} \mathrm{H}_{24} \mathrm{NO}^{+}\right)$requires $270.1858 \mathrm{~m} / \mathrm{z}$, found $270.1852 \mathrm{~m} / \mathrm{z}$. 
<smiles>Cc1cc(C)c(C(=O)N2C=COCC2)c(C)c1</smiles>

Following GP3 yielded the title compound as colorless oil $(27.7 \mathrm{mg}, 40 \%)$. In addition, remaining starting material $(20.7 \mathrm{mg}, 30 \%)$ was possible to be recovered. The desired enamide was obtained as two rotamers in a ratio of around 2.7:1. Major isomer: ${ }^{1} \mathrm{H}$ NMR $\left(700 \mathrm{MHz}, \mathrm{CDCl}_{3}\right): \delta 6.86(\mathrm{~s}, 2 \mathrm{H}), 5.79(\mathrm{~d}$, $J=4.6 \mathrm{~Hz}, 1 \mathrm{H}), 5.52(\mathrm{~d}, J=4.4 \mathrm{~Hz}, 1 \mathrm{H}), 4.17-4.13(\mathrm{~m}, 2 \mathrm{H}), 4.03-4.00(\mathrm{~m}, 2 \mathrm{H}), 2.28(\mathrm{~s}, 3 \mathrm{H}), 2.20-2.17$ $(\mathrm{m}, 6 \mathrm{H})$. Minor isomer: ${ }^{1} \mathrm{H}$ NMR $\left(700 \mathrm{MHz}, \mathrm{CDCl}_{3}\right): \delta 6.86(\mathrm{~s}, 2 \mathrm{H}), 6.83(\mathrm{~d}, J=4.6 \mathrm{~Hz}, 1 \mathrm{H}), 6.19(\mathrm{~d}, J=4.7$ $\mathrm{Hz}, 1 \mathrm{H}), 4.01(\mathrm{~s}, 2 \mathrm{H}), 3.37-3.34(\mathrm{~m}, 2 \mathrm{H}), 2.28(\mathrm{~s}, 3 \mathrm{H}), 2.20-2.17(\mathrm{~m}, 6 \mathrm{H}) \mathrm{ppm}$. Major isomer: ${ }^{13} \mathrm{C}$ NMR (176 MHz, $\mathrm{CDCl}_{3}$ ): $\delta 167.7,138.9,134.2,131.9,123.0,128.4,106.3,64.9,39.4,21.3,19.0$ ppm. Minor isomer: $\left.{ }^{13} \mathrm{C} \mathrm{NMR} \mathrm{(176} \mathrm{MHz}, \mathrm{CDCl}_{3}\right): \delta$ 167.2, 138.8, 134.0, 132.5, 132.1, 128.6, 104.5, 65.1, 44.0, 21.2, $19.0 \mathrm{ppm}$. IR (neat) $\mathbf{v}_{\max }: 1633,1409,1379,1215,903,852,723,649$. HRMS (ESI ${ }^{+}$): exact mass calculated for $[\mathrm{M}+\mathrm{H}]^{+}\left(\mathrm{C}_{14} \mathrm{H}_{18} \mathrm{NO}_{2}{ }^{+}\right)$requires $\mathrm{m} / \mathrm{z} 232.1332$ found $\mathrm{m} / \mathrm{z} 232.1328$.

\section{Ethyl 4-(2,4,6-trimethylbenzoyl)-3,4-dihydropyrazine-1(2H)-carboxylate (2g)}

Following GP3 yielded the title compound $(37.5 \mathrm{mg}, 41 \%)$ as colorless oil. In addition, remaining starting material ( $53.0 \mathrm{mg}, 58 \%$ ) was possible to be recovered. The desired enamide was obtained as four rotamers in a ratio of $4.8(A): 4(B): 1.9(C): 1(D)$.

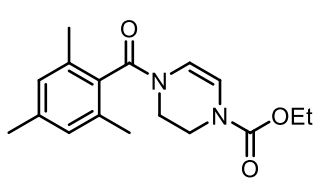

A or B

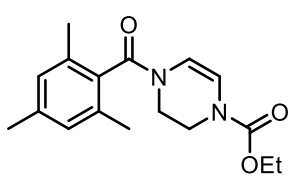

A or $B$

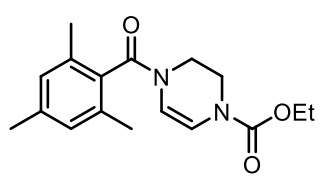

C or D

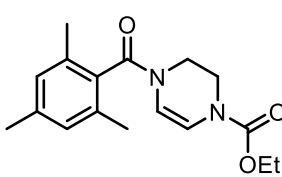

C or D

Rotamer A: ${ }^{1} \mathrm{H}$ NMR $\left(700 \mathrm{MHz}, \mathrm{CDCl}_{3}\right): \delta 6.86(\mathrm{~s}, 2 \mathrm{H}), 6.08(\mathrm{~d}, J=6.7 \mathrm{~Hz}, 1 \mathrm{H}), 5.49(\mathrm{~d}, J=6.7 \mathrm{~Hz}, 1 \mathrm{H})$, $4.24-4.20(\mathrm{~m}, 2 \mathrm{H}), 4.03-4.02(\mathrm{~m}, 2 \mathrm{H}), 3.83-3.77(\mathrm{~m}, 2 \mathrm{H}), 2.29(\mathrm{~s}, 3 \mathrm{H}), 2.17(\mathrm{~s}, 6 \mathrm{H}), 1.32-1.26(\mathrm{~m}$, 3H) ppm. ${ }^{13} \mathrm{C}$ NMR (176 MHz, $\left.\mathrm{CDCl}_{3}\right): \delta 168.4,152.3,139.0,134.2,132.1,128.5,109.3,108.2,62.5$, 41.0, 38.9, 21.3, 19.1, $14.7 \mathrm{ppm}$.

Rotamer B: ${ }^{1} \mathrm{H}$ NMR $\left(700 \mathrm{MHz}, \mathrm{CDCl}_{3}\right): \delta 6.80(\mathrm{~s}, 2 \mathrm{H}), 6.17(\mathrm{~d}, J=6.6 \mathrm{~Hz}, 1 \mathrm{H}), 5.51(\mathrm{~d}, J=6.6 \mathrm{~Hz}, 1 \mathrm{H})$, $4.20-4.09(\mathrm{~m}, 2 \mathrm{H}), 3.97-3.91(\mathrm{~m}, 2 \mathrm{H}), 3.74-3.71(\mathrm{~m}, 2 \mathrm{H}), 2.22(\mathrm{~s}, 3 \mathrm{H}), 2.11(\mathrm{~s}, 6 \mathrm{H}), 1.28-1.18(\mathrm{~m}$, 3H) ppm. ${ }^{13} \mathrm{C}$ NMR (176 MHz, $\left.\mathrm{CDCl}_{3}\right): \delta 168.5,152.6,139.0,134.2,132.1,128.5,110.0,108.9,62.5$, 41.6, 38.6, 21.3, 19.1, $14.7 \mathrm{ppm}$.

Rotamer C: ${ }^{1} \mathrm{H}$ NMR $\left(700 \mathrm{MHz}, \mathrm{CDCl}_{3}\right): \delta 6.86(\mathrm{~s}, 2 \mathrm{H}), 6.81(\mathrm{~d}, J=6.9 \mathrm{~Hz}, 1 \mathrm{H}), 6.48(\mathrm{~d}, J=6.9 \mathrm{~Hz}, 1 \mathrm{H})$, $4.24-4.19(\mathrm{~m}, 2 \mathrm{H}), 3.69-3.66(\mathrm{~m}, 2 \mathrm{H}), 3.39-3.35(\mathrm{~m}, 2 \mathrm{H}), 2.28(\mathrm{~s}, 3 \mathrm{H}), 2.17(\mathrm{~s}, 6 \mathrm{H}), 1.33-1.25(\mathrm{~m}$, $3 \mathrm{H})$ ppm. ${ }^{13} \mathrm{C}$ NMR (176 $\mathrm{MHz}, \mathrm{CDCl}_{3}$ ): only following signals could be clearly assigned to rotamer $\mathrm{C}: \delta$ $167.9,111.5,106.5,43.5,41.6 \mathrm{ppm}$.

Rotamer D: ${ }^{1} \mathrm{H}$ NMR $\left(700 \mathrm{MHz}, \mathrm{CDCl}_{3}\right): \delta 6.92-6.90(\mathrm{~m}, 1 \mathrm{H}), 6.86(\mathrm{~s}, 2 \mathrm{H}), 6.62(\mathrm{~d}, J=6.5 \mathrm{~Hz}, 1 \mathrm{H}), 4.24$ - $4.19(\mathrm{~m}, 2 \mathrm{H}), 3.69-3.66(\mathrm{~m}, 2 \mathrm{H}), 3.39-3.35(\mathrm{~m}, 2 \mathrm{H}), 2.28(\mathrm{~s}, 3 \mathrm{H}), 2.17(\mathrm{~s}, 6 \mathrm{H}), 1.33-1.25(\mathrm{~m}$, $3 \mathrm{H})$ ppm. ${ }^{13} \mathrm{C}$ NMR (176 $\mathrm{MHz}, \mathrm{CDCl}_{3}$ ): only following signals could be clearly assigned to rotamer $\mathrm{D}: \delta$ $112.1,107.3 \mathrm{ppm}$.

IR (neat) v $\max _{\text {: }}$ 2980, 2922, 1887, 1710, 1648, 1612, 1416, 1374, 1343, 1275, 1227, 1117, 993. HRMS $\left(\mathrm{ESI}^{+}\right)$: exact mass calculated for $[\mathrm{M}+\mathrm{H}]^{+}\left(\mathrm{C}_{17} \mathrm{H}_{23} \mathrm{~N}_{2} \mathrm{O}_{3}{ }^{+}\right)$requires $303.1709 \mathrm{~m} / \mathrm{z}$, found $303.1702 \mathrm{~m} / \mathrm{z}$.

\section{$\mathrm{N}$-Ethyl-2,4,6-trimethyl-N-vinylbenzamide (2h)}

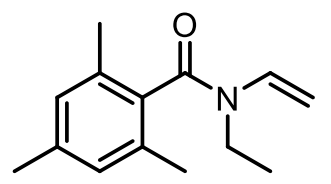

Following GP3 yielded the title compound $(50.9 \mathrm{mg}, 78 \%$ ) as orange oil. The desired enamide was obtained as two rotamers in a ratio of around 9:1. Major isomer: ${ }^{1} \mathbf{H}$ NMR (400 MHz, $\left.\mathrm{CDCl}_{3}\right): \delta 6.85(\mathrm{~s}, 2 \mathrm{H}), 6.30$ (dd, $\left.J=15.6,9.3 \mathrm{~Hz}, 1 \mathrm{H}\right)$, $4.49(\mathrm{~d}, J=15.7 \mathrm{~Hz}, 1 \mathrm{H}), 4.16(\mathrm{~d}, J=9.3 \mathrm{~Hz}, 1 \mathrm{H}), 3.89(\mathrm{q}, J=7.1 \mathrm{~Hz}, 2 \mathrm{H}), 2.28(\mathrm{~s}$,

$3 \mathrm{H}), 2.15(\mathrm{~s}, 6 \mathrm{H}), 1.26(\mathrm{t}, J=7.1 \mathrm{~Hz}, 3 \mathrm{H}) \mathrm{ppm}$. Minor isomer: ${ }^{1} \mathrm{H}$ NMR $\left(400 \mathrm{MHz}, \mathrm{CDCl}_{3}\right): \delta 7.61(\mathrm{dd}, J=$ 16.3, 9.5 Hz, 1H), $6.86(\mathrm{~s}, 2 \mathrm{H}), 4.59(\mathrm{~d}, J=10.9 \mathrm{~Hz}, 1 \mathrm{H}), 4.56(\mathrm{~d}, J=3.5 \mathrm{~Hz}, 1 \mathrm{H}), 3.39(\mathrm{q}, J=7.1 \mathrm{~Hz}, 2 \mathrm{H})$, $2.26(\mathrm{~s}, 3 \mathrm{H}), 2.20(\mathrm{~s}, 6 \mathrm{H}), 1.02(\mathrm{t}, J=7.1 \mathrm{~Hz}, 3 \mathrm{H}) \mathrm{ppm}$. Major isomer: ${ }^{13} \mathrm{C} \mathrm{NMR}\left(151 \mathrm{MHz}, \mathrm{CDCl}_{3}\right): \delta 170.5$, 
$138.6,134.0,133.0,132.9,128.4,93.2,36.1,21.2,18.9,11.8$ ppm. Minor isomer: ${ }^{13} \mathrm{C}$ NMR could not be clearly identified. IR (neat) $\mathbf{v}_{\text {max }}$ : 2935, 2921, 1667, 1618, 1461, 1446, 1425, 1345, 1310, 1076, 848, 607. HRMS (ESI $\left.{ }^{+}\right)$: exact mass calculated for $[\mathrm{M}+\mathrm{H}]^{+}\left(\mathrm{C}_{14} \mathrm{H}_{20} \mathrm{NO}^{+}\right)$requires $218.1545 \mathrm{~m} / \mathrm{z}$, found $218.1537 \mathrm{~m} / \mathrm{z}$.

(E)-2,4,6-Trimethyl-N-(prop-1-en-1-yl)-N-propylbenzamide (2i)<smiles>C/C=C/N(CCC)C(=O)c1c(C)cc(C)cc1C</smiles>

Following GP3 yielded the title compound ( $35.7 \mathrm{mg}, 49 \%$ ) as colorless oil. The desired enamide was obtained as two rotamers in a ratio of around 7.8:1. Major isomer: ${ }^{1} \mathrm{H}$ NMR (400 MHz, $\left.\mathrm{CDCl}_{3}\right): \delta 6.84(\mathrm{~s}, 2 \mathrm{H}), 6.05$ (dd, $J=14.1,1.3$ $\mathrm{Hz}, 1 \mathrm{H}), 5.01(\mathrm{dq}, J=13.4,6.6 \mathrm{~Hz}, 1 \mathrm{H}), 3.79-3.69(\mathrm{~m}, 2 \mathrm{H}), 2.29(\mathrm{~s}, 3 \mathrm{H}), 2.14$ $(\mathrm{s}, 6 \mathrm{H}), 1.75-1.65(\mathrm{~m}, 2 \mathrm{H}), 1.54(\mathrm{dd}, J=6.6,1.2 \mathrm{~Hz}, 3 \mathrm{H}), 0.99(\mathrm{t}, J=7.4 \mathrm{~Hz}$, 3H) ppm. Minor isomer: ${ }^{1} \mathbf{H}$ NMR (400 MHz, $\left.\mathbf{C D C l}_{3}\right): \delta 7.37(\mathrm{~d}, J=14.7 \mathrm{~Hz}, 1 \mathrm{H}), 6.81(\mathrm{~s}, 2 \mathrm{H}), 5.13$ (dq, J $=13.2,6.6 \mathrm{~Hz}, 1 \mathrm{H}), 3.27-3.15(\mathrm{~m}, 2 \mathrm{H}), 2.29(\mathrm{~s}, 3 \mathrm{H}), 2.19(\mathrm{~s}, 6 \mathrm{H}), 1.79(\mathrm{dd}, J=6.6,1.3 \mathrm{~Hz}, 3 \mathrm{H}), 1.74-$ $1.64(\mathrm{~m}, 2 \mathrm{H}), 0.71(\mathrm{t}, J=7.4 \mathrm{~Hz}, 3 \mathrm{H}) \mathrm{ppm}$. Major isomer: $\left.{ }^{13} \mathrm{C} \mathrm{NMR} \mathrm{(101} \mathrm{MHz}, \mathrm{CDCl}_{3}\right): \delta 170.1,138.3$, 133.9, 133.5, 128.4, 127.9, 105.8, 43.8, 21.3, 20.1, 19.0, 15.6, 11.7 ppm. Minor isomer: ${ }^{13} \mathrm{C}$ NMR (101 MHz, $\mathrm{CDCl}_{3}$ ): $\delta 169.8,138.3,133.7,133.6,128.5,125.8,107.9,47.9,20.7,19.3,15.8,11.5$ ppm. One peak could not be detected presumably due to an overlapping with a peak of the major species. IR (neat) $\mathbf{v}_{\text {max }}$ : 2960, 2935, 1640, 1612, 1443, 1398, 1373, 1323, 1226, 1084, 850. HRMS (ESI ${ }^{+}$): exact mass calculated for $[\mathrm{M}+\mathrm{Na}]^{+}\left(\mathrm{C}_{16} \mathrm{H}_{23} \mathrm{NNaO}^{+}\right)$requires $\mathrm{m} / \mathrm{z} 268.1672$, found $\mathrm{m} / \mathrm{z} 268.1668$.

(E)-N-(But-1-en-1-yl)-N-butyl-2,4,6-trimethylbenzamide (2j)

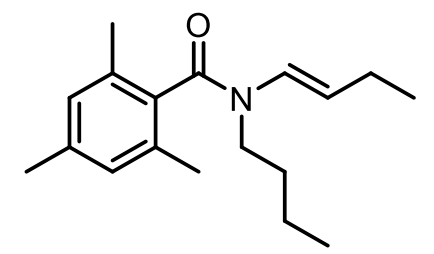

Following GP3 yielded the title compound (29.4 mg, 48\%) as colorless oil. The desired enamide was obtained as two rotamers in a ratio of around 7.4:1. Both rotamers are described together: ${ }^{1} \mathrm{H} \mathbf{~ N M R ~}\left(600 \mathrm{MHz}, \mathrm{CDCl}_{3}\right): \delta$ $7.37(\mathrm{~d}, J=14.7 \mathrm{~Hz}, 0.1 \mathrm{H}), 6.84(\mathrm{~s}, 1.8 \mathrm{H}), 6.81(\mathrm{~s}, 0.2 \mathrm{H}), 6.04(\mathrm{~d}, J=14.1 \mathrm{~Hz}$, $0.9 \mathrm{H}), 5.18-5.13(\mathrm{~m}, 0.1 \mathrm{H}), 5.04(\mathrm{dt}, J=14.0,6.9 \mathrm{~Hz}, 0.9 \mathrm{H}), 3.81-3.75$ $(\mathrm{m}, 1.8 \mathrm{H}), 3.26-3.23(\mathrm{~m}, 0.2 \mathrm{H}), 2.29-2.26(\mathrm{~m}, J=13.0 \mathrm{~Hz}, 3 \mathrm{H}), 2.20-$ $2.14(\mathrm{~m}, 6 \mathrm{H}), 1.92-1.85(\mathrm{~m}, 2 \mathrm{H}), 1.68-1.63(\mathrm{~m},, 2 \mathrm{H}), 1.47-1.38(\mathrm{~m}, 2 \mathrm{H}), 0.99(\mathrm{t}, \mathrm{J}=7.4 \mathrm{~Hz}, 3 \mathrm{H}), 0.88$ (t, $J=7.4 \mathrm{~Hz}, 3 \mathrm{H}) \mathrm{ppm} .{ }^{13} \mathrm{C}$ NMR $\left(151 \mathrm{MHz}, \mathrm{CDCl}_{3}\right): \delta 170.2,138.3,133.9,133.5,128.4,126.7,113.2$, 42.0, 28.8, 23.7, 21.3, 20.6, 19.0, 15.0, 14.0 ppm. IR (neat) $v_{\text {max }}$ : 2959, 2931, 2872, 1395, 1325, 1090, 906, 729. HRMS (ESI $\left.{ }^{+}\right)$: exact mass calculated for $[\mathrm{M}+\mathrm{H}]^{+}\left(\mathrm{C}_{18} \mathrm{H}_{28} \mathrm{NO}^{+}\right)$requires $\mathrm{m} / \mathrm{z} 274.2165$ found $\mathrm{m} / \mathrm{z}$ 274.2162 .

\section{(2-Benzyl-3,4-dihydropyridin-1(2H)-yl)(mesityl)methanone (2k)}<smiles>Cc1cc(C)c(C(=O)N2C=CCCC2Cc2ccccc2)c(C)c1</smiles>

Following GP3 yielded the title compound (91.4 mg, 95\%) as yellowish oil. The desired enamide was obtained as two rotamers in a ratio of around 10:1. Only the major rotamer is described: ${ }^{1} \mathrm{H}$ NMR $\left(600 \mathrm{MHz}, \mathrm{CDCl}_{3}\right): \delta$ $7.36(\mathrm{~d}, J=7.1 \mathrm{~Hz}, 2 \mathrm{H}), 7.34-7.30(\mathrm{~m}, 2 \mathrm{H}), 7.24(\mathrm{t}, J=7.2 \mathrm{~Hz}, 1 \mathrm{H}), 6.85(\mathrm{~s}$, $1 \mathrm{H}), 6.08(\mathrm{~d}, J=8.4 \mathrm{~Hz}, 1 \mathrm{H}), 5.15-5.10(\mathrm{~m}, 1 \mathrm{H}), 4.89-4.84(\mathrm{~m}, 1 \mathrm{H}), 3.10$ (dd, $J=13.1,4.9 \mathrm{~Hz}, 1 \mathrm{H}), 2.76(\mathrm{dd}, J=13.0,10.8 \mathrm{~Hz}, 1 \mathrm{H}), 2.29(\mathrm{~s}, 3 \mathrm{H}), 2.28$ $-2.24(\mathrm{~m}, 1 \mathrm{H}), 2.18(\mathrm{~s}, 3 \mathrm{H}), 2.14(\mathrm{~s}, 3 \mathrm{H}), 2.01(\mathrm{dt}, J=18.1,5.3 \mathrm{~Hz}, 1 \mathrm{H}), 1.79-1.75(\mathrm{~m}, 1 \mathrm{H}), 1.65-1.58$ (m, 1H) ppm. ${ }^{13} \mathrm{C}$ NMR (151 MHz, $\left.\mathrm{CDCl}_{3}\right): \delta$ 169.2, 138.5, 138.4, 134.2, 134.1, 133.0, 129.6, 128.6, 128.4, $128.3,126.5,124.8,107.7,49.7,36.2,22.0,21.3,19.0,18.9,18.2$ ppm. IR (neat) $\mathbf{v}_{\text {max }}$ : 1627, 1412, 1358, $1270,986,852,766,731,699,514$. HRMS $\left._{(E S I}\right)$ : exact mass calculated for $[\mathrm{M}+\mathrm{H}]^{+}\left(\mathrm{C}_{22} \mathrm{H}_{26} \mathrm{NO}^{+}\right)$requires $\mathrm{m} / \mathrm{z} 320.2014$, found $\mathrm{m} / \mathrm{z} 320.2006$. 
<smiles>[CH]C1CCC=CN1C(=O)c1c(C)cc(C)cc1C</smiles>

Following GP3 yielded the title compound $(60.5 \mathrm{mg}, 83 \%)$ as colorless oil. The desired enamide was obtained as two rotamers in a ratio of around 9:1. Major isomer: ${ }^{1} \mathrm{H}$ NMR $\left(600 \mathrm{MHz}, \mathrm{CDCl}_{3}\right)$ : $\delta 6.83(\mathrm{~s}, 2 \mathrm{H}), 6.04-5.95(\mathrm{~m}, 1 \mathrm{H}), 5.03-4.91$ $(\mathrm{m}, 1 \mathrm{H}), 4.82-4.72(\mathrm{~m}, 1 \mathrm{H}), 2.29-2.24(\mathrm{~m}, 3 \mathrm{H}), 2.22-2.12(\mathrm{~m}, 7 \mathrm{H}), 2.05-1.98$ $(\mathrm{m}, 1 \mathrm{H}), 1.84-1.76(\mathrm{~m}, 2 \mathrm{H}), 1.24(\mathrm{~d}, J=6.7 \mathrm{~Hz}, 3 \mathrm{H}) \mathrm{ppm}$. Minor isomer: ${ }^{1} \mathrm{H}$ NMR $\left(600 \mathrm{MHz}, \mathrm{CDCl}_{3}\right): \delta$ $7.32(\mathrm{ddt}, J=8.5,2.5,1.3 \mathrm{~Hz}, 1 \mathrm{H}), 6.88(\mathrm{~s}, 2 \mathrm{H}), 5.21-5.18(\mathrm{~m}, 1 \mathrm{H}), 3.75-3.66(\mathrm{~m}, 1 \mathrm{H}), 2.29-2.24(\mathrm{~m}$, $3 \mathrm{H}), 2.23-2.12(\mathrm{~m}, 7 \mathrm{H}), 2.04-1.94(\mathrm{~m}, 1 \mathrm{H}), 1.83-1.71(\mathrm{~m}, 2 \mathrm{H}), 0.98(\mathrm{~d}, J=6.6 \mathrm{~Hz}, 3 \mathrm{H}) \mathrm{ppm}$. Major isomer: ${ }^{13} \mathrm{C}$ NMR (151 MHz, $\left.\mathrm{CDCl}_{3}\right): \delta$ 169.0, 138.4, 134.1, 134.0, 133.0, 128.3, 128.3, 124.4, 107.4, 44.1, 26.4, 21.2, 19.0, 18.8, 18.1, 16.5 ppm. Minor isomer: ${ }^{13} \mathrm{C} \mathrm{NMR} \mathrm{(151} \mathrm{MHz,} \mathrm{CDCl}_{3}$ ): $\delta$ 168.6, 138.2, $134.2,133.7,133.1,128.8,128.0,122.2,109.6,48.9,27.9,19.6,19.0,18.2,17.0 \mathrm{ppm}$. One peak could not be detected presumably due to an overlapping with a peak of the major species. IR (neat) $\mathbf{V}_{\text {max }}$ : 2972, 2923, 2850, 1664, 1629, 1411, 1357, 1281, 1039, 1012, 912, 876, 768, 720. HRMS (ESI ${ }^{+}$): exact mass calculated for $[\mathrm{M}+\mathrm{H}]^{+}\left(\mathrm{C}_{16} \mathrm{H}_{22} \mathrm{NO}^{+}\right)$requires $244.1701 \mathrm{~m} / \mathrm{z}$, found $244.1695 \mathrm{~m} / \mathrm{z}$.

\section{Mesityl(2-phenyl-2,3-dihydro-1H-pyrrol-1-yl)methanone (2m)}

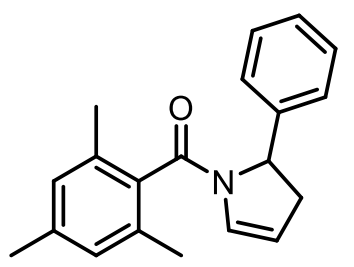

Following GP3 yielded the title compound $(41.1 \mathrm{mg}, 47 \%)$ as colorless oil. The desired enamide was obtained as two rotamers in a ratio of around 5.5:1. Both rotamers: ${ }^{1} \mathrm{H}$ NMR $\left(400 \mathrm{MHz}, \mathrm{CDCl}_{3}\right): \delta 7.50-7.10(\mathrm{~m}, 5 \mathrm{H}), 6.87-6.83(\mathrm{~m}$, 1.7H), $6.78-6.75(\mathrm{~m}, 0.3 \mathrm{H}), 6.50($ app s, $0.2 \mathrm{H}), 6.08(\mathrm{app} \mathrm{s}, 0.8 \mathrm{H}), 5.57$ (dd, $J=$ $10.8,3.7 \mathrm{~Hz}, 0.8 \mathrm{H}), 5.42(\mathrm{~s}, 0.2 \mathrm{H}), 5.18-5.12(\mathrm{~m}, 0.8 \mathrm{H}), 4.58(\mathrm{dd}, J=10.4,3.1$ $\mathrm{Hz}, 0.2 \mathrm{H}), 3.41-3.28(\mathrm{~m}, 1 \mathrm{H}), 2.71-2.60(\mathrm{~m}, 1 \mathrm{H}), 2.32-2.24(\mathrm{~m}, 6 \mathrm{H}), 2.09(\mathrm{~s}$, 2.5H), 1.37 (s, 0.5H) ppm. Major isomer: $\left.{ }^{13} \mathrm{C} \mathrm{NMR} \mathrm{(101} \mathrm{MHz,} \mathrm{CDCl}\right)$ : $\delta 167.3,143.5,138.5,134.4,134.1$, $133.6,130.5,128.8,128.5,128.3,127.5,126.1,110.0,59.8,39.7,21.2,19.2,18.9$ ppm. Minor isomer: ${ }^{13} \mathrm{C}$ NMR could not be clearly identified. IR (neat) $\mathbf{v}_{\text {max }}$ : 3030, 2920, 2858, 1642, 1610, 1389, 1341, 829, 756, 698. HRMS (ESI $\left.{ }^{+}\right)$: exact mass calculated for $[\mathrm{M}+\mathrm{H}]^{+}\left(\mathrm{C}_{20} \mathrm{H}_{22} \mathrm{NO}^{+}\right)$requires $\mathrm{m} / \mathrm{z} 292.1696$, found $\mathrm{m} / \mathrm{z} 292.1694$.

\section{((4aS,8aR)-4a,5,6,7,8,8a-Hexahydroquinolin-1(4H)-yl)(mesityl)methanone (2n)}<smiles>Cc1cc(C)c(C(=O)N2C=CC[C@H]3CCCC[C@H]32)c(C)c1</smiles>

Following GP3 yielded the title compound as white solid (34.5 mg, 41\%). No rotameric effects were observed via NMR spectroscopy. ${ }^{1} \mathrm{H}$ NMR $(600 \mathrm{MHz}$, $\left.\mathrm{CDCl}_{3}\right): \delta 6.82(\mathrm{~m}, 2 \mathrm{H}), 6.01(\mathrm{dd}, J=8.0,2.9 \mathrm{~Hz}, 1 \mathrm{H}), 4.94-4.90(\mathrm{~m}, 1 \mathrm{H}), 3.56$ $(\mathrm{td}, J=10.7,2.9 \mathrm{~Hz}, 1 \mathrm{H}), 3.29-3.24(\mathrm{~m}, 1 \mathrm{H}), 2.27(\mathrm{~s}, 3 \mathrm{H}), 2.21(\mathrm{~s}, 3 \mathrm{H}), 2.13$ $(\mathrm{s}, 3 \mathrm{H}), 1.93-1.87(\mathrm{~m}, 1 \mathrm{H}), 1.86-1.81(\mathrm{~m}, 2 \mathrm{H}), 1.79-1.71(\mathrm{~m}, 2 \mathrm{H}), 1.61-$ $1.50(\mathrm{~m}, 2 \mathrm{H}), 1.41-1.31(\mathrm{~m}, 1 \mathrm{H}), 1.29-1.22(\mathrm{~m}, 1 \mathrm{H}), 1.17-1.10(\mathrm{~m}, 1 \mathrm{H}) \mathrm{ppm} .{ }^{13} \mathrm{C}$ NMR (151 MHz, $\left.\mathrm{CDCl}_{3}\right): \delta 170.8,138.2,134.5,134.4,133.1,128.4,128.3,126.9,109.3,60.4,41.9,33.4,31.5,28.3,26.5$, 25.5, 21.2, 19.2, 18.8 ppm. IR (neat) $\mathbf{v}_{\text {max }}$ : 2922, 2855, 1666, 1633, 1446, 1394, 1368, 1350, 1331, 1306, $1268,1245,1107,1001,850,723$. HRMS (ESI $\left.{ }^{+}\right)$: exact mass calculated for $[\mathrm{M}+\mathrm{H}]^{+}\left(\mathrm{C}_{19} \mathrm{H}_{26} \mathrm{NO}^{+}\right)$requires $\mathrm{m} / \mathrm{z} 284.2009$ found $\mathrm{m} / \mathrm{z} 284.2003$.

\section{$\mathrm{N}$-Cyclohexyl-2,4,6-trimethyl-N-vinylbenzamide (20)}<smiles>C=CN(C(=O)c1c(C)cc(C)cc1C)C1CCCCC1</smiles>

Following GP3 yielded the title compound ( $44.2 \mathrm{mg}, 54 \%$ ) as white solid. The desired enamide was obtained as two rotamers in a ratio of around 20:1. Only the major isomer is described: ${ }^{1} \mathrm{H}$ NMR $\left(600 \mathrm{MHz}, \mathrm{CDCl}_{3}\right): \delta 6.82(\mathrm{~s}, 2 \mathrm{H})$, $6.18(\mathrm{dd}, J=15.8,9.3 \mathrm{~Hz}, 1 \mathrm{H}), 4.63(\mathrm{dd}, J=15.8,0.7 \mathrm{~Hz}, 1 \mathrm{H}), 4.31-4.28(\mathrm{~m}$, $2 \mathrm{H}), 2.27(\mathrm{~s}, 3 \mathrm{H}), 2.25-2.18(\mathrm{~m}, 2 \mathrm{H}), 2.16(\mathrm{~s}, 6 \mathrm{H}), 1.89-1.85(\mathrm{~m}, 2 \mathrm{H}), 1.81-$ $1.74(\mathrm{~m}, 2 \mathrm{H}), 1.72-1.65(\mathrm{~m}, 1 \mathrm{H}), 1.44-1.36(\mathrm{~m}, 2 \mathrm{H}), 1.27-1.20(\mathrm{~m}, 1 \mathrm{H}) \mathrm{ppm} .{ }^{13} \mathrm{C}$ NMR (101 MHz, $\mathrm{CDCl}_{3}$ ): $\delta 170.9,138.1,134.3,133.4,133.2,128.2,98.4,54.9,29.2,26.4,25.5,21.1,18.8$ ppm. IR (neat) 
$\mathbf{V}_{\text {max }}$ : 2930, 2856, 1659, 1611, 1435, 1342, 1316, 1218, 851, 729. HRMS (ESI ${ }^{+}$: exact mass calculated for $[\mathrm{M}+\mathrm{H}]^{+}\left(\mathrm{C}_{18} \mathrm{H}_{26} \mathrm{NO}^{+}\right)$requires $\mathrm{m} / \mathrm{z} 272.2014$, found $\mathrm{m} / \mathrm{z} 272.2010$.

N-Butyl-2,4,6-trimethyl-N-vinylbenzamide

$+(E)-N$-(but-1-en-1-yl)-N-ethyl-2,4,6-trimethylbenzamide (2p, 2p')<smiles>C=CN(CCCC)C(=O)c1c(C)cc(C)cc1C</smiles>

A
B

Following GP3 yielded the two title compounds $(36.9 \mathrm{mg}, 50 \%)$ as colorless oils in a ratio $\mathrm{A}: \mathrm{B}$ of 1.8:1. Small amounts of each compound was possible to be isolated separately and was used for the full characterization.

Product A (2p): Via NMR spectroscopy two rotamers were observed in a ratio of around 12:1. Only the major isomer is described: ${ }^{1} \mathbf{H}$ NMR (600 $\left.\mathrm{MHz}, \mathrm{CDCl}_{3}\right): \delta 6.85(\mathrm{~s}, 2 \mathrm{H}), 6.32(\mathrm{dd}, J=15.6,9.3 \mathrm{~Hz}, 1 \mathrm{H}), 4.47(\mathrm{~d}, J=15.6 \mathrm{~Hz}, 1 \mathrm{H}), 4.16(\mathrm{~d}, J=9.3 \mathrm{~Hz}$, $1 \mathrm{H}), 3.83-3.77(\mathrm{~m}, 2 \mathrm{H}), 2.28(\mathrm{~s}, 3 \mathrm{H}), 2.15(\mathrm{~s}, 6 \mathrm{H}), 1.70-1.64(\mathrm{~m}, 2 \mathrm{H}), 1.46-1.41(\mathrm{~m}, 2 \mathrm{H}), 0.99(\mathrm{t}, J=$ $7.4 \mathrm{~Hz}, 3 \mathrm{H}$ ) ppm. ${ }^{13} \mathrm{C} \mathrm{NMR}\left(151 \mathrm{MHz}, \mathrm{CDCl}_{3}\right): \delta 170.8,138.6,134.0,133.5,133.1,128.4,93.3,41.2,28.7$, 21.3, 20.6, 19.014 .0 ppm. IR (neat) $\mathbf{v}_{\text {max }}$ : 2958, 2929, 2872, 1666, 1621, 1402, 1379, 1356, 1312, 1218, 1090, 851. HRMS $\left(\mathrm{ESI}^{+}\right)$: exact mass calculated for $[\mathrm{M}+\mathrm{H}]^{+}\left(\mathrm{C}_{16} \mathrm{H}_{24} \mathrm{NO}^{+}\right)$requires $\mathrm{m} / \mathrm{z} 246.1852$ found $\mathrm{m} / \mathrm{z} 246.1846$.

Product B (2p'): Via NMR spectroscopy two rotamers were observed in a ratio of around 5:1. Both rotamers are described together: ${ }^{1} \mathbf{H}$ NMR $\left(400 \mathrm{MHz}, \mathrm{CDCl}_{3}\right): \delta 7.37(\mathrm{dd}, J=14.5,4.8 \mathrm{~Hz}, 0.2 \mathrm{H}), 6.84(\mathrm{~s}$, $2 \mathrm{H}), 6.03(\mathrm{~d}, J=14.1 \mathrm{~Hz}, 0.8 \mathrm{H}), 5.22-5.14(\mathrm{~m}, 0.2 \mathrm{H}), 5.07(\mathrm{dt}, J=14.0,6.9 \mathrm{~Hz}, 0.8 \mathrm{H}), 3.87(\mathrm{q}, J=7.1$ $\mathrm{Hz}, 2 \mathrm{H}), 2.29(\mathrm{~s}, 3 \mathrm{H}), 2.15(\mathrm{~s}, 6 \mathrm{H}), 1.89$ (quint, $J=6.3 \mathrm{~Hz}, 2 \mathrm{H}), 1.28-1.23(\mathrm{~m}, 3 \mathrm{H}), 0.89(\mathrm{t}, J=7.4 \mathrm{~Hz}, 3 \mathrm{H}$ ) ppm. ${ }^{13} \mathrm{C}$ NMR (151 MHz, $\mathrm{CDCl}_{3}$, major rotamer): $\delta$ 170.0, 138.4, 133.9, 133.4, 128.4, 126.3, 113.1, 36.9, 23.7, 21.3, 19.0, 15.0, $12.1 \mathrm{ppm}$. Minor isomer: ${ }^{13} \mathrm{C}$ NMR could not be clearly identified. IR (neat) $\mathbf{V}_{\text {max: }}$ 2961, 2922, 1641, 1612, 1451, 1396, 1323, 1082. HRMS (ESI $\left.{ }^{+}\right)$: exact mass calculated for [M+H] $]^{+}$ $\left(\mathrm{C}_{16} \mathrm{H}_{24} \mathrm{NO}^{+}\right)$requires $\mathrm{m} / \mathrm{z} 246.1852$ found $\mathrm{m} / \mathrm{z} 246.1846$.

((3S,4R)-3-((Benzo[d][1,3]dioxol-5-yloxy)methyl)-4-(4-fluorophenyl)-3,4-dihydropyridin-1(2H)yl)(mesityl)methanone (2q)<smiles>Cc1cc(C)c(C(=O)N2C=C[C@H](c3ccc(F)cc3)[C@H](COc3ccc4c(c3)OCO4)C2)c(C)c1</smiles>

Following GP3 yielded title compound ( $37.2 \mathrm{mg}, 28 \%$ ) as colorless oil. The desired enamide was obtained as two rotamers in a ratio of around 4:1. Both rotamers are described together: ${ }^{1} \mathbf{H}$ NMR (600 $\left.\mathrm{MHz}, \mathrm{CDCl}_{3}\right): \delta 7.61(\mathrm{dd}, J=8.4,1.9 \mathrm{~Hz}, 0.2 \mathrm{H}), 7.18-7.12(\mathrm{~m}, 2 \mathrm{H})$, $7.03-6.97(\mathrm{~m}, 2 \mathrm{H}), 6.90-6.84(\mathrm{~m}, 2 \mathrm{H}), 6.69(\mathrm{~d}, J=8.4 \mathrm{~Hz}, 0.8 \mathrm{H})$, $6.64(\mathrm{~d}, J=8.4 \mathrm{~Hz}, 0.2 \mathrm{H}), 6.47(\mathrm{~d}, J=2.4 \mathrm{~Hz}, 0.8 \mathrm{H}), 6.32-6.26(\mathrm{~m}$, $1.7 \mathrm{H}), 6.11(\mathrm{dd}, J=8.5,2.5 \mathrm{~Hz}, 0.3 \mathrm{H}), 5.92-5.90(\mathrm{~m}, 2 \mathrm{H}), 5.20(\mathrm{dd}$, $J=8.4,3.3 \mathrm{~Hz}, 0.2 \mathrm{H}), 4.80(\mathrm{dd}, J=8.3,3.0 \mathrm{~Hz}, 0.8 \mathrm{H}), 4.40(\mathrm{dd}, J=$ 13.4, 3.6 Hz, $0.8 \mathrm{H}), 3.87(\mathrm{dd}, J=9.3,4.4 \mathrm{~Hz}, 0.7 \mathrm{H}), 3.79-3.71(\mathrm{~m}$, $1.7 \mathrm{H}), 3.66-3.59(\mathrm{~m}, 1 \mathrm{H}), 3.51-3.49(\mathrm{~m}, 0.3 \mathrm{H}), 3.42(\mathrm{dd}, J=12.8$, $3.2 \mathrm{~Hz}, 0.2 \mathrm{H}), 3.30(\mathrm{dd}, J=12.8,8.4 \mathrm{~Hz}, 0.2 \mathrm{H}), 2.33-2.25(\mathrm{~m}, 6 \mathrm{H}), 2.20-2.16(\mathrm{~m}, 4 \mathrm{H}) \mathrm{ppm} .{ }^{13} \mathrm{C}$ NMR

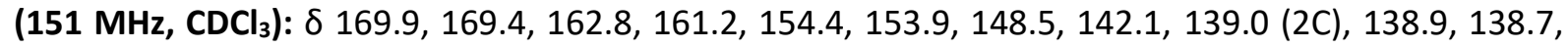
$138.1,134.2,134.1,133.9,133.7,132.8,132.6,129.7$ (2C), 128.9, 128.6, 128.5, 128.3, 126.3, 124.5, 115.7, 115.6, 111.9, 110.6, 108.1, 108.0, 105.8, 105.4, 101.3, 98.3, 98.0, 68.7, 68.1, 44.7, 41.4, 41.1, 40.7, 40.6, 40.4, 21.3 (2C), 19.2 (2C), 19.1 (2C) ppm. ${ }^{19} \mathrm{~F} \mathrm{NMR} \mathrm{(376} \mathrm{MHz,} \mathrm{CDCl} 3$ ): $\delta$-116.1, -116.2 ppm. IR (neat) $\mathbf{v}_{\text {max }}$ : 1632, 1505, 1487, 1469, 1183, 1037, 732. HRMS (ESI ${ }^{+}$): exact mass calculated for $[\mathrm{M}+\mathrm{Na}]^{+}\left(\mathrm{C}_{29} \mathrm{H}_{28} \mathrm{FNO}_{4} \mathrm{Na}^{+}\right)$requires $496.1900 \mathrm{~m} / \mathrm{z}$ found $496.1881 \mathrm{~m} / \mathrm{z}$. 
<smiles>COc1ccc(CC2c3cc(OC)c(OC)cc3C=CN2C(=O)c2c(C)cc(C)cc2C)cc1OC</smiles>

Following GP3 using $\mathrm{Et}_{2} \mathrm{O} / \mathrm{DCM}(3.5 \mathrm{~mL}, 5: 1)$ yielded the title compound ( $80.9 \mathrm{mg}, 55 \%)$ as white solid. The desired enamide was obtained as two rotamers in a ratio of around 13:1. Only the major isomer is described: ${ }^{1} \mathrm{H}$ NMR $\left(600 \mathrm{MHz}, \mathrm{CDCl}_{3}\right): \delta 6.89(\mathrm{~s}, 1 \mathrm{H}), 6.83$ $(\mathrm{s}, 1 \mathrm{H}), 6.70(\mathrm{~d}, J=8.1 \mathrm{~Hz}, 1 \mathrm{H}), 6.65(\mathrm{~d}, J=1.6 \mathrm{~Hz}, 1 \mathrm{H}), 6.58(\mathrm{~s}, 1 \mathrm{H})$, $6.48(\mathrm{dd}, J=8.1,1.6 \mathrm{~Hz}, 1 \mathrm{H}), 6.04(\mathrm{~d}, J=7.8 \mathrm{~Hz}, 1 \mathrm{H}), 5.95-5.91(\mathrm{~m}$, $2 \mathrm{H}), 5.68(\mathrm{~d}, J=7.7 \mathrm{~Hz}, 1 \mathrm{H}), 3.85(\mathrm{~s}, 3 \mathrm{H}), 3.83(\mathrm{~s}, 3 \mathrm{H}), 3.80(\mathrm{~s}, 3 \mathrm{H})$, $3.53(\mathrm{~s}, 3 \mathrm{H}), 3.03(\mathrm{dd}, J=12.6,4.9 \mathrm{~Hz}, 1 \mathrm{H}), 2.88(\mathrm{dd}, J=12.5,9.7 \mathrm{~Hz}$, $1 \mathrm{H}), 2.29(\mathrm{~s}, 3 \mathrm{H}), 2.23(\mathrm{~s}, 3 \mathrm{H}), 2.03(\mathrm{~s}, 3 \mathrm{H}) \mathrm{ppm} .{ }^{13} \mathrm{C} \mathrm{NMR}(151 \mathrm{MHz}$, $\left.\mathrm{CDCl}_{3}\right): \delta 169.8,148.8,148.3,147.9,147.3,138.9,134.6,134.2,132.5,130.1,128.5,128.4,124.3$, $123.2,122.8,122.6,113.3$ (2C), 111.0, 110.1, 108.1, 56.1, 56.0 (2C), 55.8, 55.2, 40.4, 21.3, 18.9 (2C) ppm. IR (neat) $\mathbf{v}_{\text {max }}$ : 2936, 1623, 1512, 1425, 1341, 1265, 1231, 1138, 1127, 1102, 912, 883, 728. HRMS $\left(\mathrm{ESI}^{+}\right)$: exact mass calculated for $[\mathrm{M}+\mathrm{H}]^{+}\left(\mathrm{C}_{30} \mathrm{H}_{34} \mathrm{NO}_{5}{ }^{+}\right)$requires $\mathrm{m} / \mathrm{z} 488.2431$ found $\mathrm{m} / \mathrm{z} 488.2428$.

\section{(2,3-Dihydro-1H-pyrrol-1-yl)(2,4,6-triisopropylphenyl)methanone (4a)}<smiles>CC(C)c1cc(C(C)C)c(C(=O)N2C=CCC2)c(C(C)C)c1</smiles>

Following GP3 yielded the title compound $(62.6 \mathrm{mg}, 70 \%)$ as colorless oil. The desired enamide was obtained as two rotamers in a ratio of 7.1:1. Major isomer: $\left.{ }^{1} \mathbf{H ~ N M R ~ ( 7 0 0 ~ M H z , ~} \mathrm{CDCl}_{3}\right): \delta 6.99(\mathrm{~s}, 2 \mathrm{H}), 5.97-5.93(\mathrm{~m}, 1 \mathrm{H}), 5.09-$ $5.06(\mathrm{~m}, 1 \mathrm{H}), 4.04(\mathrm{t}, J=8.8 \mathrm{~Hz}, 2 \mathrm{H}), 2.97-2.65(\mathrm{~m}, 5 \mathrm{H}), 1.23(\mathrm{~d}, J=6.8 \mathrm{~Hz}$, $12 \mathrm{H}), 1.16(\mathrm{~d}, J=6.8 \mathrm{~Hz}, 6 \mathrm{H}) \mathrm{ppm}$. Minor isomer: ${ }^{1} \mathrm{H}$ NMR $\left(700 \mathrm{MHz}, \mathrm{CDCl}_{3}\right): \delta 7.16-7.13(\mathrm{~m}, 1 \mathrm{H}), 6.99$ $(\mathrm{s}, 2 \mathrm{H}), 5.35-5.31(\mathrm{~m}, 1 \mathrm{H}), 3.41(\mathrm{t}, J=8.6 \mathrm{~Hz}, 2 \mathrm{H}), 2.97-2.65(\mathrm{~m}, 5 \mathrm{H}), 1.23(\mathrm{~d}, J=6.8 \mathrm{~Hz}, 12 \mathrm{H}), 1.16$ $(\mathrm{d}, J=6.8 \mathrm{~Hz}, 6 \mathrm{H})$ ppm. Major isomer: ${ }^{13} \mathrm{C}$ NMR $\left(176 \mathrm{MHz}, \mathrm{CDCl}_{3}\right): \delta 167.7,149.9,144.7,132.3,130.7$, $121.3,111.2,44.4,34.5,31.3,28.9,24.9,24.1,23.9 \mathrm{ppm}$. Minor isomer: ${ }^{13} \mathrm{C}$ NMR could not be clearly identified. IR (neat) $\mathbf{v}_{\text {max }}$ : 2959, 2928, 2868, 1640, 1611, 1460, 1408, 1384, 1363, 756. HRMS (ESI ${ }^{+}$): exact mass calculated for $[\mathrm{M}+\mathrm{Na}]^{+}\left(\mathrm{C}_{20} \mathrm{H}_{29} \mathrm{NNaO}^{+}\right)$requires $\mathrm{m} / \mathrm{z} 322.2141$, found $\mathrm{m} / \mathrm{z} 322.2139$.

\section{Anthracen-9-yl(2,3-dihydro-1H-pyrrol-1-yl)methanone (4b)}<smiles>O=C(c1c2ccccc2cc2ccccc12)N1C=CCC1</smiles>

Following GP3 yielded the title compound ( $47.9 \mathrm{mg}, 58 \%$ ) as pale yellow solid. The desired enamide was obtained as two rotamers in a ratio of 5.3:1. Major isomer: ${ }^{1} \mathrm{H} \mathrm{NMR}\left(600 \mathrm{MHz}, \mathrm{CDCl}_{3}\right): \delta 8.50(\mathrm{~s}, 1 \mathrm{H}), 8.03(\mathrm{~d}, J=7.7 \mathrm{~Hz}, 2 \mathrm{H}), 7.90(\mathrm{~d}$, $J=8.2 \mathrm{~Hz}, 2 \mathrm{H}), 7.56-7.47(\mathrm{~m}, 4 \mathrm{H}), 5.63(\mathrm{dt}, J=4.3,2.2 \mathrm{~Hz}, 1 \mathrm{H}), 5.05(\mathrm{dt}, J=4.9$, $2.6 \mathrm{~Hz}, 1 \mathrm{H}), 4.40-4.31(\mathrm{~m}, 2 \mathrm{H}), 2.87-2.80(\mathrm{~m}, 2 \mathrm{H}) \mathrm{ppm}$. Minor isomer: ${ }^{1} \mathrm{H}$ NMR $\left(600 \mathrm{MHz}, \mathrm{CDCl}_{3}\right): \delta 8.50(\mathrm{~s}, 1 \mathrm{H}), 8.03(\mathrm{~d}, J=7.7 \mathrm{~Hz}, 2 \mathrm{H}), 7.97(\mathrm{~d}, J=8.4 \mathrm{~Hz}, 2 \mathrm{H})$, $7.56-7.47(\mathrm{~m}, 4 \mathrm{H}), 7.44(\mathrm{dt}, J=4.3,2.2 \mathrm{~Hz}, 1 \mathrm{H}), 5.46(\mathrm{dt}, J=4.9,2.6 \mathrm{~Hz}, 1 \mathrm{H}), 3.33-3.26(\mathrm{~m}, 2 \mathrm{H}), 2.66$ -2.56 (m, 2H) ppm. $\left.{ }^{13} \mathrm{C} \mathrm{NMR} \mathrm{(151} \mathrm{MHz,} \mathrm{CDCl} 3\right): \delta 166.2,165.8,131.4,131.3,130.7,130.2,129.1,128.9$, $128.8,128.4,128.2,128.1,127.5,127.2,127.1,125.7,125.2,124.9,113.0,112.6,46.4,45.0,30.0$ (2C) ppm. Minor isomer: ${ }^{13} \mathrm{C}$ NMR could not be clearly identified. IR (neat) $\mathbf{v}_{\text {max }}: 1632,1611,1435,1400$, 1328, 1276, 749. HRMS (ESI $\left.{ }^{+}\right)$: exact mass calculated for $[\mathrm{M}+\mathrm{Na}]^{+}\left(\mathrm{C}_{19} \mathrm{H}_{15} \mathrm{NNaO}^{+}\right)$requires $\mathrm{m} / \mathrm{z}$ 296.1046, found $\mathrm{m} / \mathrm{z} 296.1038$.

\section{(2,3-Dihydro-1H-pyrrol-1-yl)(phenyl)methanone (4c)}

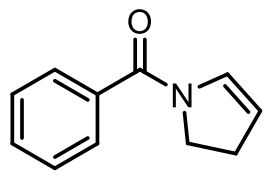

Following GP3 yielded the title compound ( $28.8 \mathrm{mg}, 55 \%)$ as orange oil. The desired enamide was obtained as two rotamers in a ratio of 6.4:1. Major isomer: ${ }^{1} \mathbf{H}$ NMR $\left(400 \mathrm{MHz}, \mathrm{CDCl}_{3}\right): \delta 7.55-7.38(\mathrm{~m}, 5 \mathrm{H}), 6.47-6.44(\mathrm{~m}, 1 \mathrm{H}), 5.18$ (app s, $\left.1 \mathrm{H}\right), 4.03$ $(\mathrm{t}, J=8.8 \mathrm{~Hz}, 2 \mathrm{H}), 2.76-2.66(\mathrm{~m}, 2 \mathrm{H}) \mathrm{ppm}$. Minor isomer: ${ }^{1} \mathrm{H} \mathrm{NMR}\left(400 \mathrm{MHz}, \mathrm{CDCl}_{3}\right)$ : $\delta 7.55-7.38(\mathrm{~m}, 5 \mathrm{H}), 7.10(\mathrm{app} \mathrm{s}, 1 \mathrm{H}), 5.37$ (app s, 1H), 3.86- $3.76(\mathrm{~m}, 2 \mathrm{H}), 2.76-2.66(\mathrm{~m}, 2 \mathrm{H}) \mathrm{ppm}$. Major isomer: ${ }^{13} \mathrm{C} \mathrm{NMR} \mathrm{(176} \mathrm{MHz,} \mathrm{CDCl} 3$ ): $\delta$ 167.1, 136.0, 130.8, 130.4, 128.6, 127.8, 111.8, 45.8, 28.5 ppm. Minor isomer: ${ }^{13} \mathrm{C}$ NMR could not be clearly identified. IR (neat) $\mathbf{v}_{\max }: 2959,2925,1576,1446$, 
1367, 830, 700. HRMS (ESI $\left.{ }^{+}\right)$: exact mass calculated for $[\mathrm{M}+\mathrm{H}]^{+}\left(\mathrm{C}_{11} \mathrm{H}_{12} \mathrm{NO}^{+}\right)$requires $174.0919 \mathrm{~m} / \mathrm{z}$, found $174.0912 \mathrm{~m} / \mathrm{z}$.

\section{(3,4-Dihydropyridin-1(2H)-yl)(phenyl)methanone (4d)}<smiles>O=C(c1ccccc1)N1C=CCCC1</smiles>

Following GP3 yielded the title compound (13.5 mg, 24\%) as colorless oil. The desired enamide was obtained as two rotamers in a ratio of 3.4:1. Major isomer: ${ }^{1} \mathrm{H}$ NMR $\left(600 \mathrm{MHz}, \mathrm{CDCl}_{3}\right): \delta 7.49-7.37(\mathrm{~m}, 5 \mathrm{H}), 6.45(\mathrm{~d}, J=8.1 \mathrm{~Hz}, 1 \mathrm{H}), 4.90-$ $4.80(\mathrm{~m}, 1 \mathrm{H}), 3.89-3.78(\mathrm{~m}, 2 \mathrm{H}), 2.12(\mathrm{tdd}, J=6.1,3.9,2.0 \mathrm{~Hz}, 2 \mathrm{H}), 1.98-1.92(\mathrm{~m}$, 2H) ppm. Minor isomer: ${ }^{1} \mathrm{H} \mathrm{NMR}\left(600 \mathrm{MHz}, \mathrm{CDCl}_{3}\right): \delta 7.49-7.37(\mathrm{~m}, 5 \mathrm{H}), 7.35-7.27(\mathrm{~m}, 1 \mathrm{H}), 5.29$ (app s, 1H), 3.54 (app s, 1H), 2.12 (tdd, J = 6.1, 3.9, $2.0 \mathrm{~Hz}, 2 \mathrm{H}), 1.79(\mathrm{br}, 2 \mathrm{H})$ ppm. Major isomer: ${ }^{13} \mathrm{C}$ NMR (151 MHz, $\mathrm{CDCl}_{3}$ ): $\delta$ 169.5, 135.3, 130.3, 128.5, 128.3, 127.7, 107.7, 41.23, 22.03, 21.81 ppm. Minor isomer: ${ }^{13} \mathrm{C}$ NMR could not be clearly identified. All spectral data were found in accordance to the literature. ${ }^{[17]}$

\section{(3,4-Dihydropyridin-1(2H)-yl)(o-tolyl)methanone (4e)}<smiles>Cc1ccccc1C(=O)N1C=CCCC1</smiles>

Following GP3 yielded the title compound ( $38.9 \mathrm{mg}, 64 \%$ ) as yellow oil. The desired enamide was obtained as two rotamers in a ratio of 3.4:1. Major isomer: ${ }^{1} \mathbf{H}$ NMR (400 MHz, $\left.\mathrm{CDCl}_{3}\right): \delta 7.28(\mathrm{~m}, 1 \mathrm{H}), 7.24-7.14(\mathrm{~m}, 3 \mathrm{H}), 6.18-6.10(\mathrm{~m}, 1 \mathrm{H}), 4.90-$ $4.81(\mathrm{~m}, 1 \mathrm{H}), 3.90$ (app s, 2H), $2.29(\mathrm{~s}, 3 \mathrm{H}), 2.14-2.07(\mathrm{~m}, 2 \mathrm{H}), 1.91-1.80(\mathrm{~m}, 2 \mathrm{H})$ ppm. Minor isomer: ${ }^{1} \mathbf{H}$ NMR $\left(400 \mathrm{MHz}, \mathrm{CDCl}_{3}\right): \delta 7.38(\mathrm{~d}, J=8.3 \mathrm{~Hz}, 1 \mathrm{H}), 7.35-7.25(\mathrm{~m}, 1 \mathrm{H}), 7.24-$ $7.14(\mathrm{~m}, 3 \mathrm{H}), 5.28-5.20(\mathrm{~m}, 1 \mathrm{H}), 3.32-3.28(\mathrm{~m}, 2 \mathrm{H}), 2.31(\mathrm{~s}, 3 \mathrm{H}), 2.14-2.07(\mathrm{~m}, 2 \mathrm{H}), 1.78(\mathrm{~s}, 2 \mathrm{H})$ ppm. Major isomer: $\left.{ }^{13} \mathrm{C} \mathrm{NMR} \mathrm{(101} \mathrm{MHz,} \mathrm{CDCl}\right)$ ): $\delta 169.3,135.6,134.6,130.3,129.2,126.6,126.4,125.9$, 108.1, 40.4, 21.9, 21.5, 19.0 ppm. Minor isomer: $\left.{ }^{13} \mathrm{C} \mathrm{NMR} \mathrm{(101} \mathrm{MHz,} \mathrm{CDCl}_{3}\right): \delta 168.5,135.9,134.3$, 130.3, 129.0, 125.9, 125.8, 124.0, 110.5, 45.3, 22.3, 22.1, 18.9 ppm. IR (neat) $v_{\text {max }}$ : 2926, 1630, 1373, 1355, 1321, 1257, 992, 739, 730, 718, 621. HRMS (ESI $)$ : exact mass calculated for $[\mathrm{M}+\mathrm{H}]^{+}\left(\mathrm{C}_{13} \mathrm{H}_{16} \mathrm{NO}^{+}\right)$ requires $202.1232 \mathrm{~m} / \mathrm{z}$, found $202.1226 \mathrm{~m} / \mathrm{z}$.

\section{(3,4-Dihydropyridin-1(2H)-yl)(p-tolyl)methanone (4f)}<smiles>Cc1ccc(C(=O)N2C=CCCC2)cc1</smiles>

Following GP3 yielded the title compound (20.9 mg, 35\%) as colorless oil. The desired enamide was obtained as two rotamers in a ratio of 3.7:1. Major isomer: ${ }^{1} \mathrm{H}$ NMR $\left(600 \mathrm{MHz}, \mathrm{CDCl}_{3}\right): \delta 7.38(\mathrm{~d}, J=7.7 \mathrm{~Hz}, 2 \mathrm{H}), 7.21(\mathrm{~d}, J=7.9 \mathrm{~Hz}, 2 \mathrm{H}), 6.48$ (d, J = 7.2 Hz, 1H), 4.83 (app s, 1H), 3.82 (app s, 2H), $2.38(\mathrm{~s}, 2 \mathrm{H}), 2.15-2.09$ (m, 2H), 1.95 (app s, 2H) ppm. Minor isomer: ${ }^{1} \mathrm{H}$ NMR (600 MHz, $\left.\mathrm{CDCl}_{3}\right): \delta 7.38$ (d, J $\left.=7.7 \mathrm{~Hz}, 2 \mathrm{H}\right), 7.21$ (d, $J=7.9 \mathrm{~Hz}, 2 \mathrm{H}), 5.21(\mathrm{~s}, 1 \mathrm{H}), 3.58(\mathrm{~s}, 2 \mathrm{H}), 2.38(\mathrm{~s}, 3 \mathrm{H}), 2.15-2.09(\mathrm{~m}, 3 \mathrm{H}), 1.79(\mathrm{~s}, 2 \mathrm{H}) \mathrm{ppm}$. One peak was not detected, due to an overlapping with another signal. Major isomer: $\left.{ }^{13} \mathrm{C} \mathrm{NMR} \mathrm{(151} \mathbf{~ M H z}, \mathrm{CDCl}_{3}\right)$ : $\delta 169.6,140.6,132.3,129.1,128.5,127.9,107.3,41.3,22.1,21.9,21.6 \mathrm{ppm}$. Minor isomer: ${ }^{13} \mathrm{C}$ NMR could not be clearly identified. IR (neat) $\mathbf{v}_{\text {max }}$ : 2925, 1632, 1407, 1375, 1356, 1291, 1258, 994, 753, 579. HRMS (ESI $\left.{ }^{+}\right)$: exact mass calculated for $[\mathrm{M}+\mathrm{H}]^{+}\left(\mathrm{C}_{13} \mathrm{H}_{16} \mathrm{NO}^{+}\right)$requires $202.1232 \mathrm{~m} / \mathrm{z}$, found 202.1225 $\mathrm{m} / \mathrm{z}$.

\section{(3,4-Dihydropyridin-1(2H)-yl)(2-methoxyphenyl)methanone (4g)}<smiles>COc1ccccc1C(=O)N1C=CCCC1</smiles>

Following GP3 yielded the title compound $(29.9 \mathrm{mg}, 46 \%)$ as yellowish oil. The desired enamide was obtained as two rotamers in a ratio of 2.3:1. Major isomer: ${ }^{1} \mathrm{H}$ NMR $\left(600 \mathrm{MHz}, \mathrm{CDCl}_{3}\right): \delta 7.39-7.35(\mathrm{~m}, 1 \mathrm{H}), 7.22(\mathrm{dd}, J=7.4,1.7 \mathrm{~Hz}, 1 \mathrm{H}), 7.00$ $-6.96(\mathrm{~m}, 1 \mathrm{H}), 6.92(\mathrm{~d}, J=8.3 \mathrm{~Hz}, 1 \mathrm{H}), 6.19(\mathrm{dt}, J=8.4,1.9 \mathrm{~Hz}, 1 \mathrm{H}), 4.79(\mathrm{dt}, J=8.1$, $3.9 \mathrm{~Hz}, 1 \mathrm{H}), 3.90-3.85(\mathrm{~m}, 2 \mathrm{H}), 3.81(\mathrm{~s}, 3 \mathrm{H}), 2.14-2.05(\mathrm{~m}, 2 \mathrm{H}), 1.95-1.87(\mathrm{~m}, 2 \mathrm{H}) \mathrm{ppm}$. Minor isomer: ${ }^{1} \mathrm{H}$ NMR $\left(600 \mathrm{MHz}, \mathrm{CDCl}_{3}\right): \delta 7.38-7.33(\mathrm{~m}, 2 \mathrm{H}), 7.28-7.26(\mathrm{~m}, 1 \mathrm{H}), 7.02-6.98(\mathrm{~m}, 1 \mathrm{H}), 6.91$ 
$(\mathrm{d}, J=8.3 \mathrm{~Hz}, 1 \mathrm{H}), 5.20(\mathrm{dt}, J=8.1,3.9 \mathrm{~Hz}, 1 \mathrm{H}), 3.83(\mathrm{~s}, 3 \mathrm{H}), 3.44-3.29(\mathrm{~m}, 2 \mathrm{H}), 2.14-2.05(\mathrm{~m}, 2 \mathrm{H})$, $1.82-1.73(\mathrm{~m}, 2 \mathrm{H})$ ppm. Major isomer: ${ }^{13} \mathrm{C} \mathrm{NMR}\left(151 \mathrm{MHz}, \mathrm{CDCl}_{3}\right): \delta 167.5,155.8,130.9,128.5,127.1$, 125.3, 120.9, 111.3, 107.5, 55.8, 40.7, 22.1, 21.6 ppm. Minor isomer: ${ }^{13} \mathbf{C} \mathbf{N M R}\left(151 \mathbf{~ M H z}, \mathbf{C D C l}_{3}\right): \delta$ $166.8,155.7,130.8,128.4,125.8,125.3,121.1,111.0,110.2,55.7,45.1,22.3,22.1 \mathrm{ppm}$. IR (neat) $\mathbf{v}_{\text {max: }}$ 2930, 2840, 1629, 1599, 1370, 1356, 1288, 1245, 1109, 992, 751, 716, 618. HRMS (ESI ${ }^{+}$): exact mass calculated for $[\mathrm{M}+\mathrm{H}]^{+}\left(\mathrm{C}_{13} \mathrm{H}_{16} \mathrm{NO}_{2}{ }^{+}\right)$requires $218.1181 \mathrm{~m} / \mathrm{z}$, found $218.1172 \mathrm{~m} / \mathrm{z}$.

\section{(3,4-Dihydropyridin-1(2H)-yl)(3-methoxyphenyl)methanone (4h)}<smiles>COc1cccc(C(=O)N2C=CCCC2)c1</smiles>

Following GP3 yielded the title compound $(38.5 \mathrm{mg}, 59 \%)$ as yellowish oil. The desired enamide was obtained as two rotamers in a ratio of 3.3:1. Major isomer: ${ }^{1} \mathrm{H} \mathrm{NMR}\left(600 \mathrm{MHz}, \mathrm{CDCl}_{3}\right): \delta 7.31(\mathrm{t}, J=7.9 \mathrm{~Hz}, 1 \mathrm{H}), 7.03(\mathrm{~d}, J=7.4 \mathrm{~Hz}, 1 \mathrm{H}), 7.00(\mathrm{~s}$, $1 \mathrm{H}), 6.98-6.94(\mathrm{~m}, 1 \mathrm{H}), 6.45(\mathrm{~d}, J=8.1 \mathrm{~Hz}, 1 \mathrm{H}), 4.90-4.78(\mathrm{~m}, 1 \mathrm{H}), 3.83(\mathrm{~d}, J=8.9$ $\mathrm{Hz}, 5 \mathrm{H}), 2.17-2.06(\mathrm{~m}, 2 \mathrm{H}), 1.97-1.88(\mathrm{~m}, 2 \mathrm{H})$. Minor isomer: ${ }^{1} \mathrm{H}$ NMR $(600 \mathrm{MHz}$, $\left.\mathrm{CDCl}_{3}\right): \delta 7.31(\mathrm{t}, J=7.9 \mathrm{~Hz}, 1 \mathrm{H}), 7.27(\mathrm{br}, 1 \mathrm{H}), 7.03(\mathrm{~d}, J=7.4 \mathrm{~Hz}, 1 \mathrm{H}), 7.00(\mathrm{~s}, 1 \mathrm{H}), 6.98-6.94(\mathrm{~m}, 1 \mathrm{H})$, $5.23(\mathrm{br}, 1 \mathrm{H}), 3.82(\mathrm{~s}, 3 \mathrm{H}), 3.55(\mathrm{br}, 2 \mathrm{H}), 2.17-2.06(\mathrm{~m}, 2 \mathrm{H}), 1.79(\mathrm{br}, 2 \mathrm{H})$. Major isomer: ${ }^{13} \mathrm{C}$ NMR (151 MHz, $\left.\mathrm{CDCl}_{3}\right): \delta 169.21,159.7,136.6,129.5,127.6,120.5,116.3,113.4,107.7,55.5,41.2,22.0,21.8$. Minor isomer: ${ }^{13} \mathrm{C}$ NMR could not be clearly identified. IR (neat) $\mathbf{v}_{\text {max }}$ : 2929, 2861, 1631, 1579, 1406, 1372, 1356, 1287, 1263, 1041, 994, 790, 746, 720, 624. HRMS (ESI ${ }^{+}$): exact mass calculated for $[\mathrm{M}+\mathrm{H}]^{+}$ $\left(\mathrm{C}_{13} \mathrm{H}_{16} \mathrm{NO}_{2}^{+}\right)$requires $218.1181 \mathrm{~m} / \mathrm{z}$, found $218.1173 \mathrm{~m} / \mathrm{z}$.

\section{(3,4-Dihydropyridin-1(2H)-yl)(4-methoxyphenyl)methanone (4i)}<smiles>COc1ccc(C(=O)N2C=CCCC2)cc1</smiles>

Following GP3 yielded the title compound $(42.5 \mathrm{mg}, 65 \%)$ as yellowish oil. No rotameric effects were observed via NMR spectroscopy. ${ }^{1} \mathbf{H}$ NMR (400 $\left.\mathrm{MHz}, \mathrm{CDCl}_{3}\right) \delta 7.47(\mathrm{~d}, J=8.7 \mathrm{~Hz}, 2 \mathrm{H}), 6.91(\mathrm{~d}, J=8.7 \mathrm{~Hz}, 2 \mathrm{H}), 6.52$ (app s, $1 \mathrm{H}), 4.84($ app s, 1H), $3.86-3.74(\mathrm{~m}, 5 \mathrm{H}), 2.16-2.05(\mathrm{~m}, 2 \mathrm{H}), 1.94(\mathrm{~s}, 2 \mathrm{H})$. ${ }^{13} \mathrm{C}$ NMR (151 MHz, $\left.\mathrm{CDCl}_{3}\right): \delta 169.2,161.3,130.5,128.1,127.4,113.7,107.2$, $55.5,41.4,22.1,22.0$. IR (neat) $\mathbf{v}_{\text {max }}$ : 2931, 2840, 1714, 1625, 1605, 1508, 1355, 1321, 1248, 1172, 1027, 992, 839, 762, 748, 585. HRMS (ESI $\left.{ }^{+}\right)$: exact mass calculated for $[\mathrm{M}+\mathrm{H}]^{+}\left(\mathrm{C}_{13} \mathrm{H}_{16} \mathrm{NO}_{2}{ }^{+}\right)$requires $218.1181 \mathrm{~m} / \mathrm{z}$, found $218.1174 \mathrm{~m} / \mathrm{z}$.

\section{(2,3-Dihydro-1H-pyrrol-1-yl)(3,4,5-trimethoxyphenyl)methanone (4j)}<smiles>COc1cc(C(=O)N2C=CCC2)cc(OC)c1OC</smiles>

Following GP3 yielded the title compound $(60.6 \mathrm{mg}, 77 \%)$ as colorless oil. No rotameric effects were observed via NMR spectroscopy. ${ }^{1} \mathbf{H} \mathbf{~ N M R ~}(\mathbf{4 0 0} \mathbf{~ M H z}$, $\left.\mathrm{CDCl}_{3}\right): \delta 6.72(\mathrm{~s}, 2 \mathrm{H}), 6.49$ (app s, $\left.1 \mathrm{H}\right), 5.19$ (app s, $\left.1 \mathrm{H}\right), 3.99(\mathrm{t}, J=8.7 \mathrm{~Hz}$, $2 \mathrm{H}), 3.85-3.84(\mathrm{~m}, 9 \mathrm{H}) .2 .76-2.63(\mathrm{~m}, 2 \mathrm{H}) \mathrm{ppm} .{ }^{13} \mathrm{C} \mathrm{NMR}\left(101 \mathrm{MHz}, \mathrm{CDCl}_{3}\right)$ : $\delta 166.7,153.2,139.8,131.1,130.7,112.1,105.1,60.9,56.3,45.8,28.4$ ppm. IR (neat) V max $_{\text {ma }}$ 2862, 2838, 1581, 1506, 1455, 1415, 1320, 1236, 1184, 1125, 1004, 806, 756, 718. HRMS (ESI $\left.{ }^{+}\right)$: exact mass calculated for $[\mathrm{M}+\mathrm{H}]^{+}\left(\mathrm{C}_{14} \mathrm{H}_{18} \mathrm{NO}_{4}{ }^{+}\right)$requires $264.1235 \mathrm{~m} / \mathrm{z}$, found $264.1231 \mathrm{~m} / \mathrm{z}$.

\section{(3,4-Dihydropyridin-1(2H)-yl)(3,4,5-trimethoxyphenyl)methanone (4k)}<smiles>COc1cc(C(=O)N2C=CCCC2)cc(OC)c1OC</smiles>

Following GP3 yielded the title compound $(41.9 \mathrm{mg}, 50 \%)$ as yellow oil. In addition remaining starting material $(30.1 \mathrm{mg}, 37 \%)$ was recovered. The desired enamide was obtained as two rotamers in a ratio of around 4:1. Major isomer: ${ }^{1} \mathrm{H}$ NMR $\left(600 \mathrm{MHz}, \mathrm{CDCl}_{3}\right): \delta 6.68(\mathrm{~s}, 2 \mathrm{H}), 6.53-6.47(\mathrm{~m}, 1 \mathrm{H})$, $4.91-4.80(\mathrm{~m}, 1 \mathrm{H}), 3.86-3.85(\mathrm{~m}, 9 \mathrm{H}), 3.82-3.78(\mathrm{~m}, 2 \mathrm{H}), 2.13-2.09(\mathrm{~m}$, 2H), $1.97-1.91(\mathrm{~m}, 2 \mathrm{H})$ ppm. Minor isomer: ${ }^{1} \mathrm{H}$ NMR (600 MHz, $\left.\mathrm{CDCl}_{3}\right): \delta 7.23$ (app s, $\left.1 \mathrm{H}\right), 6.68(\mathrm{~s}, 2 \mathrm{H})$, $5.28-5.15(\mathrm{~m}, 1 \mathrm{H}), 3.86-3.85(\mathrm{~m}, 9 \mathrm{H}), 3.64-3.53(\mathrm{~m}, 2 \mathrm{H}), 2.11(\mathrm{~m}, 2 \mathrm{H}), 1.85-1.77(\mathrm{~m}, 2 \mathrm{H}) \mathrm{ppm}$. Major isomer: $\left.{ }^{13} \mathrm{C} \mathrm{NMR} \mathrm{(151} \mathrm{MHz,} \mathrm{CDCl}_{3}\right): \delta 169.1,153.2,139.7,130.5,127.6,107.7,105.7,61.0,56.4$, 41.3, 22.0, 21.7 ppm. Minor isomer: ${ }^{13} \mathrm{C}$ NMR could not be clearly identified. IR (neat) $\mathbf{v}_{\text {max }}$ : 1581, 1542, 
1409, 1374, 1356, 1228, 1121, 1002, 761, 735. HRMS (ESI ${ }^{+}$): exact mass calculated for $[\mathrm{M}+\mathrm{H}]^{+}$ $\left(\mathrm{C}_{15} \mathrm{H}_{20} \mathrm{NO}_{4}^{+}\right)$requires $278.1392 \mathrm{~m} / \mathrm{z}$, found $278.1388 \mathrm{~m} / \mathrm{z}$.

\section{Benzo[d][1,3]dioxol-5-yl(3,4-dihydropyridin-1(2H)-yl)methanone (4I)}<smiles>O=C(c1ccc2c(c1)OCO2)N1C=CCCC1</smiles>

Following GP3 yielded the title compound $(41.5 \mathrm{mg}, 60 \%)$ as colorless oil and in addition remaining starting material $(4.0 \mathrm{mg}, 6 \%)$ was recovered. The desired enamide was obtained as two rotamers in a ratio of around 3.6:1. Major isomer: ${ }^{1} \mathrm{H}$ NMR $\left(600 \mathrm{MHz}, \mathrm{CDCl}_{3}\right): \delta 7.02(\mathrm{~d}, J=7.8 \mathrm{~Hz}, 1 \mathrm{H}), 6.98(\mathrm{~s}$, $1 \mathrm{H}), 6.82(\mathrm{~d}, J=8.0 \mathrm{~Hz}, 1 \mathrm{H}), 6.51(\mathrm{app} \mathrm{s}, 1 \mathrm{H}), 6.00(\mathrm{~s}, 2 \mathrm{H}), 4.86$ (app s, 1H), 3.78 (app s, 2H), 2.12 (tdd, $J=6.1,3.8,2.0 \mathrm{~Hz}, 2 \mathrm{H}$ ), 1.94 (app s, 2H) ppm. Minor isomer: ${ }^{1} \mathbf{H} \mathbf{N M R}\left(600 \mathbf{~ M H z}, \mathrm{CDCl}_{3}\right): \delta 7.22$ (app s, $1 \mathrm{H}), 7.02(\mathrm{~d}, J=7.8 \mathrm{~Hz}, 1 \mathrm{H}), 6.98(\mathrm{~s}, 1 \mathrm{H}), 6.82(\mathrm{~d}, J=8.0 \mathrm{~Hz}, 1 \mathrm{H}), 6.00(\mathrm{~s}, 2 \mathrm{H}), 5.21(\mathrm{~s}, 1 \mathrm{H}), 3.63$ (app s, $2 \mathrm{H}), 2.12$ (tdd, $J=6.1,3.8,2.0 \mathrm{~Hz}, 2 \mathrm{H}$ ), 1.85 (app s, 2H) ppm. Major isomer: ${ }^{13} \mathrm{C} \mathrm{NMR}\left(151 \mathrm{MHz}, \mathrm{CDCl}_{3}\right)$ : $\delta$ 168.7, 149.4, 147.6, 128.9, 127.9, 123.3, 109.2, 108.1, 107.5, 101.6, 41.5, 22.1, 21.9 ppm. Minor isomer: ${ }^{13} \mathrm{C}$ NMR could not be clearly identified. IR (neat) $\mathbf{v}_{\text {max }}$ : 2895, 2843, 1633, 1503, 1406, 1375, $1321,1254,1244,1037,996,757$. $\left.\mathrm{HRMS}_{(\mathbf{E S I}}{ }^{+}\right)$: exact mass calculated for $[\mathrm{M}+\mathrm{H}]^{+}\left(\mathrm{C}_{13} \mathrm{H}_{14} \mathrm{NO}_{3}{ }^{+}\right)$requires $232.0974 \mathrm{~m} / \mathrm{z}$, found $232.0968 \mathrm{~m} / \mathrm{z}$.

\section{(2-Bromo-5-methoxyphenyl)(2,3-dihydro-1H-pyrrol-1-yl)methanone (4m)}

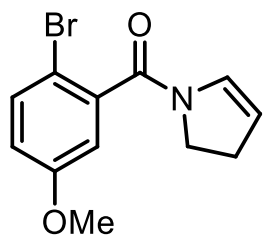

Following GP3 yielded the title compound ( $39.7 \mathrm{mg}$, 47\%) as orange oil. The desired enamide was obtained as two rotamers in a ratio of $4: 1$. Major isomer: ${ }^{1} \mathbf{H}$ NMR (400 MHz, $\left.\mathrm{CDCl}_{3}\right): \delta 7.44(\mathrm{~d}, J=8.7 \mathrm{~Hz}, 1 \mathrm{H}), 6.88-6.78(\mathrm{~m}, 2 \mathrm{H}), 6.01(\mathrm{dt}, J=4.3,2.2$ $\mathrm{Hz}, 1 \mathrm{H}), 5.25-5.20(\mathrm{~m}, 1 \mathrm{H}), 4.06-4.00(\mathrm{~m}, 2 \mathrm{H}), 3.78(\mathrm{~s}, 3 \mathrm{H}), 2.77-2.66(\mathrm{~m}, 2 \mathrm{H})$ ppm. Minor isomer: ${ }^{1} \mathrm{H}$ NMR $\left(\mathbf{4 0 0} \mathrm{MHz}, \mathrm{CDCl}_{3}\right): \delta 7.45(\mathrm{~d}, J=8.6 \mathrm{~Hz}, 1 \mathrm{H}), 7.06(\mathrm{dt}, J$ $=4.3,2.2 \mathrm{~Hz}, 1 \mathrm{H}), 6.88-6.78(\mathrm{~m}, 2 \mathrm{H}), 5.43-5.38(\mathrm{~m}, 1 \mathrm{H}), 3.78(\mathrm{~s}, 3 \mathrm{H}), 3.59(\mathrm{t}, J=8.6 \mathrm{~Hz}, 2 \mathrm{H}), 2.77-$ $2.66(\mathrm{~m}, 2 \mathrm{H}) \mathrm{ppm}$. Major isomer: ${ }^{13} \mathrm{C}$ NMR (151 MHz, CDCl 3 ): $\delta 164.7,159.2,138.7,133.9,129.8,117.4$, 113.6, 113.0, 109.7, 55.8, 45.0, 28.8 ppm. Minor isomer: ${ }^{13} \mathrm{C}$ NMR (151 MHz, $\left.\mathbf{C D C l}_{3}\right): \delta 164.3,159.4$, 139.4, 133.9, 128.8, 117.0, 113.3, 112.9, 109.0, 55.8, 46.8, 29.9 ppm. IR (neat) $\mathbf{v}_{\text {max }}$ : 2961, 2942, 1634, $1618,1450,1424,1236,1177,1161,808,733,599$. HRMS (ESI ${ }^{+}$: exact mass calculated for $[\mathrm{M}+\mathrm{H}]^{+}$ $\left(\mathrm{C}_{12} \mathrm{H}_{13} \mathrm{BrNO}_{2}{ }^{+}\right)$requires $282.0130 \mathrm{~m} / \mathrm{z}$, found $282.0123 \mathrm{~m} / \mathrm{z}$.

\section{(2,3-Dihydro-1H-pyrrol-1-yl)(4-fluorophenyl)methanone (4n)}<smiles>O=C(c1ccc(F)cc1)N1C=CCC1</smiles>

Following GP3 yielded the title compound as colorless oil (37.8 mg, 66\%). The desired enamide was obtained as two rotamers in a ratio of around 6.4:1. Major isomer: ${ }^{1} \mathrm{H}$ NMR $\left(600 \mathrm{MHz}, \mathrm{CDCl}_{3}\right): \delta 7.57-7.50(\mathrm{~m}, 2 \mathrm{H}), 7.11-7.07(\mathrm{~m}, 2 \mathrm{H})$, $6.46-6.38(\mathrm{~m}, 1 \mathrm{H}), 5.25-5.17(\mathrm{~m}, 1 \mathrm{H}), 4.01(\mathrm{t}, J=8.8 \mathrm{~Hz}, 2 \mathrm{H}), 2.73-2.68(\mathrm{~m}$, 2H). Minor isomer: ${ }^{1} \mathrm{H}_{\mathrm{NMR}}\left(600 \mathrm{MHz}, \mathrm{CDCl}_{3}\right): \delta 7.57-7.50(\mathrm{~m}, 2 \mathrm{H}), 7.11-7.07(\mathrm{~m}, 3 \mathrm{H}), 5.37$ (app s, 1H), 3.81 (app s, 2H), $2.73-2.68(\mathrm{~m}, 2 \mathrm{H})$. Major isomer: $\left.{ }^{13} \mathrm{C} \mathrm{NMR} \mathrm{(151} \mathrm{MHz,} \mathrm{CDCl}_{3}\right): \delta 166.0,163.9(\mathrm{~d}$, $J=250.8 \mathrm{~Hz}), 132.0,130.6,130.2(\mathrm{~d}, J=8.5 \mathrm{~Hz}), 115.6(\mathrm{~d}, J=21.8 \mathrm{~Hz}), 112.2,45.9,28.5 \mathrm{ppm} .{ }^{19} \mathrm{~F} \mathrm{NMR}$ (376 MHz, CDCl 3 ): $\delta$-109.3. IR (neat) $\mathbf{v}_{\text {max }}: 1630,1602,1509,1416,1367,1223,1157,1096,1000,845$, $805,756,708,614$. $\left.\mathrm{HRMS}_{(\mathrm{ESI}}{ }^{+}\right)$: exact mass calculated for $[\mathrm{M}+\mathrm{Na}]^{+}\left(\mathrm{C}_{11} \mathrm{H}_{10} \mathrm{FNONa}{ }^{+}\right)$requires 214.0644 $\mathrm{m} / \mathrm{z}$ found $214.0637 \mathrm{~m} / \mathrm{z}$.

\section{(3-Chlorophenyl)(2,3-dihydro-1H-pyrrol-1-yl)methanone (40)}<smiles>O=C(c1cccc(Cl)c1)N1C=CCC1</smiles>

Following GP3 yielded the title compound as colorless oil (29.8 mg, 48\%). The desired enamide was obtained as two rotamers in a ratio of around 6.2:1. Major isomer: ${ }^{1} \mathrm{H}$ NMR $\left(600 \mathrm{MHz}, \mathrm{CDCl}_{3}\right): \delta 7.53-7.49(\mathrm{~m}, 1 \mathrm{H}), 7.44-7.31(\mathrm{~m}, 3 \mathrm{H}), 6.41$ $(\mathrm{dt}, J=4.2,2.1 \mathrm{~Hz}, 1 \mathrm{H}), 5.25-5.20(\mathrm{~m}, 1 \mathrm{H}), 4.05-3.97(\mathrm{~m}, 2 \mathrm{H}), 2.75-2.68(\mathrm{~m}, 2 \mathrm{H})$ ppm. Minor isomer: ${ }^{1} \mathbf{H}$ NMR $\left(600 \mathrm{MHz}, \mathrm{CDCl}_{3}\right): \delta 7.53-7.49(\mathrm{~m}, 1 \mathrm{H}), 7.44-7.31$ (m, 3H), 7.07 (app s, 1H), 5.40 (app s, 1H), $3.80(\mathrm{t}, J=8.2 \mathrm{~Hz}, 2 \mathrm{H}), 2.75-2.68(\mathrm{~m}$, 
2H) ppm. Major isomer: $\left.{ }^{13} \mathrm{C} \mathrm{NMR} \mathrm{(151} \mathrm{MHz,} \mathrm{CDCl}_{3}\right): \delta 165.4,137.6,134.7,130.6,130.3,130.0,128.0$, 125.9, 112.6, 45.8, 28.5. Minor isomer: ${ }^{13} \mathrm{C}$ NMR could not be clearly identified. IR (neat) $\mathbf{v}_{\max }: 1632$, 1611, 1567, 1422, 1402, 1367, 739, 719. HRMS $\left(\mathrm{ESI}^{+}\right)$: exact mass calculated for $[\mathrm{M}+\mathrm{Na}]^{+}$ $\left(\mathrm{C}_{11} \mathrm{H}_{10} \mathrm{ClNONa}{ }^{+}\right)$requires $230.0348 \mathrm{~m} / \mathrm{z}$ found $230.0343 \mathrm{~m} / \mathrm{z}$.

\section{(2,3-Dihydro-1H-pyrrol-1-yl)(4-vinylphenyl)methanone (4p)}<smiles>C=Cc1ccc(C(=O)N2C=CCC2)cc1</smiles>

Following GP3 yielded the title compound (16.4 mg, 27\%) as yellow oil. In addition, remaining starting material $(14.2 \mathrm{mg}, 24 \%)$ was possible to be recovered. The desired enamide was obtained as two rotamers in a ratio of around 5:1. Major isomer: ${ }^{1} \mathbf{H}$ NMR $\left(400 \mathrm{MHz}, \mathrm{CDCl}_{3}\right): \delta 7.49(\mathrm{~d}, J=8.1 \mathrm{~Hz}$, $2 \mathrm{H}), 7.44(\mathrm{~d}, J=8.2 \mathrm{~Hz}, 2 \mathrm{H}), 6.73(\mathrm{dd}, J=17.6,10.9 \mathrm{~Hz}, 1 \mathrm{H}), 6.47(\operatorname{app~s}, 1 \mathrm{H}), 5.81(\mathrm{~d}, J=17.6 \mathrm{~Hz}, 1 \mathrm{H})$, $5.33(\mathrm{~d}, J=10.9 \mathrm{~Hz}, 1 \mathrm{H}), 5.19(\mathrm{app} \mathrm{s}, 1 \mathrm{H}), 4.02(\mathrm{t}, J=8.8 \mathrm{~Hz}, 2 \mathrm{H}), 2.76-2.66(\mathrm{~m}, 2 \mathrm{H}) \mathrm{ppm}$. Minor isomer: ${ }^{1} \mathrm{H}$ NMR (400 MHz, CDCl $): \delta 7.49(\mathrm{~d}, J=8.1 \mathrm{~Hz}, 2 \mathrm{H}), 7.44(\mathrm{~d}, J=8.2 \mathrm{~Hz}, 2 \mathrm{H}), 7.09$ (app s, $1 \mathrm{H}$ ), 6.73 (dd, $J=17.6,10.9 \mathrm{~Hz}, 1 \mathrm{H}), 5.81(\mathrm{~d}, J=17.6 \mathrm{~Hz}, 1 \mathrm{H}), 5.36($ app s, $1 \mathrm{H}), 5.33(\mathrm{~d}, J=10.9 \mathrm{~Hz}, 1 \mathrm{H}), 3.91-3.79(\mathrm{~m}$, 2H), $2.87-2.60(\mathrm{~m}, 2 \mathrm{H})$ ppm. Major isomer: ${ }^{13} \mathrm{C}$ NMR (101 MHz, $\left.\mathrm{CDCl}_{3}\right): \delta 166.8,139.7,136.2,135.1$, $130.8,128.2,126.3,115.7,111.9,45.8,28.5 \mathrm{ppm}$. Minor isomer: ${ }^{13} \mathrm{C}$ NMR could not be clearly identified. IR (neat) $\boldsymbol{v}_{\max }$ : 2953, 2923, 2860, 1629, 1411, 1367, 998, 917, 853, 834, 784, 772, 721. HRMS $\left(\right.$ ESI $\left.^{+}\right)$: exact mass calculated for $[\mathrm{M}+\mathrm{H}]^{+}\left(\mathrm{C}_{13} \mathrm{H}_{14} \mathrm{NO}^{+}\right)$requires $200.1075 \mathrm{~m} / \mathrm{z}$, found $200.1071 \mathrm{~m} / \mathrm{z}$.

\section{(2,3-Dihydro-1H-pyrrol-1-yl)(4-(methylthio)phenyl)methanone (4q)}<smiles>CSc1ccc(C(=O)N2C=CCC2)cc1</smiles>

Following GP3 yielded the title compound (18.0 mg, 27\%) as orange oil. No rotameric effects were observed via NMR spectroscopy. ${ }^{1} \mathbf{H}$ NMR $(400 \mathbf{~ M H z}$, $\mathrm{CDCl}_{3}$ ): $\delta 7.44(\mathrm{~d}, J=7.9 \mathrm{~Hz}, 2 \mathrm{H}), 7.25(\mathrm{~d}, J=8.1 \mathrm{~Hz}, 2 \mathrm{H}), 6.47$ (app s, $\left.1 \mathrm{H}\right)$, 5.19 (app s, $1 \mathrm{H}$ ), 4.00 (t, $J=8.7 \mathrm{~Hz}, 2 \mathrm{H}), 2.73-2.67(\mathrm{~m}, 2 \mathrm{H}), 2.50(\mathrm{~s}, 3 \mathrm{H}) .{ }^{13} \mathrm{C}$ NMR (101 MHz, CDCl $): \delta 166.6,142.1,132.2,130.8,128.4,125.7,111.9$, 45.9, 28.5, 15.3 ppm. IR (neat) $\mathbf{v}_{\text {max }}$ : 2953, 2858, 1410, 1367, 1186, 1090, 831, 751, 712. HRMS (ESI ${ }^{+}$): exact mass calculated for $[\mathrm{M}+\mathrm{H}]^{+}\left(\mathrm{C}_{12} \mathrm{H}_{14} \mathrm{NOS}^{+}\right)$requires $220.0796 \mathrm{~m} / \mathrm{z}$, found $220.0789 \mathrm{~m} / \mathrm{z}$.

\section{3-(2,3-Dihydro-1H-pyrrole-1-carbonyl)benzonitrile (4r)}<smiles>N#Cc1cccc(C(=O)N2C=CCC2)c1</smiles>

Following GP3 yielded the title compound $(34.0 \mathrm{mg}, 57 \%)$ as yellow solid. In addition, remaining starting material $(26.0 \mathrm{mg}, 43 \%)$ was possible to be recovered. The desired enamide was obtained as two rotamers in a ratio of around 6:1. Major isomer: ${ }^{1} \mathbf{H}$ NMR $\left(600 \mathrm{MHz}, \mathrm{CDCl}_{3}\right): \delta 7.79(\mathrm{~s}, 1 \mathrm{H}), 7.75-7.70(\mathrm{~m}, 2 \mathrm{H}), 7.55(\mathrm{t}, \mathrm{J}=$ $7.8 \mathrm{~Hz}, 1 \mathrm{H}), 6.34(\mathrm{dt}, J=4.1,2.0 \mathrm{~Hz}, 1), 5.31-5.27(\mathrm{~m}, 1 \mathrm{H}), 4.04-4.00(\mathrm{~m}, 2 \mathrm{H}), 2.75$ $-2.71(\mathrm{~m}, 2 \mathrm{H})$. Minor isomer: ${ }^{1} \mathbf{H}$ NMR $\left(600 \mathrm{MHz}, \mathrm{CDCl}_{3}\right): \delta 7.79(\mathrm{~s}, 1 \mathrm{H}), 7.75-7.70(\mathrm{~m}, 2 \mathrm{H}), 7.55(\mathrm{t}, J=$ $7.8 \mathrm{~Hz}, 1 \mathrm{H}), 7.05(\mathrm{app} \mathrm{s}, 1 \mathrm{H}), 5.44(\mathrm{app} \mathrm{s}, 1 \mathrm{H}), 3.79(\mathrm{t}, \mathrm{J}=8.3 \mathrm{~Hz}, 2 \mathrm{H}), 2.75-2.71(\mathrm{~m}, 2 \mathrm{H})$. Major isomer: ${ }^{13} \mathrm{C} \mathrm{NMR}\left(151 \mathrm{MHz}, \mathrm{CDCl}_{3}\right): \delta 164.4,137.2,133.8,132.1,131.4,129.7,129.6,118.0,113.5,113.0,46.0$, 28.5. Minor isomer: ${ }^{13} \mathrm{C}$ NMR could not be clearly identified. IR (neat) $\mathbf{v}_{\text {max }}: 2923,2897,2861,1632$, $1612,1433,1403,1367,1198,1181,804,737,690$. HRMS (ESI $^{+}$): exact mass calculated for $[\mathrm{M}+\mathrm{H}]^{+}$ $\left(\mathrm{C}_{12} \mathrm{H}_{11} \mathrm{~N}_{2} \mathrm{O}^{+}\right)$requires $199.0871 \mathrm{~m} / \mathrm{z}$, found $199.0866 \mathrm{~m} / \mathrm{z}$.

\section{(2,3-Dihydro-1H-pyrrol-1-yl)(ferrocene)methanone (4u)}

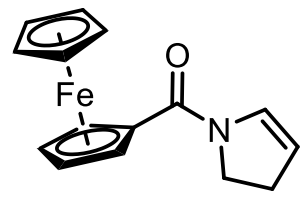

Following GP3 using as a cosolvent DCM $(0.9 \mathrm{~mL})$ yielded the title compound (45.3 mg, 54\%) as a red solid. ${ }^{1} \mathrm{H} \mathrm{NMR}\left(400 \mathrm{MHz}, \mathrm{CDCl}_{3}\right): \delta 7.12(\mathrm{dt}, J=4.2,2.1 \mathrm{~Hz}$, 1H), $5.24(\mathrm{app} \mathrm{s}, 1 \mathrm{H}), 4.79-4.60(\mathrm{~m}, 2 \mathrm{H}), 4.35(\mathrm{~s}, 2 \mathrm{H}), 4.23(\mathrm{~s}, 5 \mathrm{H}), 4.09-3.93$ (m, 2H), $2.81-2.59(\mathrm{~m}, 2 \mathrm{H}) \mathrm{ppm} .{ }^{13} \mathrm{C}$ NMR (176 MHz, $\left.\mathrm{CDCl}_{3}\right): \delta 167.0,130.8$, 111.0, 77.8, 70.3, 69.9, 69.8, 46.4, 28.0 ppm. IR (neat) $\mathbf{v}_{\text {max }}$ : 1604, 1459, 1406, 
1346, 809. HRMS (ESI $\left.{ }^{+}\right)$: exact mass calculated for $[\mathrm{M}+\mathrm{H}]^{+}\left(\mathrm{C}_{15} \mathrm{H}_{16} \mathrm{FeNO}^{+}\right)$requires $\mathrm{m} / \mathrm{z} 282.0576$, found $\mathrm{m} / \mathrm{z} 282.0576$.

4-(2,3-Dihydro-1H-pyrrole-1-carbonyl)-2-methoxyphenyl methylthiazole-5-carboxylate (4v)

2-(3-cyano-4-isobutoxyphenyl)-4-<smiles>COc1cc(C(=O)N2C=CCC2)ccc1OC(=O)c1sc(-c2ccc(OCC(C)C)c(C#N)c2)nc1C</smiles>

Following GP3 (0.24 mmol scale) using as a cosolvent DCM $(1.1 \mathrm{~mL})$ yielded the title compound $(47.6 \mathrm{mg}, 38 \%)$ as a yellow solid. In addition, remaining starting material $(17.2 \mathrm{mg}$, 14\%) was recovered. ${ }^{1} \mathrm{H}$ NMR $(\mathbf{4 0 0} \mathbf{~ M H z}$, $\left.\mathrm{CDCl}_{3}\right): \delta 8.23(\mathrm{~d}, J=2.2 \mathrm{~Hz}, 1 \mathrm{H}), 8.13(\mathrm{dd}, J=$ $8.8,2.2 \mathrm{~Hz}, 1 \mathrm{H}), 7.24-7.18(\mathrm{~m}, 2 \mathrm{H}), 7.12(\mathrm{~d}, J=$ $8.0 \mathrm{~Hz}, 1 \mathrm{H}), 7.03(\mathrm{~d}, J=8.9 \mathrm{~Hz}, 1 \mathrm{H}), 6.54(\mathrm{~s}, 1 \mathrm{H})$, $5.23(\mathrm{~s}, 1 \mathrm{H}), 4.03(\mathrm{t}, J=8.8 \mathrm{~Hz}, 2 \mathrm{H}), 3.91(\mathrm{~d}, J=6.5 \mathrm{~Hz}, 2 \mathrm{H}), 3.87(\mathrm{~s}, 3 \mathrm{H}), 2.82(\mathrm{~s}, 3 \mathrm{H}), 2.77-2.70(\mathrm{~m}$, $2 \mathrm{H}), 2.26-2.17(\mathrm{~m}, 1 \mathrm{H}), 1.10(\mathrm{~d}, J=6.7 \mathrm{~Hz}, 6 \mathrm{H}) \mathrm{ppm} .{ }^{13} \mathrm{C} \mathrm{NMR}\left(101 \mathrm{MHz}, \mathrm{CDCl}_{3}\right): \delta 168.4,166.1,163.4$, $162.8,159.8,151.6,140.9,135.0,132.8,132.4,130.7,126.0,122.9,120.3,120.2,115.5,112.8,112.5$, $112.3,103.2,75.9,56.3,46.0,28.5,28.3,19.2,17.8$ ppm. IR (neat) v $\max _{2}$ 2960, 2929, 2873, 2229, 1733, 1605, 1507, 1423, 1371, 1329, 1273, 1250, 1199, 1182, 1121, 1049, 1011, 733. HRMS (ESI'): exact mass calculated for $[\mathrm{M}+\mathrm{H}]^{+}\left(\mathrm{C}_{28} \mathrm{H}_{28} \mathrm{~N}_{3} \mathrm{O}_{5} \mathrm{~S}^{+}\right)$requires $\mathrm{m} / \mathrm{z} 518.1744$, found $\mathrm{m} / \mathrm{z} 518.1751$. 


\subsection{Unsuccessful and low-yielding Substrates}

Table 3. Unsuccessful and poor yielding substrates. ${ }^{a}$

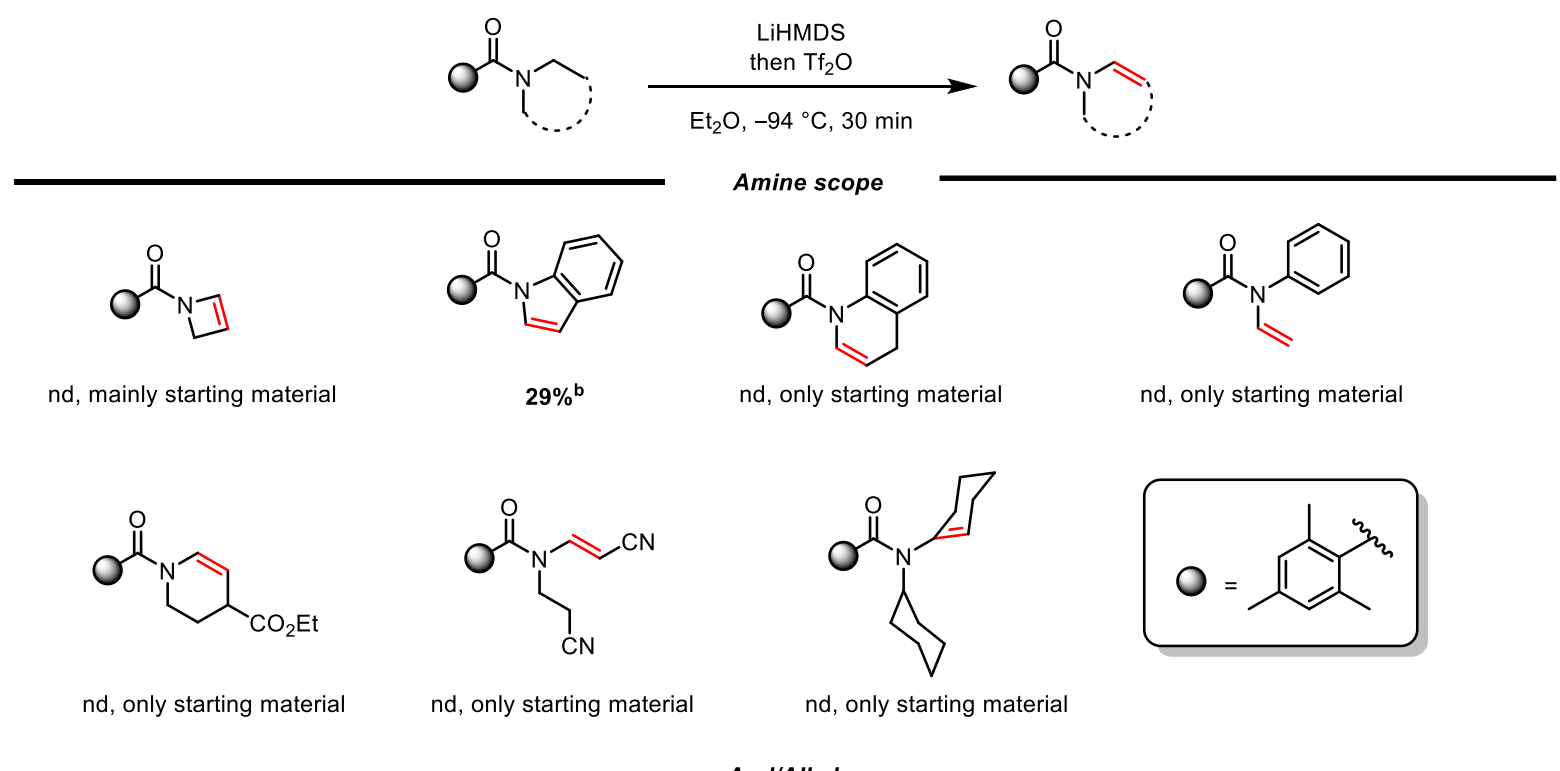<smiles>O=C(c1c(Cl)cc(Cl)cc1Cl)N1C=CCC1</smiles>

traces, rest starting material

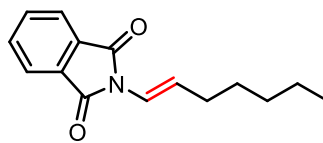

nd, only starting material<smiles>CC(=O)N1C=CCC1</smiles>

nd, only starting material<smiles>O=C(c1ccc([N+](=O)[O-])cc1)N1C=CCCC1</smiles>

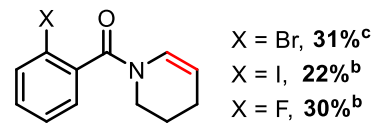

traces, rest starting material<smiles>CC(C)(C)OC(=O)N1C=CCC1</smiles>

nd, only starting material<smiles>O=C(N1CCCC1)C(c1ccccc1)(c1ccccc1)c1ccccc1</smiles>

nd, only starting material

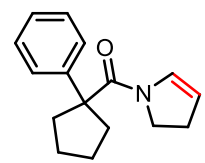

nd, only starting materia

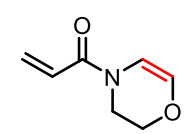

nd, only starting material<smiles>O=C(/C=C/c1ccccc1)N1C=CCC1</smiles>

$12 \%^{c}$

a amide $(0.30 \mathrm{mmol}), 1.5 \mathrm{~mL} \mathrm{Et}_{2} \mathrm{O}$, LiHMDS $\left(0.96 \mathrm{mmol}, 1 \mathrm{M}\right.$ solution in THF) with slow addition, $\mathrm{Tf}_{2} \mathrm{O}(0.48 \mathrm{mmol})$ with addition over $1 \mathrm{~min}$. ${ }^{b}$

$\mathrm{GC}$ yield determined with decane (ratio 1:1) as internal standard. ${ }^{\mathrm{c}}$ Isolated yield. 


\subsection{Application of Enamides}

Methyl 1-benzoyl-8-oxo-2,3,3a,4,7,7a-hexahydro-1H-4,7-(epoxymethano)indole-5-carboxylate (5a)<smiles>O=C(c1ccccc1)N1C=CCC1</smiles>
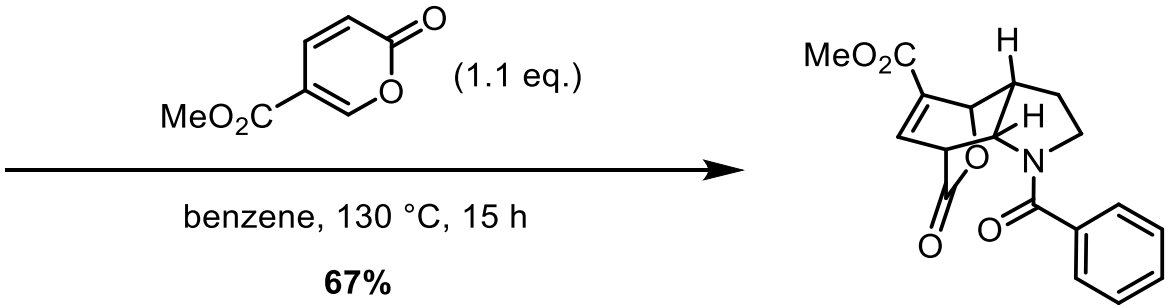

A procedure adopted from literature was used. ${ }^{[18]}$ An oven-dried screw-cap vial was loaded with methyl coumalate $(84.8 \mathrm{mg}, 0.55 \mathrm{mmol}, 1.10 \mathrm{eq}$.), followed by the addition of enamide $4 \mathrm{c}(86.6 \mathrm{mg}$, $0.50 \mathrm{mmol}, 1.00$ eq.) dissolved in toluene $(1 \mathrm{~mL})$. The mixture was heated at reflux $\left(130^{\circ} \mathrm{C}\right.$, oil bath temperature) for $15 \mathrm{~h}$, after which full conversion of the enamide was observed by TLC. The solvent was evaporated and the crude material was subjected to flash column chromatography (heptane/EtOAc, 8:2 - 2:8) to give the title compound (110 mg, $0.34 \mathrm{mmol}, 67 \%)$ as white solid. ${ }^{1} \mathbf{H}$ NMR $\left(400 \mathrm{MHz}, \mathrm{CDCl}_{3}\right): \delta 7.44-7.31(\mathrm{~m}, 5 \mathrm{H}), 7.25(\mathrm{~d}, J=5.0 \mathrm{~Hz}, 1 \mathrm{H}), 5.56(\mathrm{~s}, 1 \mathrm{H}), 4.74(\mathrm{~d}, J=6.2 \mathrm{~Hz}$, $1 \mathrm{H}), 4.26(\mathrm{~s}, 1 \mathrm{H}), 3.75(\mathrm{~s}, 3 \mathrm{H}), 3.66-3.53(\mathrm{~m}, 1 \mathrm{H}), 3.46-3.35(\mathrm{~m}, 1 \mathrm{H}), 2.68(\mathrm{t}, J=9.3 \mathrm{~Hz}, 1 \mathrm{H}), 2.20-$ 2.00 (m, 2H) ppm. $\left.{ }^{13} \mathrm{C} \mathrm{NMR} \mathrm{(101} \mathrm{MHz,} \mathrm{CDCl}_{3}\right): \delta 167.6,154.2,134.7,131.7,131.5,128.6,127.8,127.0$, 123.1, 112.8, 112.6, 112.2, 100.5, 55.9, 40.5, 25.4 ppm. IR (neat) $\mathbf{v}_{\text {max }}$ 3106, 2941, 2872, 2342, 1644, 1524, 1446, 1345, 1168, 1088, 1077, 983, 856, 740, 680, 606, 558. HRMS (ESI ${ }^{+}$): exact mass calculated for $[\mathrm{M}+\mathrm{H}]^{+}\left(\mathrm{C}_{18} \mathrm{H}_{18} \mathrm{NO}_{5}{ }^{+}\right)$requires $328.1185 \mathrm{~m} / \mathrm{z}$, found $328.1176 \mathrm{~m} / \mathrm{z}$.

\section{Ethyl 1-benzoyl-4-(4-chlorophenyl)-2,3-dihydro-1H-pyrrolo[3,2-c]quinoline-8-carboxylate (5b)}<smiles>O=C(c1ccccc1)N1CCCC1</smiles>

1)<smiles>CCOC(=O)c1ccc(N)cc1</smiles>

$(1.0$ eq. $)+$

$$
\begin{aligned}
& \mathrm{Sc}(\mathrm{OTf})_{3}(0.2 \text { eq. }) \\
& \mathrm{MeCN}, \mathrm{rt}, 3 \mathrm{~d}
\end{aligned}
$$

2) $\mathrm{DDQ}(2.0$ eq.)

$\mathrm{CHCl}_{3}, \mathrm{rt}, 16 \mathrm{~h}$

$40 \%$

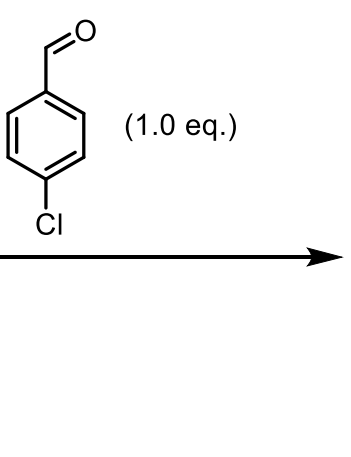<smiles>CCOC(=O)c1ccc2nc(-c3ccc(Cl)cc3)c3c(c2c1)N(C(=O)c1ccccc1)CC3</smiles>

A modified procedure adopted from literature was used. ${ }^{[19]}$ Ethyl 4-aminobenzoate $(49.6 \mathrm{mg}$, $0.30 \mathrm{mmol}, 1.00$ eq.) and $p$-chlorobenzaldehyde $(42.2 \mathrm{mg}, 0.30 \mathrm{mmol}, 1.00$ eq.) were dissolved in anhydrous acetonitrile $(0.5 \mathrm{~mL})$ in an oven-dried round bottom flask. Then, molecular sieves ( $4 \AA$ ) were added, followed by scandium triflate $(29.5 \mathrm{mg}, 0.06 \mathrm{mmol}, 0.20$ eq.). The resulting mixture was stirred at room temperature for $5 \mathrm{~min}$. Then, the enamide $(52.0 \mathrm{mg}, 0.30 \mathrm{mmol}, 1.00 \mathrm{eq}$.) dissolved in acetonitrile $(0.5 \mathrm{~mL})$ was added. The mixture was stirred for $3 \mathrm{~d}$ at room temperature. Then, the reaction was stopped by the addition of aqueous saturated solution of $\mathrm{NaHCO}_{3}(5 \mathrm{~mL})$ and filtered. The aqueous phase was extracted with EtOAc $(3 \times 10 \mathrm{~mL})$. The combined organic phases were dried over anhydrous $\mathrm{MgSO}_{4}$ and the solvent was removed under reduced pressure. The crude material was used directly in the next oxidative step and was dissolved in anhydrous $\mathrm{CHCl}_{3}(0.5 \mathrm{~mL})$. Next, 3-dichloro-5,6dicyano-1,4-benzoquinone ( $136 \mathrm{mg}, 0.60 \mathrm{mmol}, 2.00 \mathrm{eq}$.) was added. The reaction was stirred for $16 \mathrm{~h}$ at room temperature. Then $\mathrm{CH}_{2} \mathrm{Cl}_{2}(5 \mathrm{~mL})$ was added and the organic phase was washed with saturated aqueous $\mathrm{NaHCO}_{3}(3 \times 5 \mathrm{~mL})$, filtered over anhydrous $\mathrm{MgSO}_{4}$ and concentrated under reduced pressure. The obtained material was purified by column chromatography (heptane/EtOAc, 1:9-1:1) yielding the title compound ( $55.5 \mathrm{mg}, 0.121 \mathrm{mmol}, 41 \%)$ as yellow solid. ${ }^{1} \mathrm{H}$ NMR $\left(400 \mathrm{MHz}, \mathrm{CDCl}_{3}\right): \delta 8.54(\mathrm{~s}, 1 \mathrm{H})$, $8.22(\mathrm{~d}, J=8.9 \mathrm{~Hz}, 1 \mathrm{H}), 8.14(\mathrm{~d}, J=8.9 \mathrm{~Hz}, 1 \mathrm{H}), 7.81(\mathrm{app} \mathrm{t}, 4 \mathrm{H}), 7.57(\mathrm{t}, J=7.6 \mathrm{~Hz}, 1 \mathrm{H}), 7.48(\mathrm{~m}, 4 \mathrm{H})$, 
$\left.4.36(\mathrm{~m}, 4 \mathrm{H}), 3.35(\mathrm{t}, J=7.6 \mathrm{~Hz}, 2 \mathrm{H}), 1.37(\mathrm{t}, J=7.0 \mathrm{~Hz}, 3 \mathrm{H}) \mathrm{ppm} .{ }^{13} \mathrm{C} \mathrm{NMR} \mathrm{(101} \mathrm{MHz,} \mathrm{CDCl}\right): \delta 171.1$, $166.3,156.1,150.8,150.3,137.7,135.6,134.8,132.3,130.1,130.0,129.1,129.0,128.8,128.8,128.6$, $127.4,124.9,118.5,61.3,54.9,30.3,14.5$ ppm. IR (neat) $\mathbf{v}_{\text {max }}$ : 3062, 2980, 2343, 1713, 1662, 1489, 1149, 1391, 1253, 1092, 1014, 843, 730, 706. HRMS (ESI $\left.{ }^{+}\right)$: exact mass calculated for $[\mathrm{M}+\mathrm{H}]^{+}$ $\left(\mathrm{C}_{27} \mathrm{H}_{22} \mathrm{ClN}_{2} \mathrm{O}_{3}{ }^{+}\right)$requires $457.1313 \mathrm{~m} / \mathrm{z}$, found $\mathrm{m} / \mathrm{z} 457.1305$.

\section{Mesityl(3,4,4a,8b-tetrahydrobenzo[3,4]cyclobuta[1,2-b]pyridin-1(2H)-yl)methanone (5c)}<smiles>Cc1cc(C)c(C(=O)N2C=CCCC2)c(C)c1</smiles>

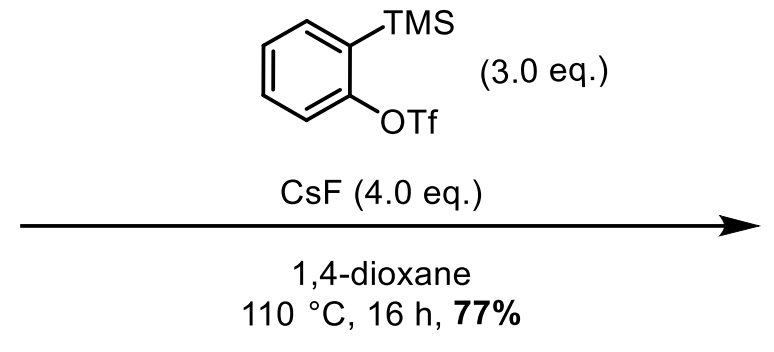<smiles>Cc1cc(C)c(C(=O)N2CCCC3c4ccccc4C32)c(C)c1</smiles>

A modified procedure adopted from literature was used. ${ }^{[20]}$ A screw-cap vial was loaded with ovendried CsF (91.1 mg, $0.60 \mathrm{mmol}, 4.00$ eq.), enamide $2 \mathrm{a}$ ( $34.3 \mathrm{mg}, 0.15 \mathrm{mmol}, 1.00$ eq.) and anhydrous 1,4-dioxane $(2 \mathrm{~mL})$. Then, 2-(trimethylsilyl)phenyl trifluoro-methanesulfonate $(115 \mu \mathrm{L}, 0.45 \mathrm{mmol}$, 3.00 eq.) was added dropwise using a gastight syringe. The reaction mixture was heated at $110{ }^{\circ} \mathrm{C}$ for $16 \mathrm{~h}$. After cooling to room temperature, the solution was filtered through a plug of Celite ${ }^{\circledR}$, followed by three rinse cycles with EtOAc. The filtrate was concentrated in vacuo and subsequently purified via silica gel flash column chromatography (heptane/EtOAc, pure heptane to 6:4) giving rise to the desired amidobenzocyclobutane $(35.3 \mathrm{mg}, 0.116 \mathrm{mmol}, 77 \%)$ as yellowish oil. The desired cyclobutane product was obtained as two isomers in a ratio of $1: 1 .{ }^{1} \mathbf{H}$ NMR $\left(600 \mathrm{MHz}, \mathrm{CDCl}_{3}\right): \delta 7.35-7.21(\mathrm{~m}, 2.5 \mathrm{H}), 7.12$ (dd, $J=7.1,0.5 \mathrm{~Hz}, 0.5 \mathrm{H}), 7.09-7.06(\mathrm{~m}, 1 \mathrm{H}), 6.88-6.83(\mathrm{~m}, 2 \mathrm{H}), 5.98(\mathrm{~d}, J=5.5 \mathrm{~Hz}, 0.5 \mathrm{H}), 4.92(\mathrm{~d}, J=$ $5.5 \mathrm{~Hz}, 0.5 \mathrm{H}), 4.28-4.19(\mathrm{~m}, 0.5 \mathrm{H}), 4.15-4.11(\mathrm{~m}, 0.5 \mathrm{H}), 3.95-3.92(\mathrm{~m}, 0.5 \mathrm{H}), 3.30$ (ddd, $J=13.8$, 8.0, $1.7 \mathrm{~Hz}, 0.5 \mathrm{H}), 3.15-3.11(\mathrm{~m}, 0.5 \mathrm{H}), 3.08-3.01(\mathrm{~m}, 0.5 \mathrm{H}), 2.39(\mathrm{~s}, 1.5 \mathrm{H}), 2.29-2.25(\mathrm{~m}, 6 \mathrm{H}), 2.17$ $(\mathrm{s}, 1.5 \mathrm{H}), 2.10-2.03(\mathrm{~m}, 1 \mathrm{H}), 2.00-1.92(\mathrm{~m}, 1 \mathrm{H}), 1.70-1.61(\mathrm{~m}, 0.5 \mathrm{H}), 1.51-1.45(\mathrm{~m}, 0.5 \mathrm{H}), 1.43-$ $1.37(\mathrm{~m}, 0.5 \mathrm{H}), 1.35-1.30(\mathrm{~m}, 0.5 \mathrm{H}) \mathrm{ppm} .{ }^{13} \mathrm{C}$ NMR (151 MHz, $\left.\mathrm{CDCl}_{3}\right): \delta 172.1,171.6,146.9,146.3$, $146.1,145.1,138.2,138.0,134.3,134.2,133.7,133.5,133.3,133.2,129.5,129.0,128.5,128.4,128.3$, $127.9,127.9,123.8,123.0,122.9,122.1,56.5,52.6,45.2,44.0,43.1,39.4,25.7,24.7,19.9,19.4,18.9$, 17.2 ppm. IR (neat) $\mathbf{v}_{\text {max }}: 2919,2876,2855,1629,1611,1436,1404,1361,1308,1263,1169,852,756$. HRMS (ESI ${ }^{+}$): exact mass calculated for $[\mathrm{M}+\mathrm{H}]^{+}\left(\mathrm{C}_{21} \mathrm{H}_{24} \mathrm{NO}^{+}\right)$requires $306.1858 \mathrm{~m} / \mathrm{z}$, found 306.1855 $\mathrm{m} / \mathrm{z}$.

\section{$\mathrm{N}$-(2-(5-Methoxy-1H-indol-3-yl)ethyl)benzamide (5d)}<smiles>O=C(c1ccccc1)N1C=CCC1</smiles>

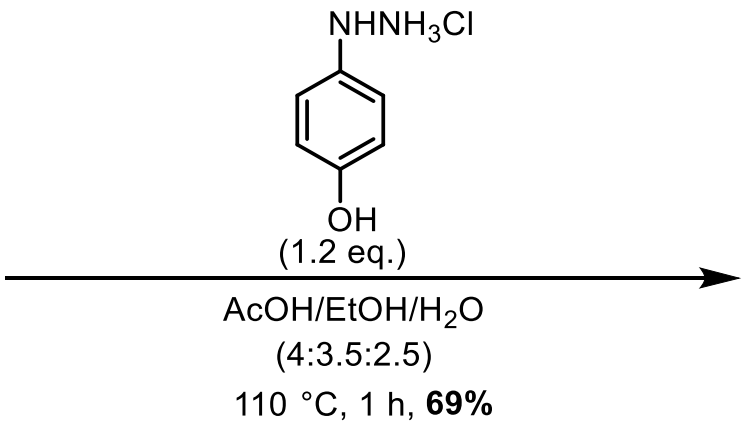<smiles>COc1ccc2[nH]cc(CCNC(=O)c3ccccc3)c2c1</smiles>

A modified procedure adopted from literature was used. ${ }^{[21]}$ An oven-dried screw-cap vial was loaded with enamide $4 \mathrm{c}(41.6 \mathrm{mg}, 0.24 \mathrm{mmol}, 1.00$ eq.), (4-methoxyphenyl)hydrazine hydrochloride $(50.6 \mathrm{mg}$, $0.29 \mathrm{mmol}, 1.20$ eq.) and a solvent mixture of $\mathrm{AcOH} / \mathrm{EtOH} / \mathrm{H}_{2} \mathrm{O}(0.5 \mathrm{~mL}, 0.7 \mathrm{~mL}, 0.8 \mathrm{~mL})$. The reaction mixture was heated at $110^{\circ} \mathrm{C}$ for $1 \mathrm{~h}$. After cooling to room temperature, $\mathrm{CH}_{2} \mathrm{Cl}_{2}(5 \mathrm{~mL})$ was added and the organic phase was washed with saturated aqueous $\mathrm{NaHCO}_{3}(5 \mathrm{~mL})$ dried over anhydrous $\mathrm{MgSO}_{4}$ 
and evaporated in vacuo. The crude material was subjected to flash column chromatography (heptane/EtOAc, 8:2 - 2:8) affording the title compound $(49.1 \mathrm{mg}, 0.167 \mathrm{mmol}, 70 \%)$ as a red liquid. ${ }^{1} \mathrm{H}$ NMR $\left(400 \mathrm{MHz}, \mathrm{CDCl}_{3}\right): \delta 8.21(\mathrm{~s}, 1 \mathrm{H}), 7.59(\mathrm{~d}, J=7.5 \mathrm{~Hz}, 2 \mathrm{H}), 7.37(\mathrm{t}, J=7.3 \mathrm{~Hz}, 1 \mathrm{H}), 7.28(\mathrm{t}, J=7.5$ $\mathrm{Hz}, 2 \mathrm{H}), 7.16(\mathrm{~d}, J=8.7 \mathrm{~Hz}, 1 \mathrm{H}), 6.94(\mathrm{~d}, J=11.7 \mathrm{~Hz}, 2 \mathrm{H}), 6.77(\mathrm{dd}, J=8.7,1.7 \mathrm{~Hz}, 1 \mathrm{H}), 6.26(\mathrm{~s}, 1 \mathrm{H}), 3.71$ - $3.67(\mathrm{~m}, 5 \mathrm{H}), 2.96(\mathrm{t}, J=6.6 \mathrm{~Hz}, 2 \mathrm{H}) \mathrm{ppm} .{ }^{13} \mathrm{C}$ NMR (101 MHz, $\left.\mathrm{CDCl}_{3}\right): \delta 167.6,154.2,134.7,131.7$, $131.5,128.6,127.8127 .0,123.1,112.8,112.6,112.2,100.5,55.9,40.5,25.4 \mathrm{ppm}$. IR (neat) $v_{\max }: 3406$, 3296, 3058, 2933, 1635, 1577, 1529, 1484, 1453, 1439, 1293, 1214, 1173, 1072, 1030, 922, 797, 710. HRMS (ESI $\left.{ }^{+}\right)$: exact mass calculated for $[\mathrm{M}+\mathrm{H}]^{+}\left(\mathrm{C}_{18} \mathrm{H}_{19} \mathrm{~N}_{2} \mathrm{O}_{2}{ }^{+}\right)$requires $295.1447 \mathrm{~m} / \mathrm{z}$, found 295.1441 $\mathrm{m} / \mathrm{z}$.

\section{$\mathrm{N}$-(3,3-difluoropropyl)benzamide (5e)}<smiles>O=C(c1ccccc1)N1C=CCC1</smiles>

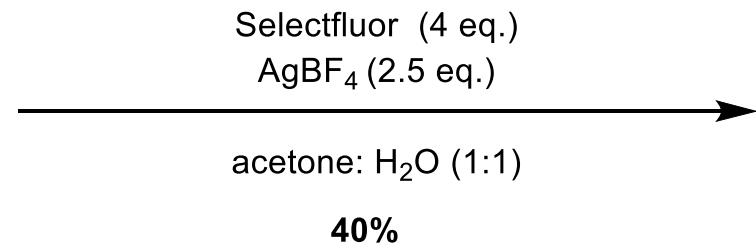<smiles>O=C(NCCC(F)F)c1ccccc1</smiles>

A modified procedure adopted from literature was used. ${ }^{[22]}$ An oven-dried dram vial was loaded with enamide $4 c\left(26.3 \mathrm{mg}, 0.15 \mathrm{mmol}, 1.00\right.$ eq.) dissolved in acetone $/ \mathrm{H}_{2} \mathrm{O}(1: 1,0.5 \mathrm{~mL})$. Selectfluor ( $213 \mathrm{mg}, 0.60 \mathrm{mmol}, 4.00$ eq.) was added followed by $\mathrm{AgBF}_{4}(73 \mathrm{mg}, 0.38 \mathrm{mmol}, 2.5 \mathrm{eq})$. The reaction mixture was stirred at room temperature for $16 \mathrm{~h}$. After this time, water $(5 \mathrm{~mL})$ was added and the aqueous phase was extracted with EtOAc $(5 \times 10 \mathrm{~mL})$. The combined organic layers were dried over anhydrous $\mathrm{MgSO}_{4}$ and concentrated under reduced pressure. The crude material was purified via flash column chromatography (heptane/EtOAc, pure heptane to 6:4) to afford the title compound (12.0 mg, $0.06 \mathrm{mmol}, 40 \%)$ as a colorless oil. ${ }^{1} \mathrm{H}$ NMR $\left(600 \mathrm{MHz}, \mathrm{CDCl}_{3}\right): \delta 7.77-7.74(\mathrm{~m}, 2 \mathrm{H}), 7.53-7.49(\mathrm{~m}$, $1 \mathrm{H}), 7.45-7.41(\mathrm{~m}, 2 \mathrm{H}), 6.41(\mathrm{~s}, 1 \mathrm{H}), 5.99(\mathrm{tt}, J=56.1,4.1 \mathrm{~Hz}, 1 \mathrm{H}), 3.70-3.65(\mathrm{~m}, 2 \mathrm{H}), 2.26-2.14(\mathrm{~m}$, 2H) ppm. ${ }^{13} \mathrm{C}$ NMR $\left(151 \mathrm{MHz}, \mathrm{CDCl}_{3}\right): \delta 167.8,134.4,131.8,128.8,127.0,116.6(\mathrm{t}, J=239.3 \mathrm{~Hz}), 34.1$ $(\mathrm{t}, J=20.6 \mathrm{~Hz}), 33.8(\mathrm{t}, J=6.2 \mathrm{~Hz}) \mathrm{ppm} .{ }^{19} \mathbf{F}$ NMR $\left(376 \mathrm{MHz}, \mathrm{CDCl}_{3}\right): \delta-116.30 \mathrm{ppm}$. IR (neat) $\mathbf{v}_{\text {max }}: 3321$, 3066, 2942, 1710, 1639, 1537, 1308, 1294, 1221, 1117, 1067, 966, 693, 670, 595. HRMS (ESI ${ }^{+}$): exact mass calculated for $[\mathrm{M}+\mathrm{Na}]^{+}\left(\mathrm{C}_{10} \mathrm{H}_{12} \mathrm{NONa}^{+}\right)$requires $222.0706 \mathrm{~m} / \mathrm{z}$, found $222.0695 \mathrm{~m} / \mathrm{z}$.

(R)-8-Hydroxy-7,9-dimethoxy-1,2,3,9b-tetrahydro-5H-pyrrolo[2,1-a]isoindol-5-one (5f)<smiles>COc1cc(C(=O)N2C=CCC2)cc(OC)c1OC</smiles>

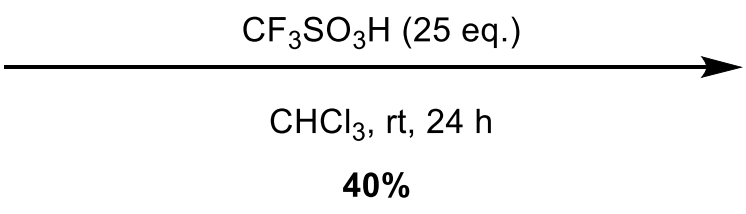

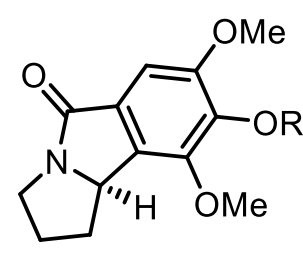

$\mathrm{R}=\mathrm{H}$ and $\mathrm{OMe}$ ratio 9:1

A modified procedure adopted from literature was used. ${ }^{[18]}$ To a solution of the enamide $(105 \mathrm{mg}, 0.40$ mmol, 1.00 eq.) dissolved in $\mathrm{CHCl}_{3}(2 \mathrm{~mL}$ ) trifluoromethanesulfonic acid ( $1.95 \mathrm{~mL}, 22.0 \mathrm{mmol}, 25.0$ eq.) was added slowly. The reaction was stirred for $16 \mathrm{~h}$ before it was poured into ice water $(10 \mathrm{~mL})$. The aqueous phase was extracted three times with $\mathrm{CHCl}_{3}(5 \mathrm{~mL})$, the organic phases were combined and dried over anhydrous $\mathrm{MgSO}_{4}$. After concentration of the organic phase under reduced pressure, the crude material was subjected to flash column chromatography (heptane/EtOAc, 1:1 to pure EtOAc) affording the cyclized product ( $39.4 \mathrm{mg}, 0.158 \mathrm{mmol}, 40 \%$ ) as yellow solid. The major product was that of demethylation of the para-methoxy group. ${ }^{1} \mathrm{H}$ NMR $\left(600 \mathrm{MHz}, \mathrm{CDCl}_{3}\right): \delta 7.06(\mathrm{~s}, 1 \mathrm{H}), 6.07(\mathrm{~s}, 1 \mathrm{H})$, 
$4.66(\mathrm{dd}, J=10.6,5.5 \mathrm{~Hz}, 1 \mathrm{H}), 3.98(\mathrm{~s}, 3 \mathrm{H}), 3.92(\mathrm{~s}, 3 \mathrm{H}), 3.69-3.59(\mathrm{~m}, 1 \mathrm{H}), 3.41-3.35(\mathrm{~m}, 1 \mathrm{H}), 2.39-$ $2.25(\mathrm{~m}, 3 \mathrm{H}), 1.25-1.16(\mathrm{~m}, 1 \mathrm{H}) \mathrm{ppm} .{ }^{13} \mathrm{C}$ NMR (151 MHz, $\left.\mathrm{CDCl}_{3}\right): \delta 172.2,148.9,141.9,141.6,132.3$, $124.9,101.2,62.9,60.6,56.8,42.0,30.1,29.4$ ppm. IR (neat) $\mathbf{v}_{\text {max }}$ : 3221, 2940, 2893, 2848, 1666, 1617, $1477,1373,1327,1224,1192,1095,1071,1031$. HRMS (ESI ${ }^{+}$: exact mass calculated for $[\mathrm{M}+\mathrm{H}]^{+}$ $\left(\mathrm{C}_{13} \mathrm{H}_{16} \mathrm{NO}_{4}{ }^{+}\right)$requires $250.1079 \mathrm{~m} / \mathrm{z}$, found $250.1065 \mathrm{~m} / \mathrm{z}$.

\section{Mesityl(5-phenyl-3,4-dihydropyridin-1(2H)-yl)methanone (5g)}<smiles>Cc1cc(C)c(C(=O)N2C=CCCC2)c(C)c1</smiles>

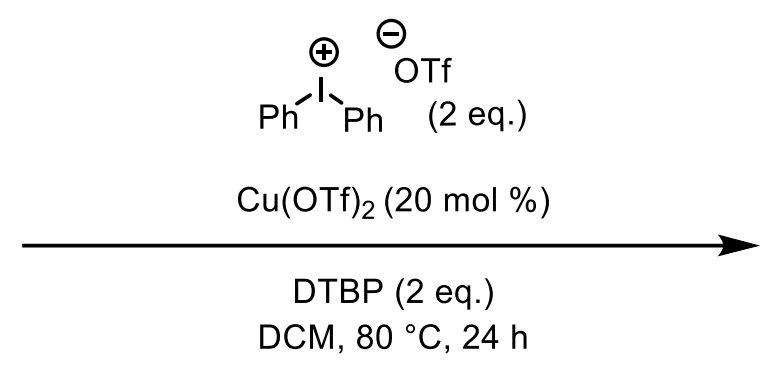

$24 \%$<smiles>Cc1cc(C)c(C(=O)N2C=C(c3ccccc3)CCC2)c(C)c1</smiles>

A modified procedure adopted from literature was used. ${ }^{[23]}$ An oven-dried screw cap vial was loaded with diphenyliodonium trifuoromethanesulfonate $(258 \mathrm{mg}, 0.60 \mathrm{mmol}, 2.00 \mathrm{eq}$.) and copper(II) trifluoromethanesulfonate $(21.7 \mathrm{mg}, 0.06 \mathrm{mmol}, 0.20$ eq.). Then, enamide $2 \mathrm{a}(68.8 \mathrm{mg}, 0.30 \mathrm{mmol}$, 1.00 eq.) dissolved in $\mathrm{CH}_{2} \mathrm{Cl}_{2}(0.5 \mathrm{~mL})$ was added followed by the addition of 2,6-di-tert-butylpyridine (139 $\mathrm{\mu L}, 0.60 \mathrm{mmol}, 2.00$ eq.). The resulting mixture was heated at $80^{\circ} \mathrm{C}$ for $24 \mathrm{~h}$. Then, a saturated aqueous solution of $\mathrm{NaHCO}_{3}(5 \mathrm{~mL})$ was added. The aqueous phase was extracted with $\mathrm{CH}_{2} \mathrm{Cl}_{2}(2 \times 5 \mathrm{~mL})$ and the combined organic phases were washed with brine $(5 \mathrm{~mL})$. The organic phase was dried over anhydrous $\mathrm{MgSO}_{4}$ and concentrated in vacuo. The crude product was purified by column chromatography (heptane/EtOAc, pure heptane to 8:2) giving rise to the title compound as colorless oil (21.6 mg, $0.07 \mathrm{mmol}, 24 \%)$. The NMR analysis revealed the presence of two rotamers in a ratio of 1.5:1. ${ }^{1} \mathrm{H}$ NMR $\left(400 \mathrm{MHz}, \mathrm{CDCl}_{3}\right): \delta 7.98(\mathrm{~s}, 0.4 \mathrm{H}), 7.54-7.45(\mathrm{~m}, 1 \mathrm{H}), 7.34(\mathrm{t}, J=7.6 \mathrm{~Hz}, 1 \mathrm{H}), 7.23(\mathrm{~d}$, $1 \mathrm{H}), 7.19-7.09(\mathrm{~m}, 2 \mathrm{H}), 6.87(\mathrm{~s}, 2 \mathrm{H}), 6.57(\mathrm{~s}, 0.6 \mathrm{H}), 4.00-3.94(\mathrm{~m}, 1.2 \mathrm{H}), 3.34-3.29(\mathrm{~m}, 0.8 \mathrm{H}), 2.54$ $(\mathrm{t}, J=5.8 \mathrm{~Hz}, 2 \mathrm{H}), 2.30(\mathrm{~s}, 3 \mathrm{H}), 2.23-2.19(\mathrm{~m}, 6 \mathrm{H}), 2.09-2.03(\mathrm{~m}, 1.2 \mathrm{H}), 1.97-1.92(\mathrm{~m}, 0.8 \mathrm{H}) .{ }^{13} \mathrm{C}$ NMR (151 MHz, CDCl (1): $\delta_{170.1}$ 169.1, 139.9, 139.7, 138.7, 138.5, 134.1, 133.8, 133.5, 132.7, 128.6 (2C), $128.4,126.9,126.7,124.84$ (2C), 123.2, 121.3, 121.1, 119.7, 44.2 40.0, 25.1, 24.8, 22.7 (2C), 21.3 (2C), 19.2, 19.1. IR (neat) $\mathbf{v}_{\text {max }}: 2922,2855,2362,1628,1611,1455,1386,1312,1261,1170,996,851,750$, 727, 694. HRMS $\left(\mathrm{ESI}^{+}\right)$: exact mass calculated for $[\mathrm{M}+\mathrm{H}]^{+}\left(\mathrm{C}_{21} \mathrm{H}_{24} \mathrm{NO}^{+}\right)$requires $306.1858 \mathrm{~m} / \mathrm{z}$, found $306.1854 \mathrm{~m} / \mathrm{z}$. 


\section{Mechanistic Studies}

\subsection{Isotopic-labeling Experiments:}

\subsubsection{Preparation of Isotopically-labeled Starting Materials}

\section{Phenyl(pyrrolidin-1-yl)methanone- ${ }^{18} \mathrm{O}(6 \mathrm{a})$}<smiles>O=C(c1ccccc1)N1CCCC1</smiles>

$3 c$

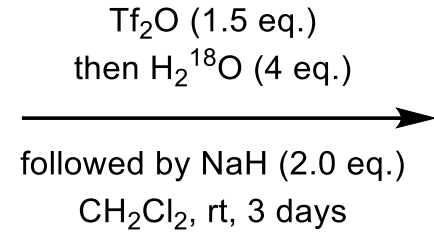

$44 \%$<smiles>O=C(c1ccccc1)N1CCCC1</smiles>

$6 a$

Amide (3c, $200 \mathrm{mg}, 1.14 \mathrm{mmol}, 1.00 \mathrm{eq}$ ) was dissolved in anhydrous $\mathrm{CH}_{2} \mathrm{Cl}_{2}$ (3.5 mL) in a flame-dried flask. The resulting solution was cooled to $0{ }^{\circ} \mathrm{C}$ and $\mathrm{Tf}_{2} \mathrm{O}(288 \mu \mathrm{L}, 1.71 \mathrm{mmol}, 1.50 \mathrm{eq})$ was added. The reaction was stirred for $15 \mathrm{~min}$ at $0{ }^{\circ} \mathrm{C}$. Then, ${ }^{18} \mathrm{O}$-water $(82 \mu \mathrm{L}, 4.57 \mathrm{mmol}, 4.00 \mathrm{eq})$ was added and the mixture was stirred for $24 \mathrm{~h}$ at room temperature. Since no conversion of the iminium triflate was observed, sodium hydride (60\% in paraffine, $91.3 \mathrm{mg}, 2.28 \mathrm{mmol}, 2.00 \mathrm{eq}$ ) was added and the mixture was stirred vigorously for $3 \mathrm{~d}$. The mixture was diluted with $\mathrm{CH}_{2} \mathrm{Cl}_{2}(30 \mathrm{~mL})$, washed with $\mathrm{NH}_{4} \mathrm{Cl}$ (saturated, $20 \mathrm{~mL}$ ), dried over anhydrous $\mathrm{MgSO}_{4}$ and concentrated under reduced pressure. The crude product was purified by column chromatography (heptane/EtOAc, 8:2 to pure EtOAc) to afford 6a (89.9 $\mathrm{mg}, 44 \%$ ) with an ${ }^{18} \mathrm{O}$ content of $75 \%$, as determined by HRMS.

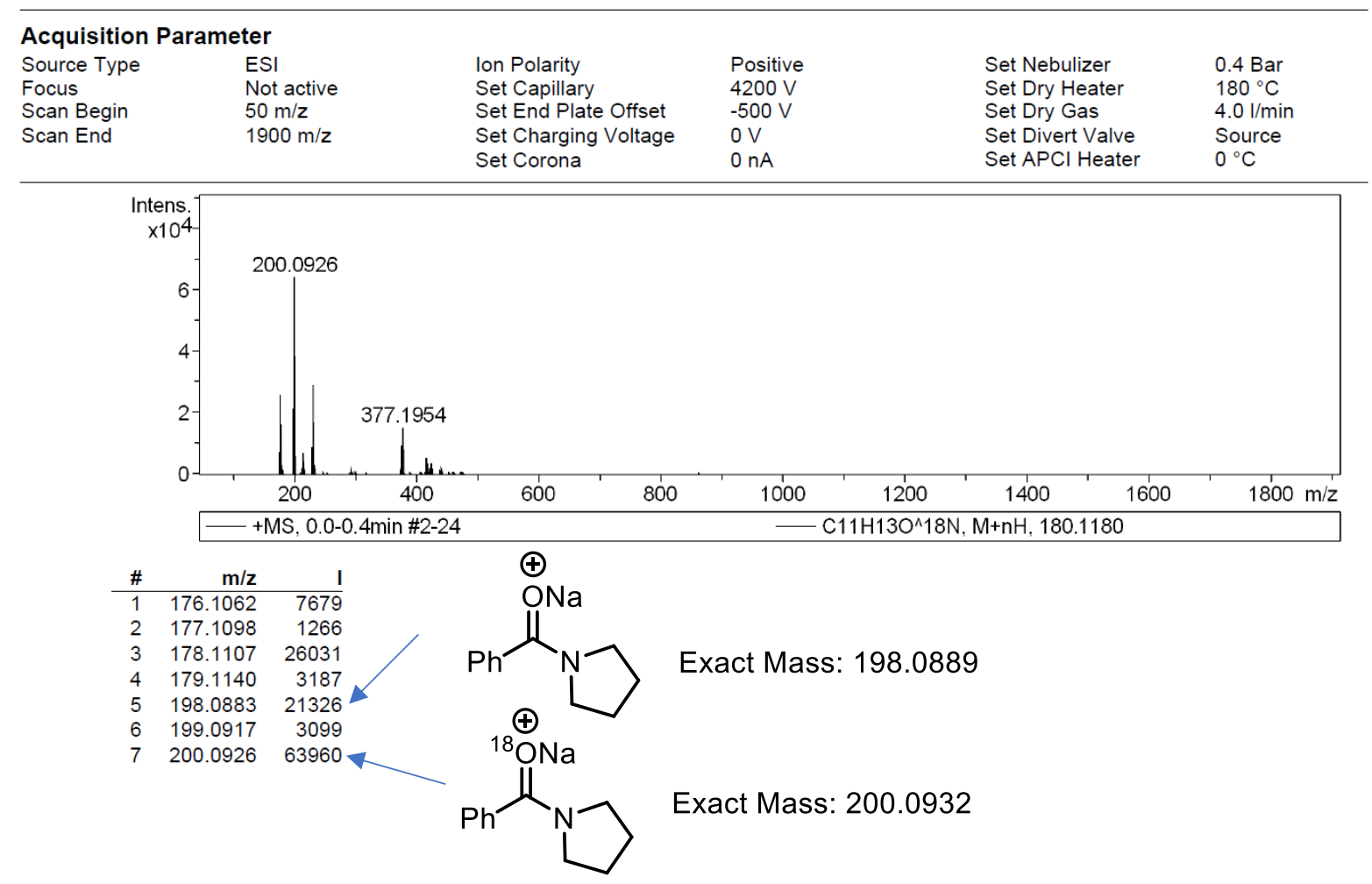

The (non-labeled) product is reported in the literature. ${ }^{[3]}$ 


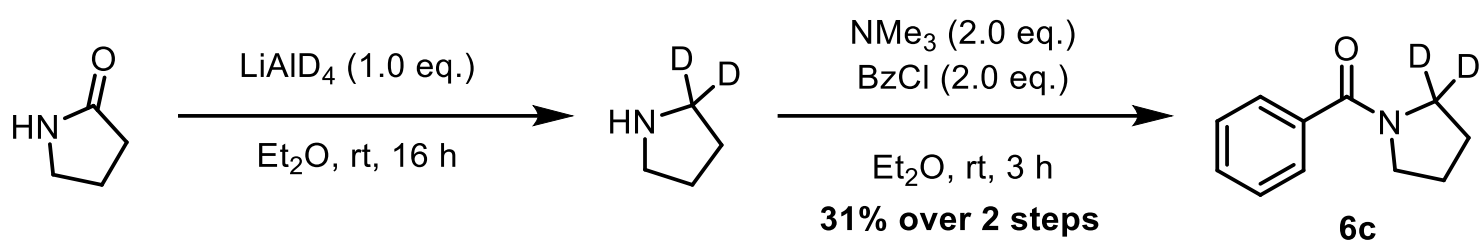

A modified procedure adopted from literature was used. ${ }^{[24]}$ Lithium aluminium deuteride $(703 \mathrm{mg}$, $16.7 \mathrm{mmol}, 1.00$ eq.) was suspended in anhydrous $\mathrm{Et}_{2} \mathrm{O}(50 \mathrm{~mL})$ in a flame-dried flask $(250 \mathrm{~mL})$. The resulting suspension was cooled to $0{ }^{\circ} \mathrm{C}$ and 2 -pyrrolidone $(1.27 \mathrm{~mL}, 1.43 \mathrm{~g}, 16.7 \mathrm{mmol}, 1.00$ eq.) was added dropwise. The reaction mixture was stirred for $16 \mathrm{~h}$ at room temperature. The reaction was stopped by addition of a saturated solution of sodium sulfate until evolution of hydrogen ceased. The resulting clear solution was filtered from the off-white precipitate and the filtrate was collected in a separate flame-dried flask $(250 \mathrm{~mL})$. Then, benzoyl chloride $(3.89 \mathrm{~mL}, 33.5 \mathrm{mmol}, 2.00$ eq.) and trimethylamine $(4.67 \mathrm{~mL}, 33.5 \mathrm{mmol}, 2.00$ eq.) were added to the filtrate, and the mixture was stirred for $3 \mathrm{~h}$ at room temperature. The mixture was transferred to a separation funnel by using $\mathrm{Et}_{2} \mathrm{O}(50 \mathrm{~mL})$. The organic layer was extracted with $1 \mathrm{~N} \mathrm{HCl}(100 \mathrm{~mL})$ and saturated $\mathrm{Na}_{2} \mathrm{CO}_{3}(100 \mathrm{~mL})$, dried over anhydrous $\mathrm{MgSO}_{4}$ and concentrated. The crude product was purified by column chromatography (heptane/EtOAc, 8:2 to pure EtOAc) to afford $904 \mathrm{mg}$ (31\%) of the deuterated product. The spectra were found to be in accordance with the literature. ${ }^{[24]}$
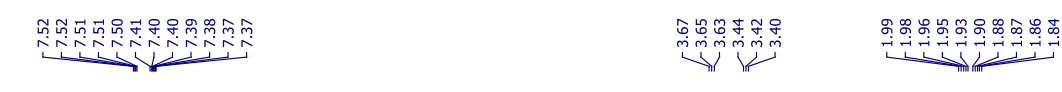<smiles>[2H]C1([2H])CCCN1C(=O)c1ccccc1</smiles>

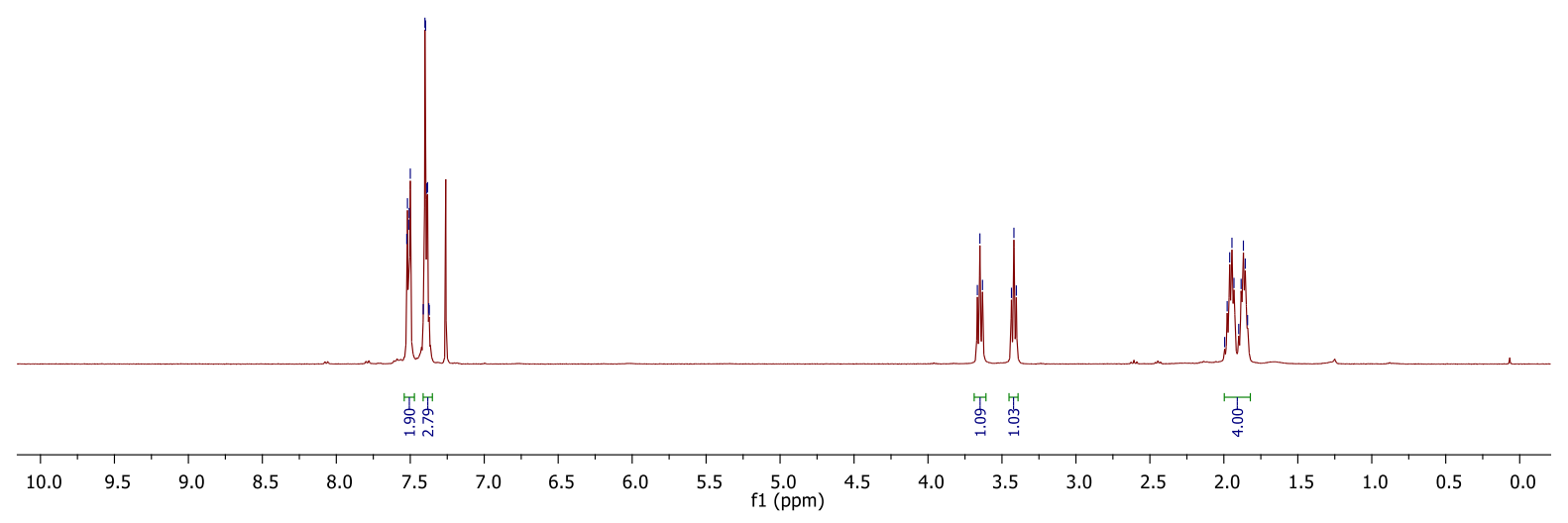




\section{$N$-(4 $\lambda^{12}$-buta-1,3-diyn-1-yl-d9)-N-butyl-2,4,6-trimethylbenzamide (6f)}

$\begin{array}{ll}\text { Following the GP1A (2.0 mmol) using benzoyl chloride and dibutylamine- } \\ \mathrm{N}_{\mathrm{N}^{-}}-\mathrm{C}_{4} \mathrm{H}_{9} & \begin{array}{l}\text { (monobutyl-d }) \text { (purchased from Sigma Aldrich) afforded the desired amide } \\ \mathrm{C}_{4} \mathrm{D}_{9}\end{array} \\ (535 \mathrm{mg}, 1.88 \mathrm{mmol}, 94 \%) \text { as colorless oil. }\end{array}$

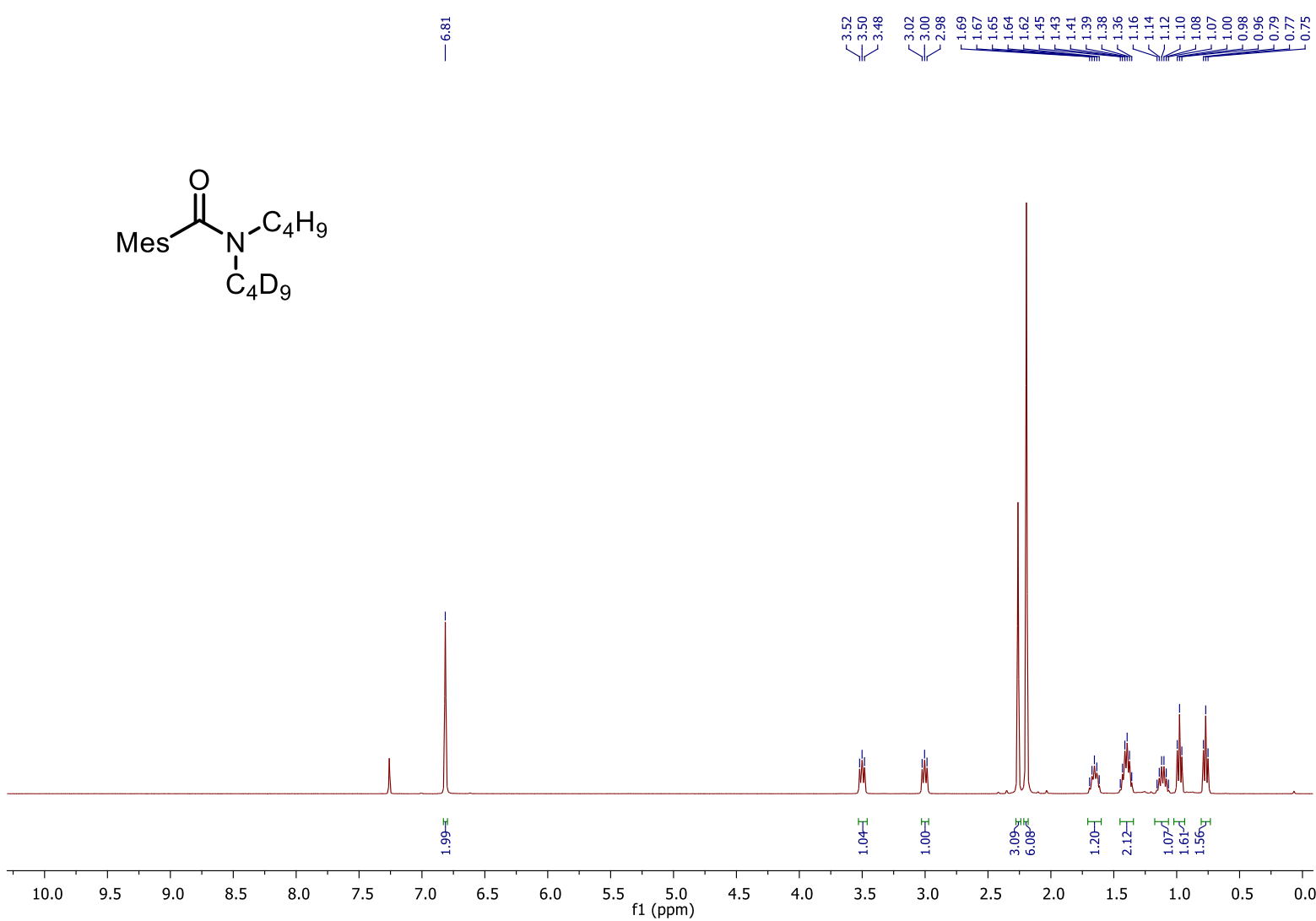




\subsubsection{N-Dehydrogenation Reaction with Isotopically-labeled Substrates}

Scheme 1. Overview of isotope-labeling experiments.

a)<smiles>O=C([18O])N1CCCC1</smiles>

$6 \mathbf{a}$ $\left(75 \%{ }^{18} \mathrm{O}\right)$

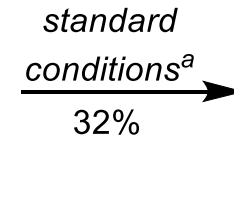<smiles>O=C([18O])N1C=CCC1</smiles>

$6 \mathbf{b}$

$$
\left(77 \%{ }^{18} \mathrm{O}\right)
$$

b)

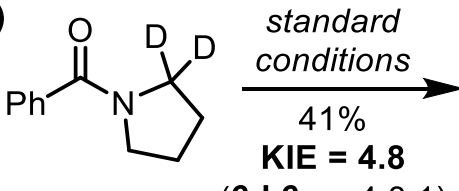

$6 c$

$(6 \mathrm{~d}: 6 \mathrm{e}=4.8: 1)$<smiles>[2H]C1([2H])CC=CN1C(=O)c1ccccc1</smiles>

6d<smiles>[2H]C1=CCCN1C(=O)c1ccccc1</smiles>

$6 e$

c)

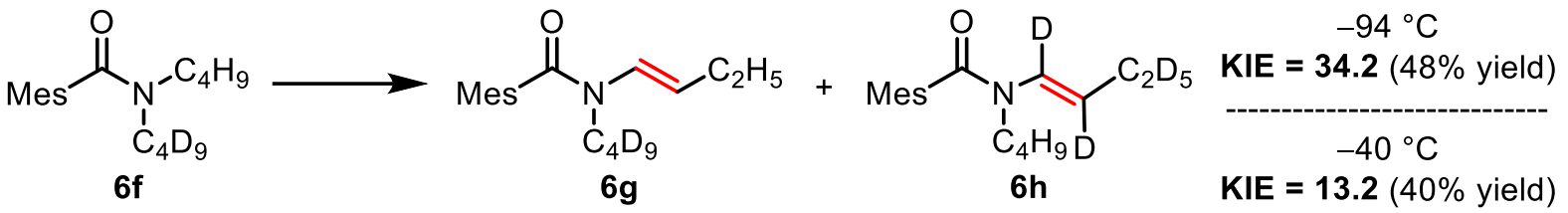

Substrates $6 \boldsymbol{a}, \mathbf{6 c}$ and $\boldsymbol{6 f}$ have been conducted to GP3; ${ }^{a}$ deviation from standard conditions: $-78^{\circ} \mathrm{C}$ and THF.

\section{Experiment A}<smiles>O=C(c1ccccc1)N1C=CCC1</smiles>

Following GP3 (deviation: $-78{ }^{\circ} \mathrm{C}$ and THF as solvent) using $6 a$ yielded the desired enamide as a colorless oil (17.9 mg, $32 \%)$. HRMS analysis revealed an ${ }^{18} \mathrm{O}$ content of

$6 \mathrm{~b}$ $77 \%$.

$\left(77 \%{ }^{18} \mathrm{O}\right)$

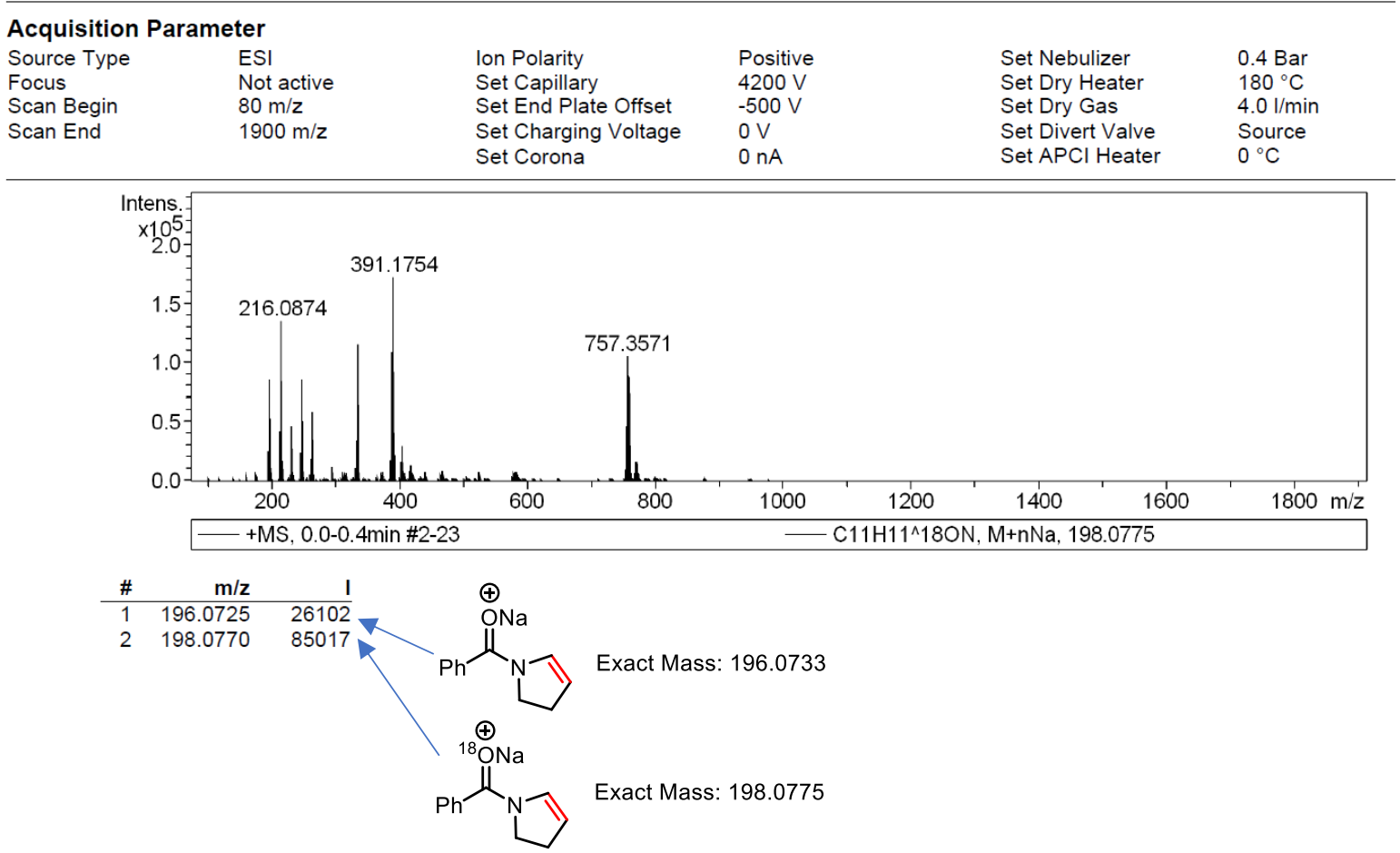




\section{Experiment B}

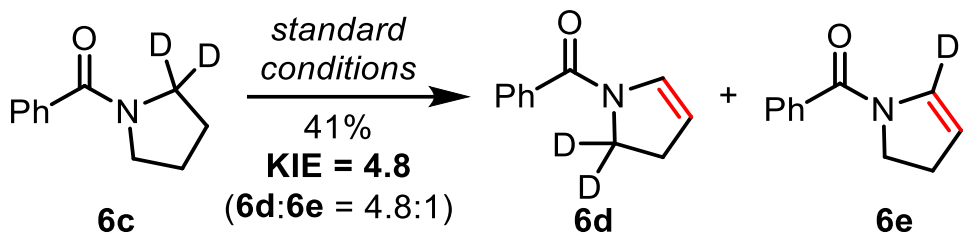

Following GP3 using $6 c$ yielded the desired enamide as a colorless oil $(23.3 \mathrm{mg}, 41 \%)$. For the calculation of the KIE value, the ratio of $\mathbf{6} \mathbf{d}$ to $6 \mathbf{e}$ was determined by NMR spectroscopy as well as HMRS analysis.<smiles>[2H]C1([2H])C[C+]=CN1C(=O)c1ccccc1</smiles>

$6 d$<smiles>O=C(c1ccccc1)N1[C][C][C][C]1Cl</smiles>

$6 e$

KIE values based on different protons of $6 \mathrm{~d} / 6 \mathrm{e}$ :

for $\mathrm{a}=4.8$

for $\mathrm{d}^{\prime}=3.9$

with two rotamers with two rotamers
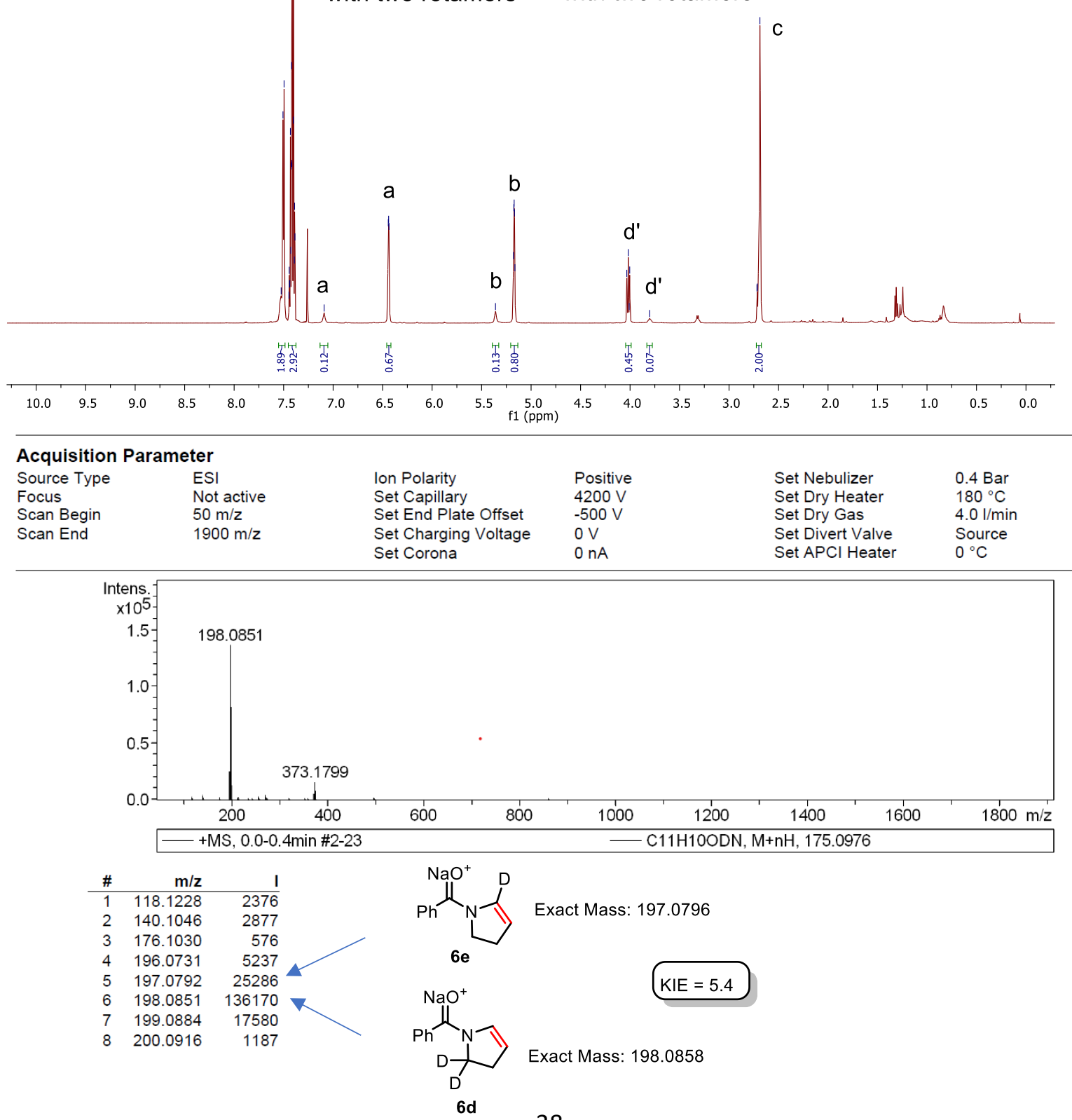


\section{Experiment C}

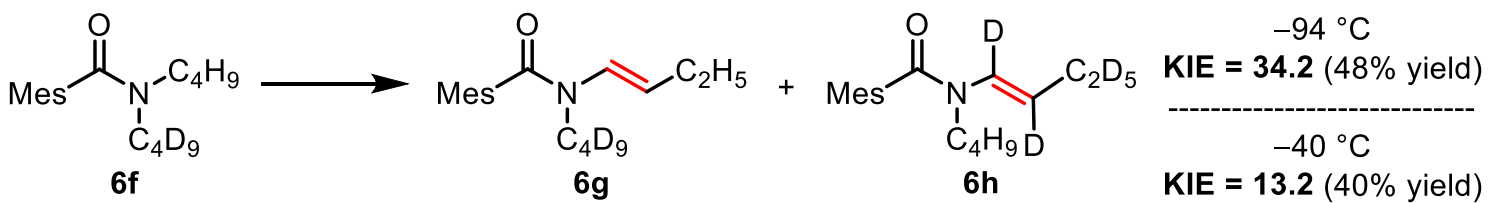

Following GP3 $\left(-94^{\circ} \mathrm{C}\right)$ using $6 f$ yielded the desired enamide as a colorless oil (40.7 mg, $48 \%$ ). For the calculation of the KIE value, the ratio $6 \mathrm{~g} / 6 \mathrm{ff}$ was determined by NMR spectroscopy as well as HMRS analysis.

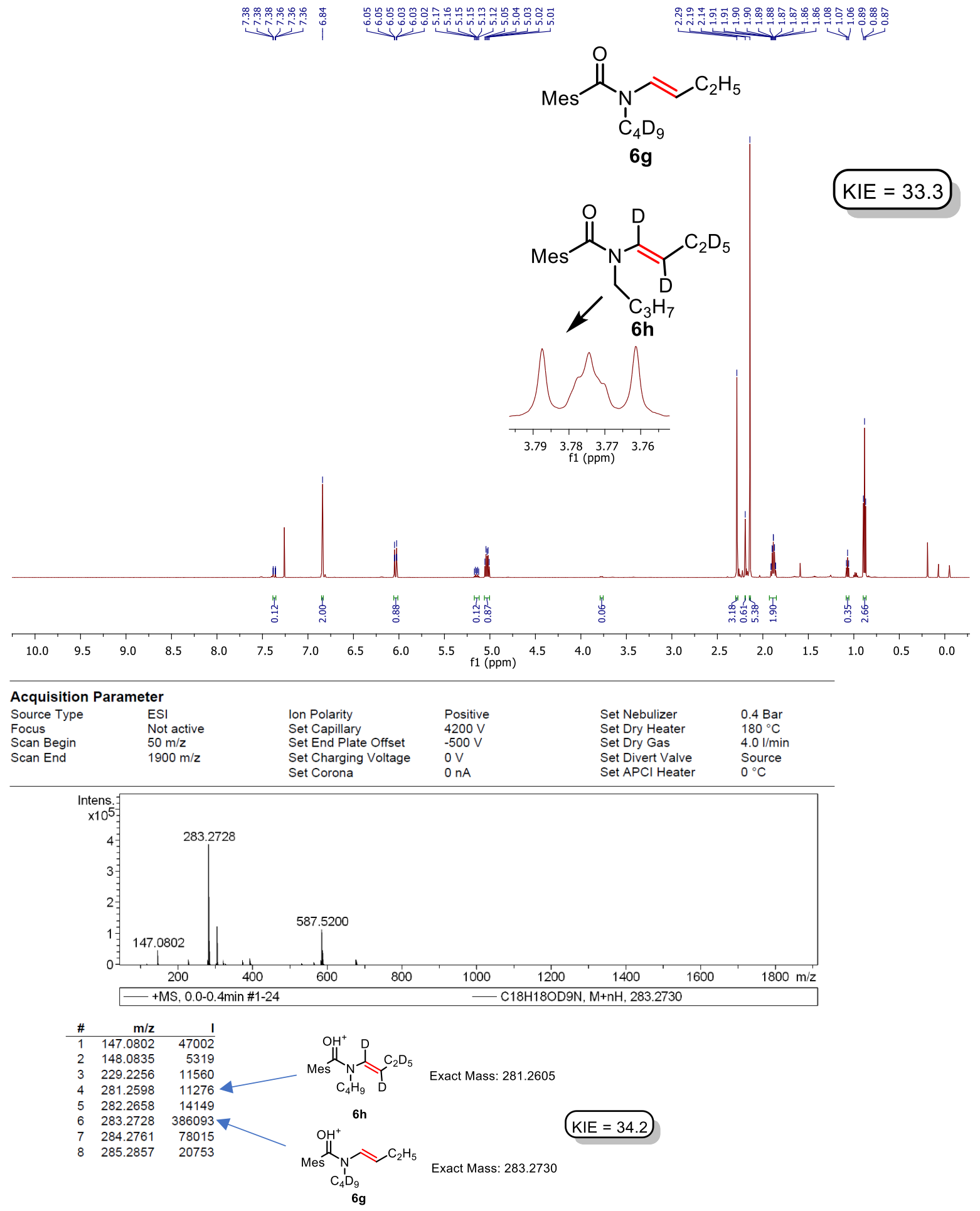


In order to check the temperature effect on the KIE for substrate $6 f$, the same reaction ( $0.2 \mathrm{mmol}$ scale) was performed at $-41{ }^{\circ} \mathrm{C}$ (cooling system: MeCN + dry ice) to provide $6 \mathrm{~g} / 6 \mathrm{ff}(33.6 \mathrm{mg}, 40 \%)$. For the calculation of the KIE value, the ratio $\mathbf{6 g} / \mathbf{6 f}$ was determined by NMR spectroscopy as well as HMRS analysis.

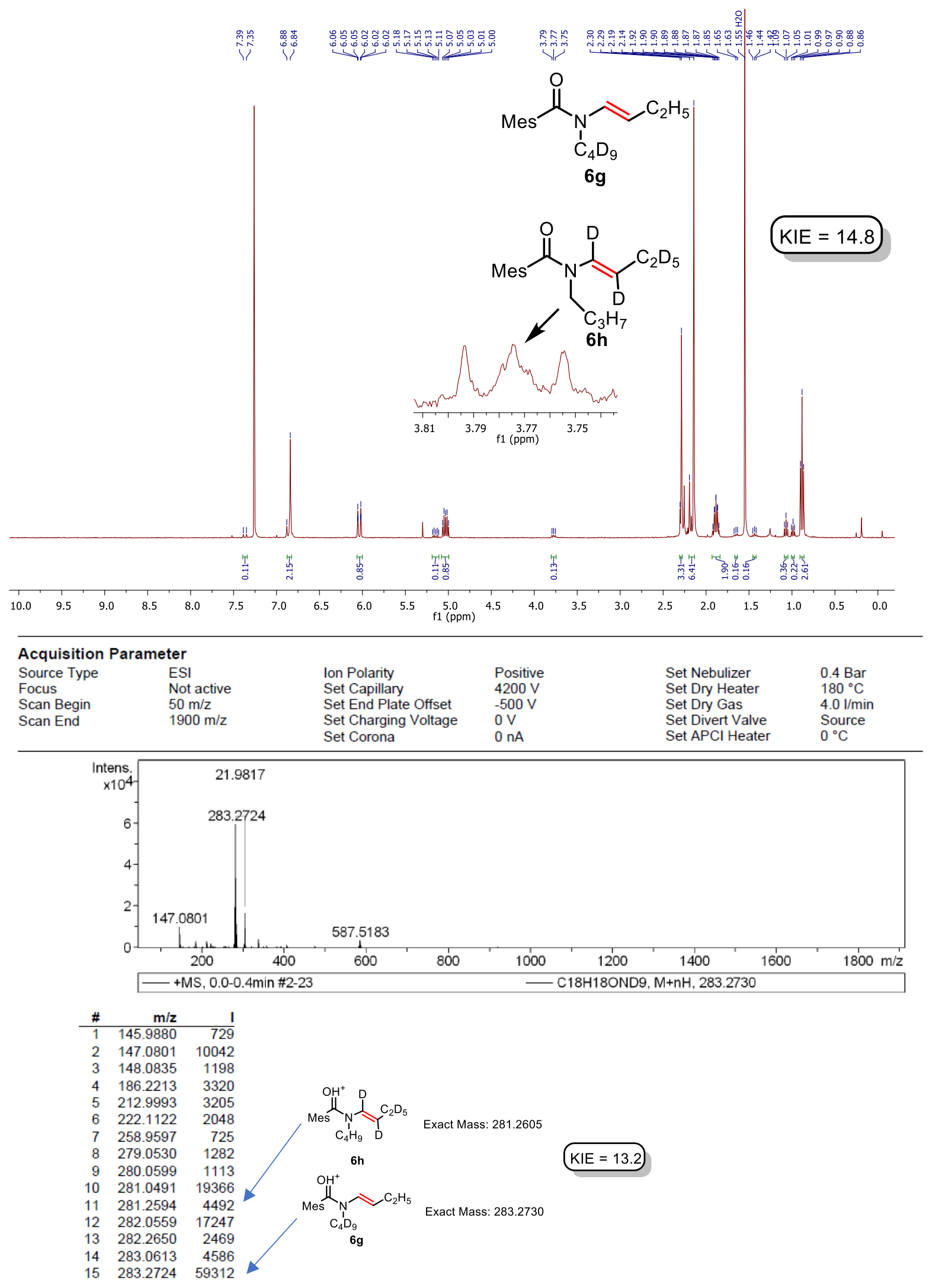




\subsection{Analysis of the Crude Material}<smiles>CC(=O)N1CCCCC1</smiles>

1a standard conditions

analysis of crude material
$2 \mathbf{a}$

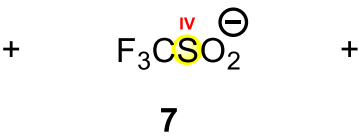

detected by ${ }^{19}$ F NMR

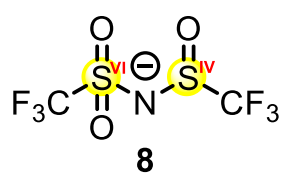

detected by HRMS

Following GP3 with substrate 1a, the obtained crude material was analyzed (without quenching with $\left.\mathrm{NH}_{4} \mathrm{Cl}\right)$ :

1) via ${ }^{19} \mathrm{~F}$ NMR spectroscopy ( $d^{6}$-DMSO was used as deuterated solvent). By the addition of 1 equivalent of Langlois salt $\left(\mathrm{CF}_{3} \mathrm{SO}_{2} \mathrm{Na}\right)$ which lead to an increase of signal a, the presence of $\mathbf{7}$ was proven. Additional experiments $\mathbf{A}$ and $\mathbf{B}$ were conducted to provide more insight of the additional fluorine species origin in the analyzed material.

${ }^{19} \mathrm{~F}$ NMR spectroscopy:
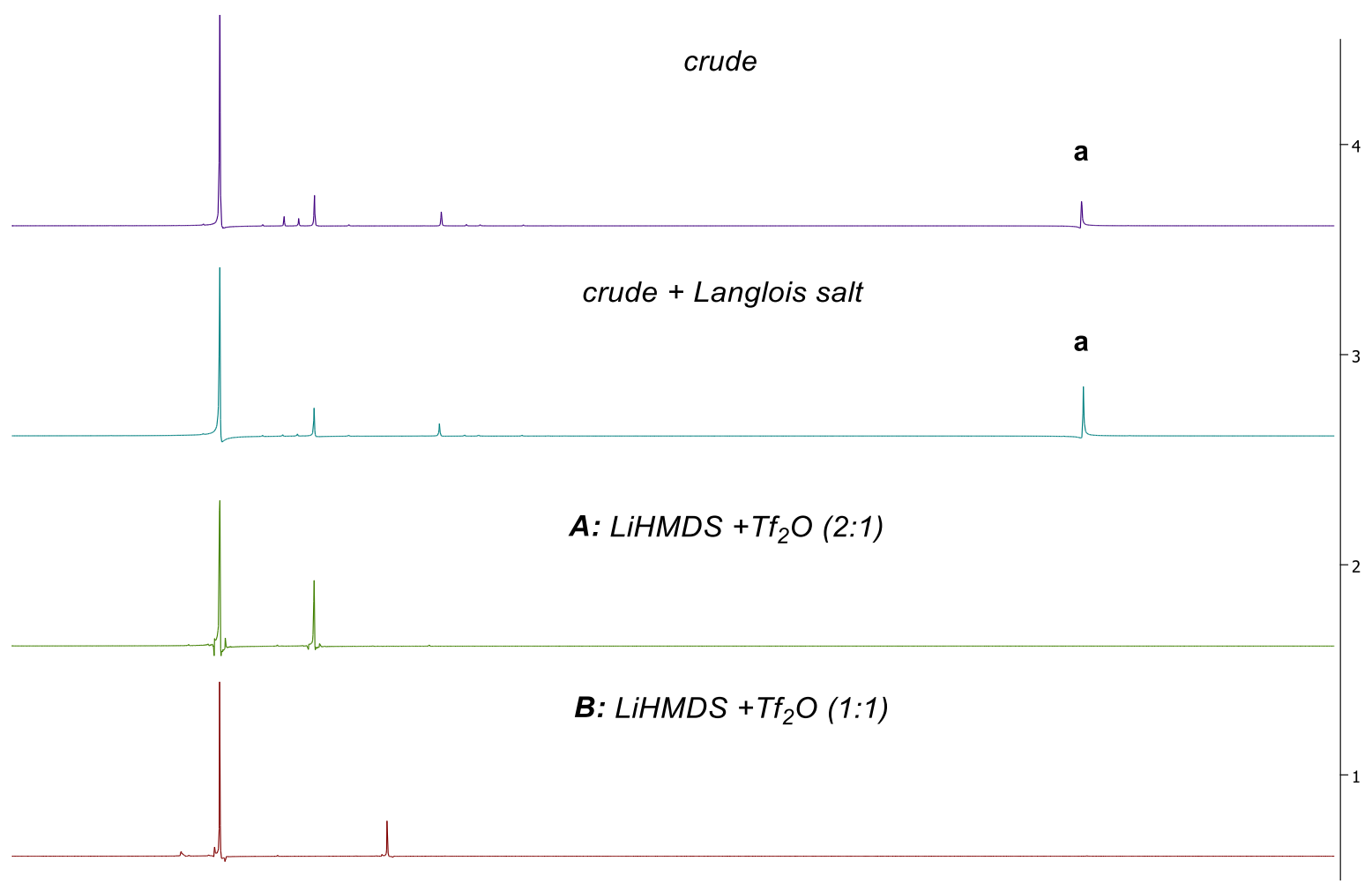

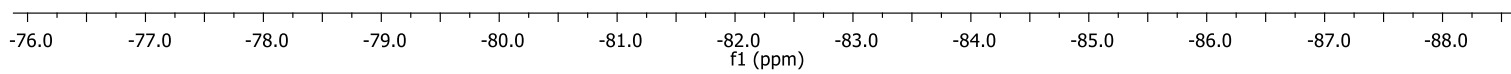


2) via HRMS

Species 8 was detected by HRMS:

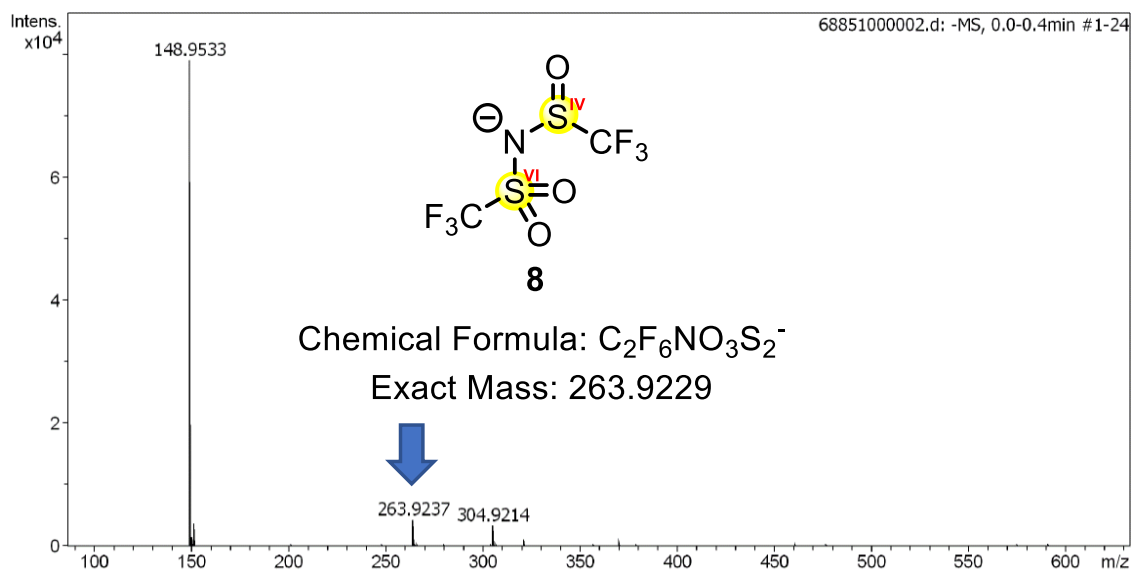

Proposed mechanism for the formation of 8:

A)<smiles>CN([As])[As]</smiles>

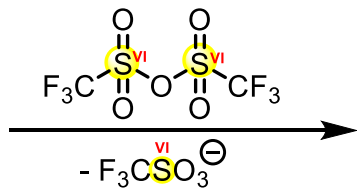

B)

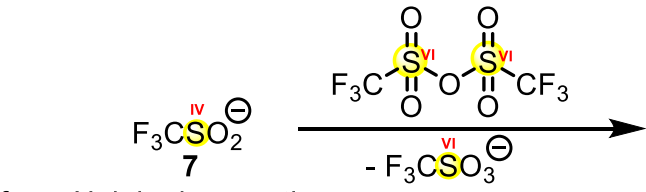
from $N$-dehydrogenation of the amide

C)<smiles>CS(=O)(=O)N([O-])S(=O)(=O)C(F)(F)F</smiles>

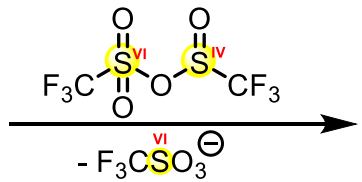<smiles>CN(S(C)(=O)=O)S(=O)(=O)N(C)S(=O)(=O)N(S(C)(=O)=O)S(=O)(=O)C(F)(F)F</smiles>

detected by GCMS<smiles>C[Si](=O)(F)(F)O[Si](=O)C(F)(F)F</smiles>
mixed S(IV)/S(VI) anhydride for some literature precedent on this reaction, see:

Tetrahedron Lett. 1996, 37, 8359-8362<smiles>CS(=O)(=O)N(S(=O)(=O)C(F)(F)F)[S+](=O)(=O)C(F)(F)F</smiles>

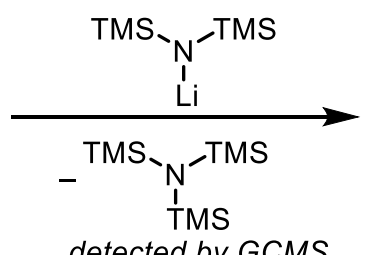<smiles>O=[S](=O)N([O-])S(=O)(=O)C(F)(F)F</smiles>

detected by GCMS see below

GCMS of worked-up crude (with substrate 1 a) showing the presence of $N(T M S)_{3}$ :

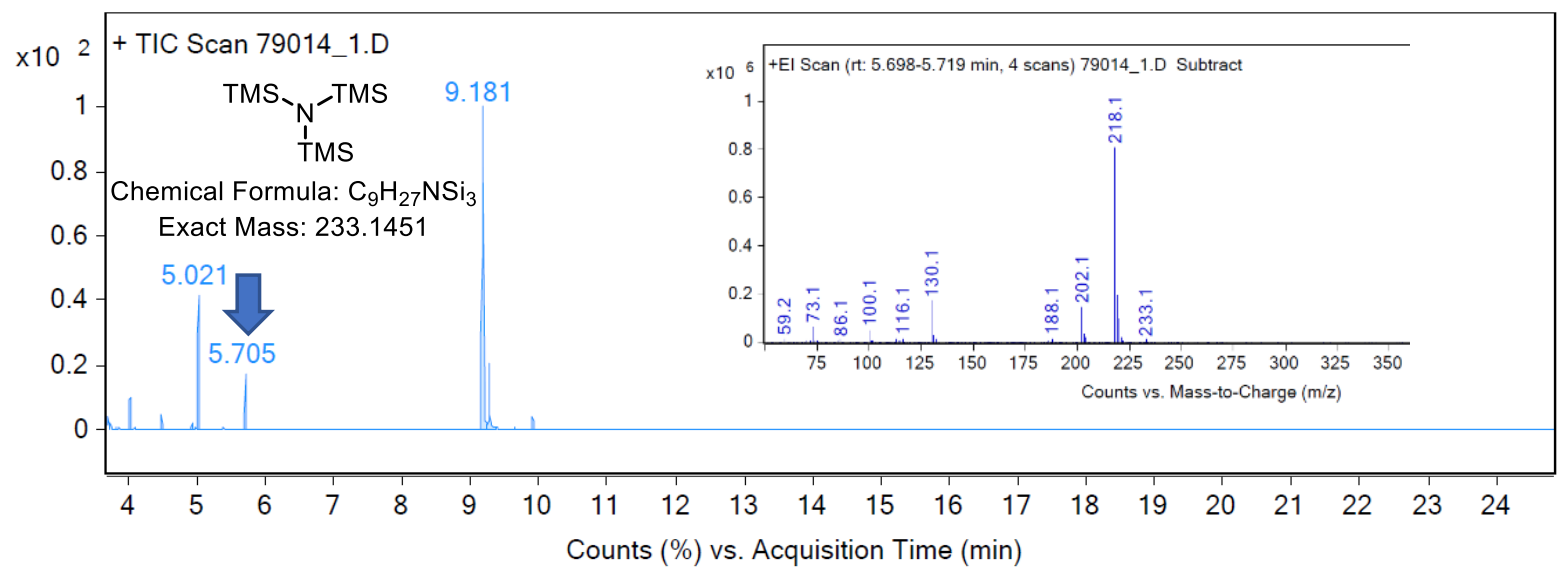




\subsection{Control Experiment}

Potential alternative pathway:

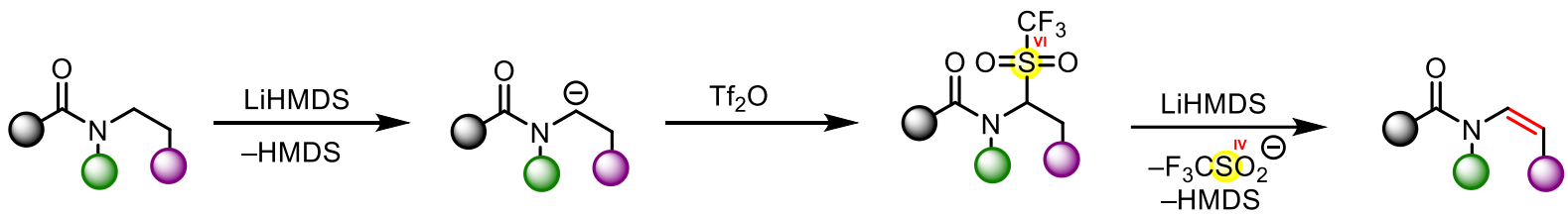

To test the possibility of the pathway shown above, following experiment was performed which did not reveal any deuterium incorporation:<smiles>Cc1cc(C)c(C(=O)N2CCCCC2)c(C)c1</smiles>

$1 \mathrm{a}$

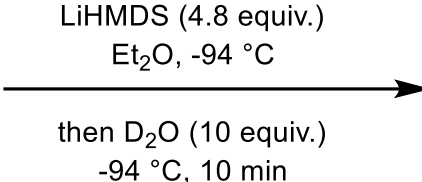

$-94{ }^{\circ} \mathrm{C}, 10 \mathrm{~min}$<smiles>[2H]C1CCCCN1C(=O)c1c(C)cc(C)cc1C</smiles>

not detected, only $1 \mathrm{a}$

In a flame-dried Schlenk flask, 1a $\left(69.4 \mathrm{mg}, 0.30 \mathrm{mmol}, 1.00\right.$ eq.) was dissolved in anhydrous $\mathrm{Et}_{2} \mathrm{O}$ (1.5 mL). The mixture was cooled down to $-94^{\circ} \mathrm{C}$ and LiHMDS ( $1 \mathrm{~m}$ in THF, $1.44 \mathrm{~mL}, 0.72 \mathrm{mmol}, 4.80$ eq., $1 \mathrm{M}$ in THF) was slowly added. The mixture was stirred for $10 \mathrm{~min}$, then $\mathrm{D}_{2} \mathrm{O}(54.1 \mu \mathrm{L}, 3.00 \mathrm{mmol}$, 10.0 eq.) was added in one portion. The reaction mixture was extracted with $\mathrm{CH}_{2} \mathrm{Cl}_{2}(3 \times 10 \mathrm{~mL})$, the organic phases were combined and dried over anhydrous $\mathrm{MgSO}_{4}$. After filtration and evaporation of solvent, the crude material was analyzed by NMR spectroscopy and mass spectrometry. No incorporation of deuterium was detected by both methods, instead pure 1a, with no signs of degradation, was recovered after extraction. Therefore, we exclude the possibility of the route shown above.

crude material (red spectra)

1a reference (blue spectra)

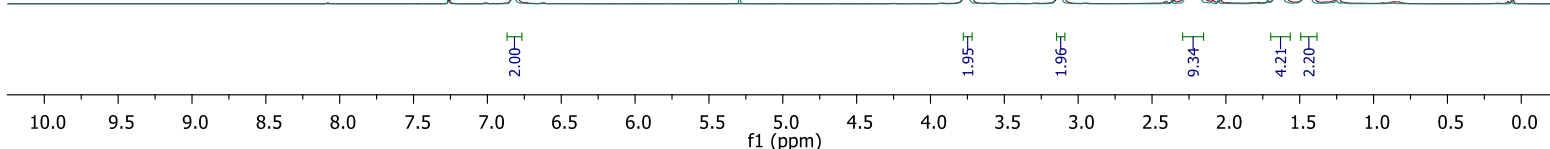




\section{References}

[1] T. E. Stang, P. J., Dueber, Org. Synth. 1974, 54, 79.

[2] T. Furukawa, M. Tobisu, N. Chatani, Bull. Chem. Soc. Jpn. 2016, 90, 332-342.

[3] M. Holik, A. Mannschreck, Org. Magn. Reson. 1979, 12, 223-228.

[4] J. Clayden, P. Johnson, J. H. Pink, J. Chem. Soc. Perkin Trans. 1 2001, 371-375.

[5] J. Karthikeyan, N. Yoshikai, Org. Lett. 2014, 16, 4224-4227.

[6] W. Huang, M.-L. Xu, J. Chem. Res. 2013, 37, 77-79.

[7] G.-P. Yang, K. Li, W. Liu, K. Zeng, Y.-F. Liu, Org. Biomol. Chem. 2020, 18, 6958-6964.

[8] Z. Yin, Z. Wang, X.-F. Wu, European J. Org. Chem. 2017, 2017, 3992-3995.

[9] N. Sharma, G. Sekar, Adv. Synth. Catal. 2016, 358, 314-320.

[10] G. Pandey, S. Koley, R. Talukdar, P. K. Sahani, Org. Lett. 2018, 20, 5861-5865.

[11] S. Srinivas Kotha, S. Badigenchala, G. Sekar, Adv. Synth. Catal. 2015, 357, 1437-1445.

[12] W. Li, X.-F. Wu, Org. Lett. 2015, 17, 1910-1913.

[13] X. Wu, W. Fan, Y. Pan, Y. Zhai, Y. Niu, C. Li, Q. Mei, Molecules 2014, 19, 1034-1046.

[14] J. Li, F. Xu, Y. Zhang, Q. Shen, J. Org. Chem. 2009, 74, 2575-2577.

[15] D.-Y. Huang, Q.-J. Yao, S. Zhang, X.-T. Xu, K. Zhang, B.-F. Shi, Org. Lett. 2019, 21, 951-954.

[16] Y.-S. Bao, M. Baiyin, B. Agula, M. Jia, B. Zhaorigetu, J. Org. Chem. 2014, 79, 6715-6719.

[17] N. Gigant, L. Chausset-Boissarie, M.-C. Belhomme, T. Poisson, X. Pannecoucke, I. Gillaizeau, Org. Lett. 2013, 15, 278-281.

[18] M. Feng, X. Jiang, Chem. Commun. 2014, 50, 9690-9692.

[19] O. Di Pietro, E. Viayna, E. Vicente-García, M. Bartolini, R. Ramón, J. Juárez-Jiménez, M. V. Clos, B. Pérez, V. Andrisano, F. J. Luque, R. Lavilla, D. M. Muñoz-Torrero, Eur. J. Med. Chem. 2014, 73, 141-152.

[20] J. B. Feltenberger, R. Hayashi, Y. Tang, E. S. C. Babiash, R. P. Hsung, Org. Lett. 2009, 11, 36663669.

[21] W. Marais, C. W. Holzapfel, Synth. Commun. 1998, 28, 3681-3691.

[22] J. B. Roque, Y. Kuroda, L. T. Göttemann, R. Sarpong, Science 2018, 361, 171-174.

[23] N. Gigant, L. Chausset-Boissarie, M.-C. Belhomme, T. Poisson, X. Pannecoucke, I. Gillaizeau, Org. Lett. 2013, 15, 278-281.

[24] R. Ito, N. Umezawa, T. Higuchi, J. Am. Chem. Soc. 2005, 127, 834-835. 


\section{NMR Spectra}

Azepan-1-yl(mesityl)methanone (1c)

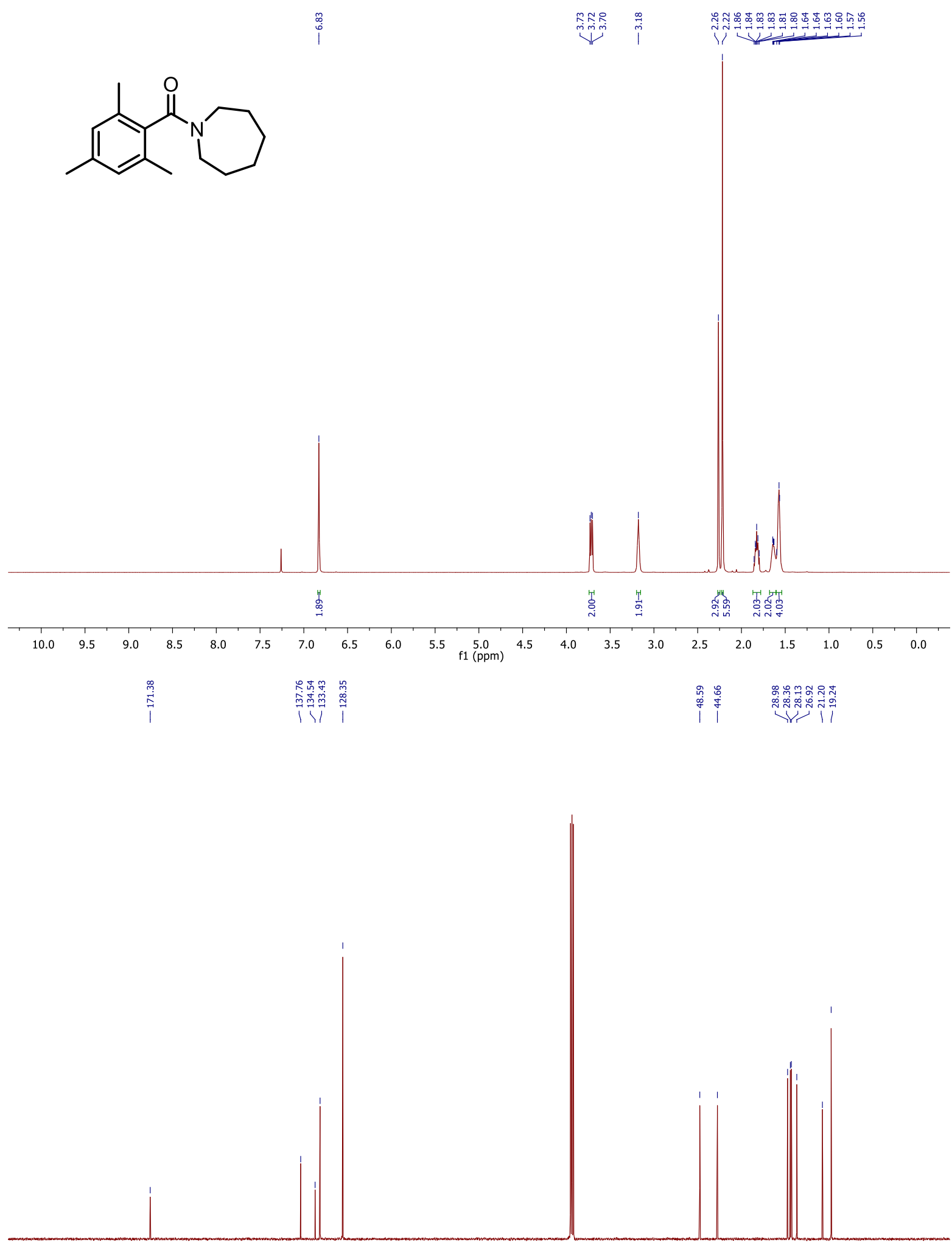

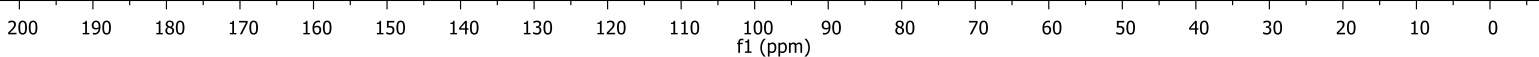


Mesityl(1,4-dioxa-8-azaspiro[4.5]decan-8-yl)methanone (1d)

$\stackrel{\infty}{i}$

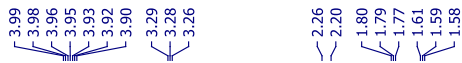<smiles>Cc1cc(C)c(C(=O)N2CCC3(CC2)OCCO3)c(C)c1</smiles>
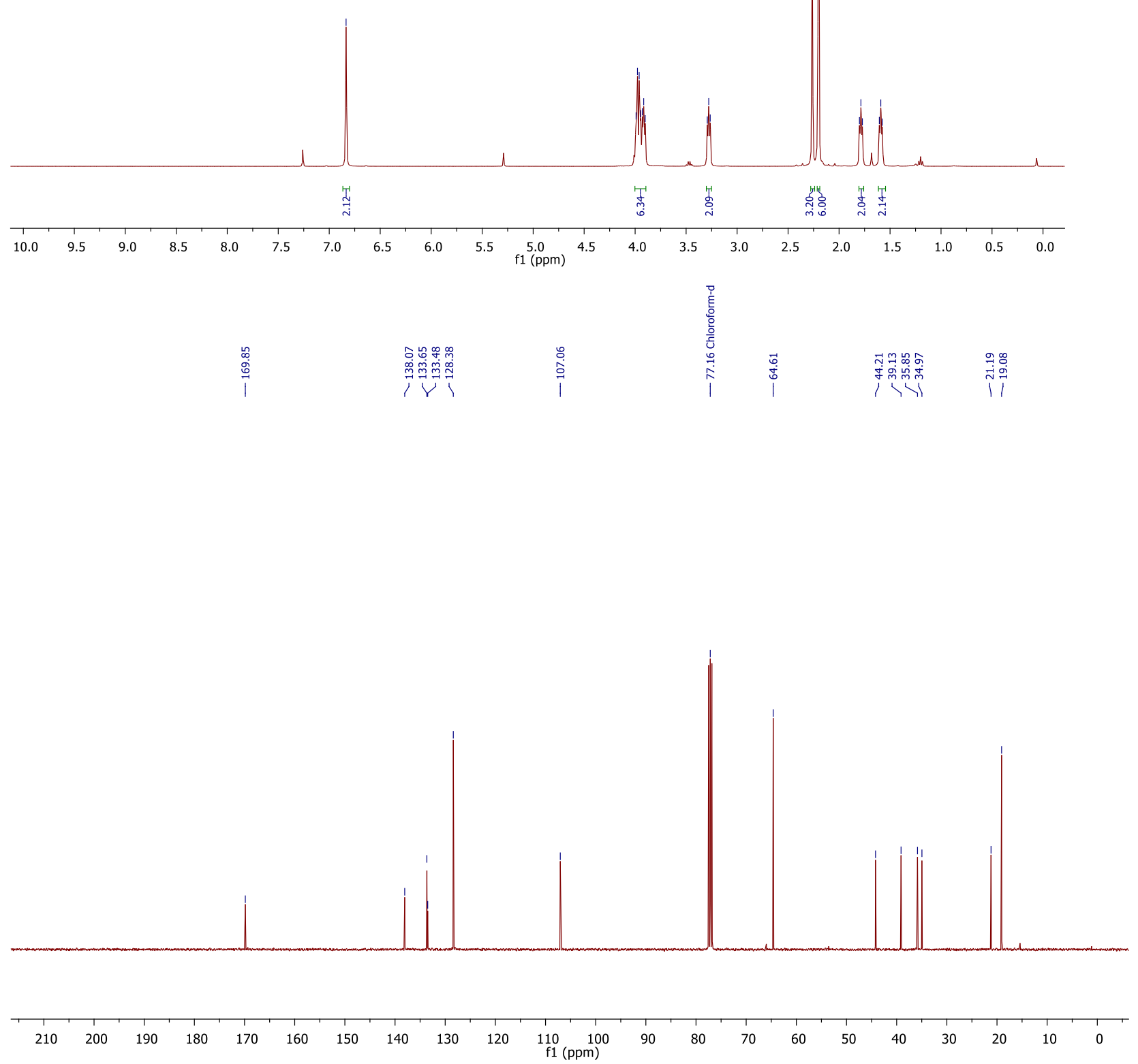
Mesityl(octahydro-2H-isoindol-2-yl)methanone (1e)

总

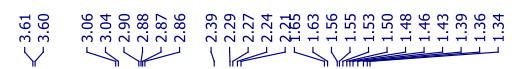
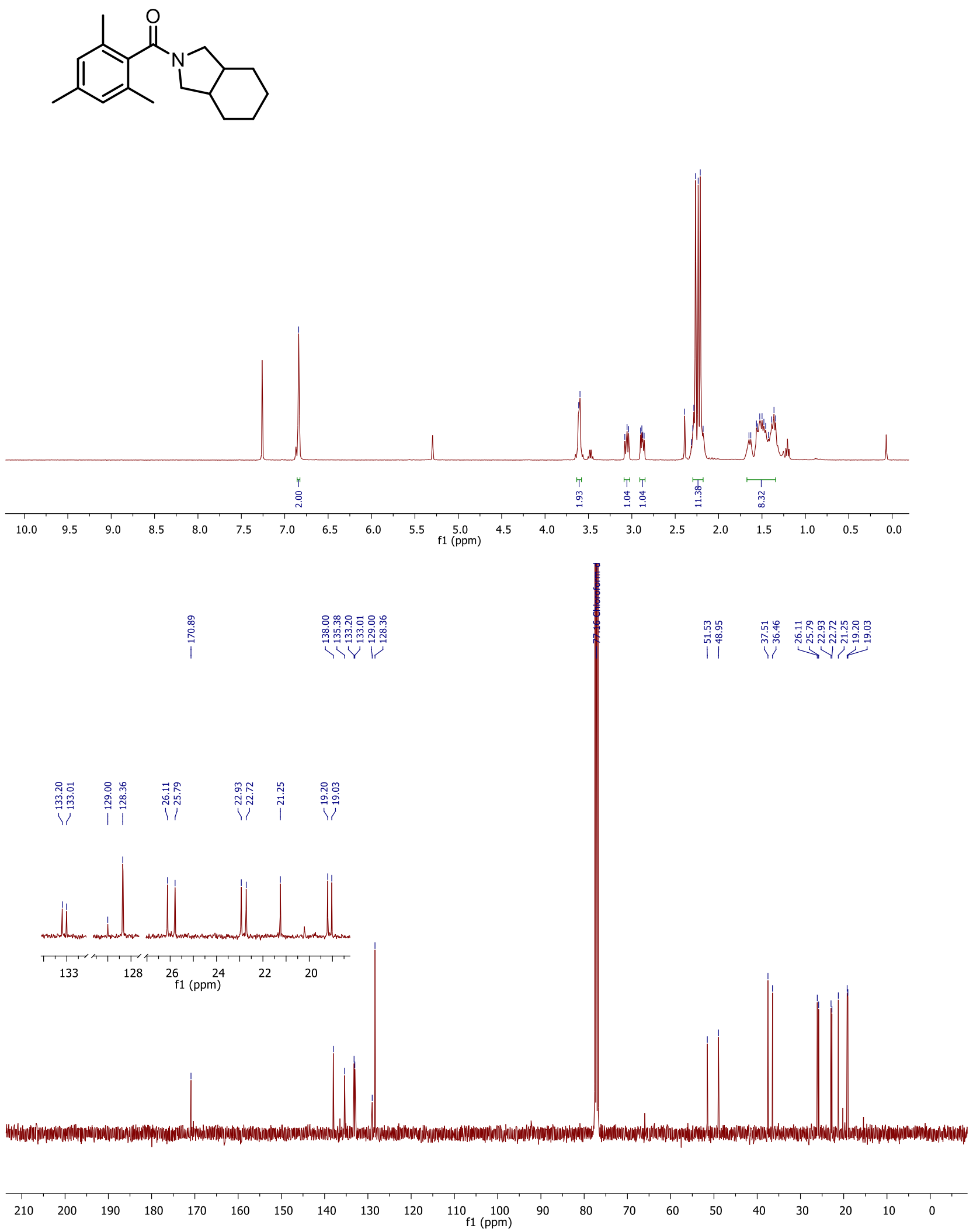

47 
Mesityl(morpholino)methanone (1f)

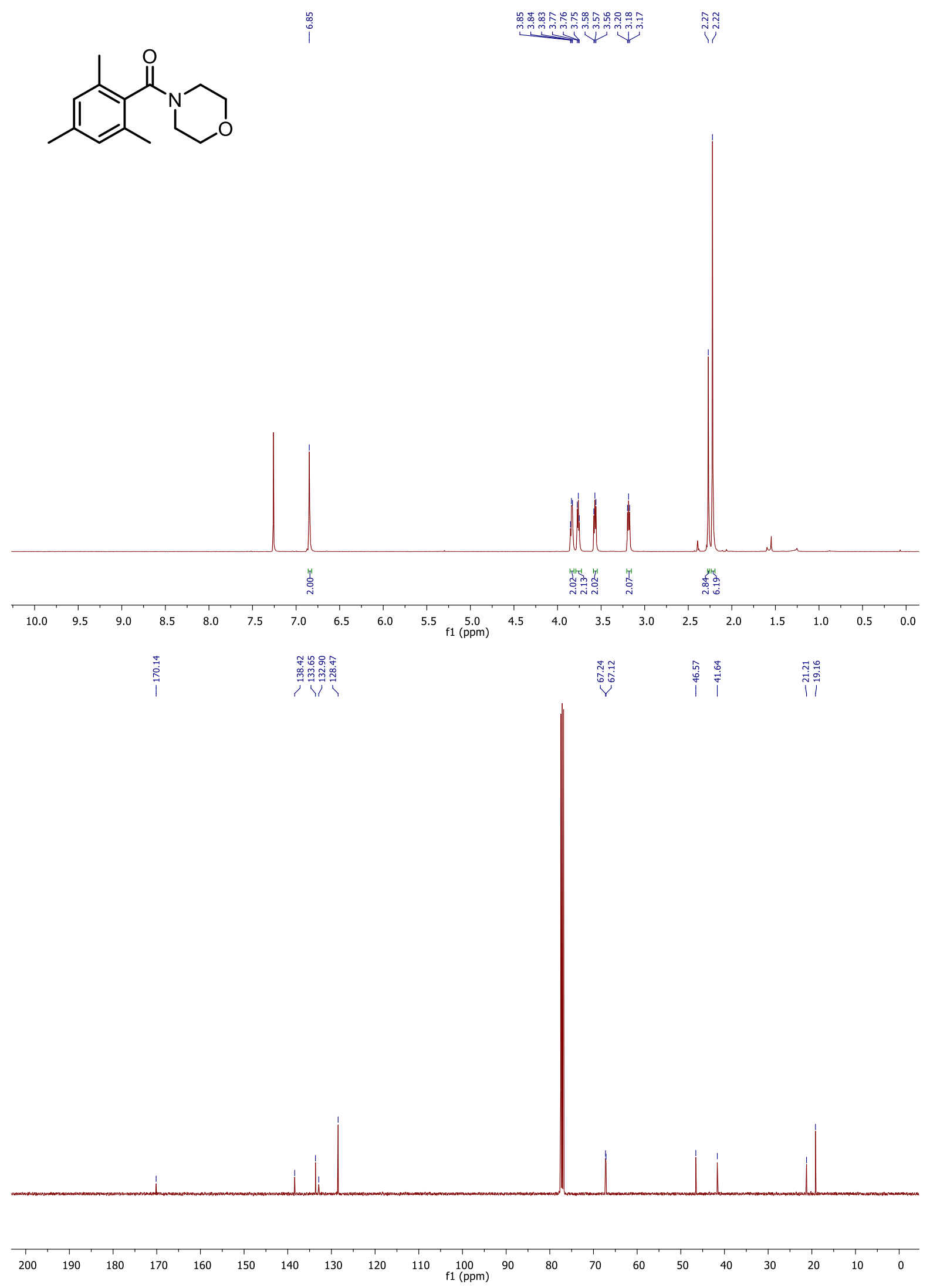


Ethyl 4-(2,4,6-trimethylbenzoyl)-3,4-dihydropyrazine-1(2H)-carboxylate (1g)

i

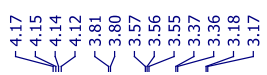

กิ่

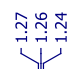
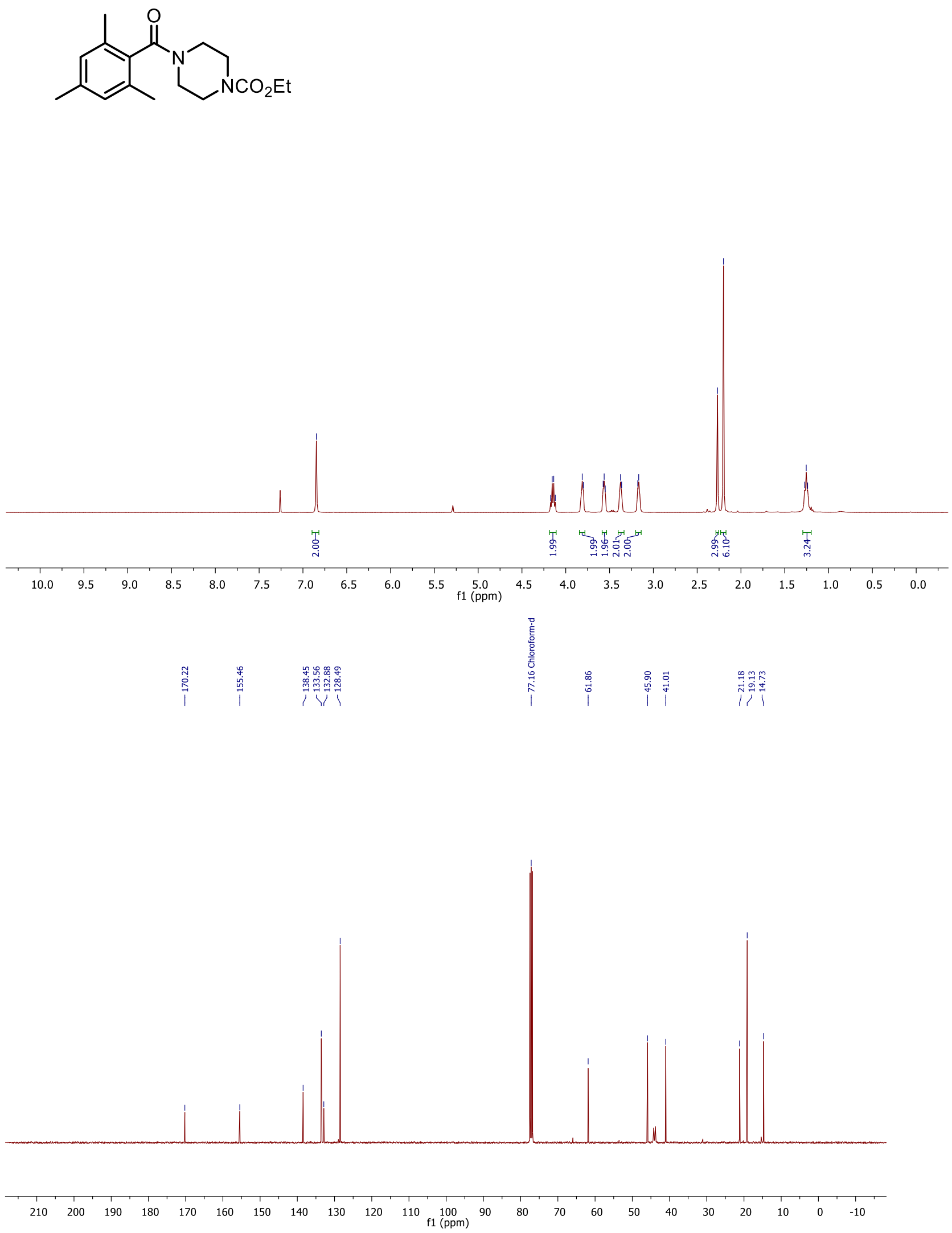

49 
2,4,6-Trimethyl-N,N-dipropylbenzamide (1i)

œ

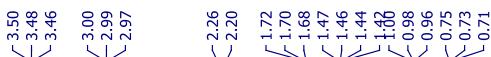<smiles>CCCN(CCC)C(=O)c1c(C)cc(C)cc1C</smiles>
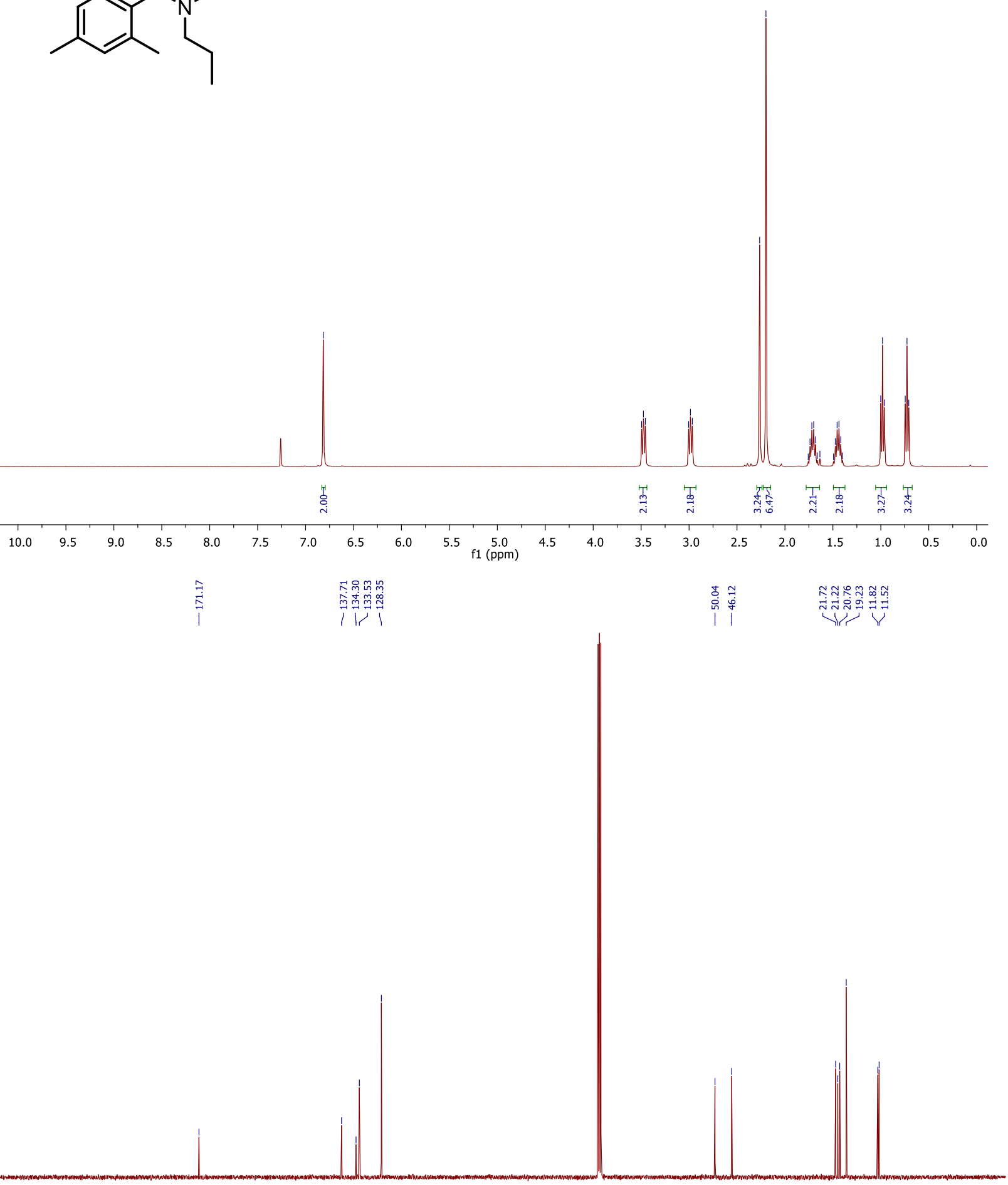

$\begin{array}{lllllllllllllllllllllllllll}210 & 200 & 190 & 180 & 170 & 160 & 150 & 140 & 130 & 120 & 110 & 100 & 90 & 80 & 70 & 60 & 50 & 40 & 30 & 20 & 10 & 0 & -10\end{array}$ 
N,N-Dibutyl-2,4,6-trimethylbenzamide (1j)

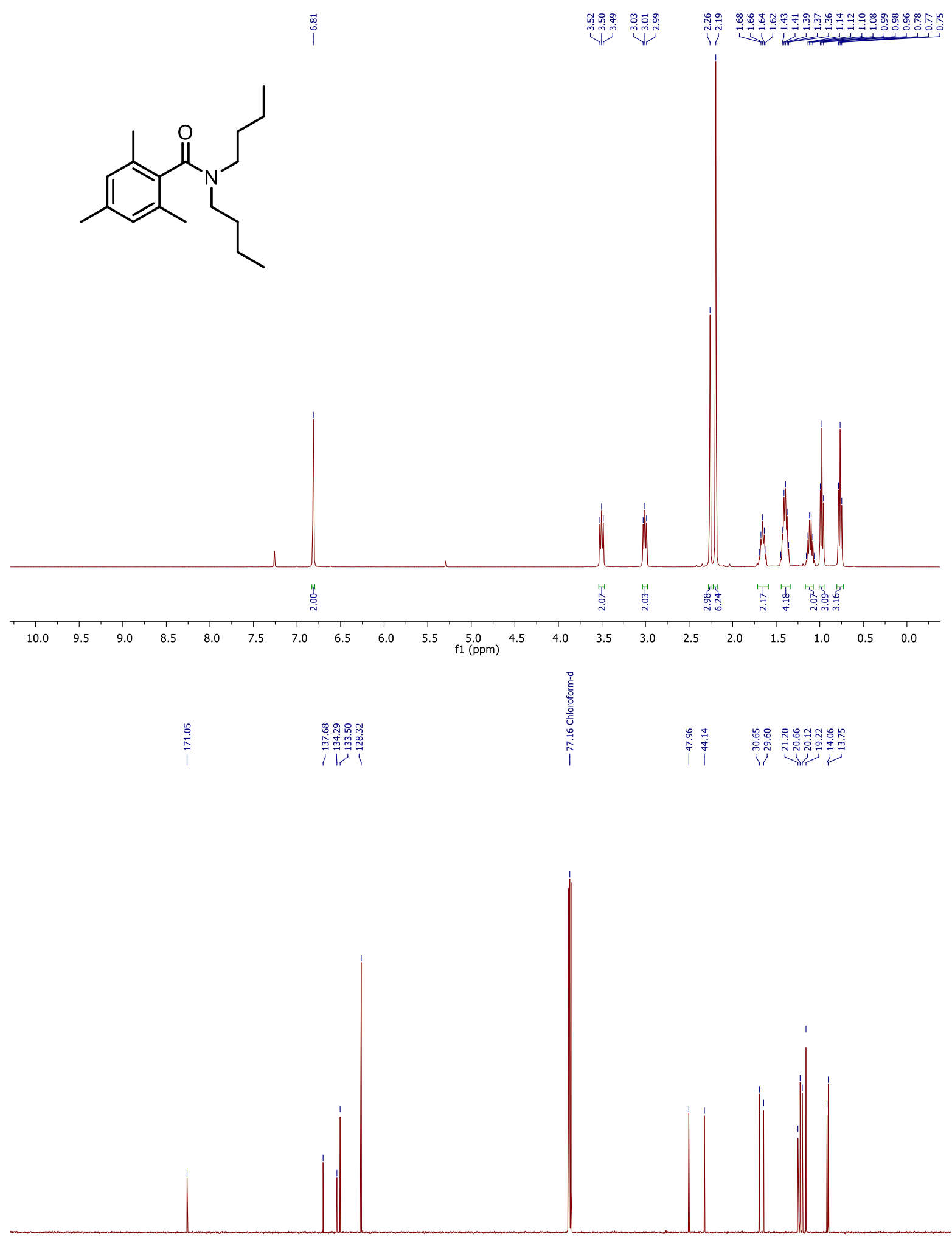

$\begin{array}{lllllllllllllllllllllllllllll}210 & 200 & 190 & 180 & 170 & 160 & 150 & 140 & 130 & 120 & 110 & 100 & 90 & 80 & 70 & 60 & 50 & 40 & 30 & 20 & 10 & 0 & -10\end{array}$ 


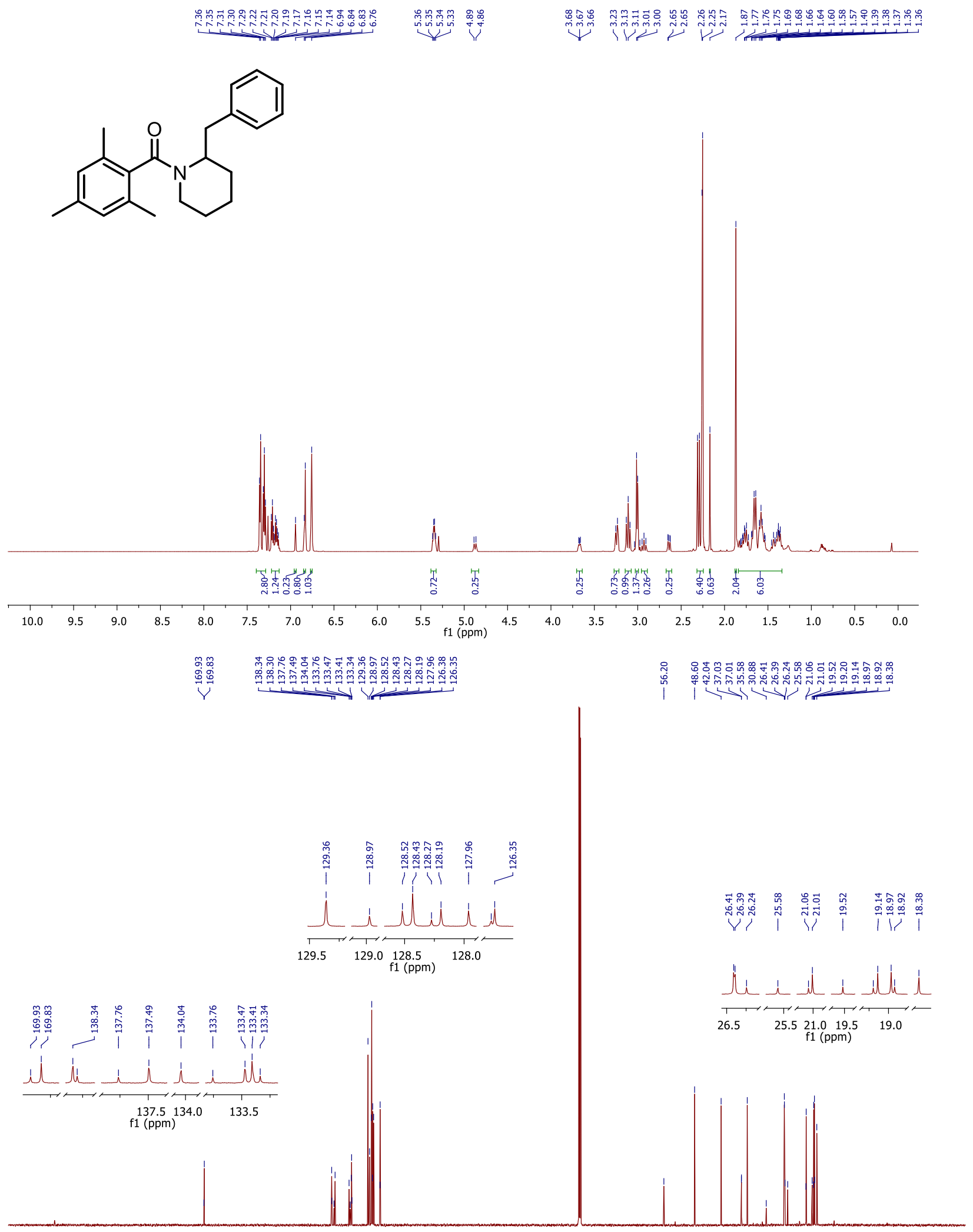

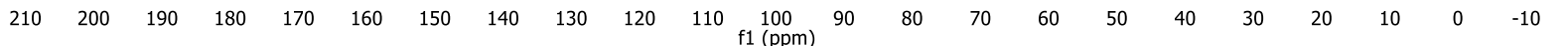


Mesityl(2-methylpiperidin-1-yl)methanone (1l)

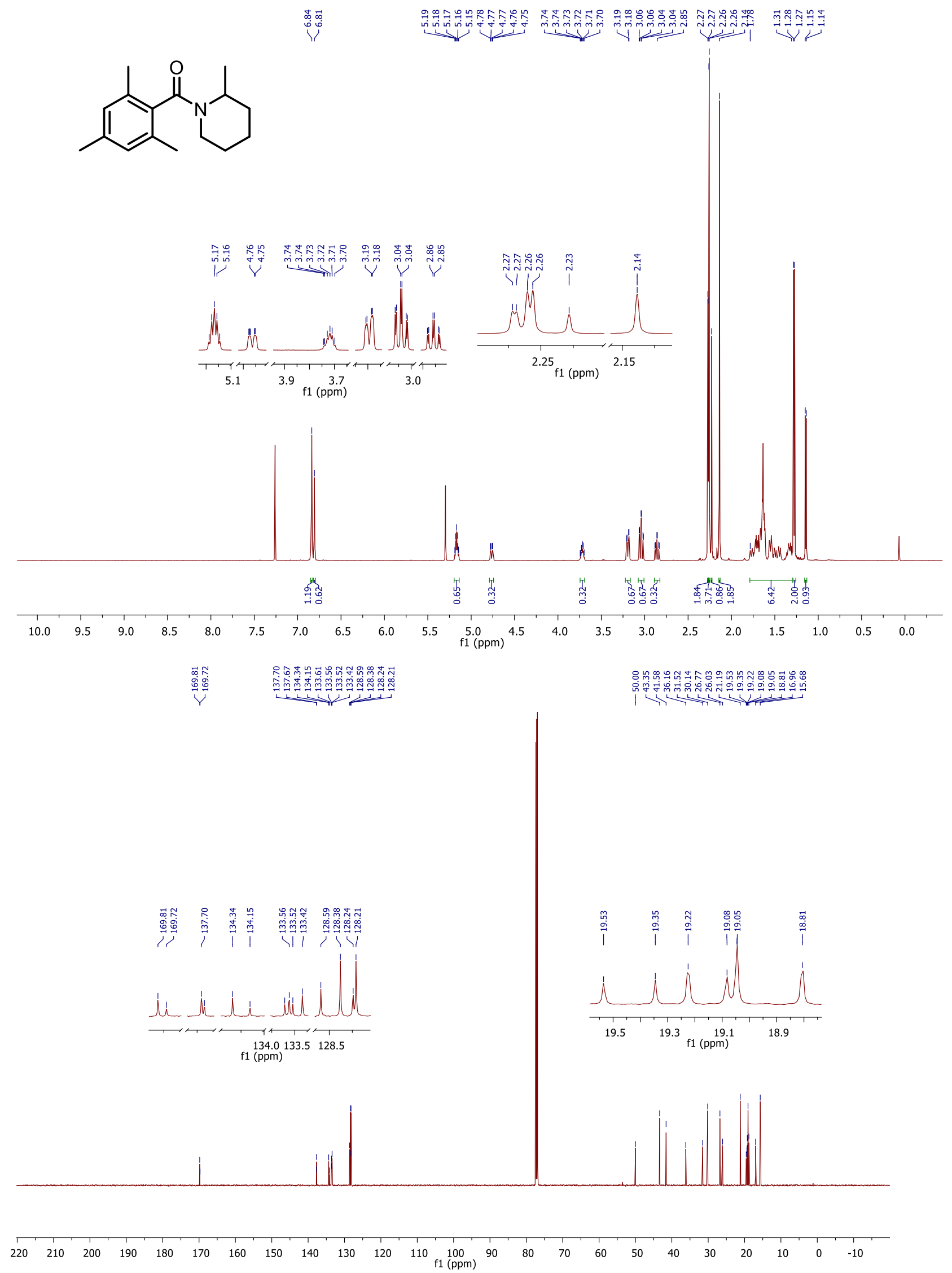



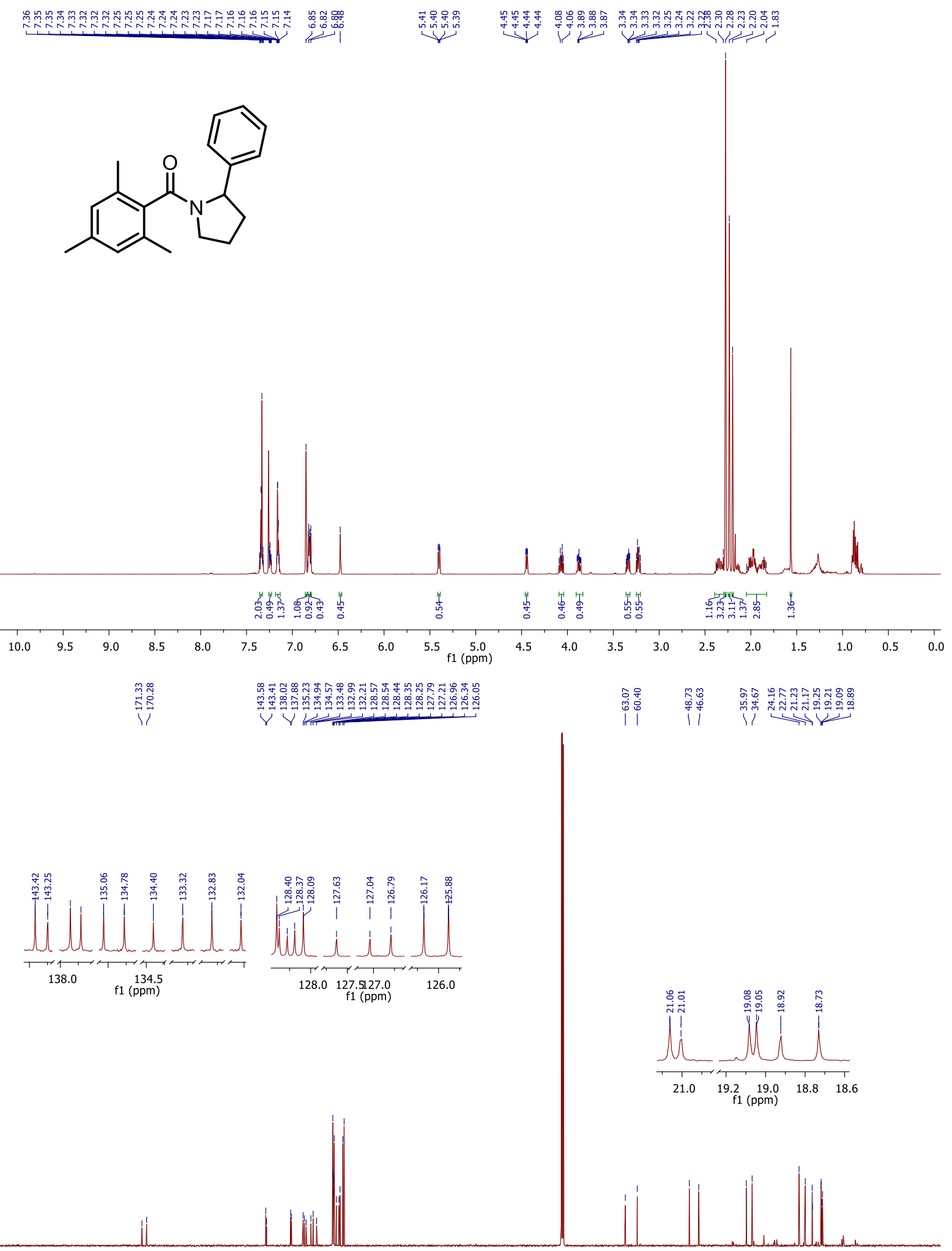

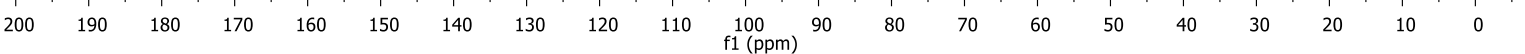




\section{Mesityl((4aS,8aR)-octahydroquinolin-1(2H)-yl)methanone (1n)}

\i:

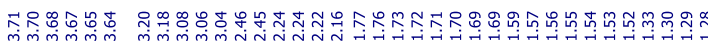<smiles>Cc1cc(C)c(C(=O)N2CCC[C@H]3CCCC[C@]32C)c(C)c1</smiles>

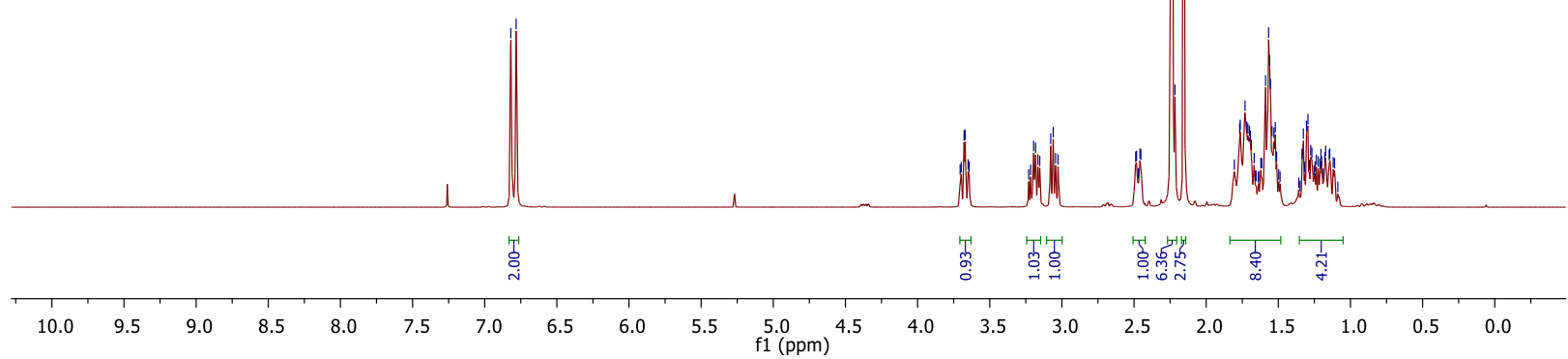

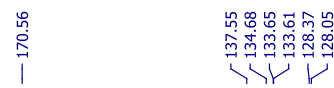

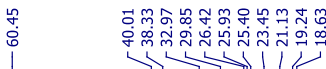

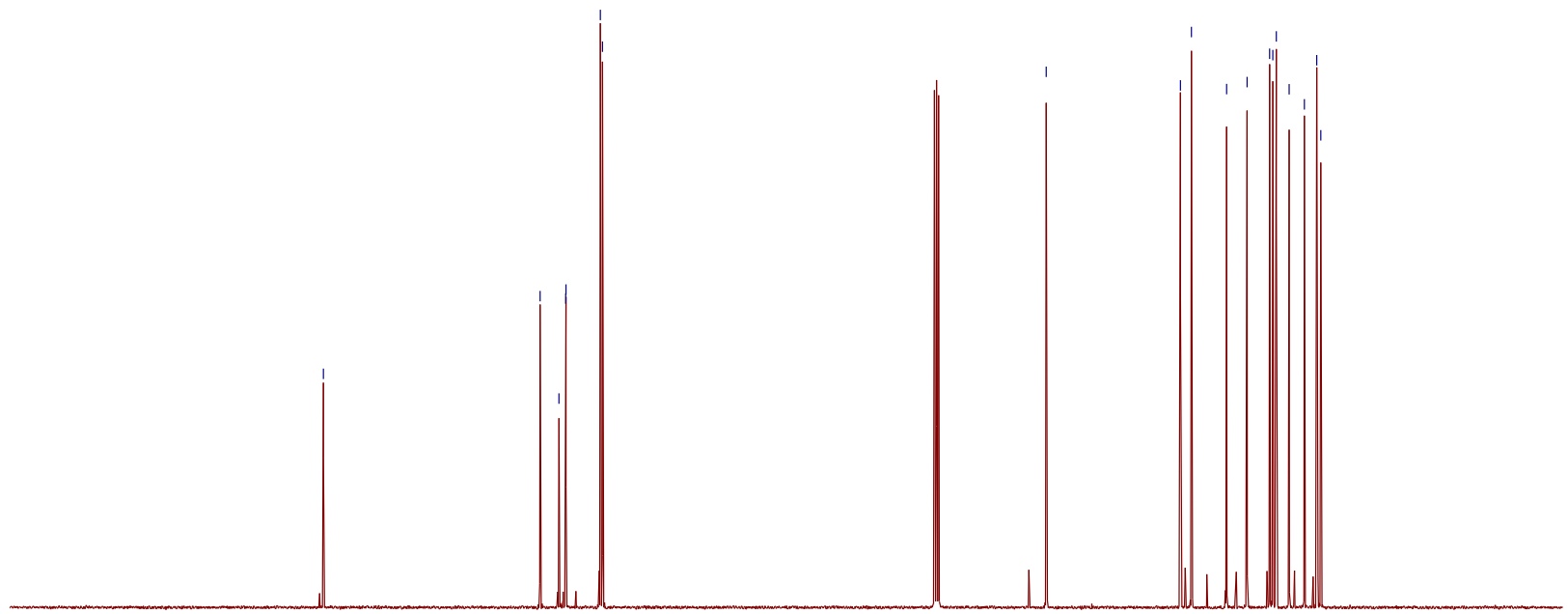

$\begin{array}{llllllllllllllllllllllll}210 & 200 & 190 & 180 & 170 & 160 & 150 & 140 & 130 & 120 & 110 & 100 & 90 & 80 & 70 & 60 & 50 & 40 & 30 & 20 & 10 & 0 & -10\end{array}$ 
$\mathrm{N}$-Cyclohexyl-N-ethyl-2,4,6-trimethylbenzamide (10)

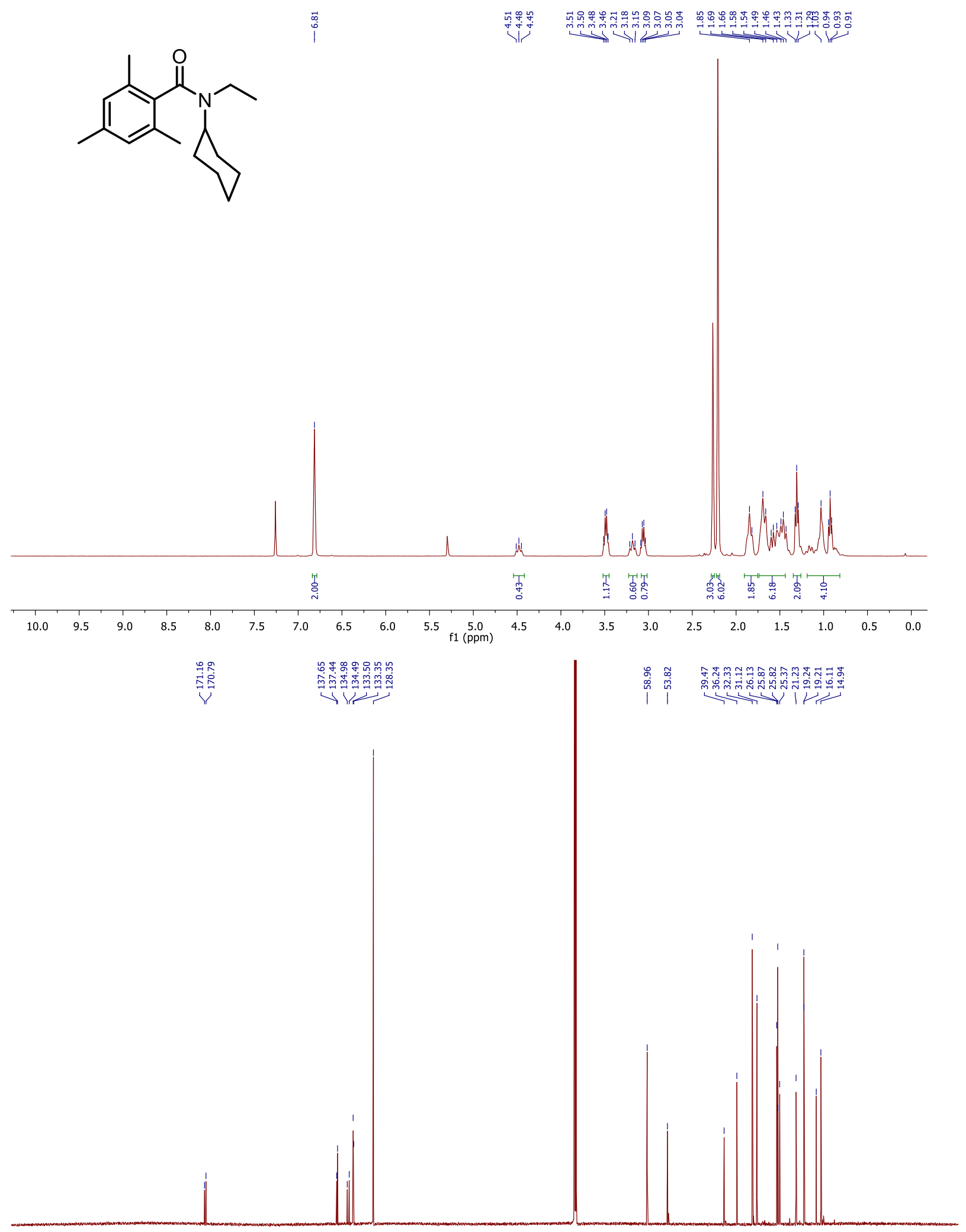

$\begin{array}{rlllllllllllllllllllllll}220 & 210 & 200 & 190 & 180 & 170 & 160 & 150 & 140 & 130 & 120 & 110 & 100 & 100 & 80 & 70 & 60 & 50 & 40 & 30 & 20 & 10 & 0 & -10\end{array}$ 
N-Butyl-N-ethyl-2,4,6-trimethylbenzamide (1p)

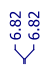

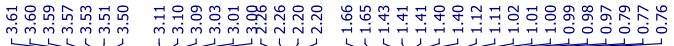<smiles>CCCCN(CC)C(=O)c1c(C)cc(C)cc1C</smiles>
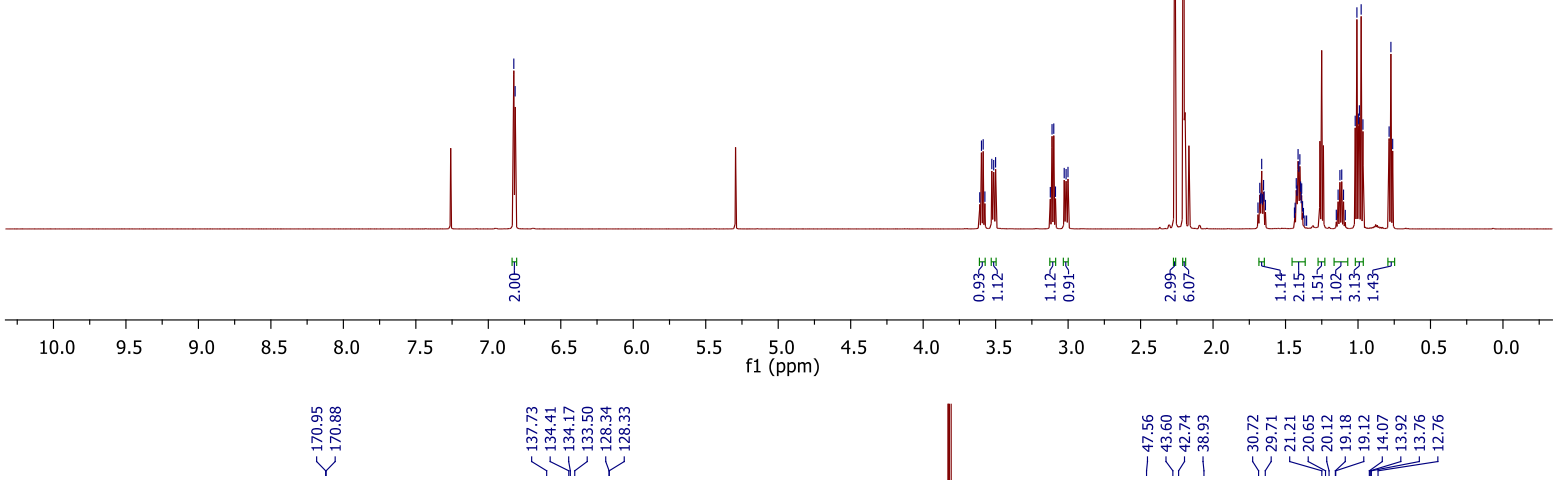

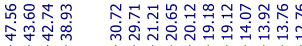

1)

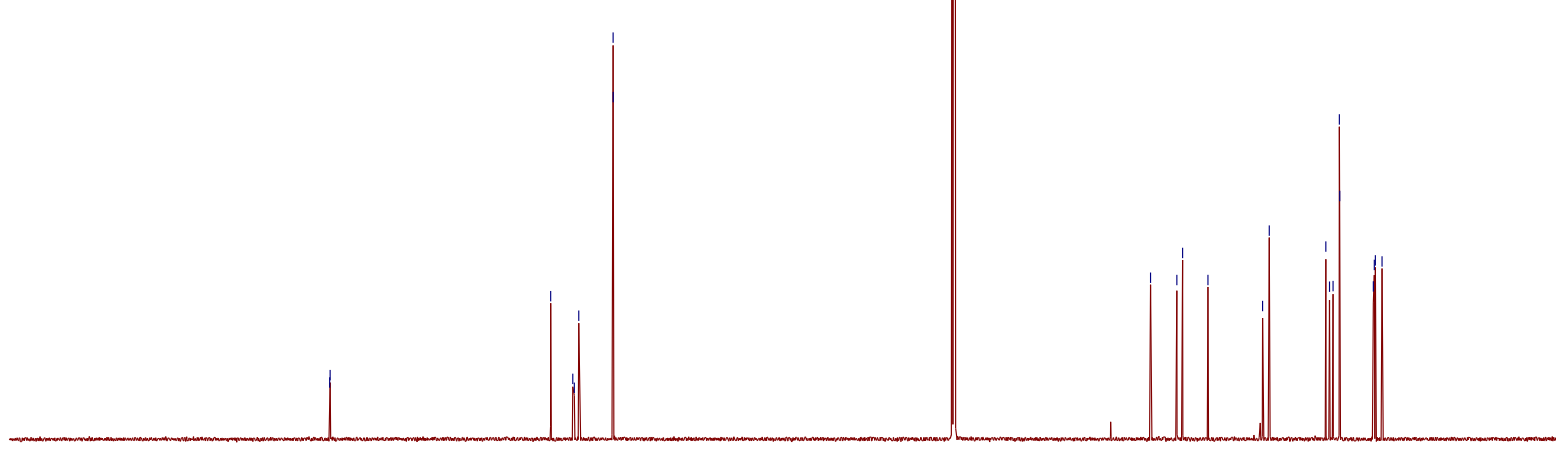

$\begin{array}{lllllllllllllllllllllll}210 & 200 & 190 & 180 & 170 & 160 & 150 & 140 & 130 & 120 & 110 & 100 & 90 & 80 & 70 & 60 & 50 & 40 & 30 & 20 & 10 & 0 & -10\end{array}$ 
((3S,4R)-3-((Benzo[d][1,3]dioxol-5-yloxy)methyl)-4-(4-fluorophenyl)piperidin-1yl)(mesityl)methanone (1q)

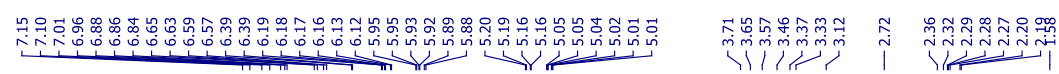

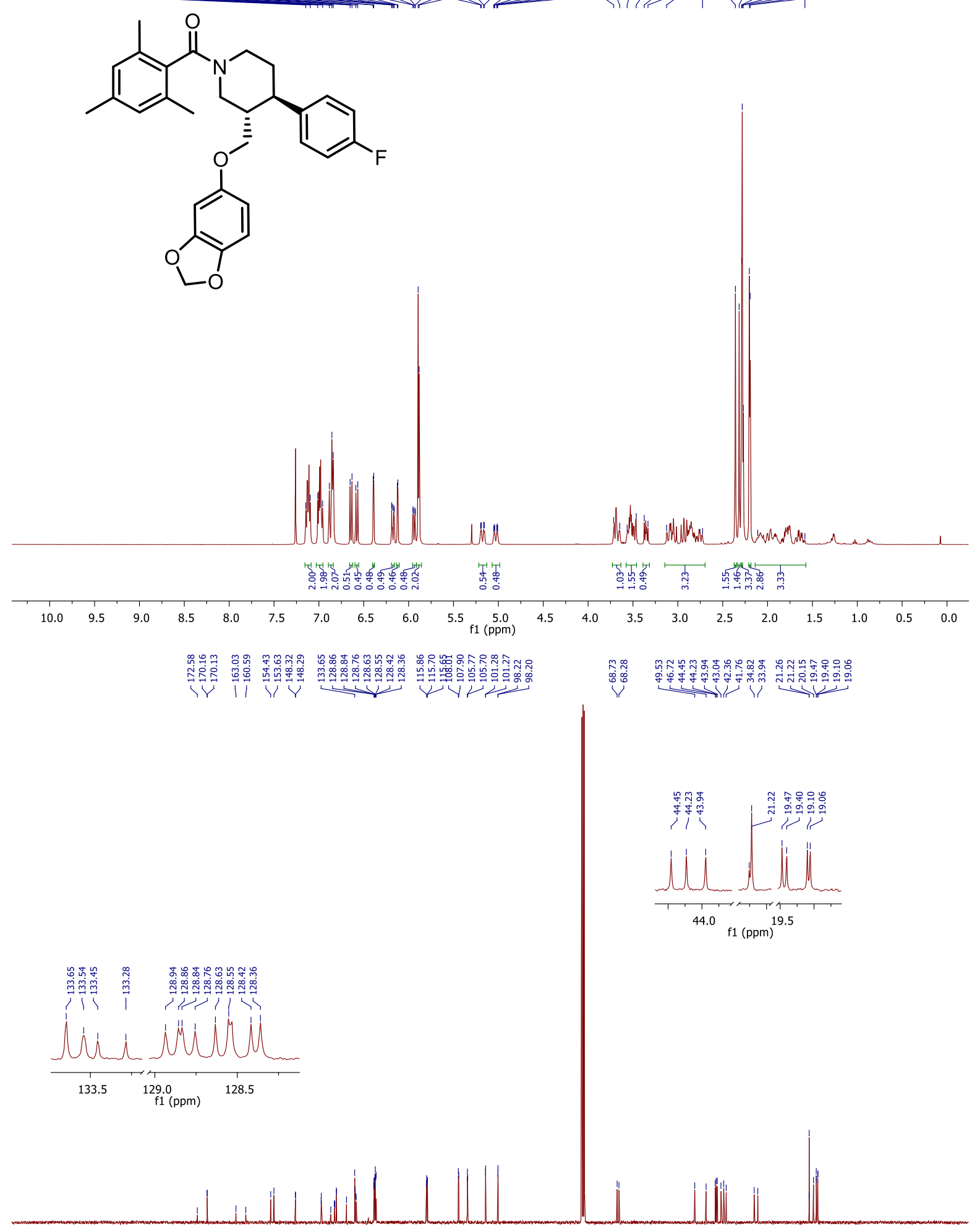

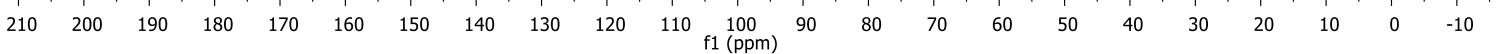




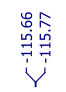

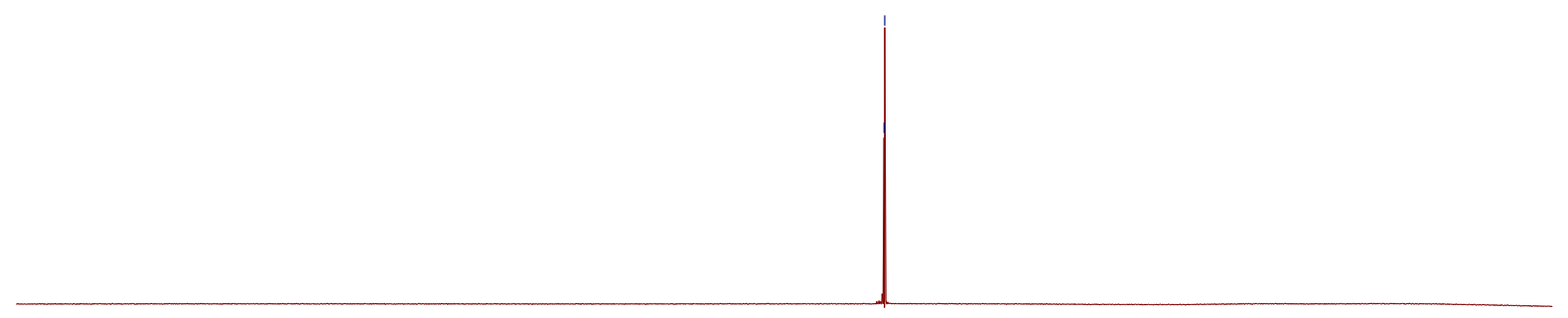

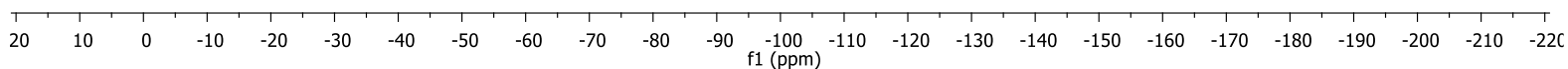




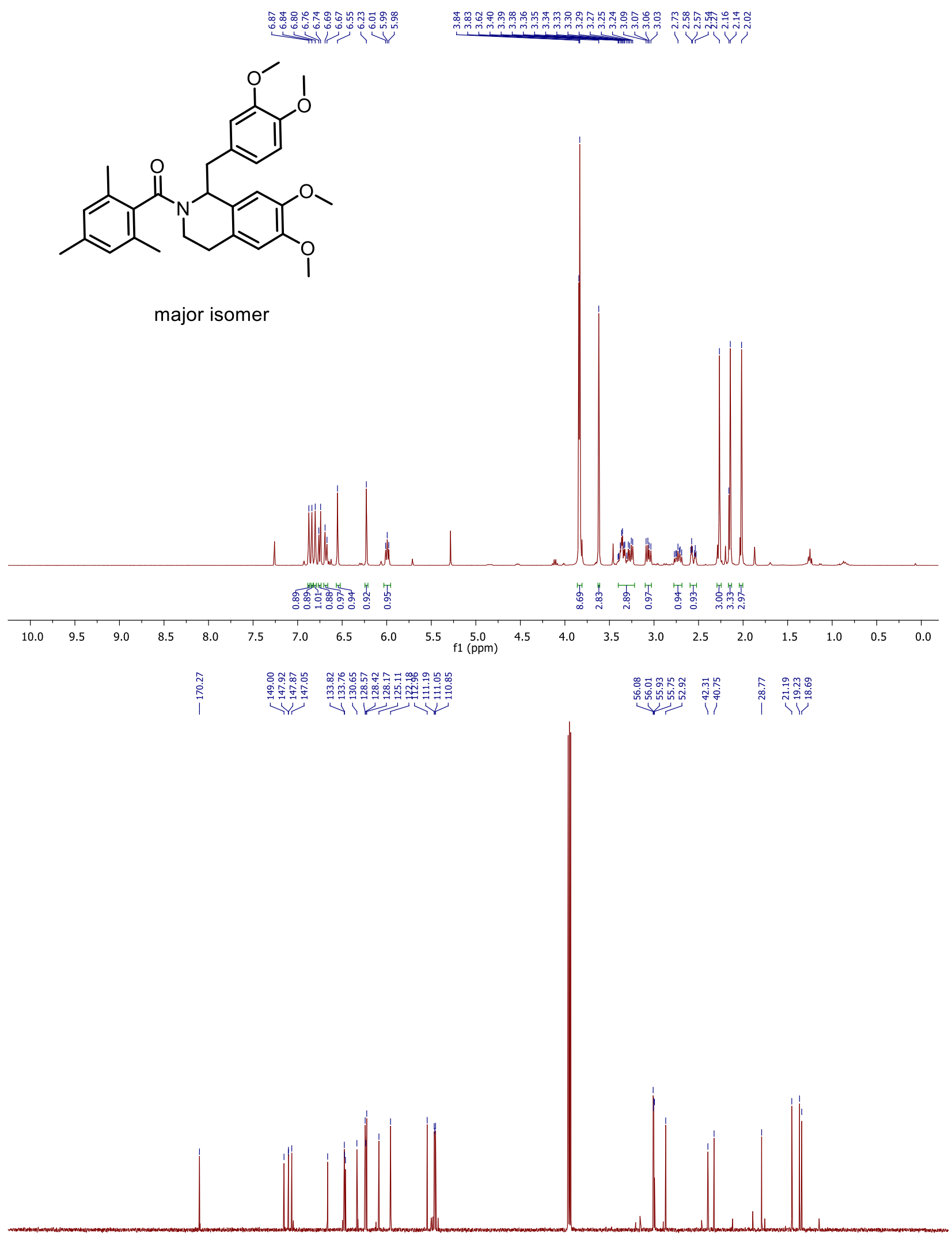

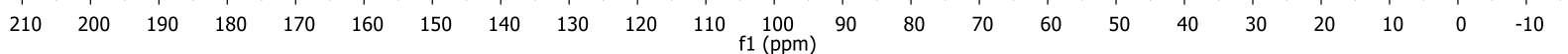




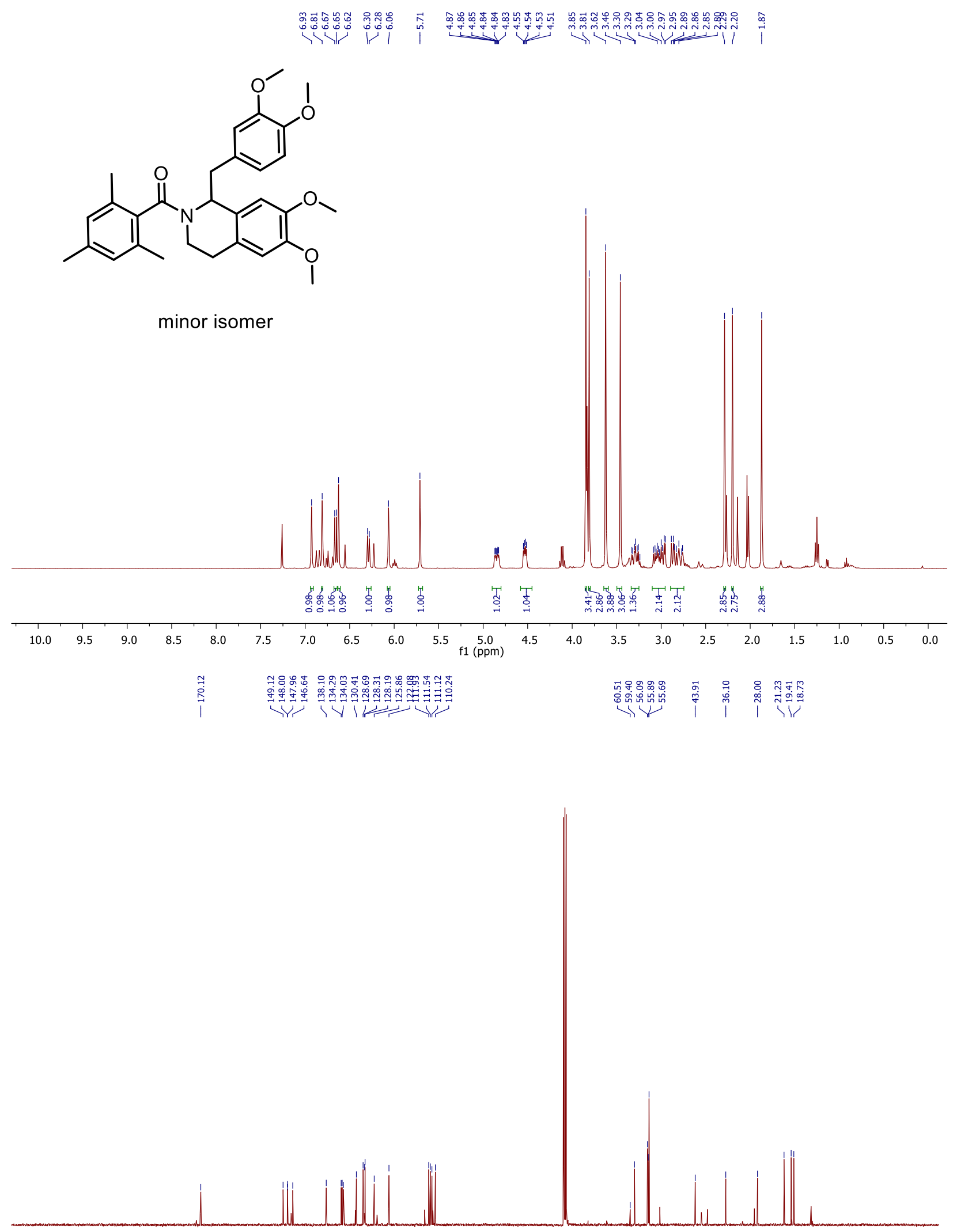

$\begin{array}{lllllllllllllllllllllll}210 & 200 & 190 & 180 & 170 & 160 & 150 & 140 & 130 & 120 & 110 & \underset{\mathrm{f} 1}{1(\mathrm{ppm})} & 90 & 80 & 70 & 60 & 50 & 40 & 30 & 20 & 10 & 0 & -10\end{array}$ 
Pyrrolidin-1-yl(2,4,6-triisopropylphenyl)methanone (3a)

i

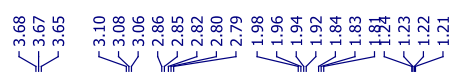
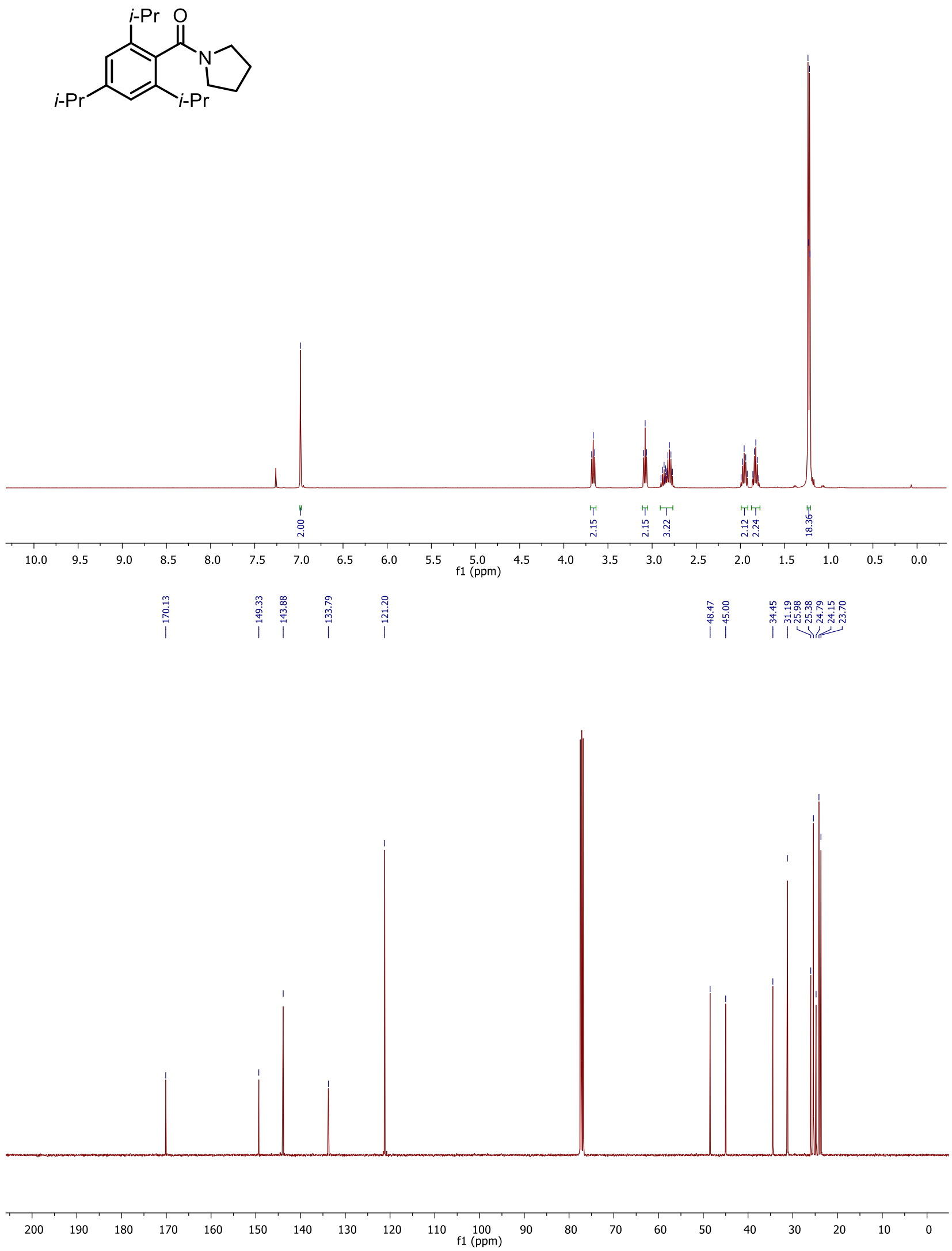

62 
Pyrrolidin-1-yl(3,4,5-trimethoxyphenyl)methanone (3j)<smiles>COc1cc(C(=O)N2CCCC2)cc(OC)c1OC</smiles>
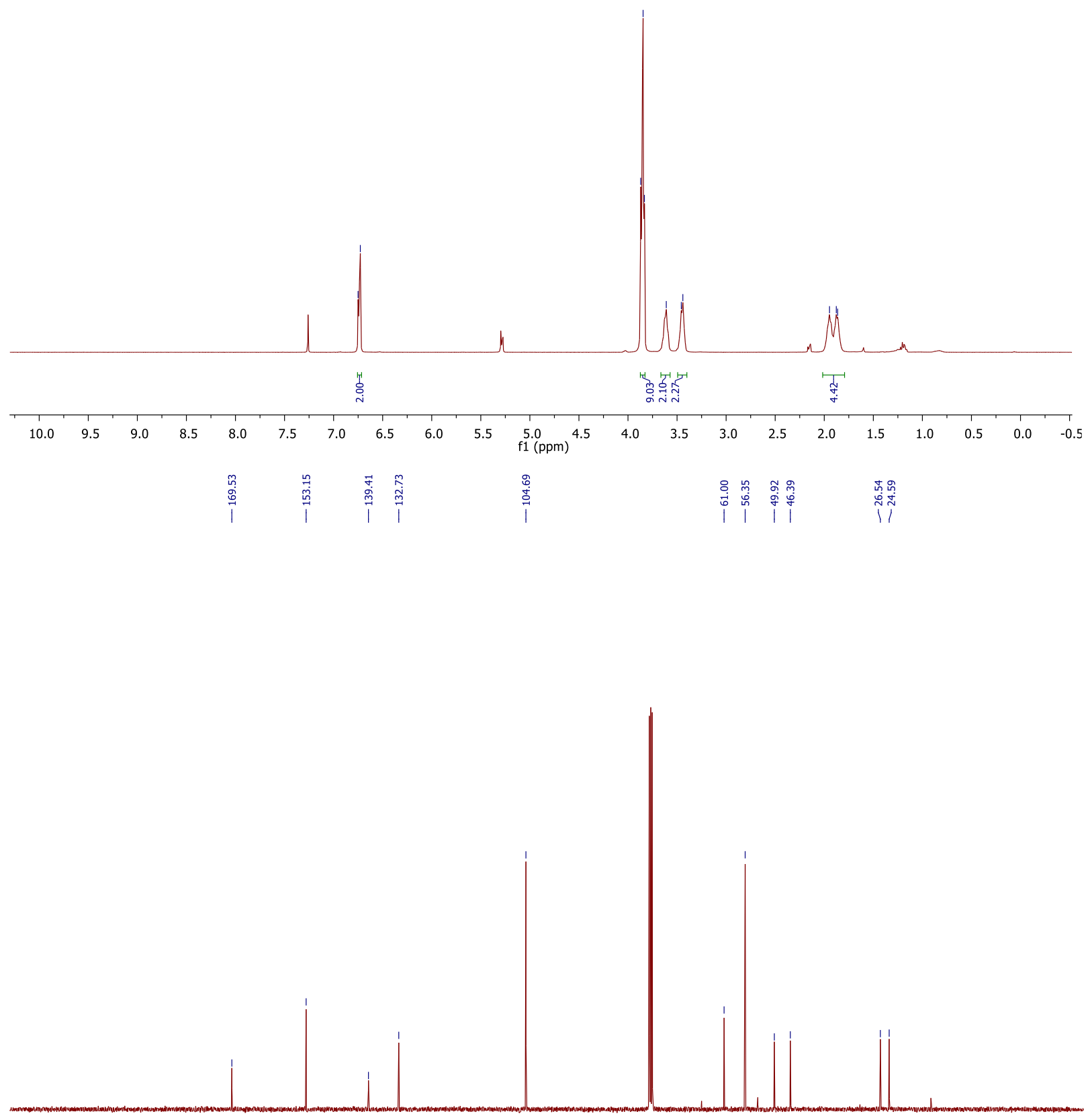

$\begin{array}{lllllllllllllllllllllll}210 & 200 & 190 & 180 & 170 & 160 & 150 & 140 & 130 & 120 & 110 & \underset{\mathrm{f} 1(\mathrm{ppm})}{100} & 90 & 80 & 70 & 60 & 50 & 40 & 30 & 20 & 10 & 0 & -10\end{array}$ 
(2-Bromo-5-methoxyphenyl)(pyrrolidin-1-yl)methanone (3m)

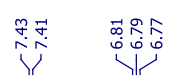

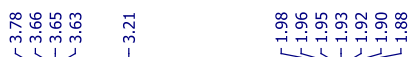<smiles>COc1ccc(Br)c(C(=O)N2CCCC2)c1</smiles>
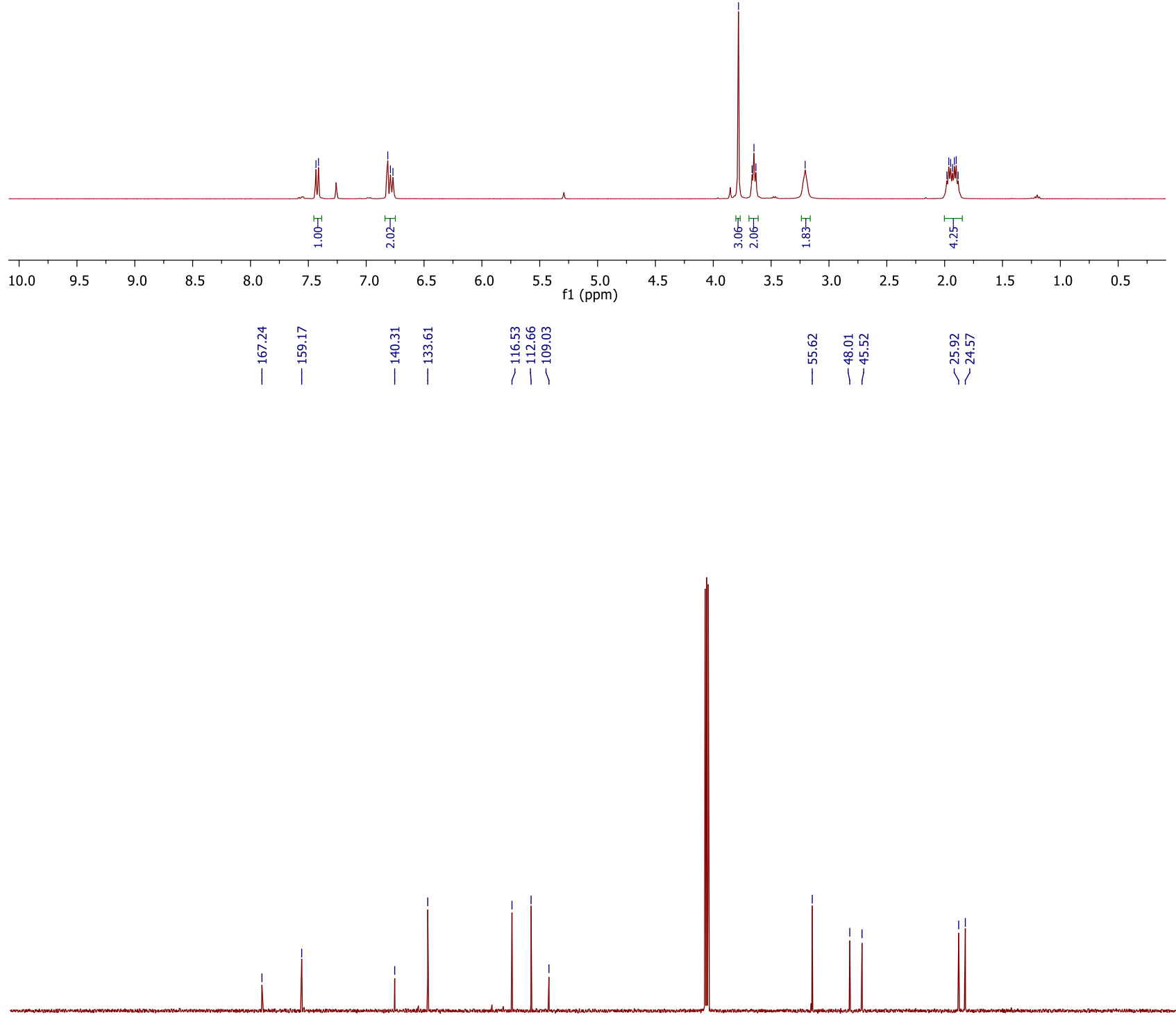

$\begin{array}{lllllllllllllllllllllll}210 & 200 & 190 & 180 & 170 & 160 & 150 & 140 & 130 & 120 & 110 & 100 & 90 & 80 & 70 & 60 & 50 & 40 & 30 & 20 & 10 & 0 & -10\end{array}$ 
(3-Chlorophenyl)(pyrrolidin-1-yl)methanone (3n)
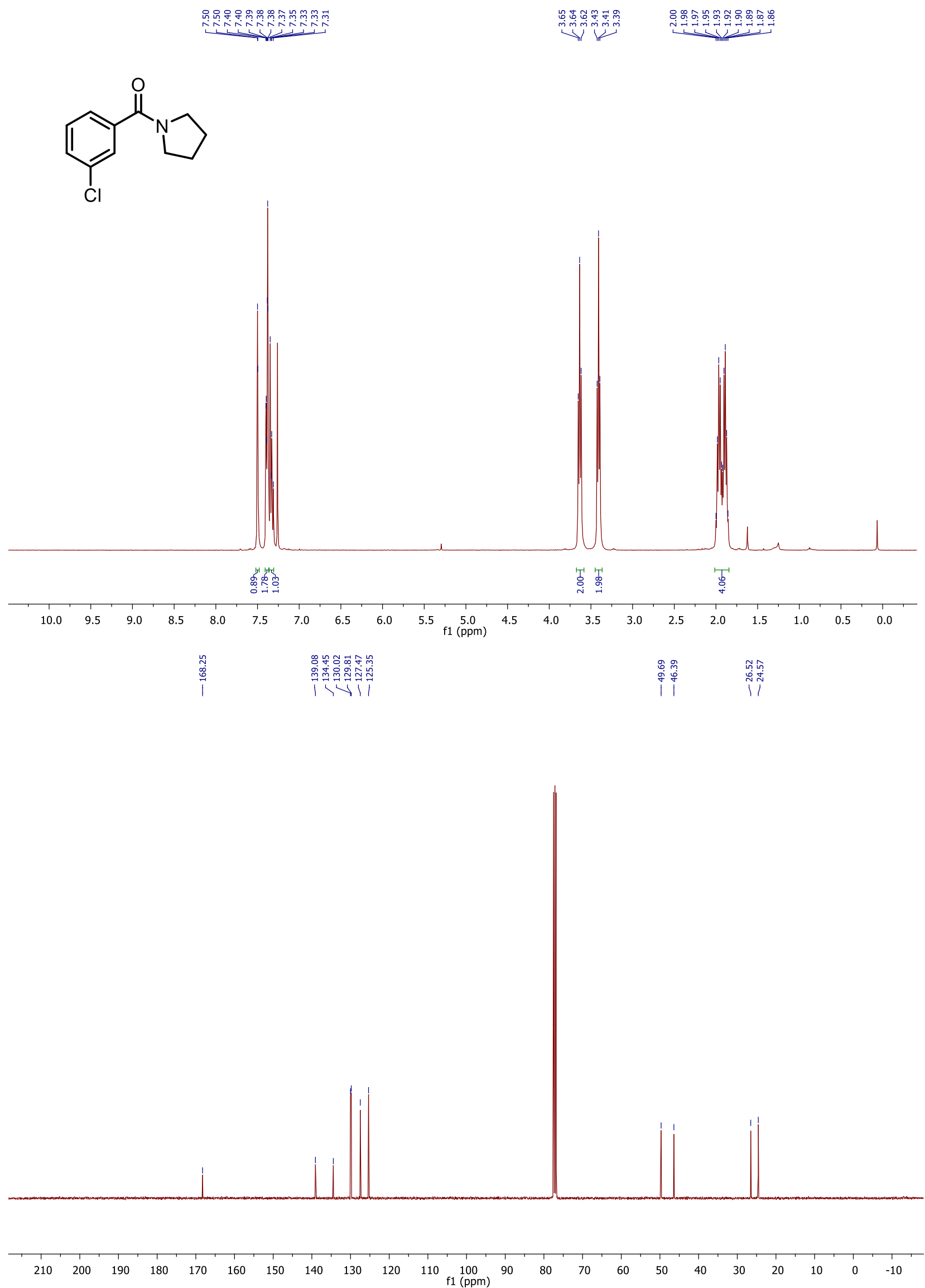
(2,3-Dihydro-1H-pyrrol-1-yl)(4-vinylphenyl)methanone (3p)
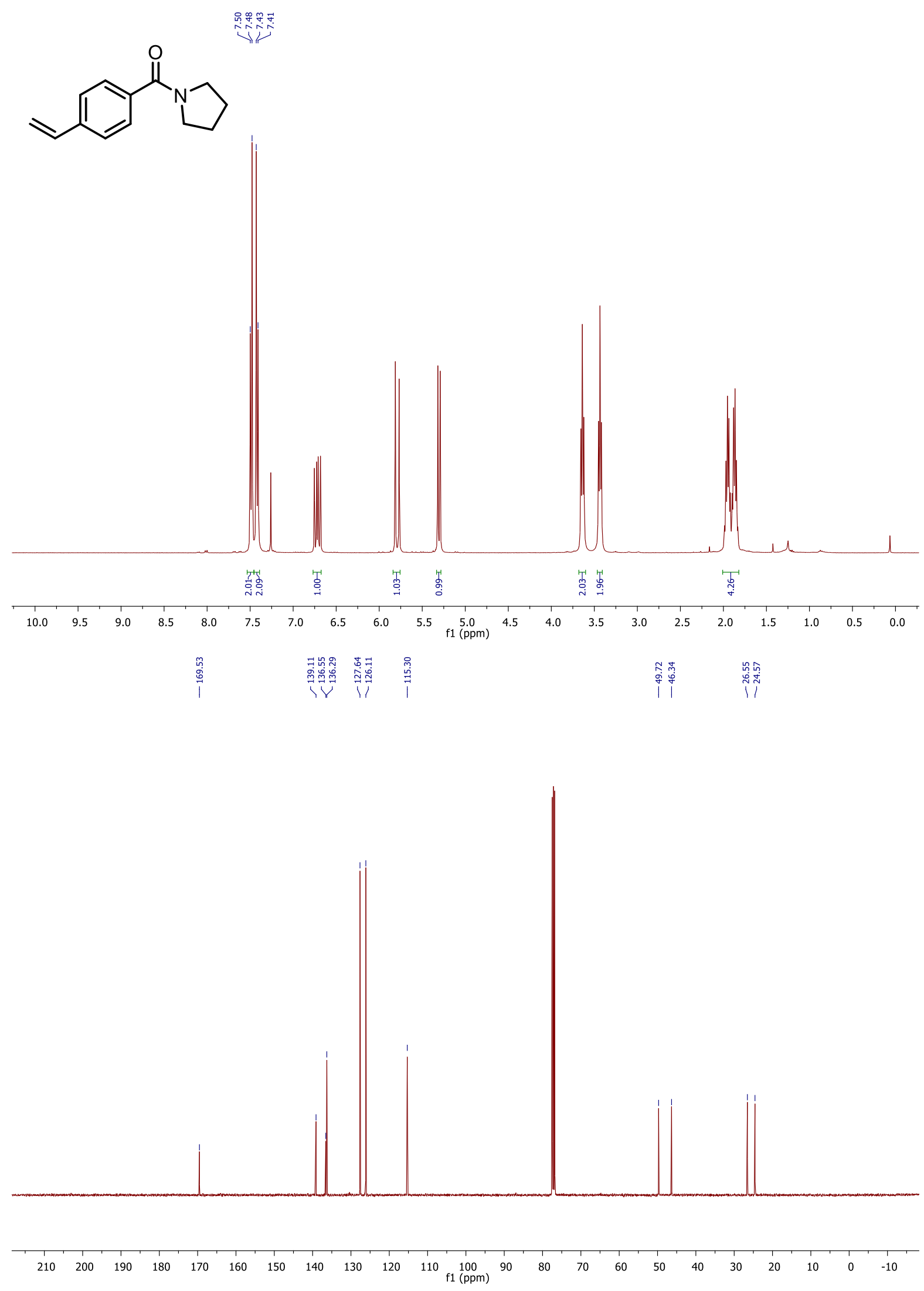

66 
(4-(Methylthio)phenyl)(piperidin-1-yl)methanone (3q)<smiles>C1=C2C[As]1[As]2</smiles><smiles>CSc1ccc(C(=O)N2CCCC2)cc1</smiles>
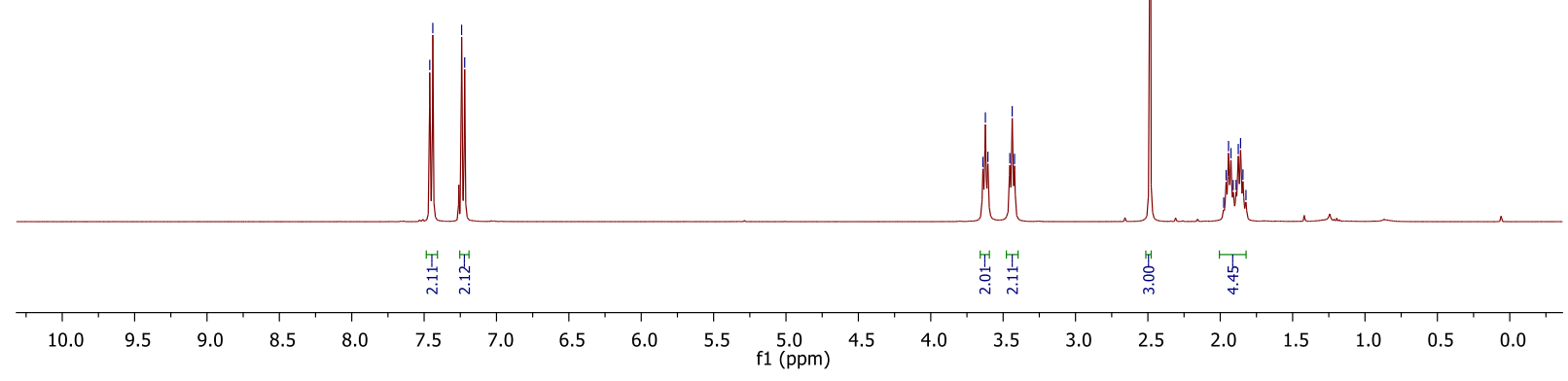
i

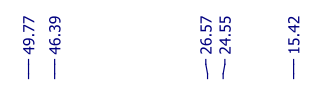

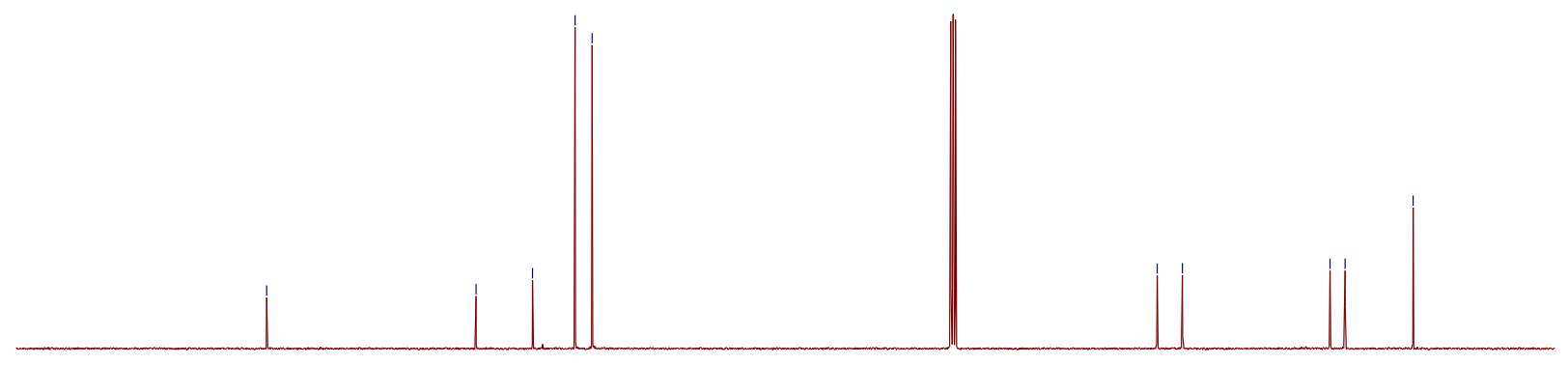

$200 \quad 190 \quad 180 \quad 170$

$\begin{array}{lllll}130 & 120 & 110 & \begin{array}{l}100 \\ \mathrm{f} 1(\mathrm{ppm})\end{array}\end{array}$

$90 \quad 80$

$70 \quad 60$

50

$\begin{array}{lllll}40 & 30 & 20 & 10 & 0\end{array}$ 
3-(Pyrrolidine-1-carbonyl)benzonitrile (3r)

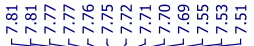

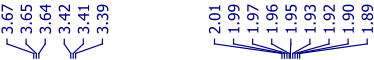<smiles>N#Cc1cccc(C(=O)N2CCCC2)c1</smiles>

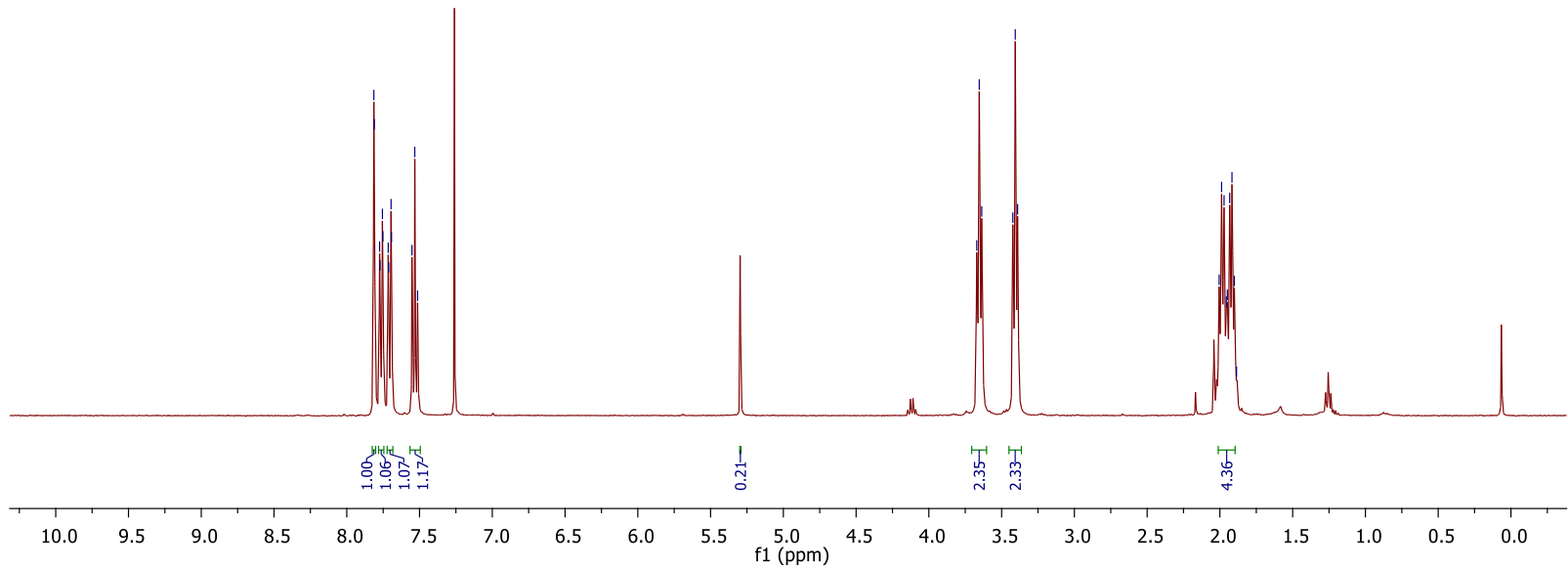

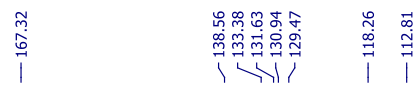

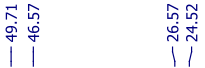

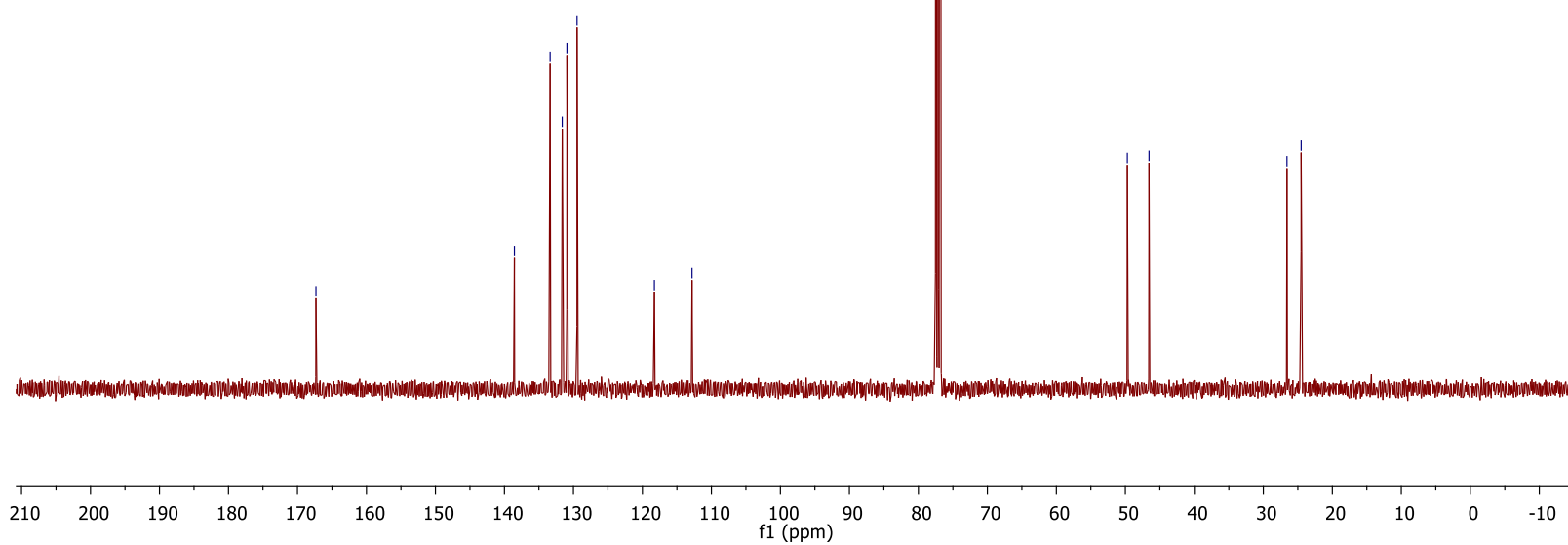


(4-Hydroxy-3-methoxyphenyl)(pyrrolidin-1-yl)methanone (precursor for 4v)

象

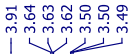

$\overbrace{\mathrm{OMe}}^{1}$

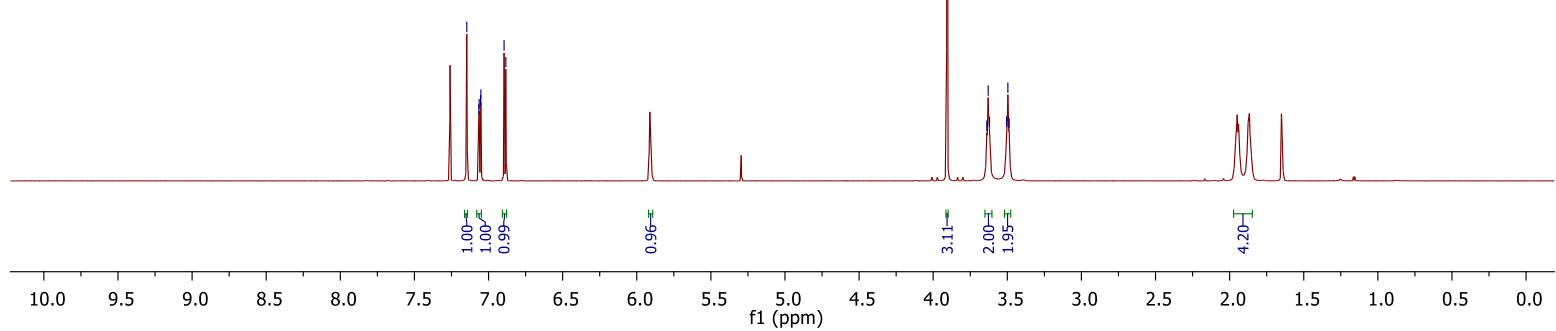

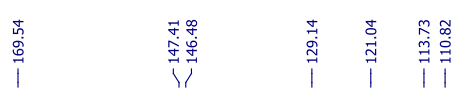

।

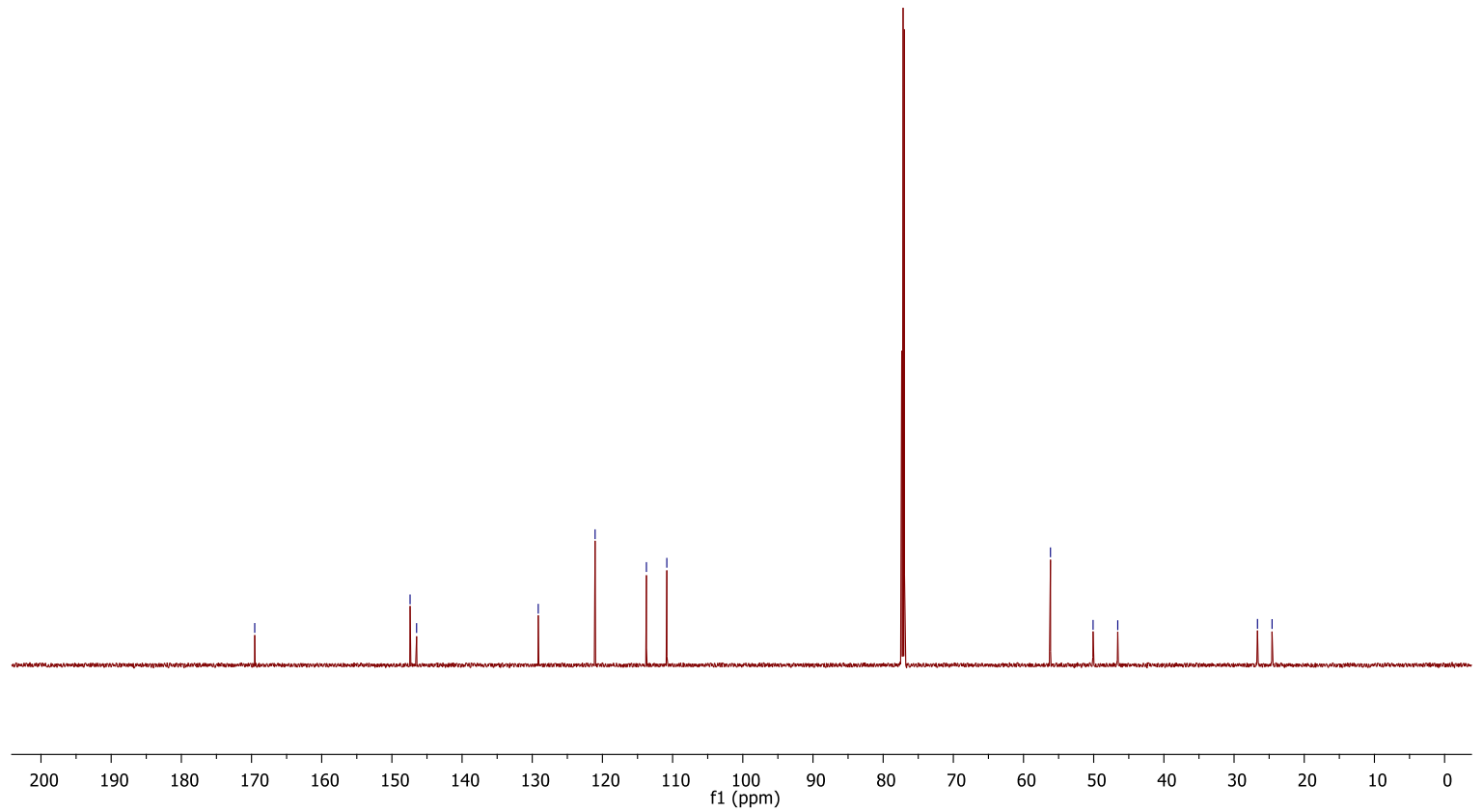



carboxylate (3v)
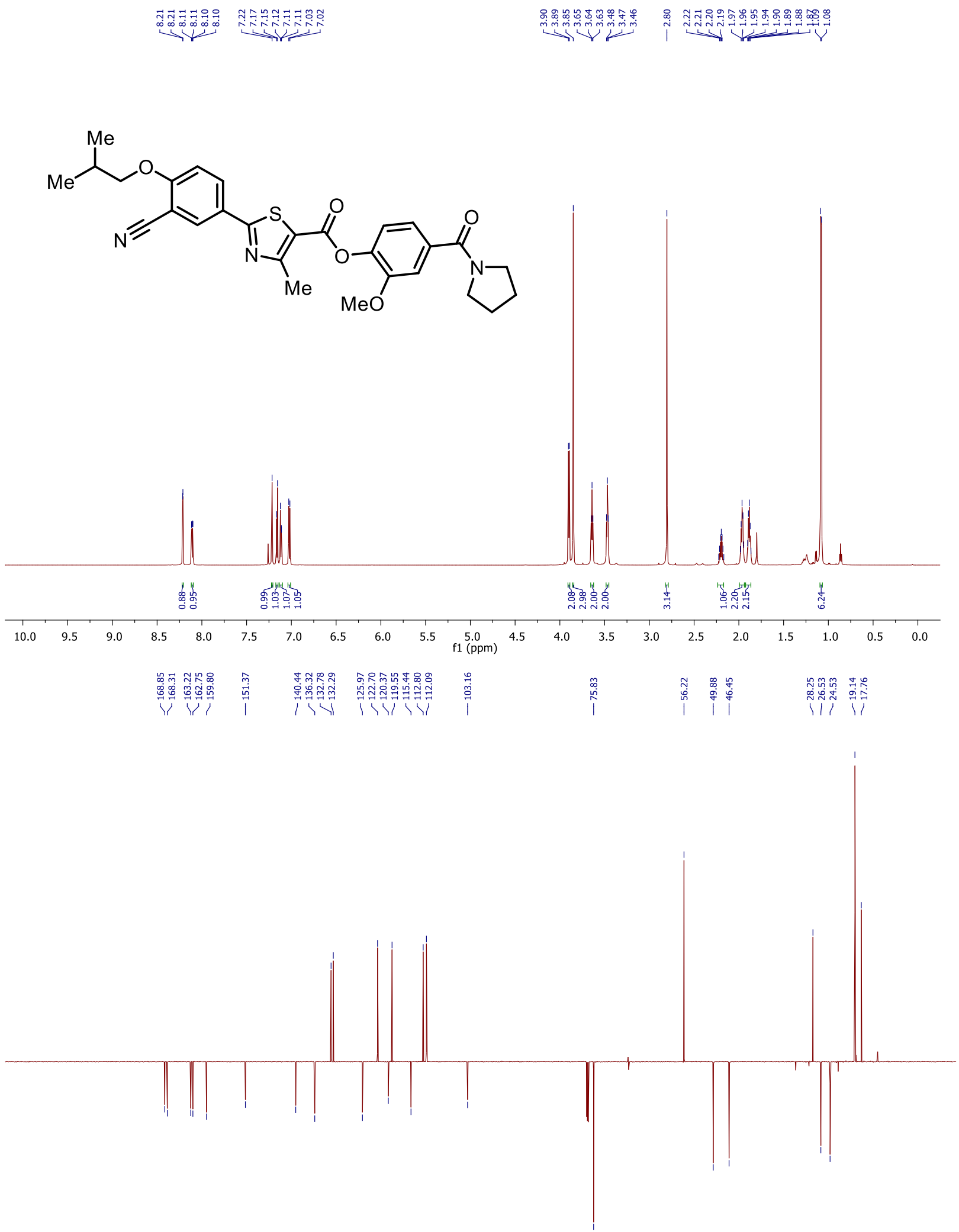


\section{(3,4-Dihydropyridin-1(2H)-yl)(mesityl)methanone (2a)}

资<smiles>Cc1cc(C)c(C(=O)N2C=CCCC2)c(C)c1</smiles>
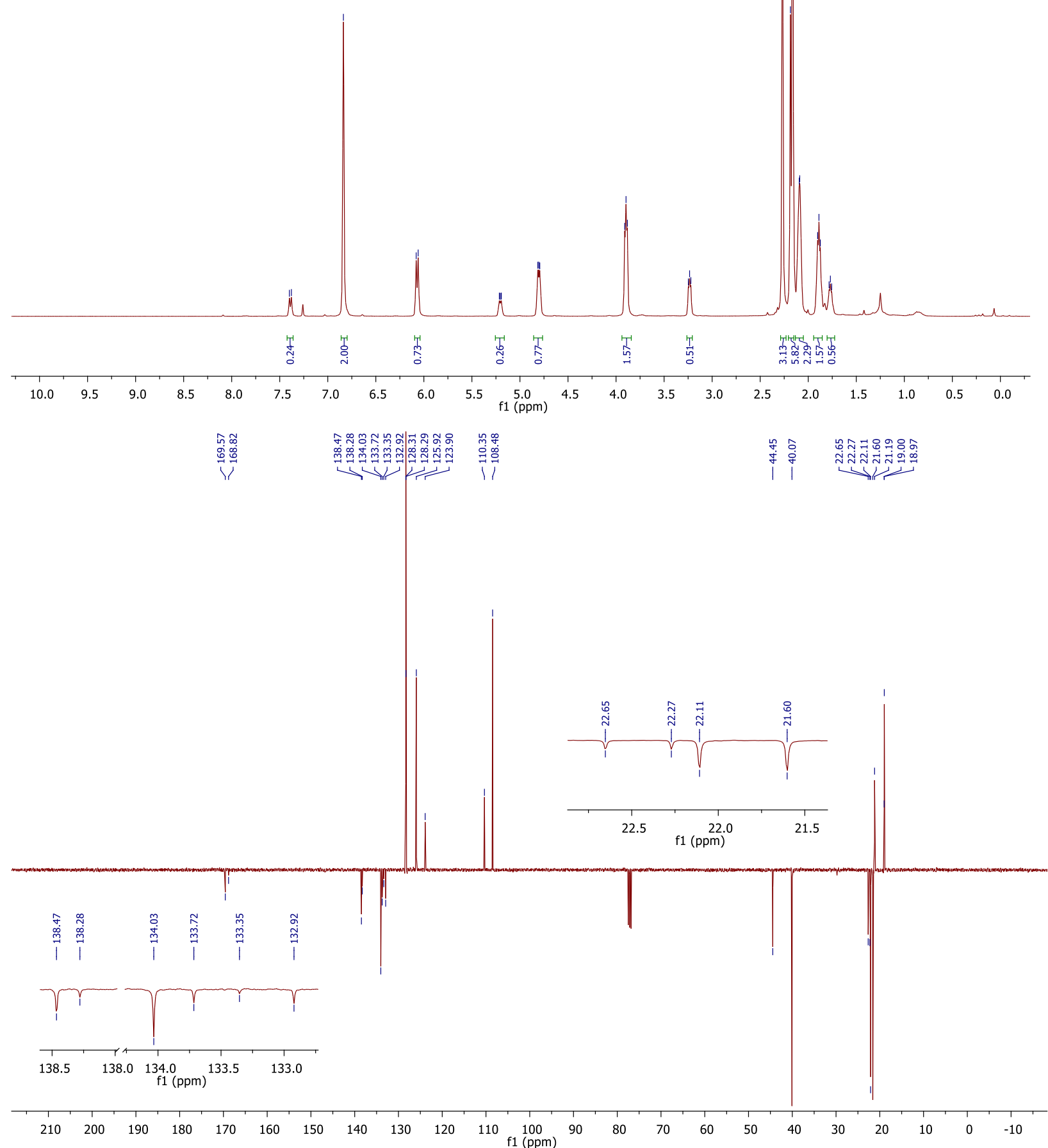
(2,3-Dihydro-1H-pyrrol-1-yl)(mesityl)methanone (2b)

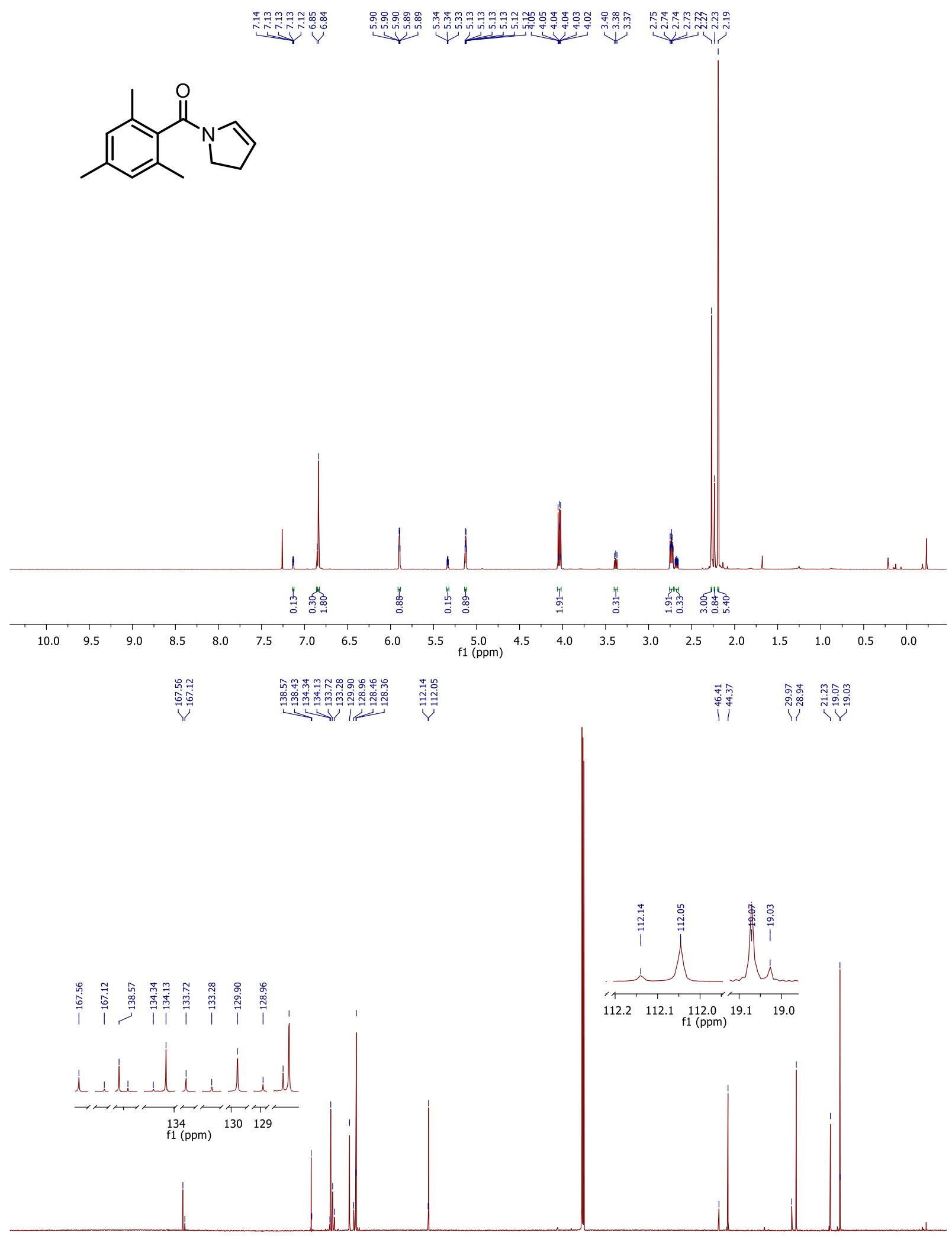

$\begin{array}{lllllllllllllllllllllllll}200 & 190 & 180 & 170 & 160 & 150 & 140 & 130 & 120 & 110 & 100 & 90 & 80 & 70 & 60 & 50 & 40 & 30 & 20 & 10 & 0\end{array}$ 
Mesityl(2,3,4,5-tetrahydro-1H-azepin-1-yl)methanone (2c)

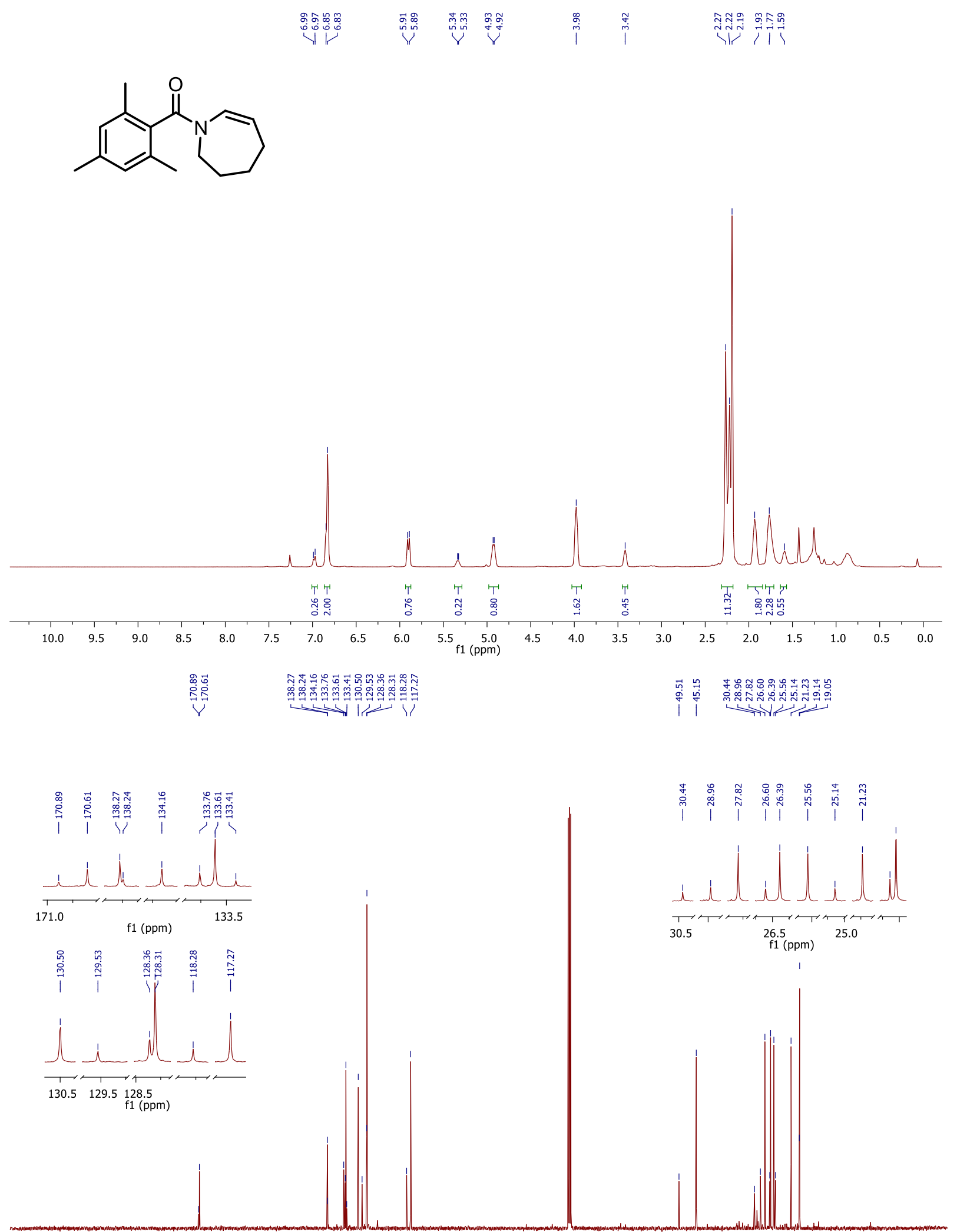

$\begin{array}{lllllllllllllllllllllllllllll}210 & 200 & 190 & 180 & 170 & 160 & 150 & 140 & 130 & 120 & 110 & 100 & 90 & 80 & 70 & 60 & 50 & 40 & 30 & 20 & 10 & 0 & -10\end{array}$ 
Mesityl(1,4-dioxa-8-azaspiro[4.5]dec-6-en-8-yl)methanone (2d)

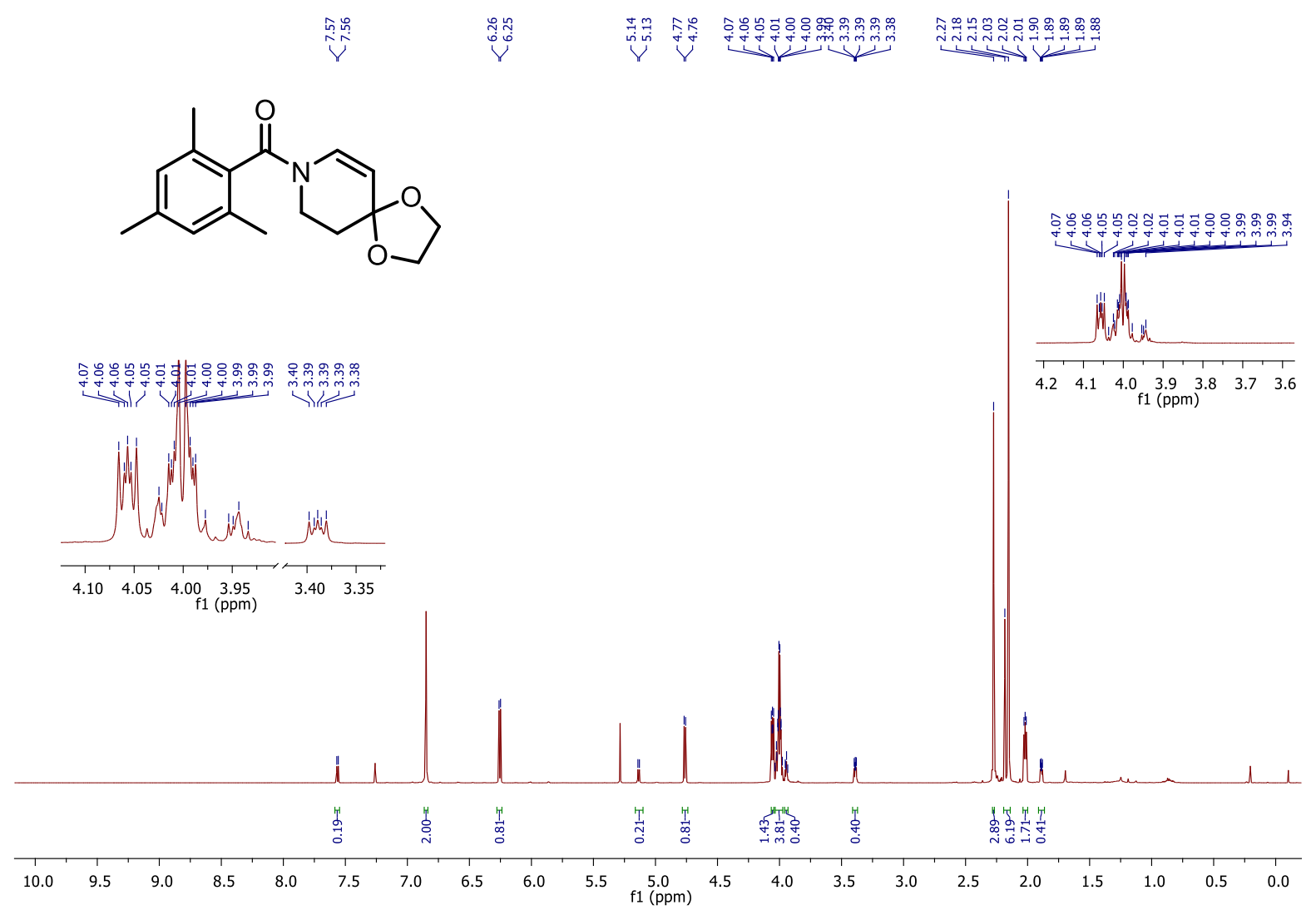

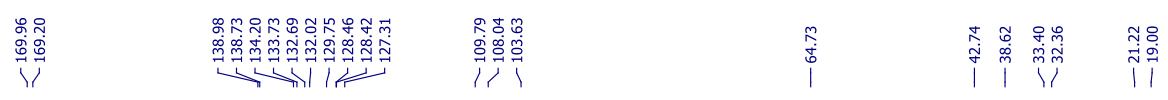

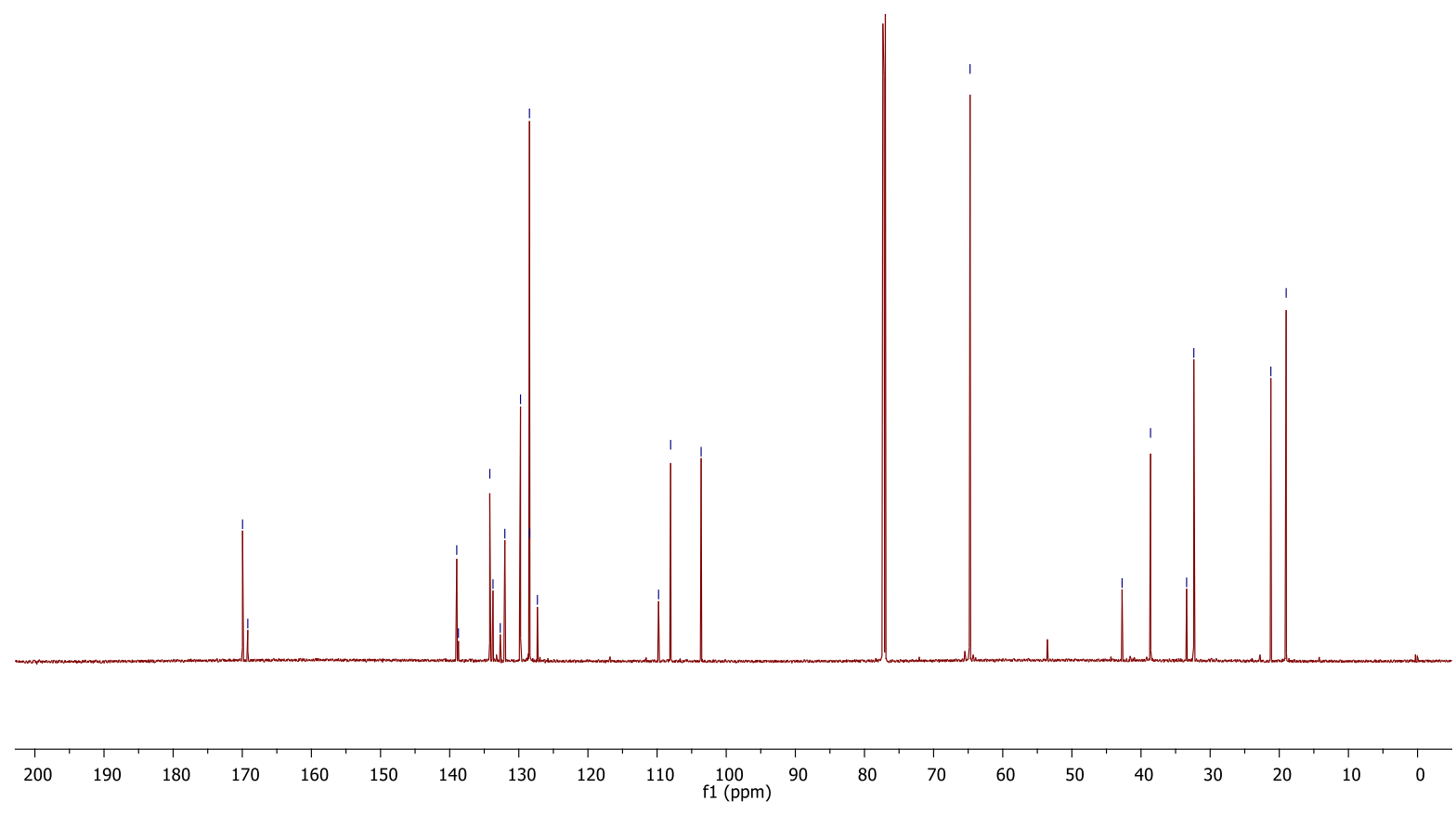




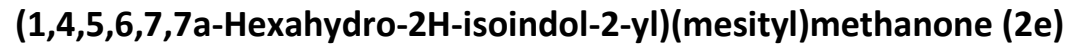
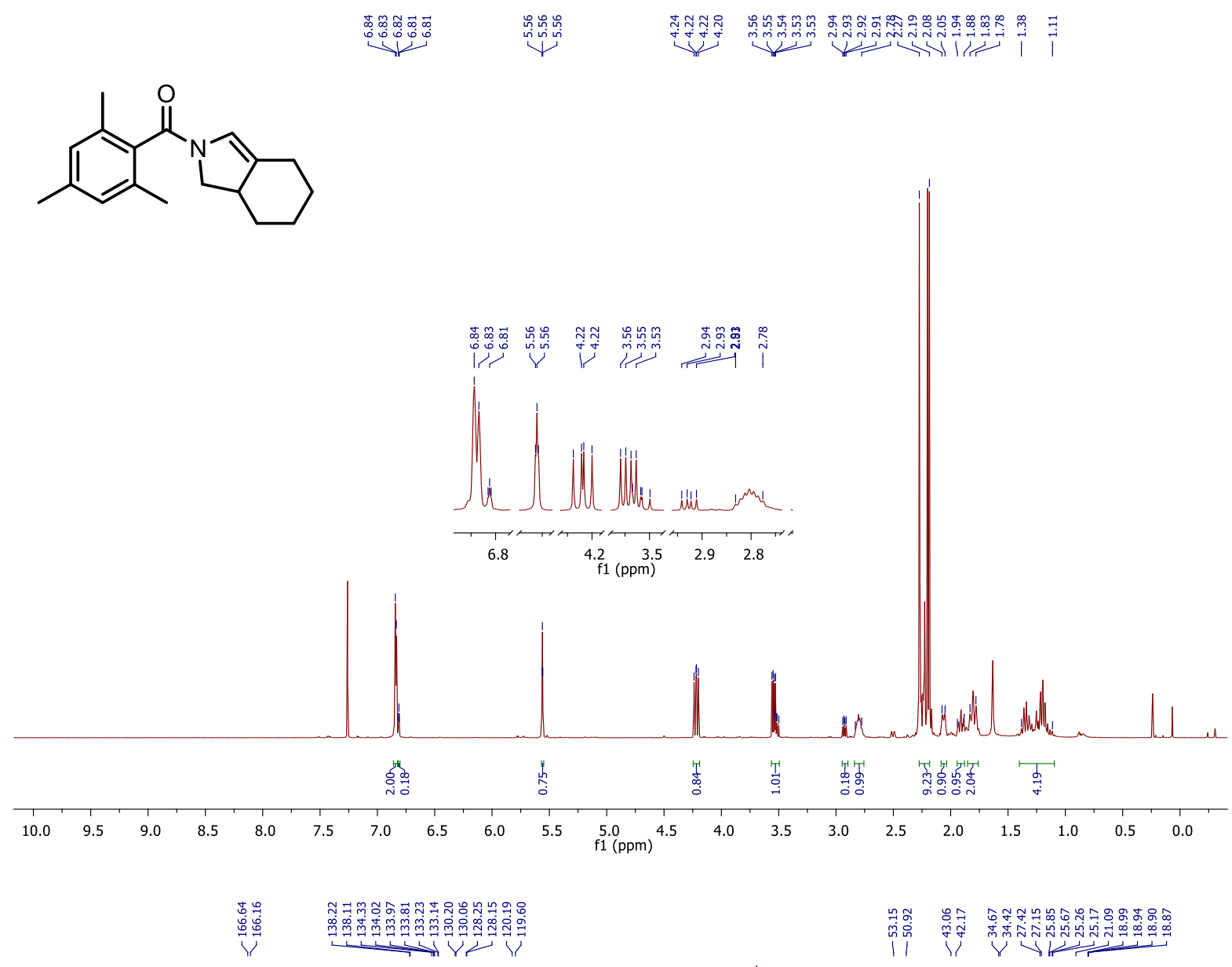

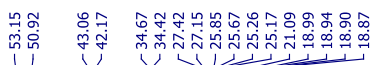

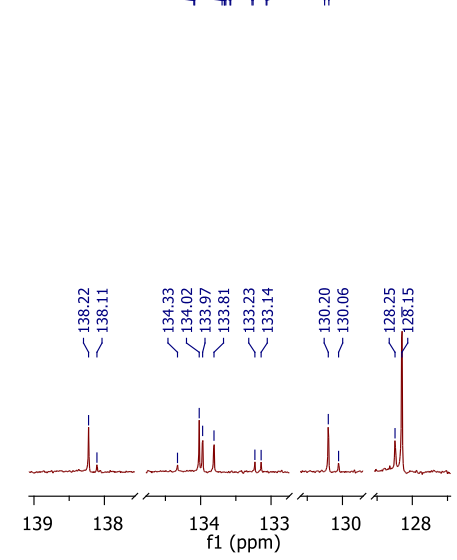

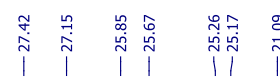

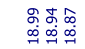
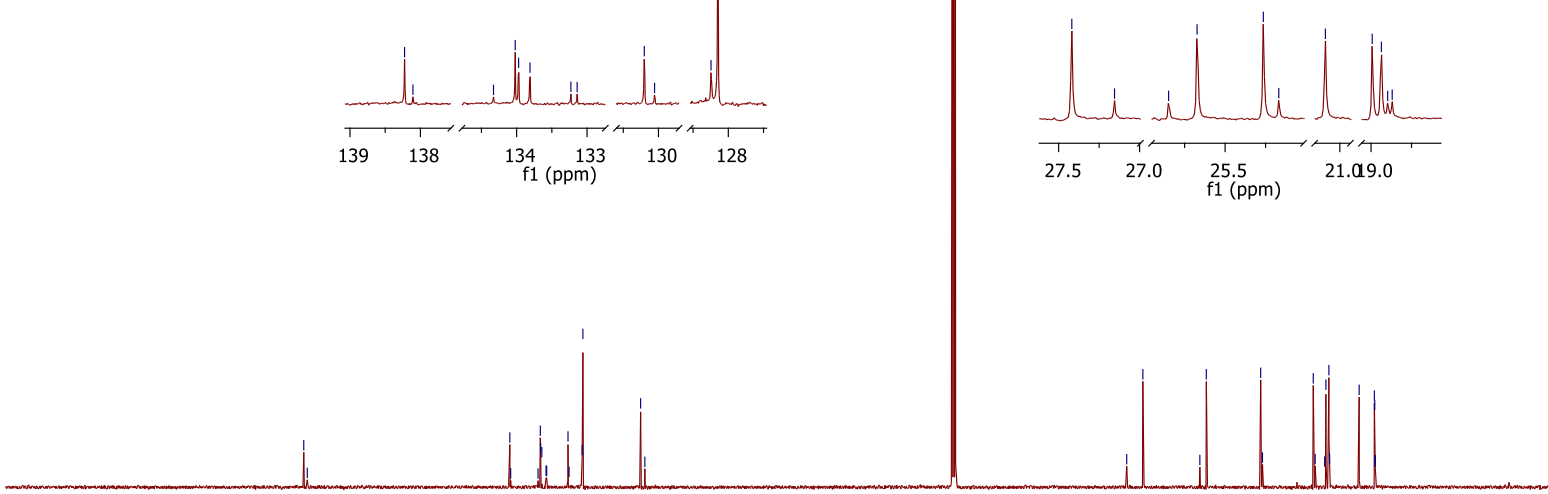

$\begin{array}{lllllllllll}200 & 190 & 180 & 170 & 160 & 150 & 140 & 130 & 120 & 110 & 100\end{array}$ 
(2,3-Dihydro-4H-1,4-oxazin-4-yl)(mesityl)methanone (2f)

丩丶<smiles>Cc1cc(C)c(C(=O)N2C=COCC2)c(C)c1</smiles>

thr

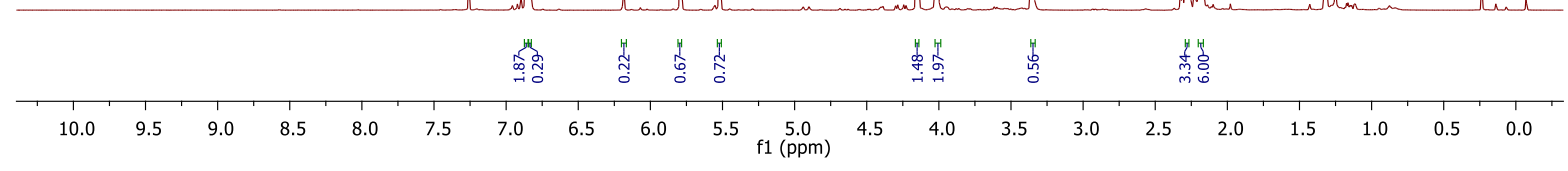

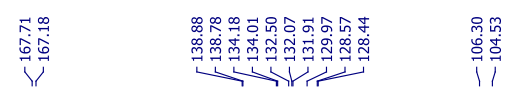

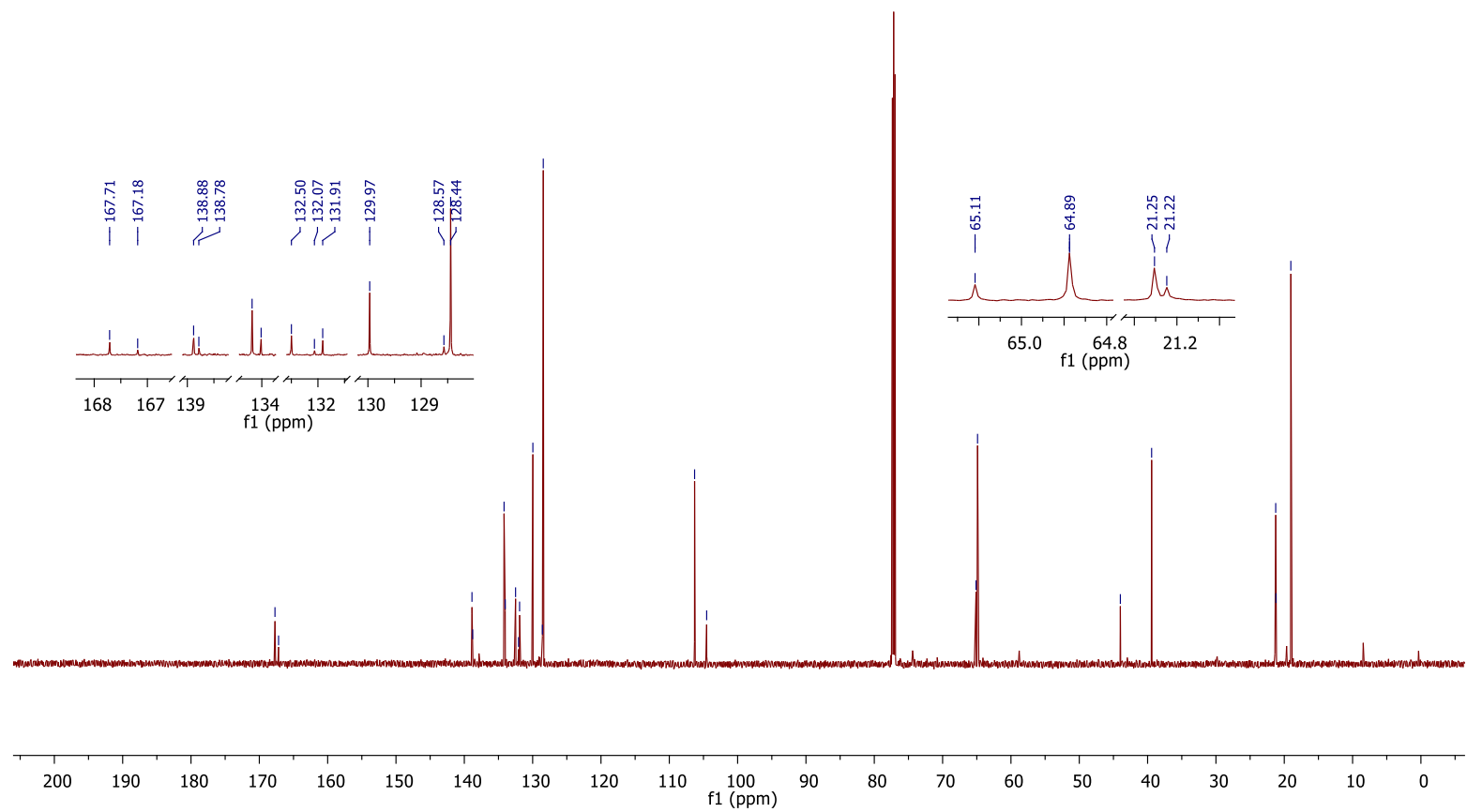


Ethyl 4-(2,4,6-trimethylbenzoyl)-3,4-dihydropyrazine-1(2H)-carboxylate (2g)

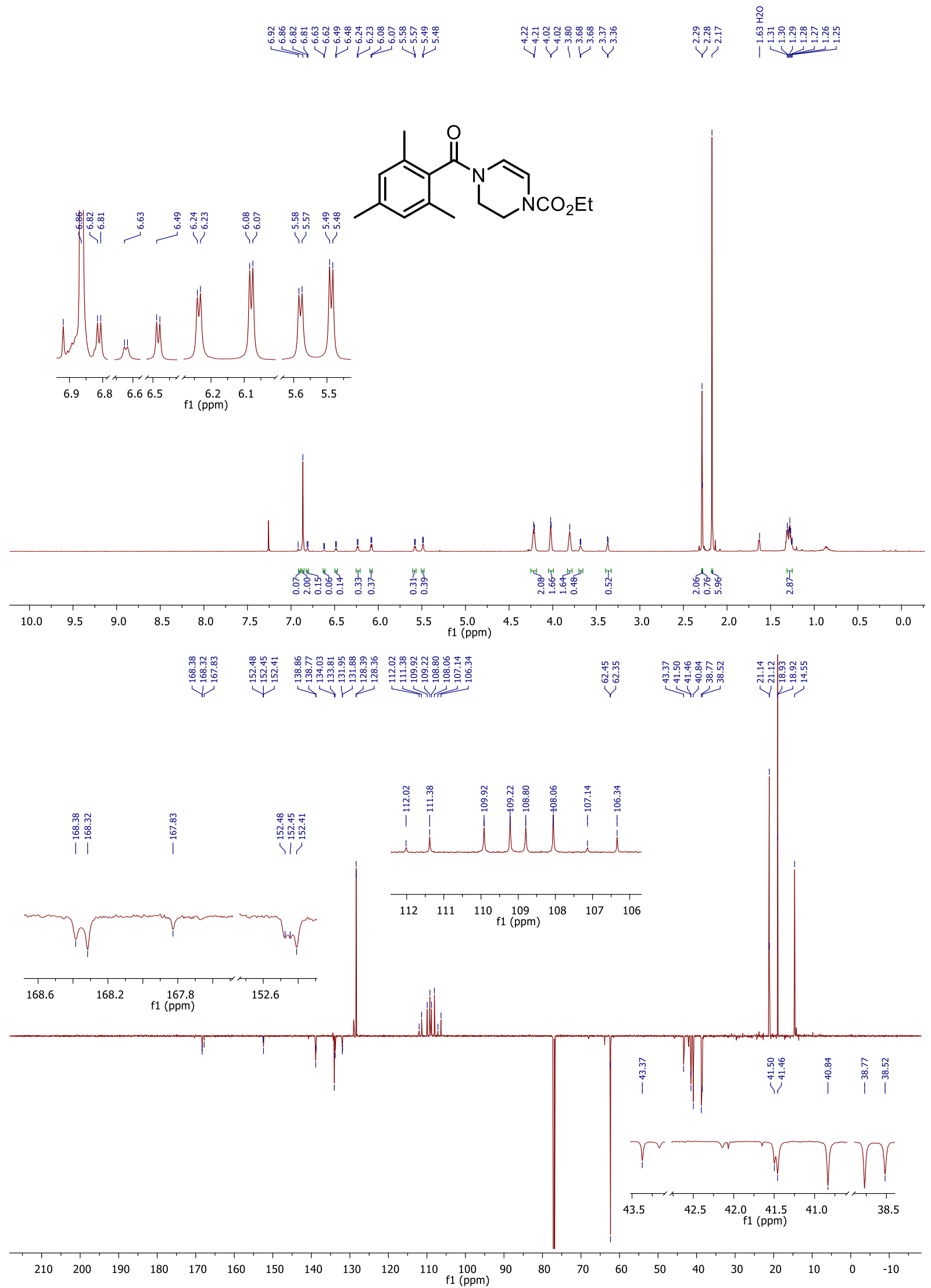


$\mathrm{N}$-Ethyl-2,4,6-trimethyl-N-vinylbenzamide (2h)
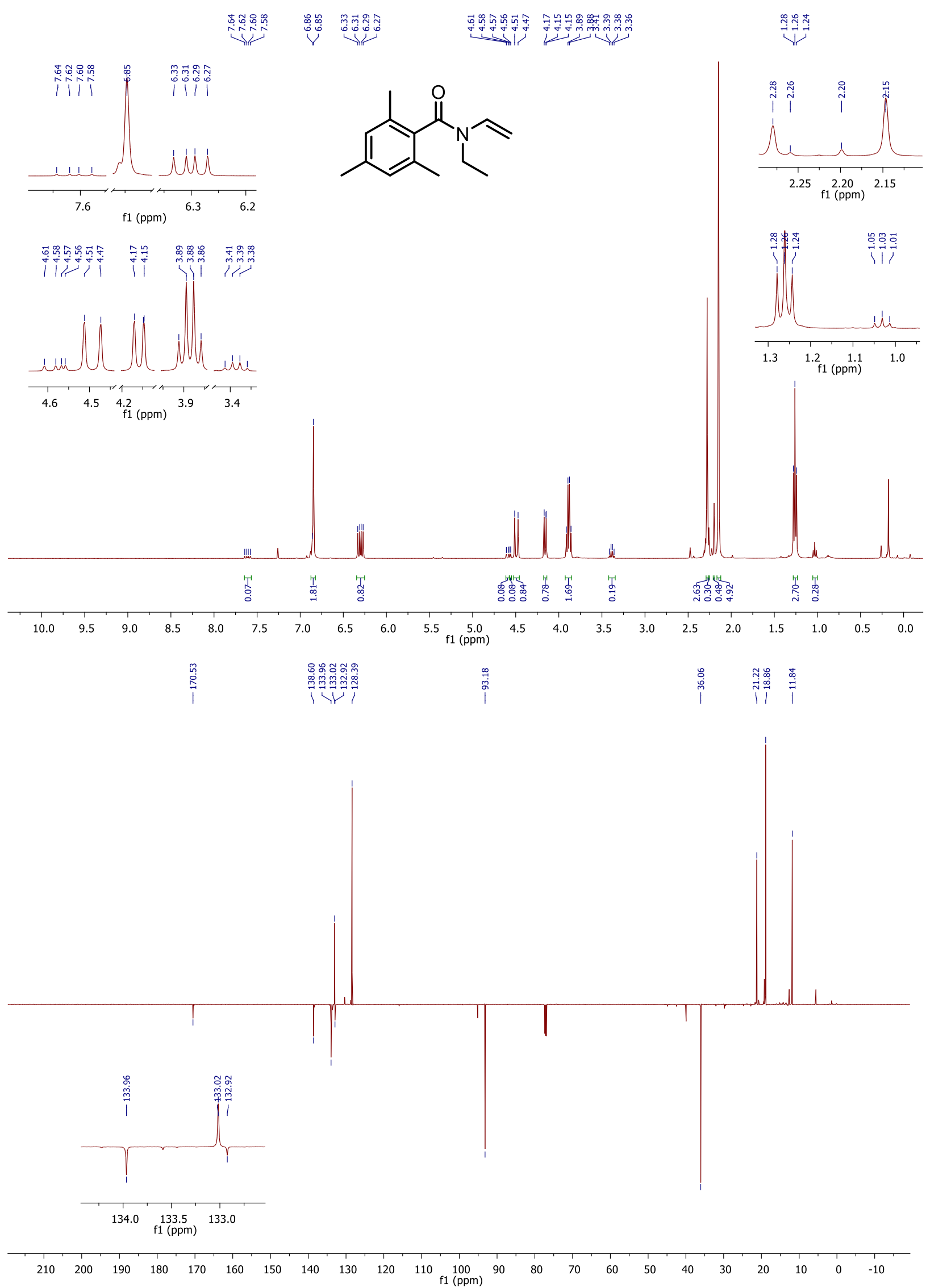

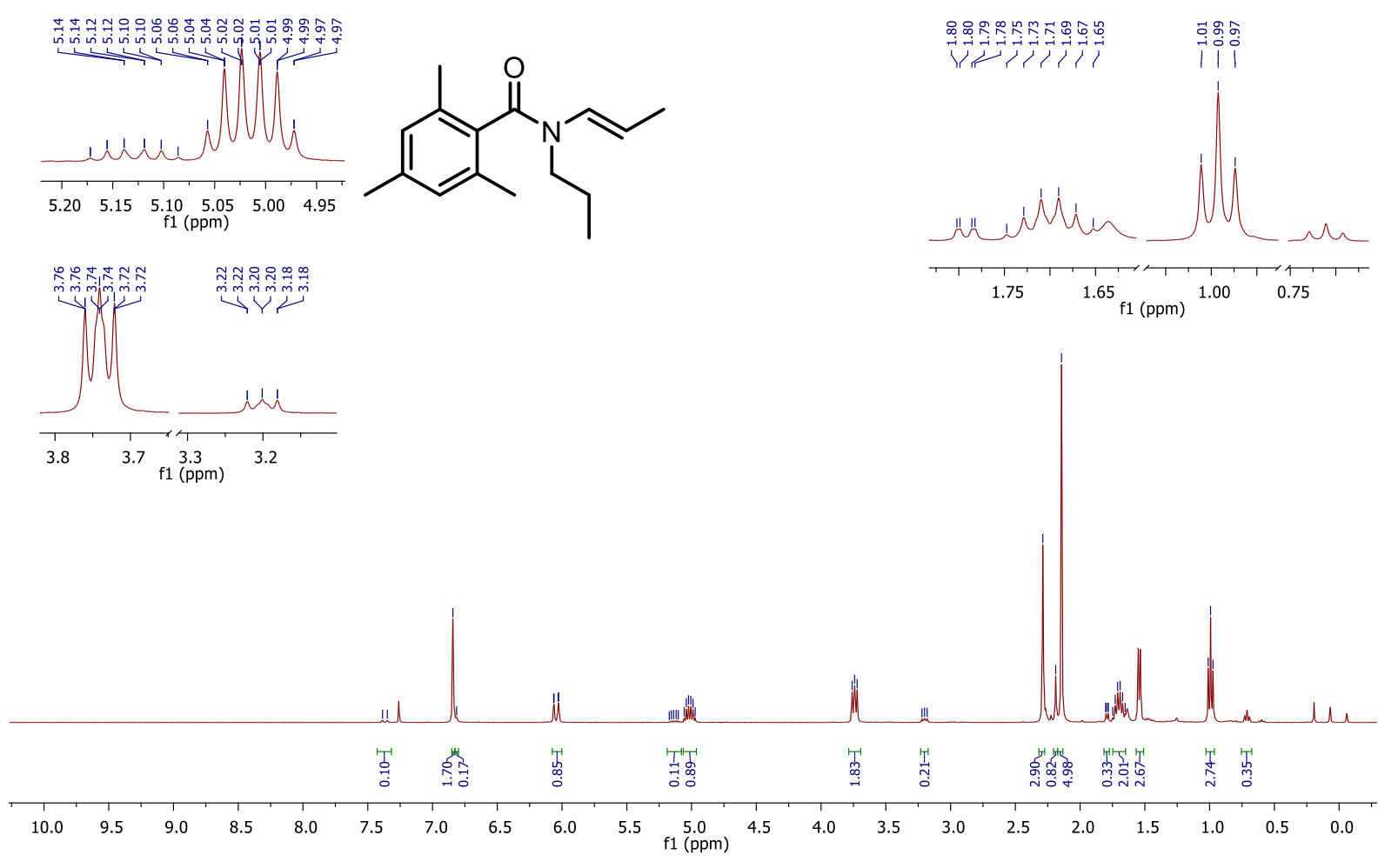
Vo

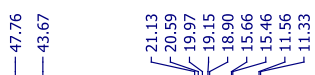

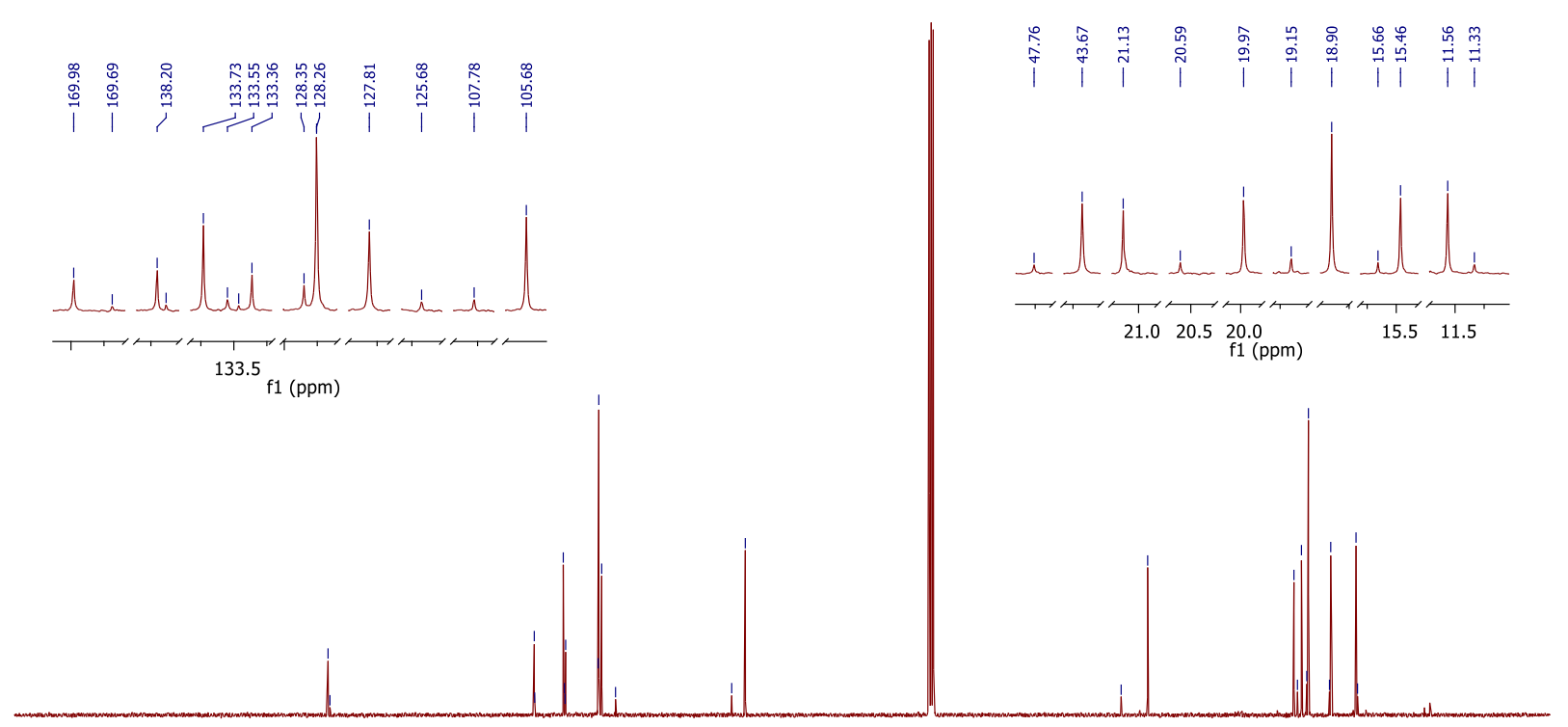

$\begin{array}{llllllllllllllllllllllllllll}210 & 200 & 190 & 180 & 170 & 160 & 150 & 140 & 130 & 120 & 110 & 100 & 90 & 80 & 70 & 60 & 50 & 40 & 30 & 20 & 10 & 0 & -10\end{array}$ 
(E)-N-(But-1-en-1-yl)-N-butyl-2,4,6-trimethylbenzamide (2j)
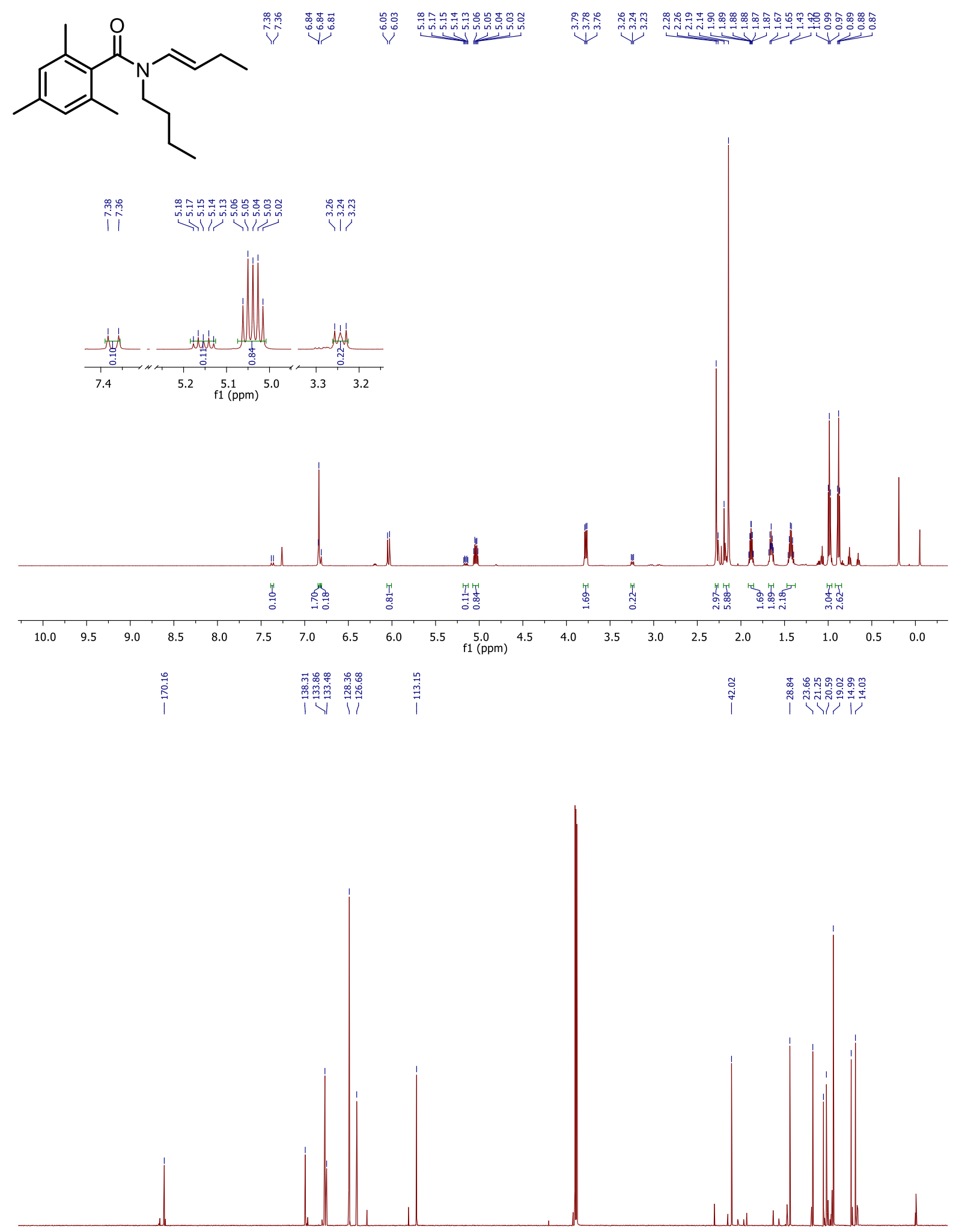

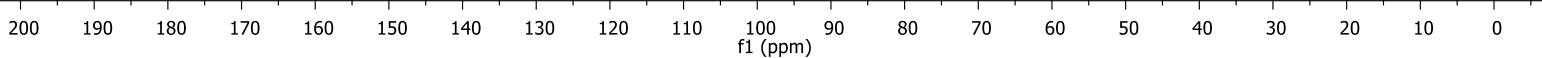




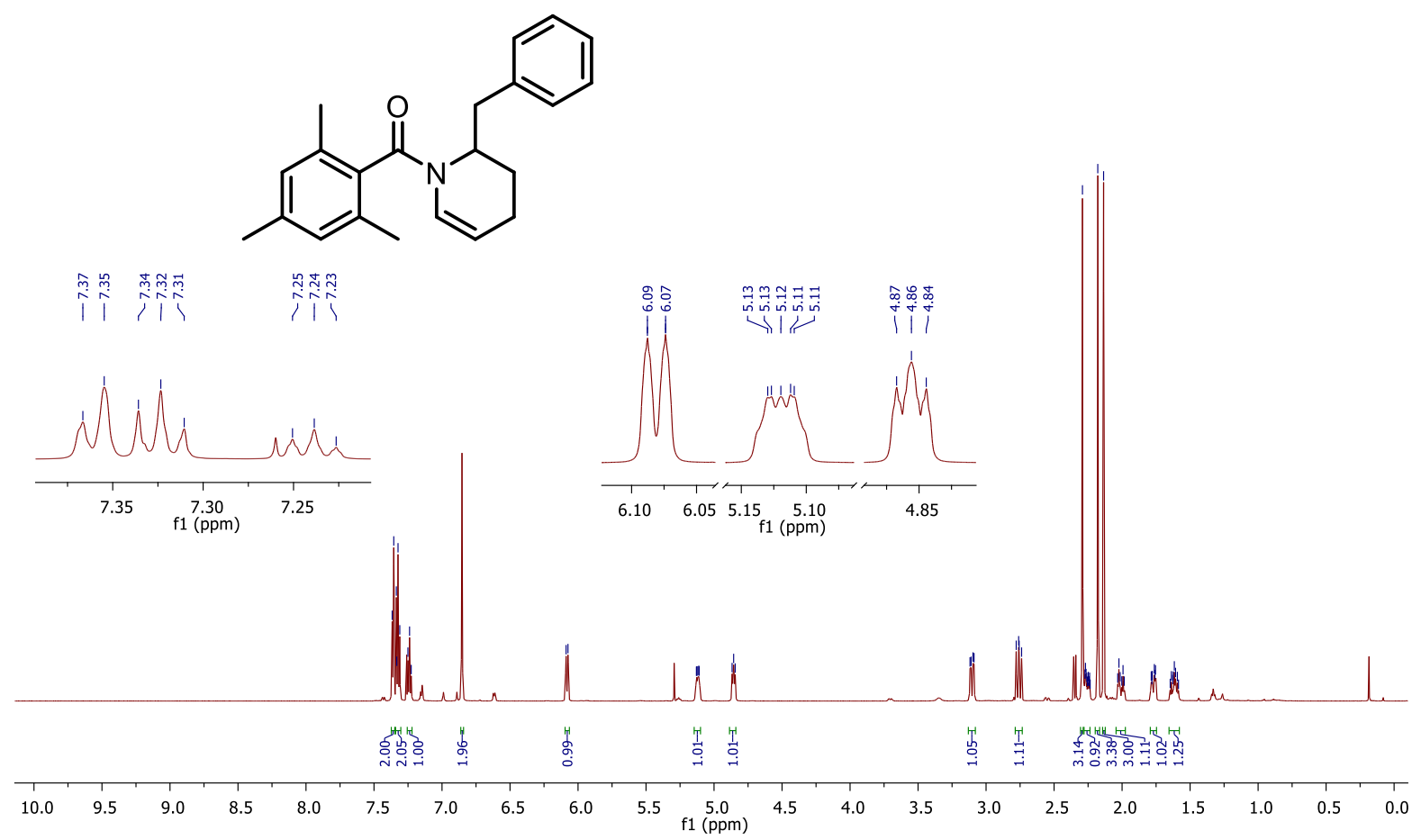

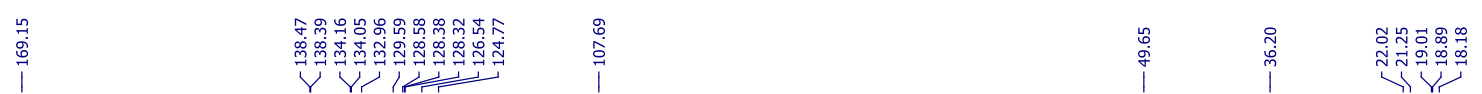

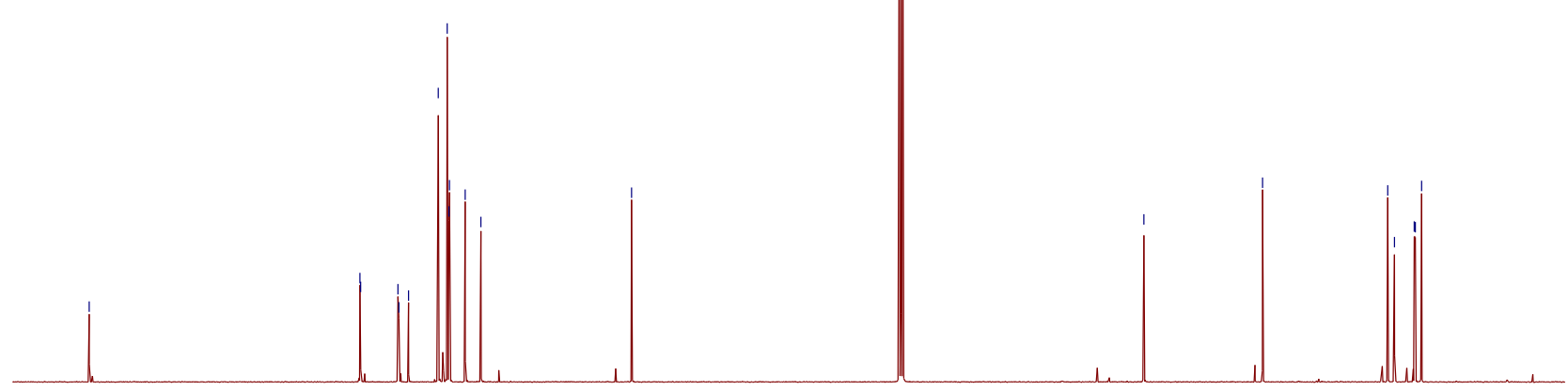

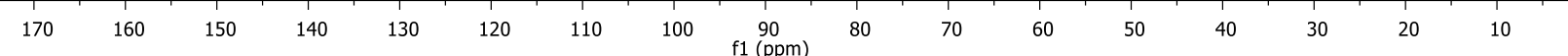


Mesityl(6-methyl-3,4-dihydropyridin-1(2H)-yl)methanone (2I)
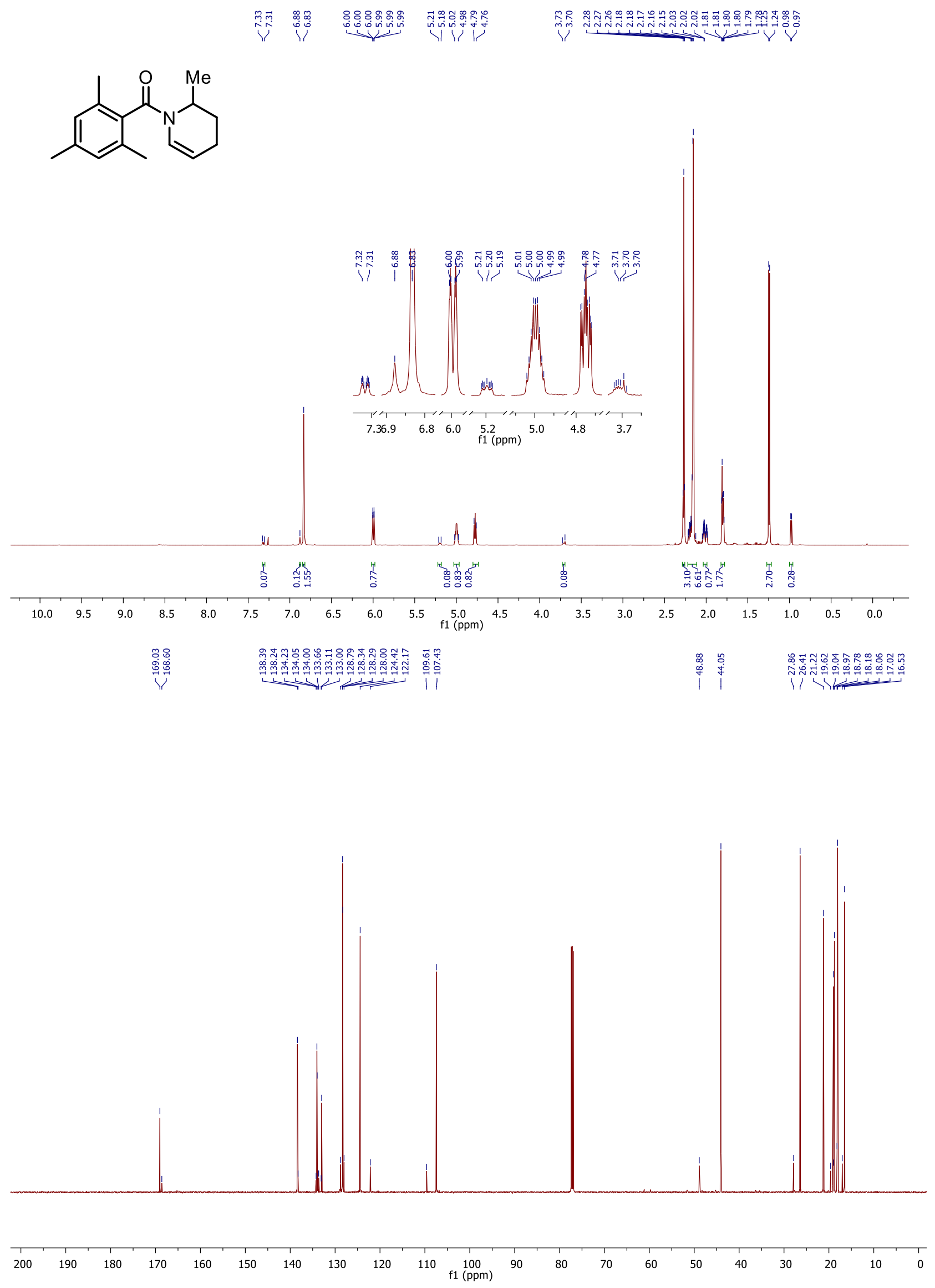
Mesityl(2-phenyl-2,3-dihydro-1H-pyrrol-1-yl)methanone (2m)

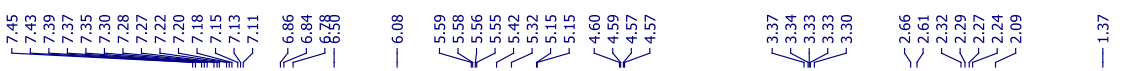<smiles>Cc1cc(C)c(C(=O)N2C=CCC2c2ccccc2)c(C)c1</smiles>

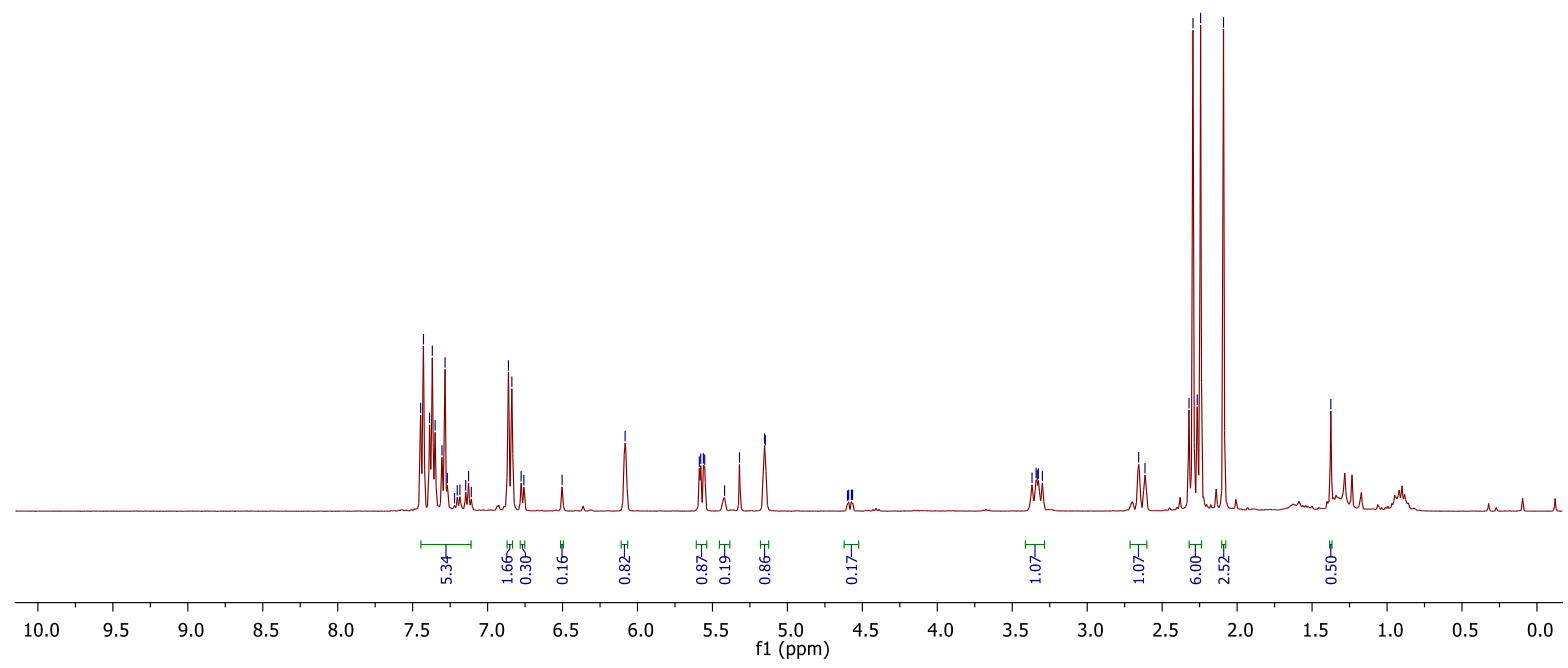

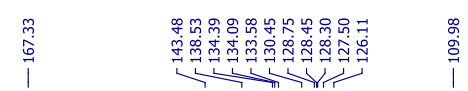

$\underset{\substack{\infty \\ 0}}{\substack{0 \\ 0}}$

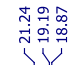

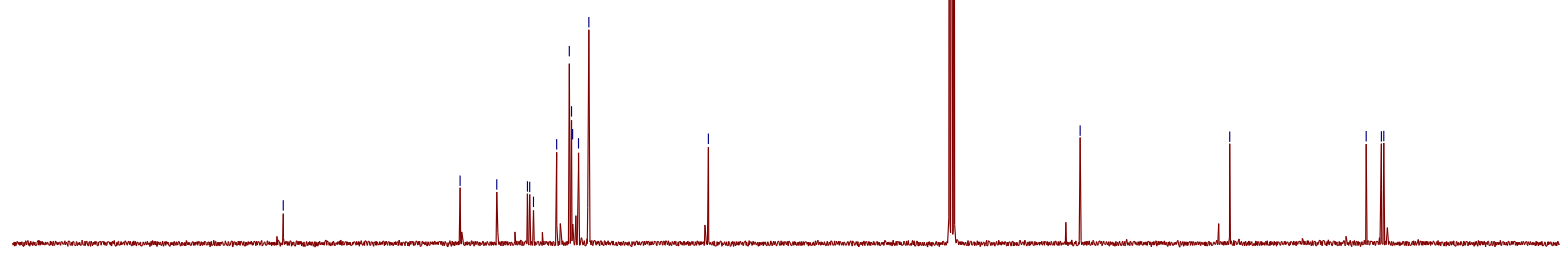

$\begin{array}{lllllllllllllllllllllll}200 & 190 & 180 & 170 & 160 & 150 & 140 & 130 & 120 & 110 & \begin{array}{c}100 \\ \mathrm{f} 1(\mathrm{ppm})\end{array} & 90 & 80 & 70 & 60 & 50 & 40 & 30 & 20 & 10 & 0\end{array}$ 
<smiles>Cc1cc(C)c(C(=O)N2C=CC[C@H]3CCCC[C@H]32)c(C)c1</smiles>

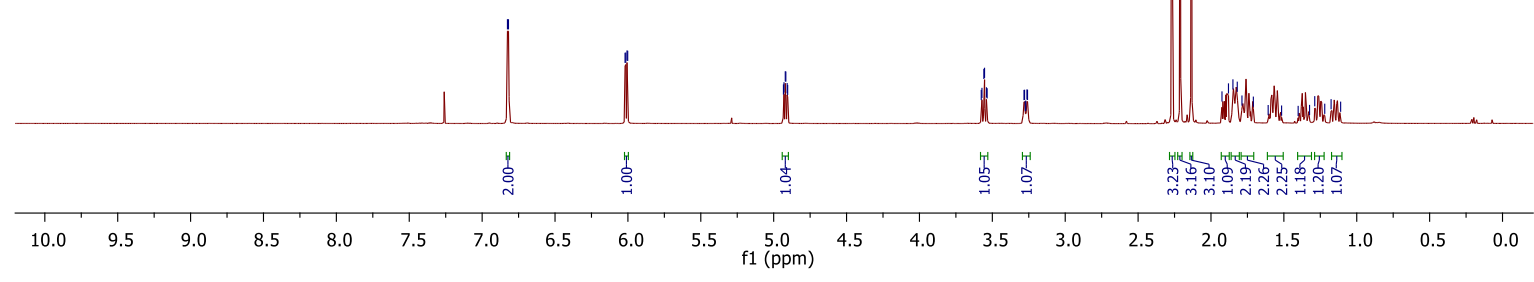

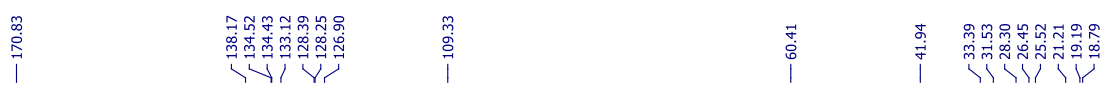

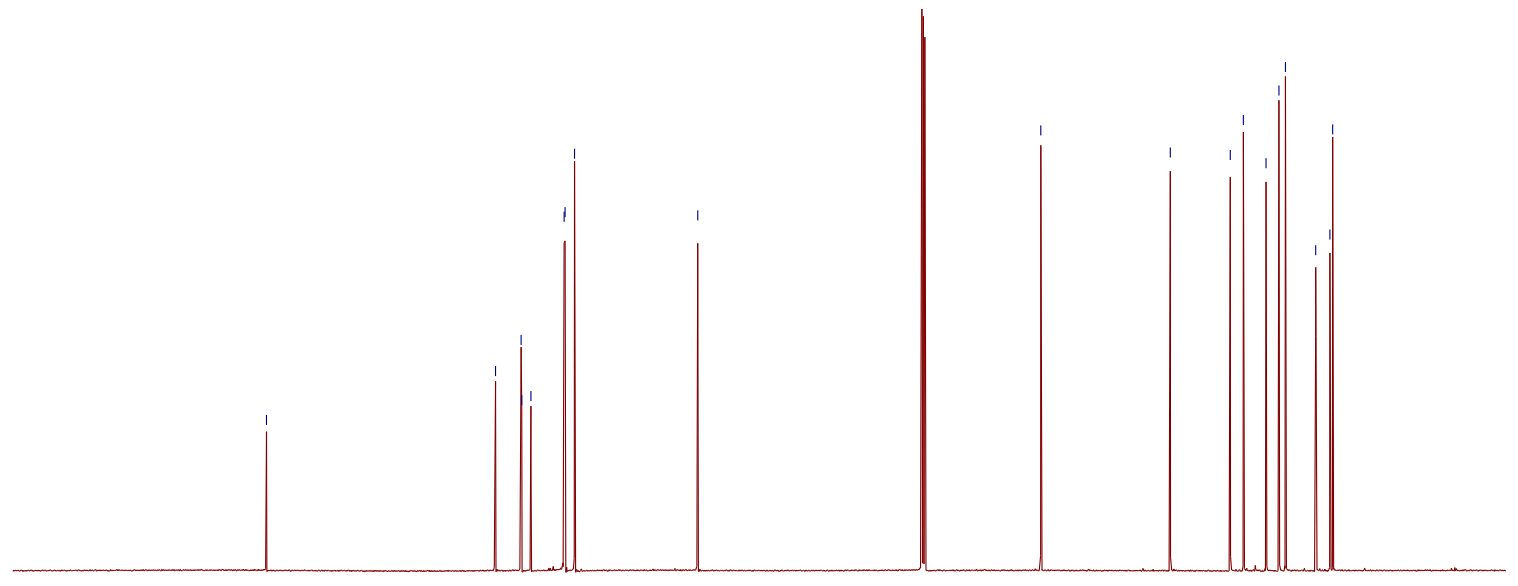

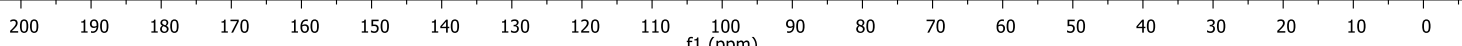


$\mathrm{N}$-Cyclohexyl-2,4,6-trimethyl-N-vinylbenzamide (20)
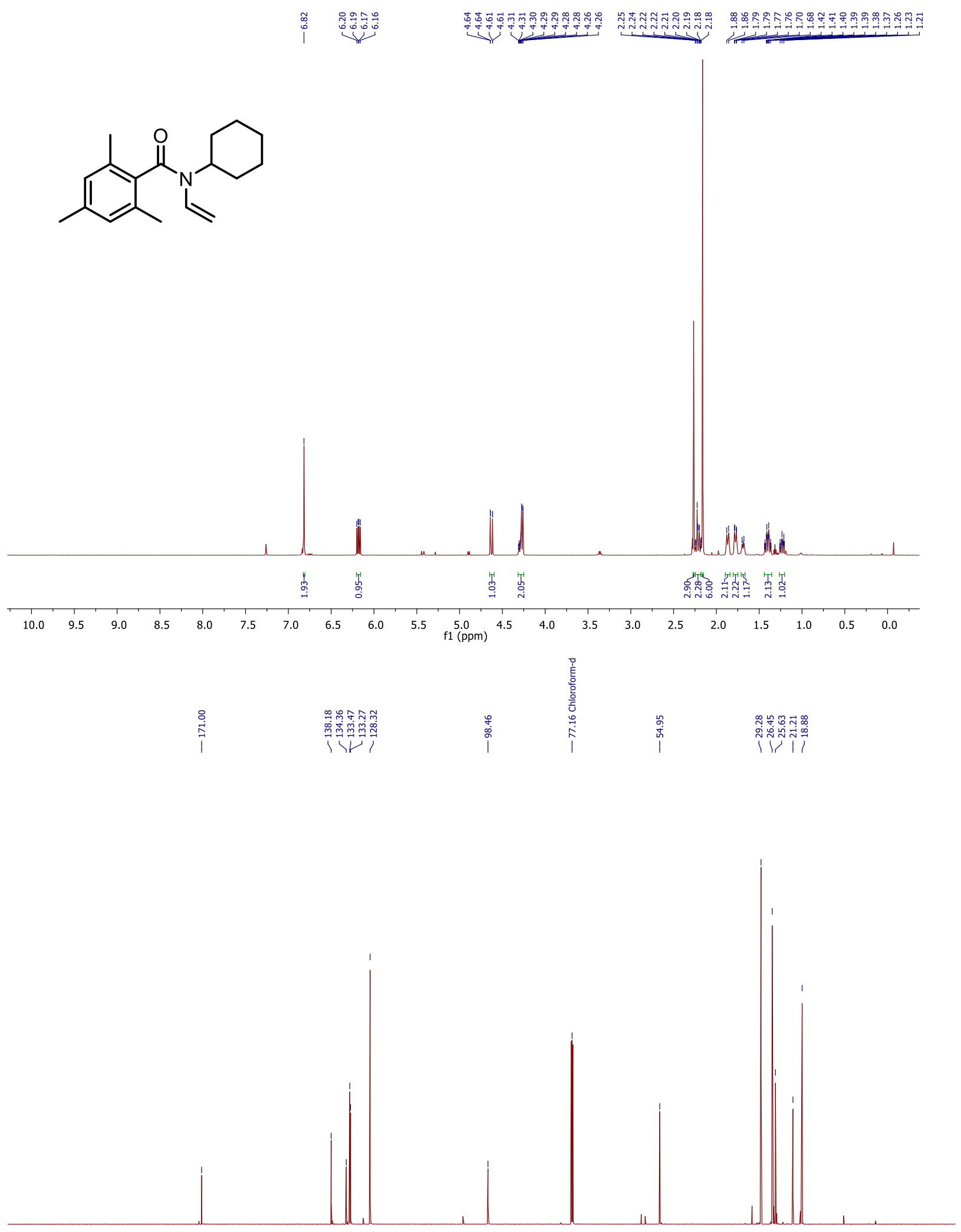

$\begin{array}{lllllllllllllllllllllllllll}220 & 210 & 200 & 190 & 180 & 170 & 160 & 150 & 140 & 130 & 120 & 110 & \begin{array}{c}100 \\ 100\end{array} & 90 & 80 & 70 & 60 & 50 & 40 & 30 & 20 & 10 & 0 & -10\end{array}$ 
$\mathrm{N}$-Butyl-2,4,6-trimethyl-N-vinylbenzamide (2p)

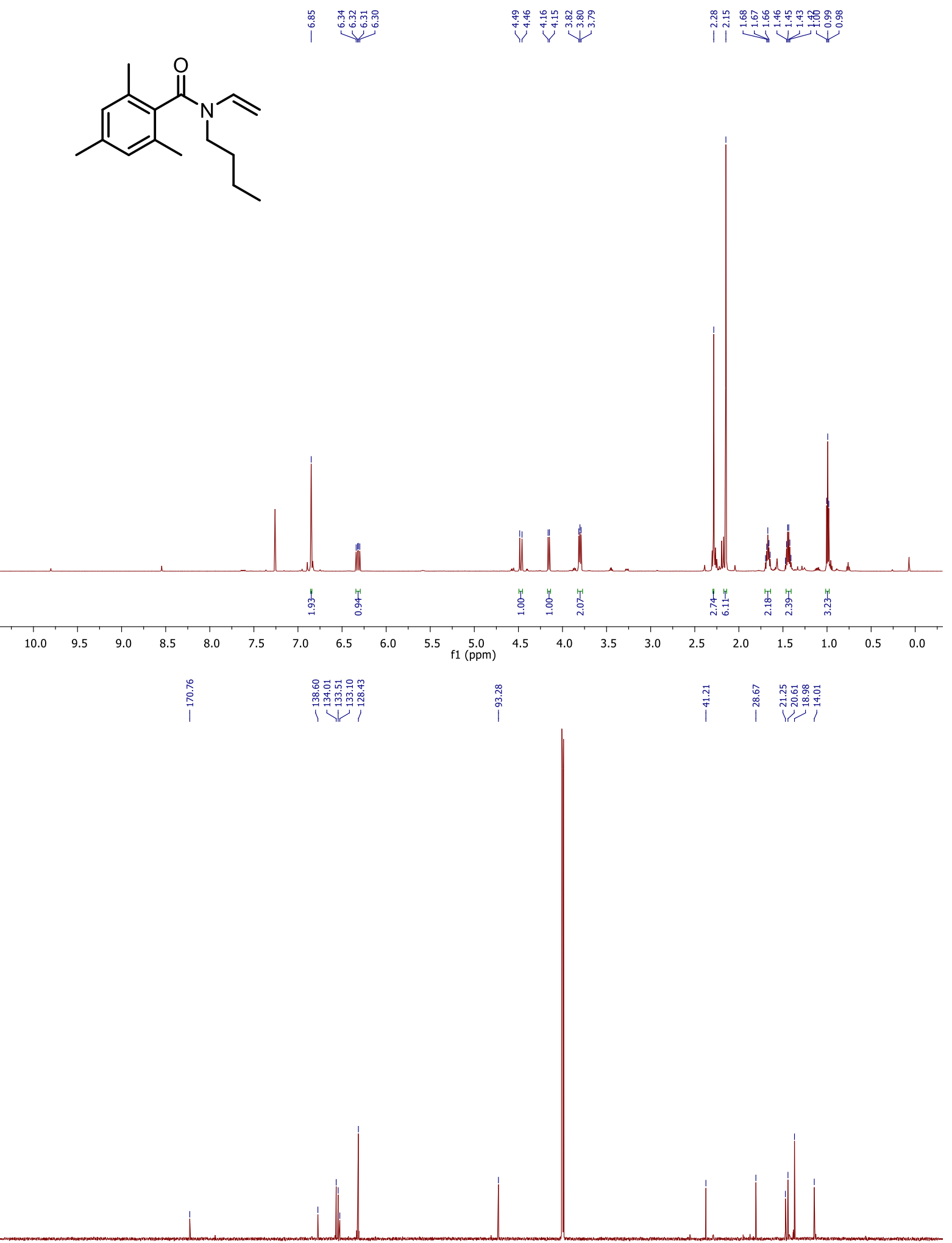

$\begin{array}{lllllllllllllllllllllllllll}210 & 200 & 190 & 180 & 170 & 160 & 150 & 140 & 130 & 120 & 110 & \begin{array}{ll}100 \\ \mathrm{f} 1(\mathrm{ppm})\end{array} & 90 & 80 & 70 & 60 & 50 & 40 & 30 & 20 & 10 & 0 & -10\end{array}$ 
((3S,4R)-3-((Benzo[d][1,3]dioxol-5-yloxy)methyl)-4-(4-fluorophenyl)-3,4-dihydropyridin-1(2H)$\mathrm{yl})$ (mesityl)methanone (2q)

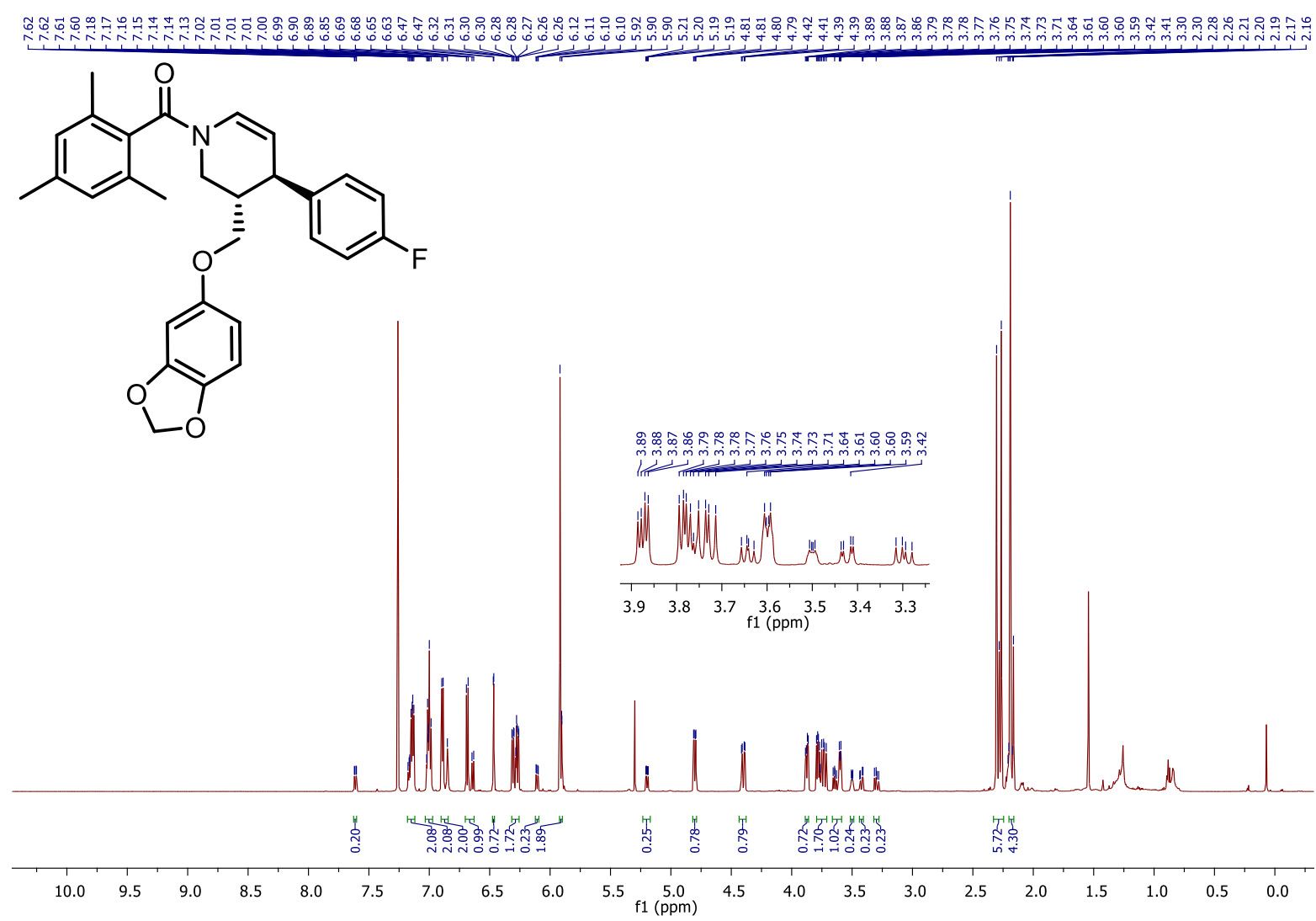

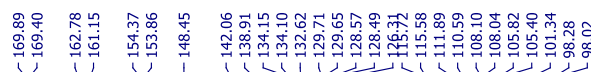

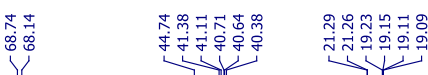

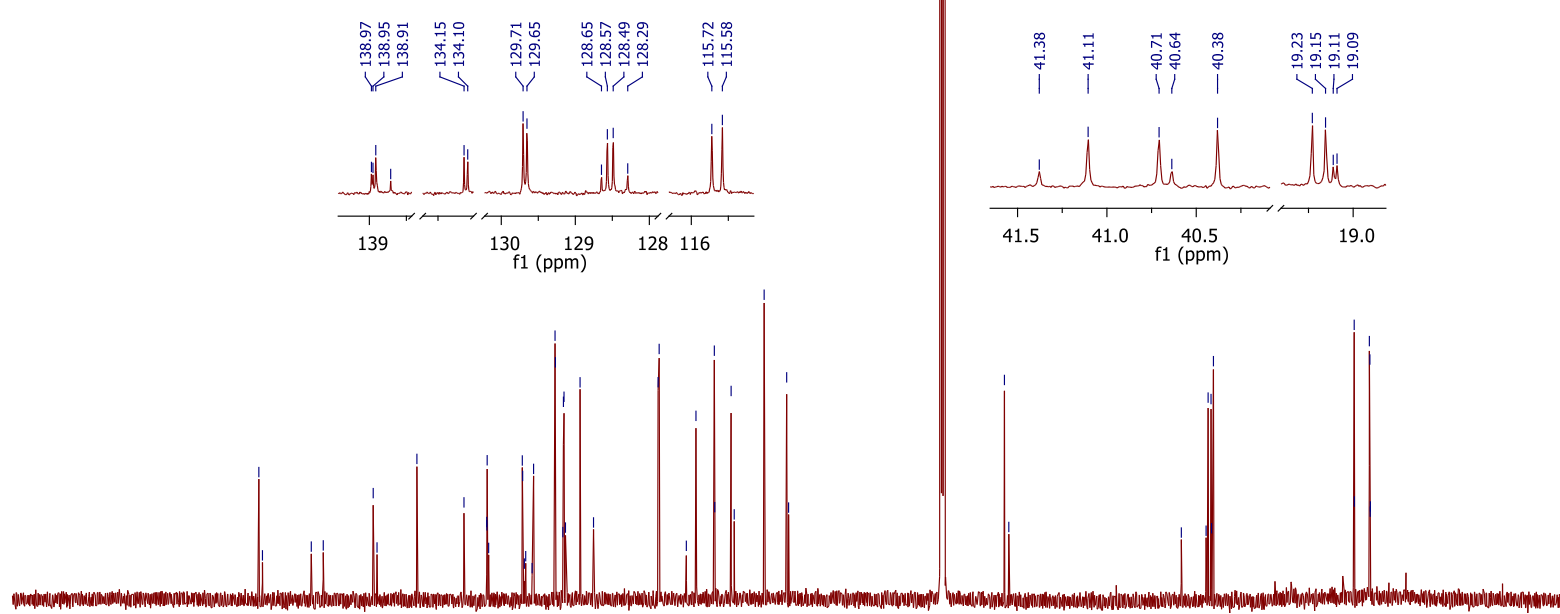

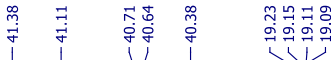

$\begin{array}{lllllllllllllllllllll}200 & 190 & 180 & 170 & 160 & 150 & 140 & 130 & 120 & 110 & \begin{array}{c}100 \\ 100\end{array} & 90 & 80 & 70 & 60 & 50 & 40 & 30 & 20 & 10 & 0\end{array}$ 


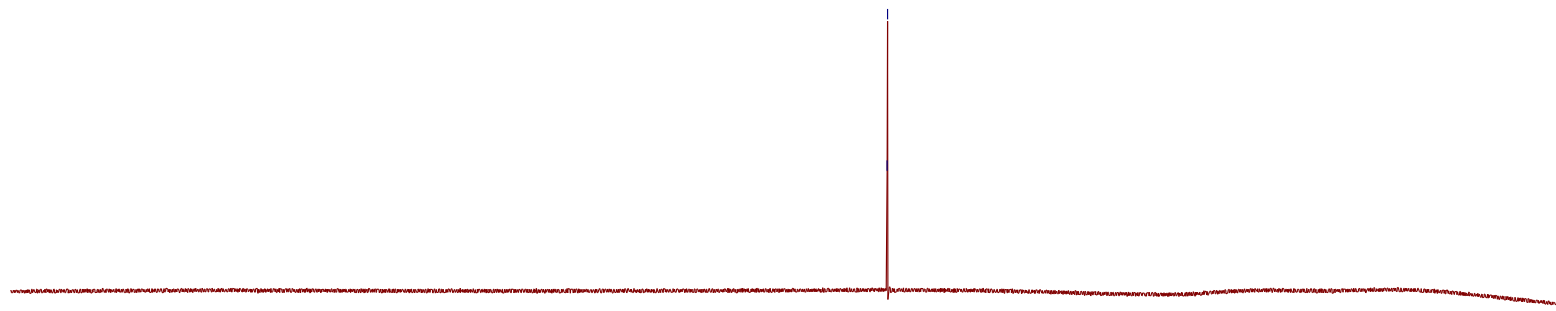

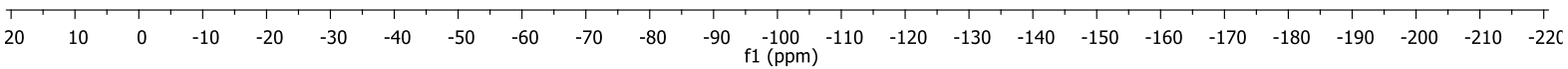


(1-(3,4-Dimethoxybenzyl)-6,7-dimethoxyisoquinolin-2(1H)-yl)(mesityl)methanone (2r)

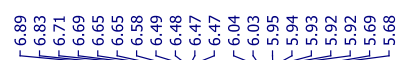

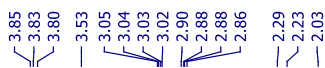<smiles>COc1ccc(CC2c3cc(OC)c(OC)cc3C=CN2C(=O)c2c(C)cc(C)cc2C)cc1OC</smiles>
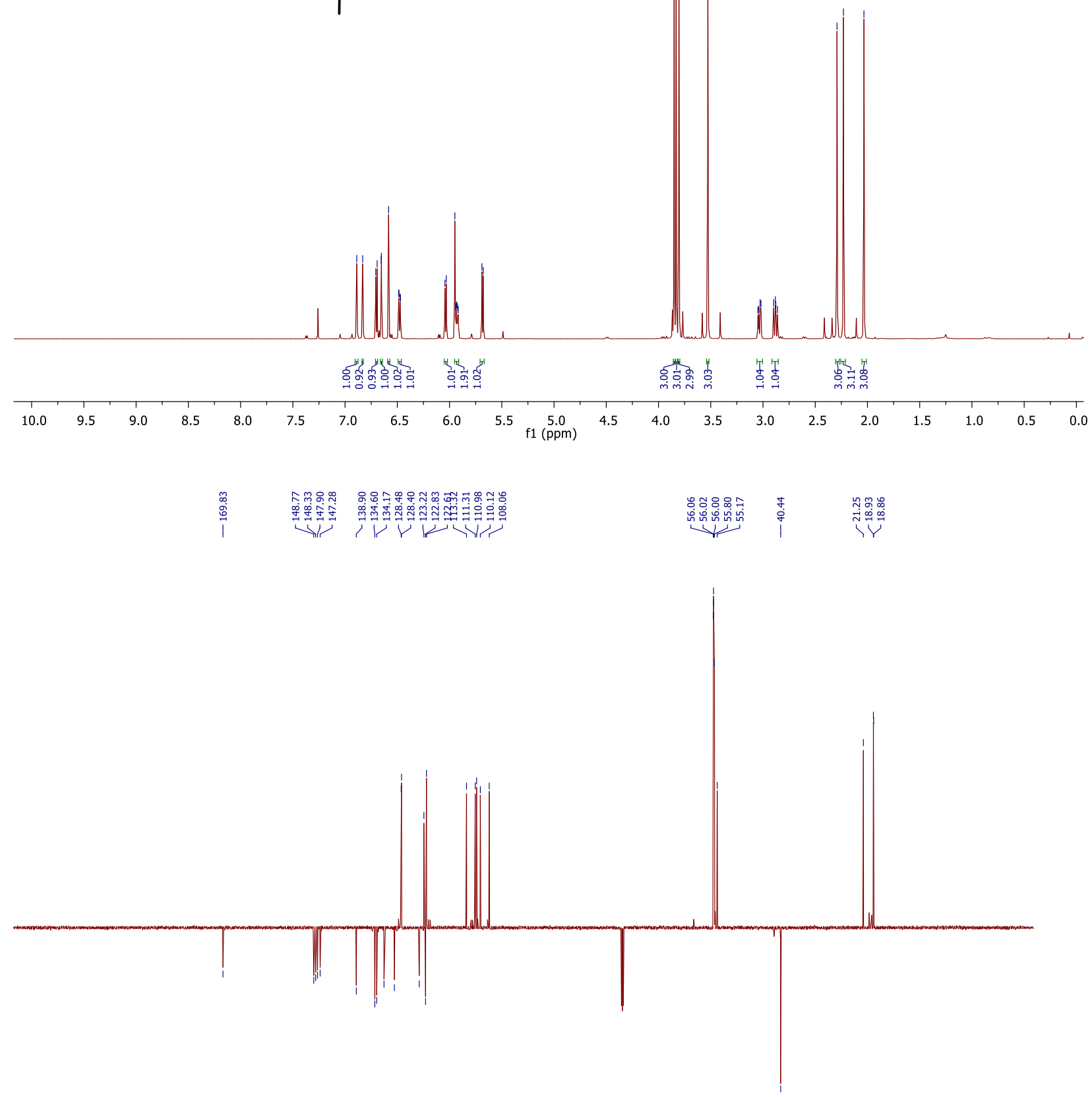

$\begin{array}{lllllllllll}210 & 200 & 190 & 180 & 170 & 160 & 150 & 140 & 130 & 120 & 110 \\ \mathrm{f} 1(\mathrm{ppm})\end{array}$ 


\section{(2,3-Dihydro-1H-pyrrol-1-yl)(2,4,6-triisopropylphenyl)methanone (4a)}

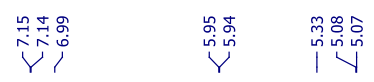

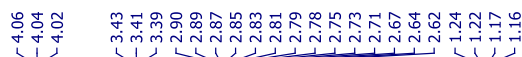<smiles>CC(C)c1cc(C(C)C)c(C(=O)N2C=CCC2)c(C(C)C)c1</smiles>

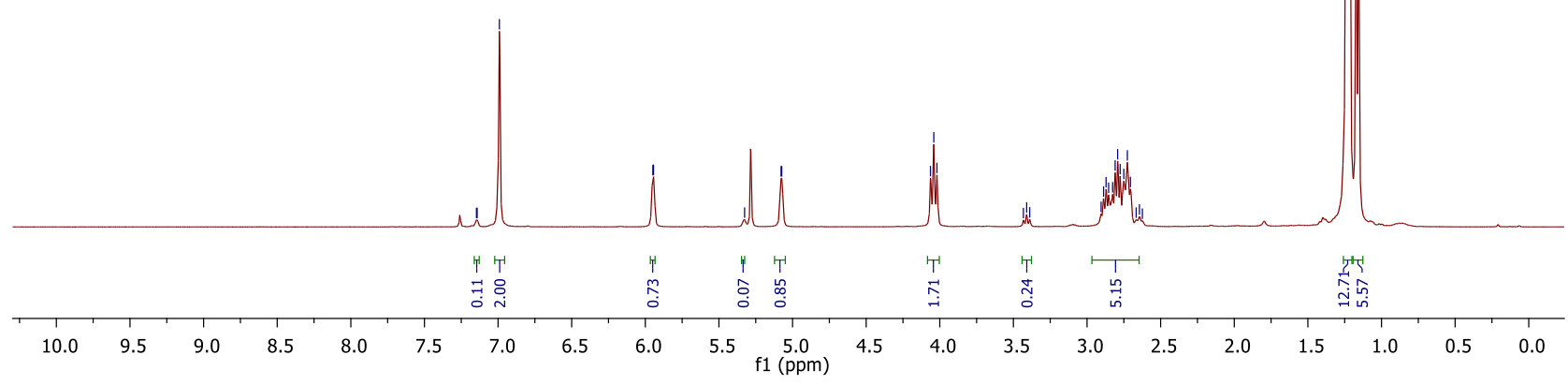
\begin{tabular}{l}
1 \\
\multirow{2}{0}{}
\end{tabular}

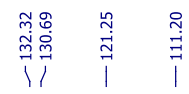
莪

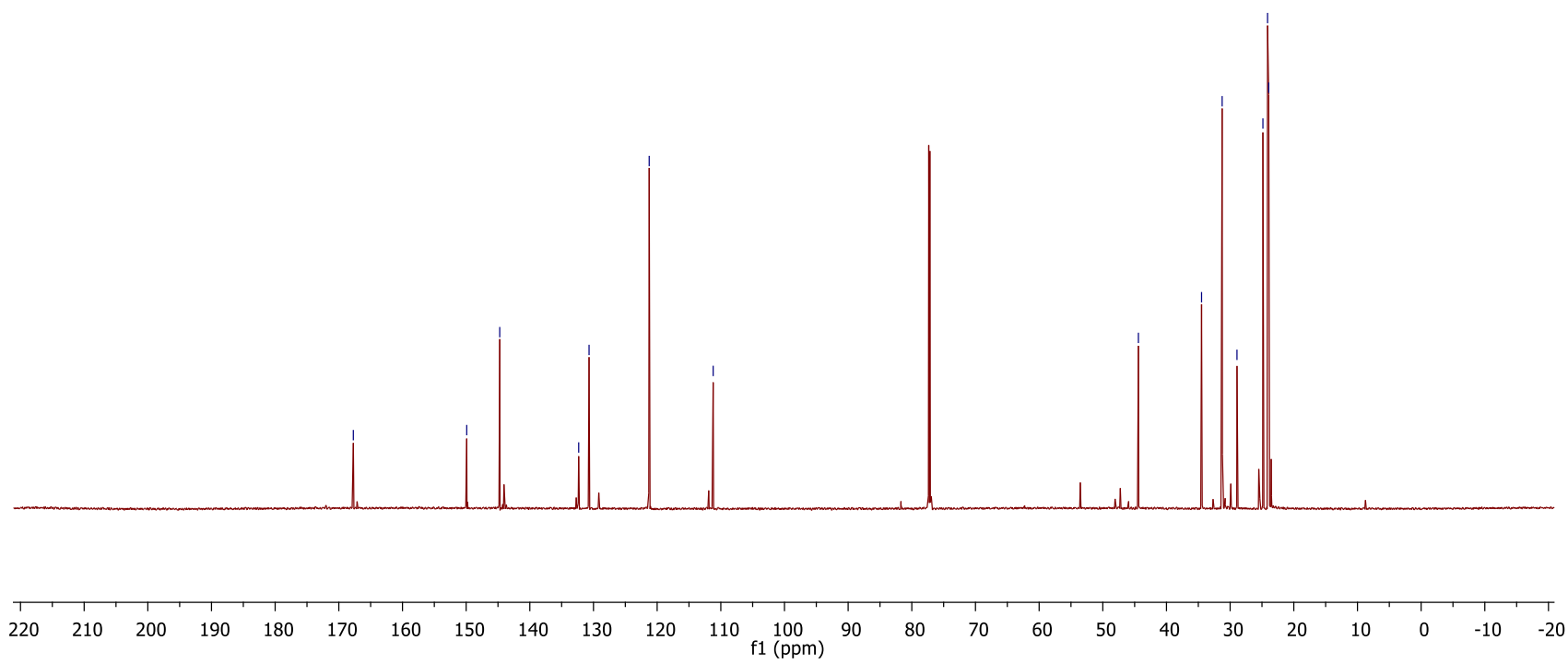


Anthracen-9-yl(2,3-dihydro-1H-pyrrol-1-yl)methanone (4b)

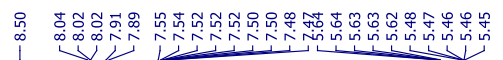

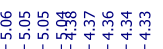

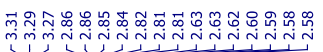<smiles>O=C(c1c2ccccc2cc2ccccc12)N1C=CCC1</smiles>

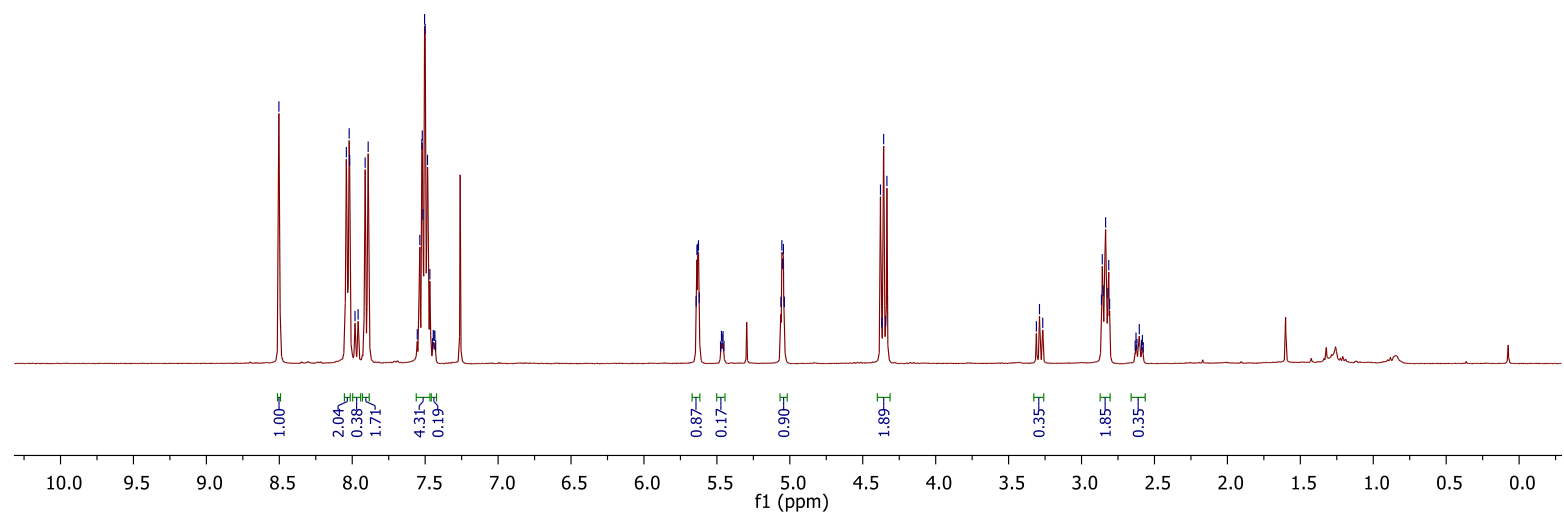

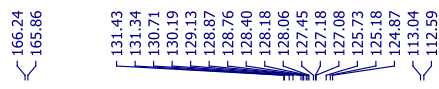

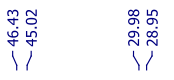

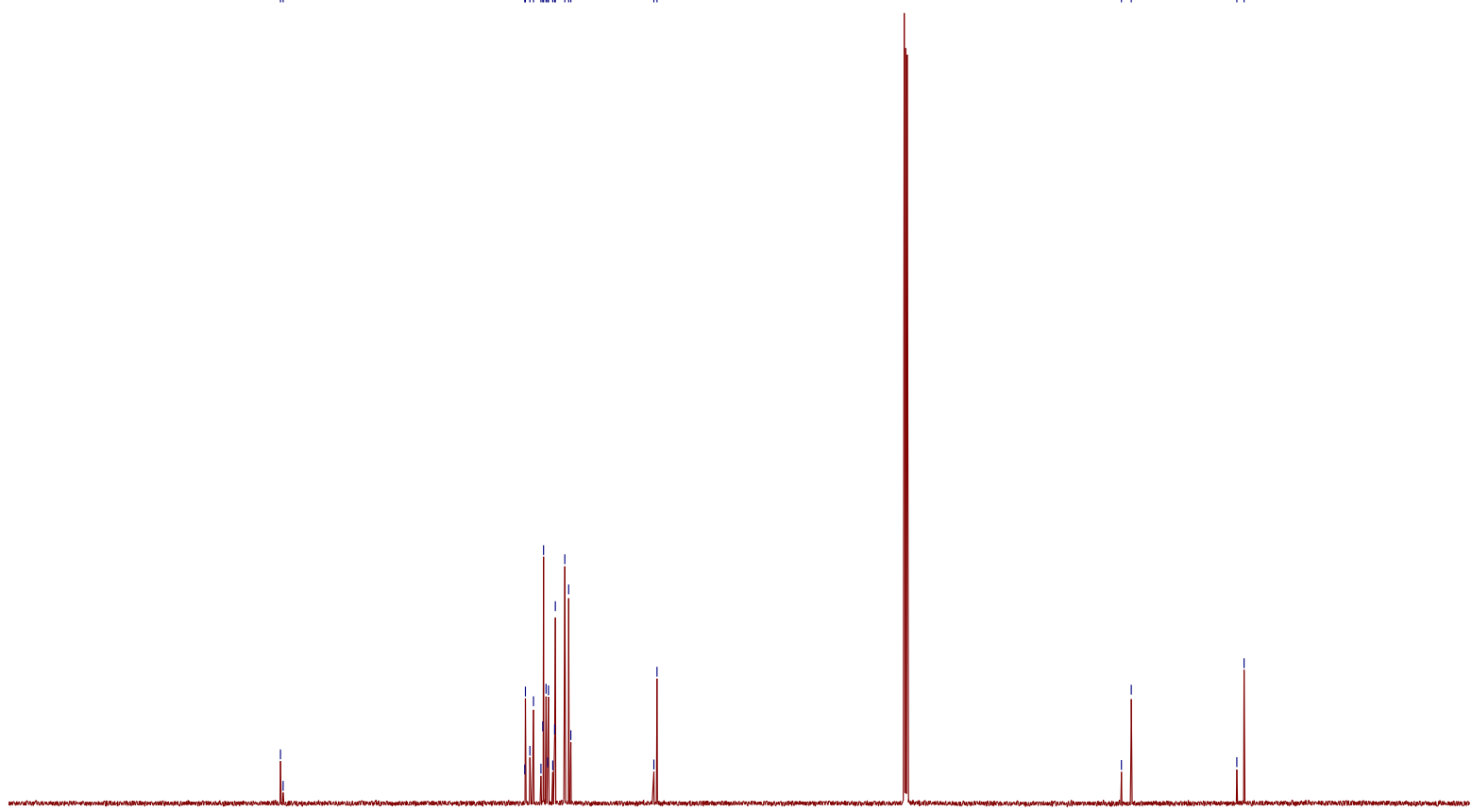

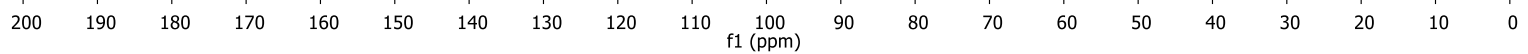


(2,3-Dihydro-1H-pyrrol-1-yl)(phenyl)methanone (4c)

等

$\substack{9 \\ 0 \\ 0 \\ 0}$
0

ำ

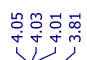

经
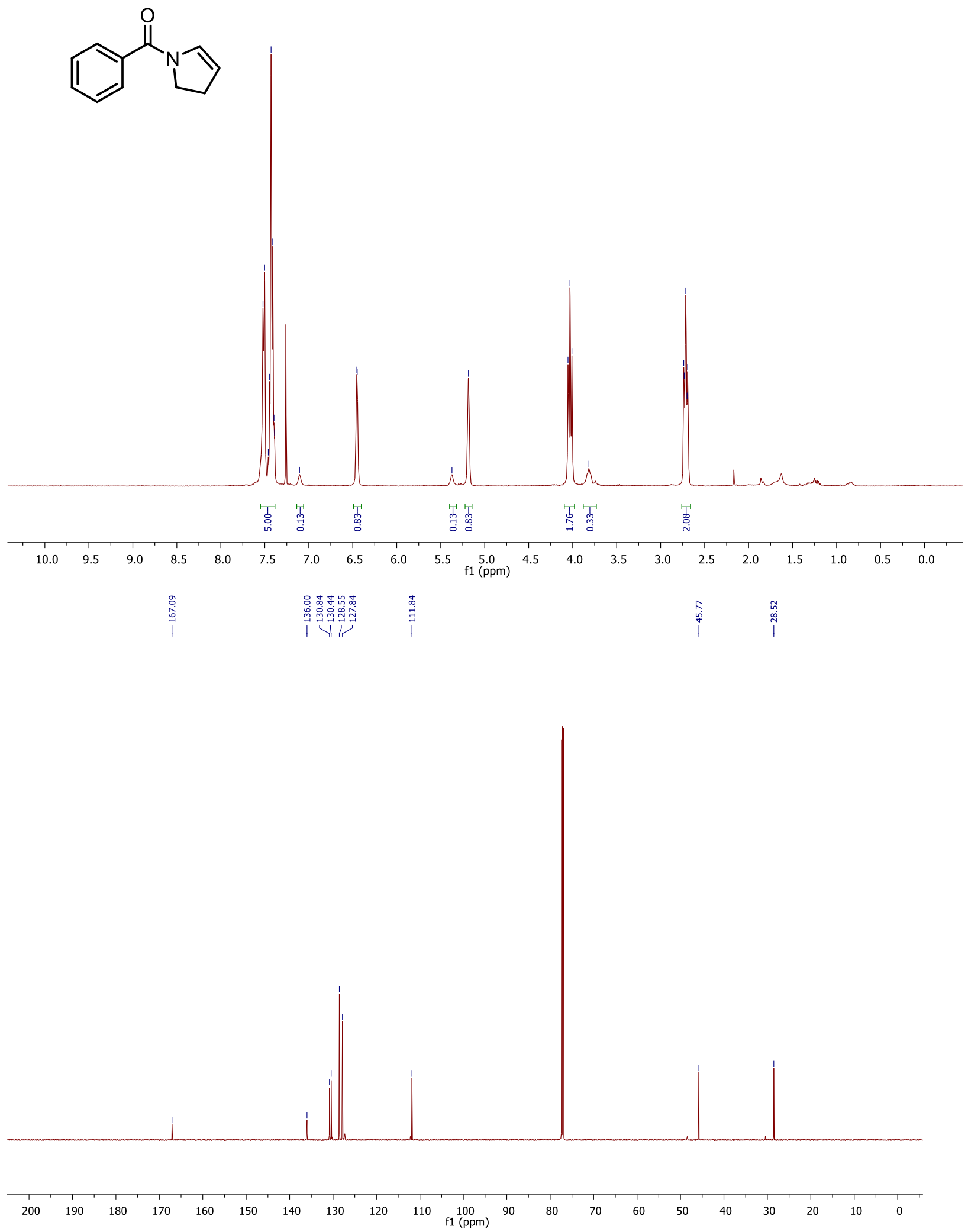

93 


\section{(3,4-Dihydropyridin-1(2H)-yl)(o-tolyl)methanone (4e)}

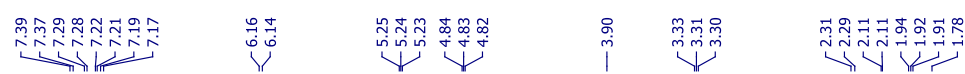

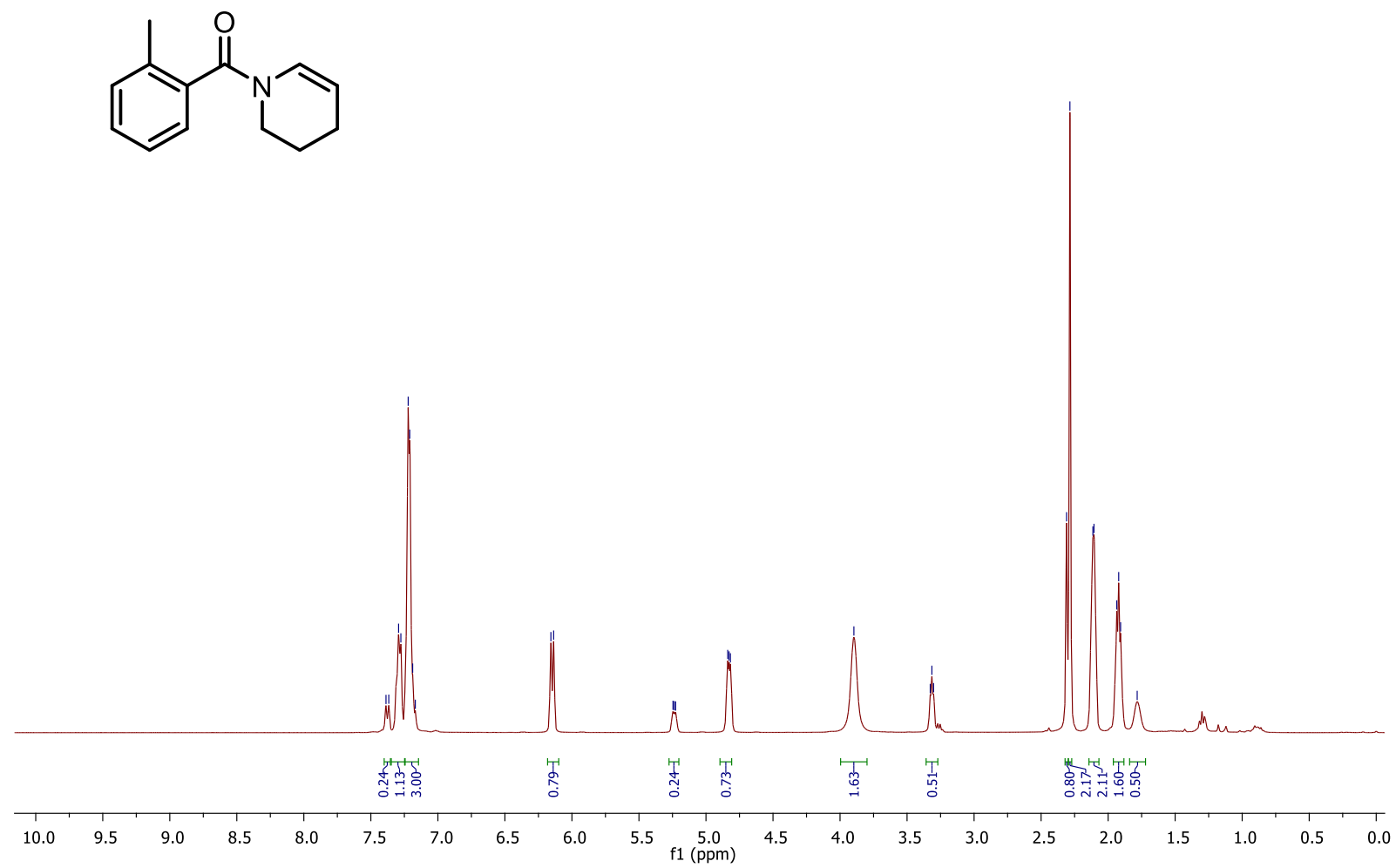

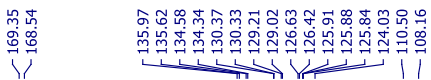

|

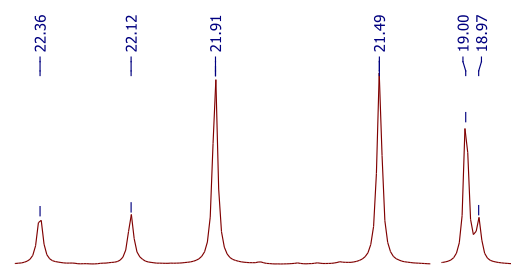

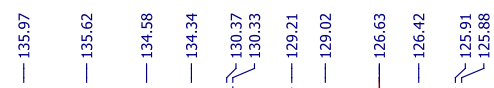
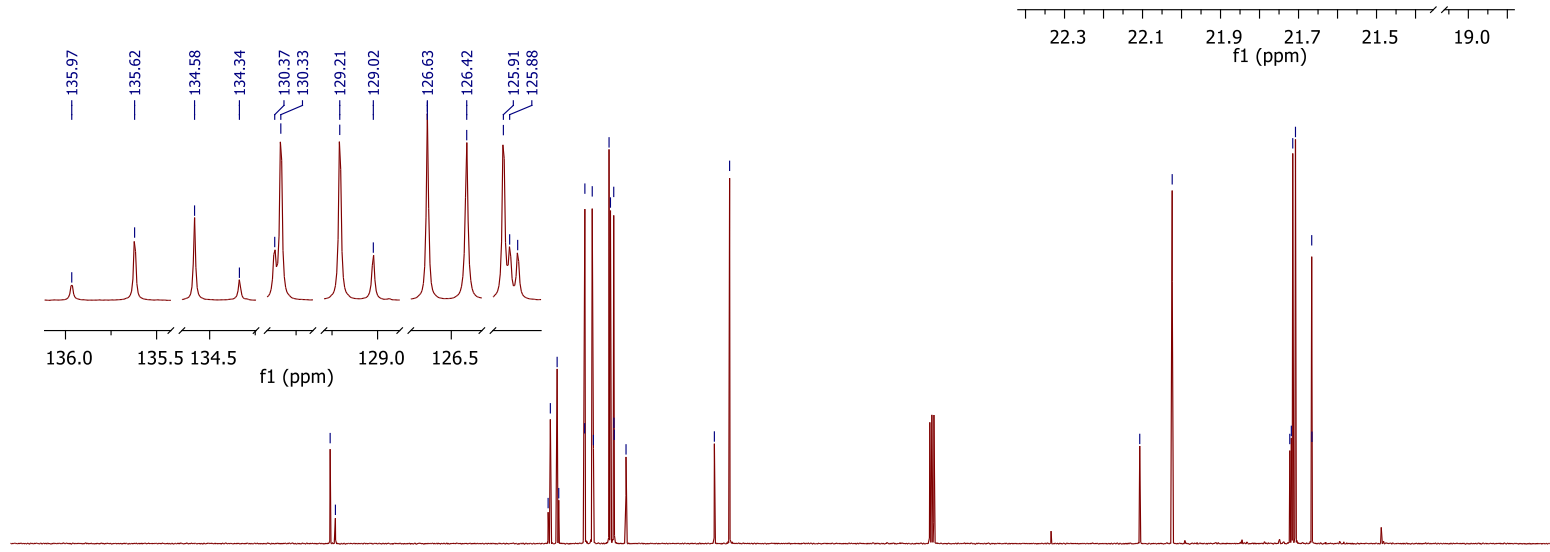

$\begin{array}{lllllllllllllllllllllll}210 & 200 & 190 & 180 & 170 & 160 & 150 & 140 & 130 & 120 & 110 & \begin{array}{c}100 \\ 100\end{array} & 90 & 80 & 70 & 60 & 50 & 40 & 30 & 20 & 10 & 0 & -10\end{array}$ 
(3,4-Dihydropyridin-1(2H)-yl)(p-tolyl)methanone (4f)
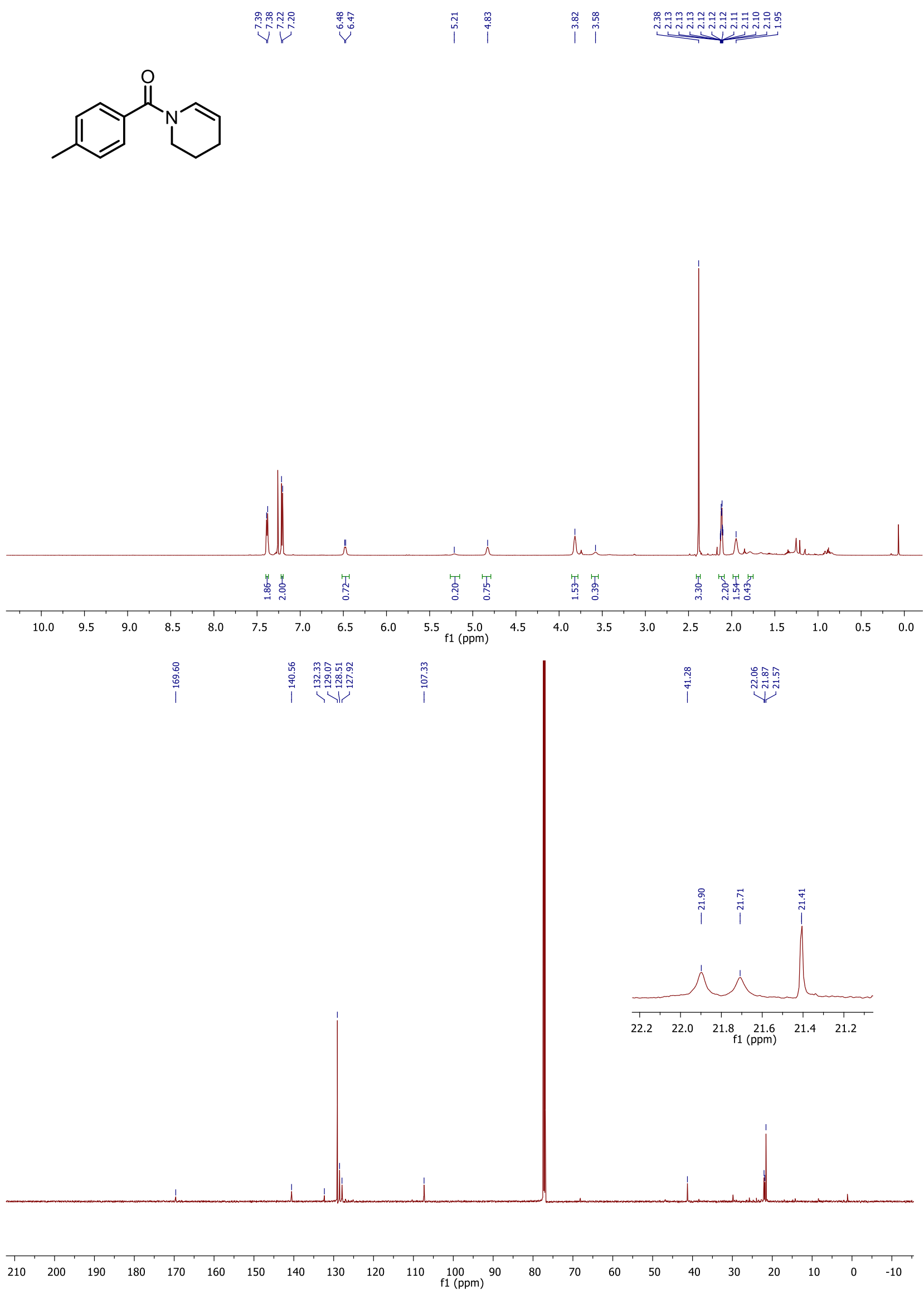

95 


\section{(3,4-Dihydropyridin-1(2H)-yl)(2-methoxyphenyl)methanone (4g)}

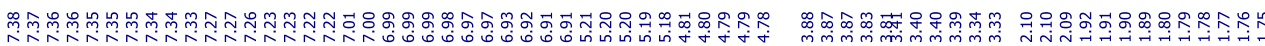<smiles>COc1ccccc1C(=O)N1C=CCCC1</smiles>
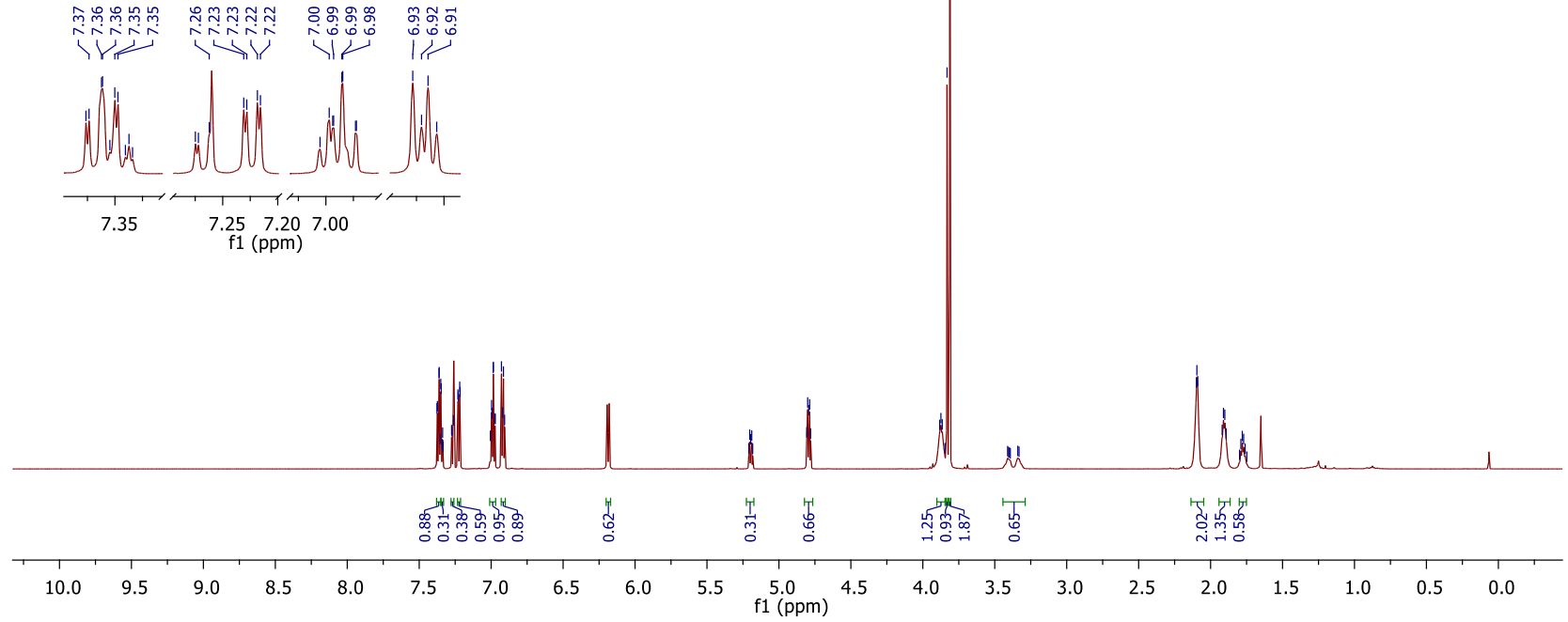
V V
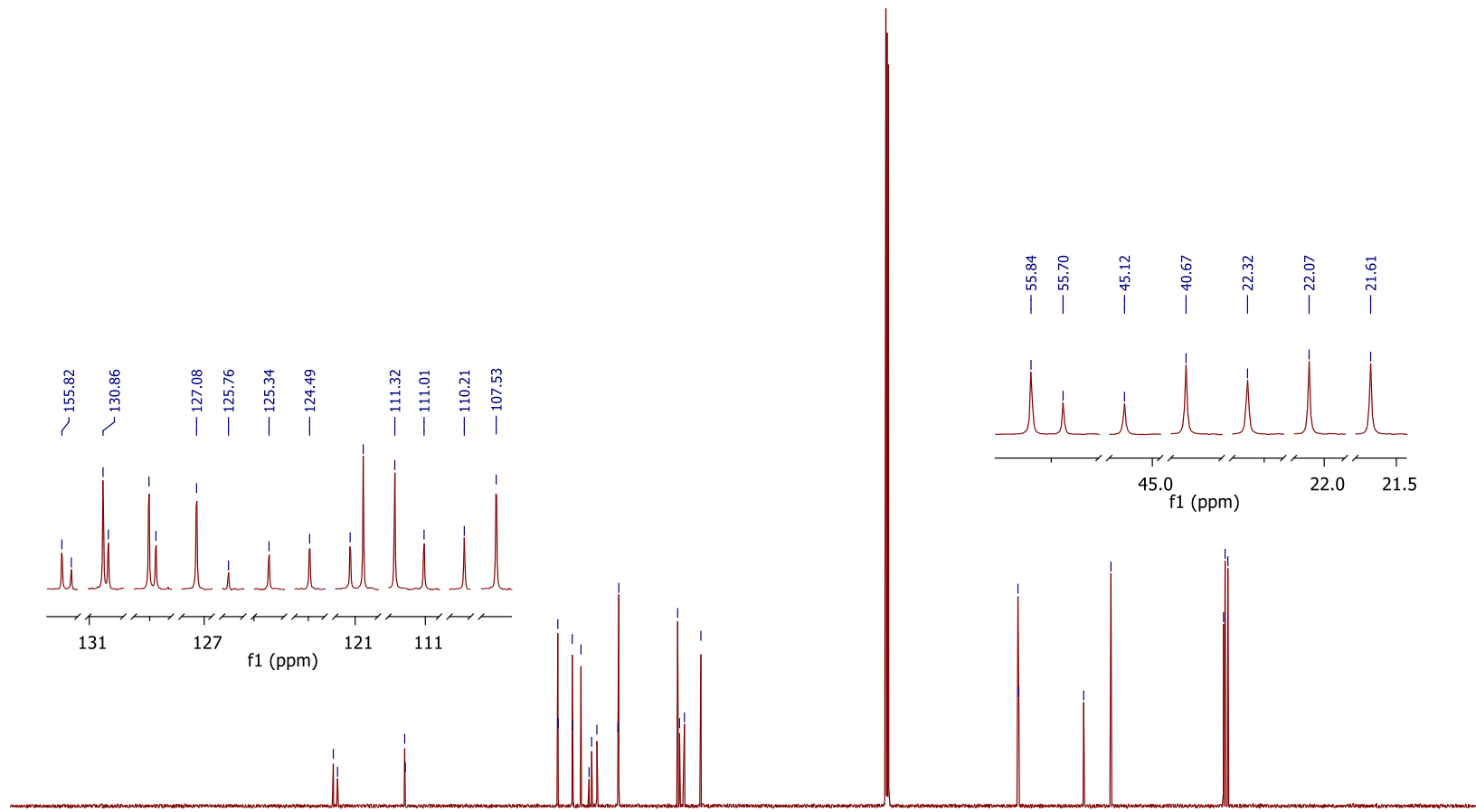

$\begin{array}{lllllllllllllllllllllllllllllllll}220 & 210 & 200 & 190 & 180 & 170 & 160 & 150 & 140 & 130 & 120 & 110 & 100 & 90 & 80 & 70 & 60 & 50 & 40 & 30 & 20 & 10 & 0 & -10\end{array}$ 
(3,4-Dihydropyridin-1(2H)-yl)(3-methoxyphenyl)methanone (4h)

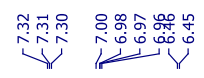

界等要

影管

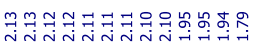
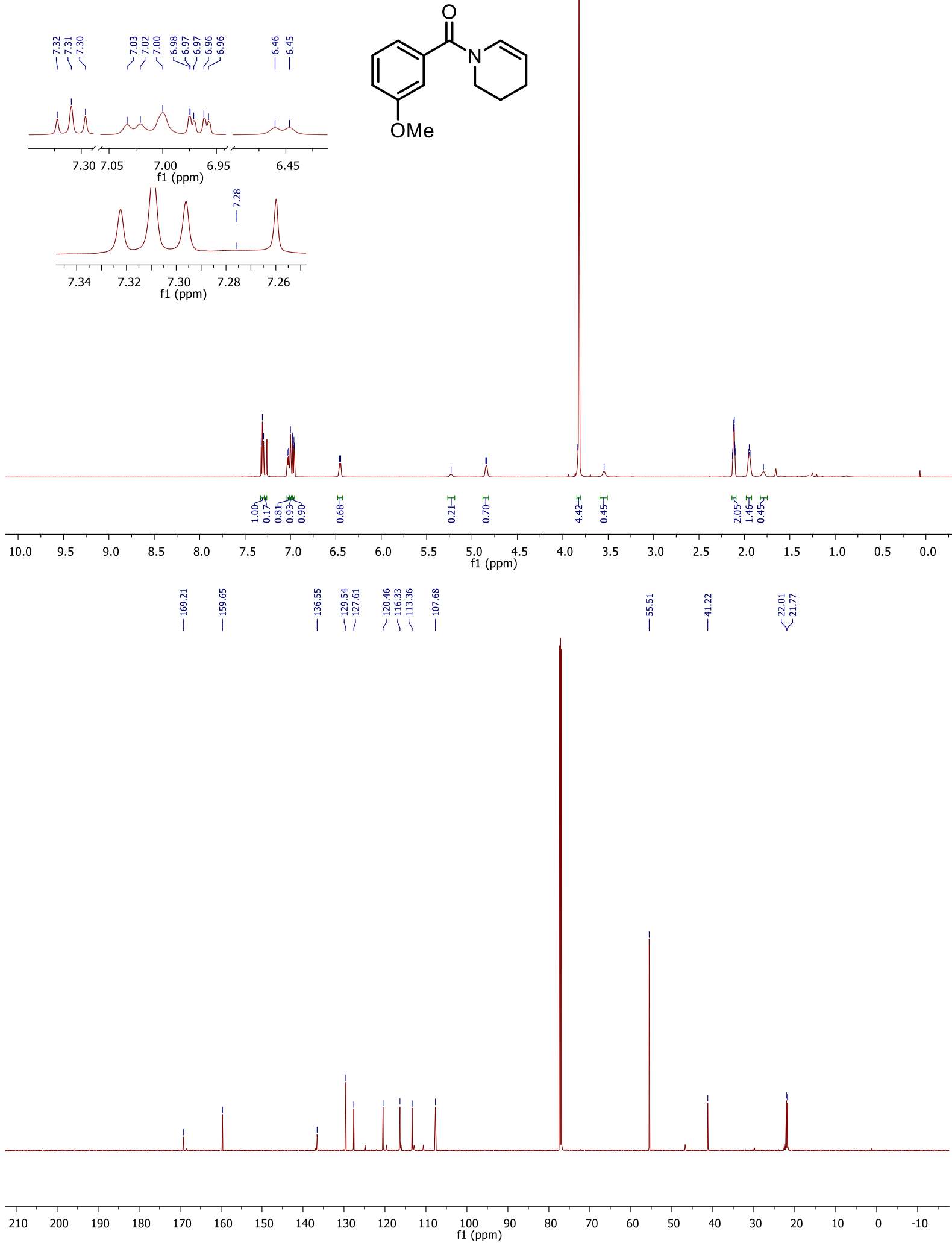

97 
(3,4-Dihydropyridin-1(2H)-yl)(4-methoxyphenyl)methanone (4i)
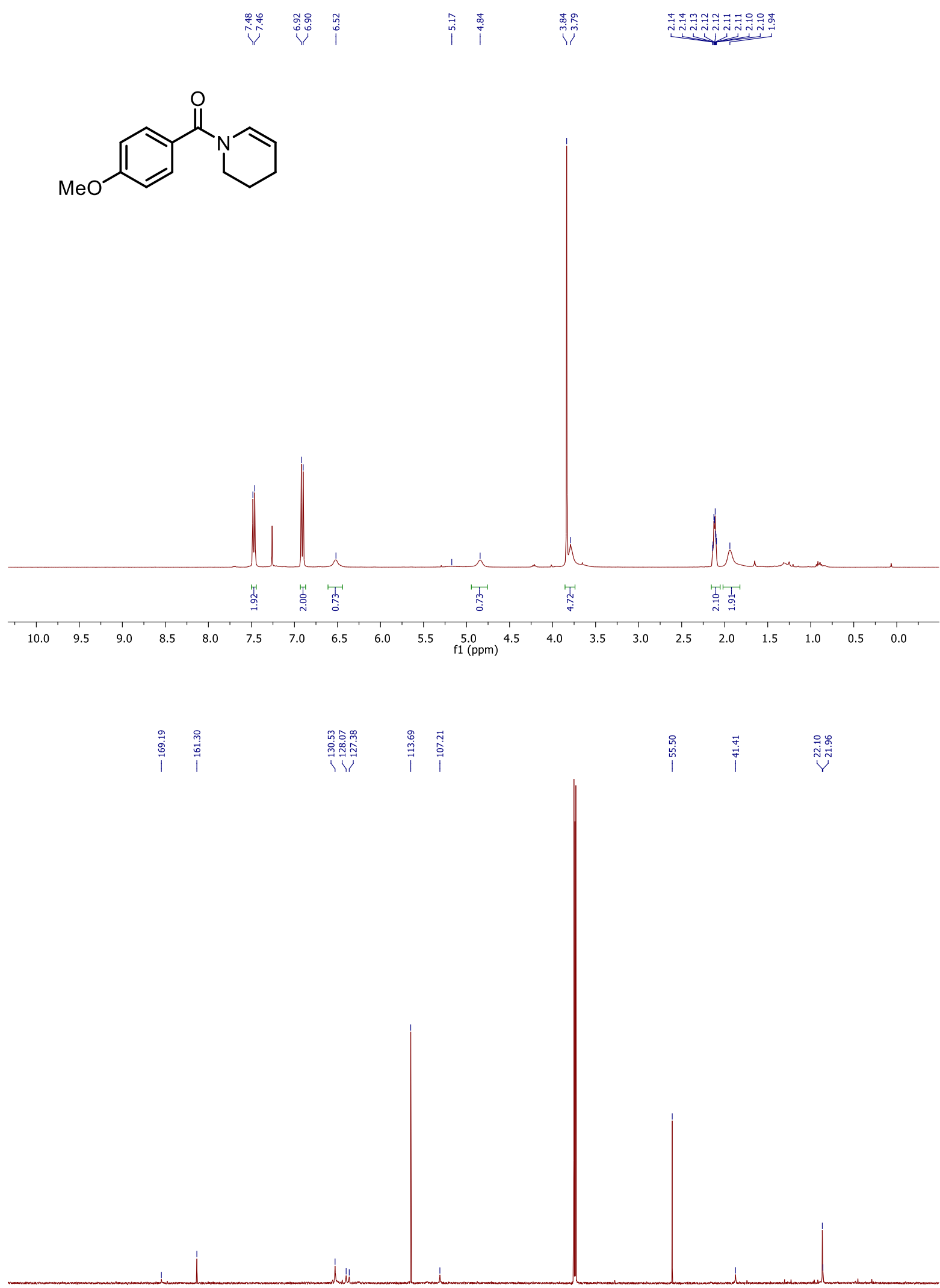

$\begin{array}{lllllllllllllllllllllll}200 & 190 & 180 & 170 & 160 & 150 & 140 & 130 & 120 & 110 & 100 & 90 & 80 & 70 & 60 & 50 & 40 & 30 & 20 & 10 & 0\end{array}$ 
(2,3-Dihydro-1H-pyrrol-1-yl)(3,4,5-trimethoxyphenyl)methanone (4j)
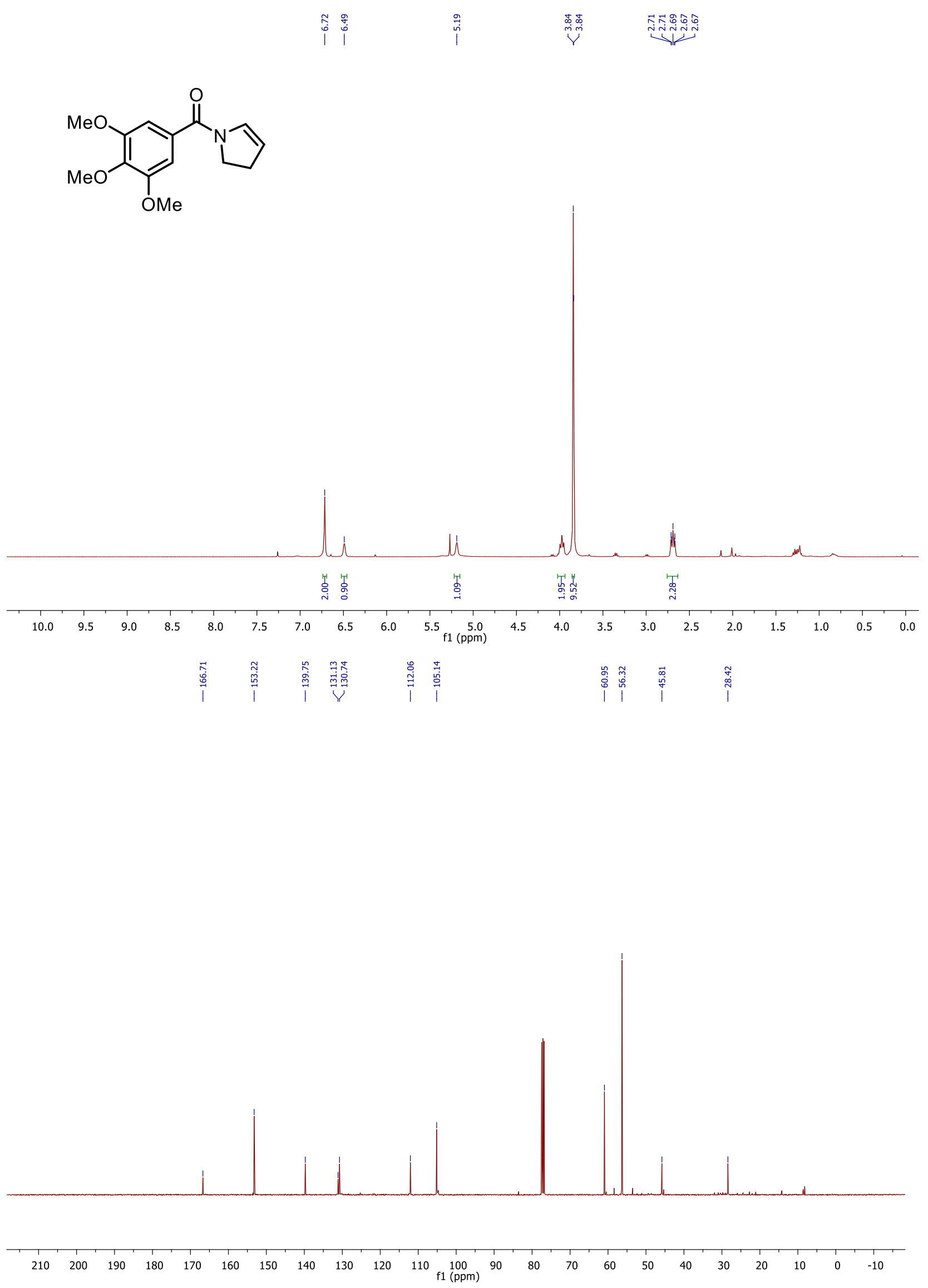

99 
(3,4-Dihydropyridin-1(2H)-yl)(3,4,5-trimethoxyphenyl)methanone (4k)
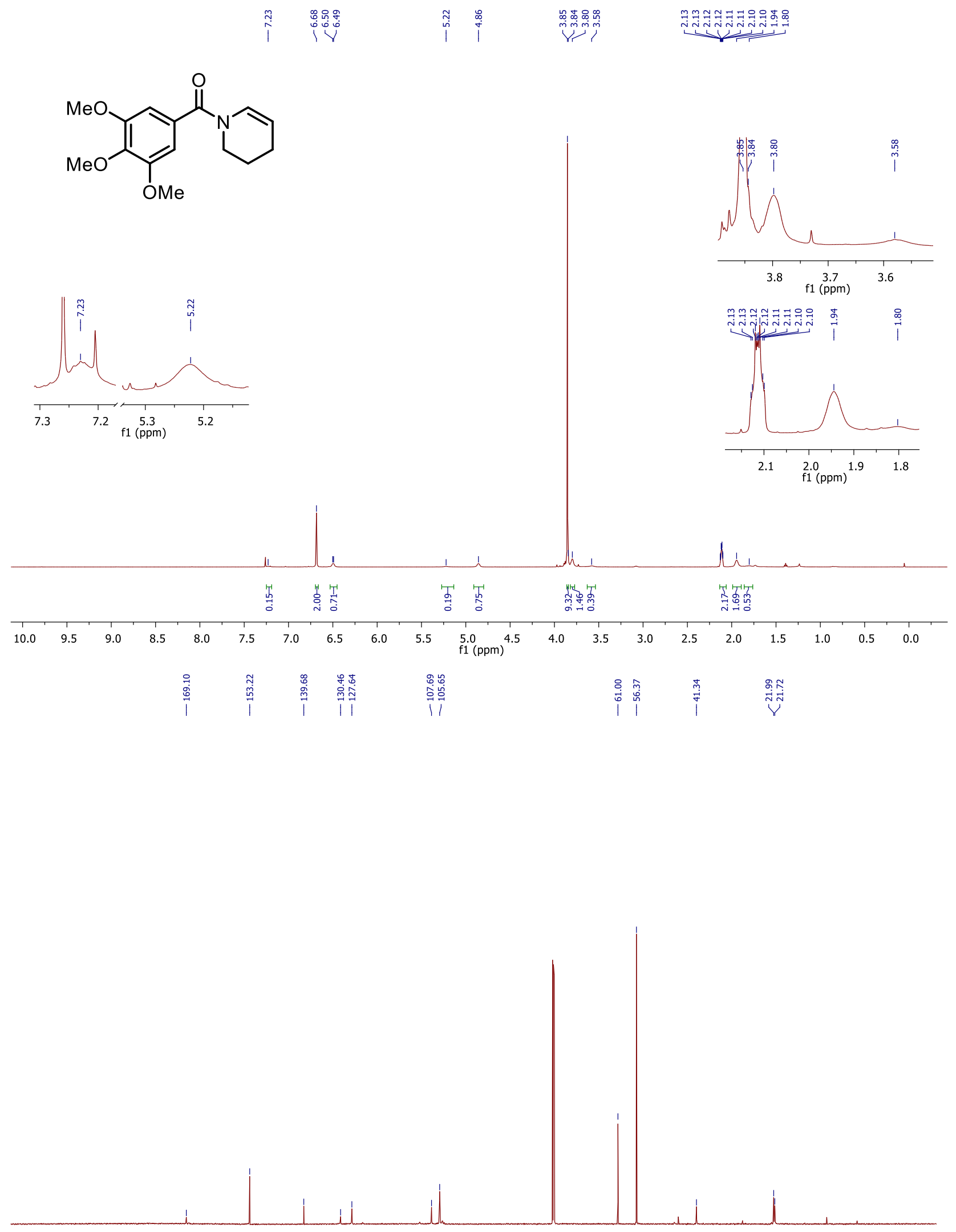

$\begin{array}{lllllllllllllllllllllllllllll}210 & 200 & 190 & 180 & 170 & 160 & 150 & 140 & 130 & 120 & 110 & 100 & 90 & 80 & 70 & 60 & 50 & 40 & 30 & 20 & 10 & 0 & -10\end{array}$ 
Benzo[d][1,3]dioxol-5-yl(3,4-dihydropyridin-1(2H)-yl)methanone (4I)

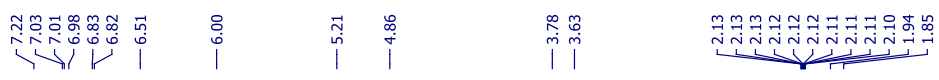<smiles>O=C(c1ccc2c(c1)OCO2)N1C=CCCC1</smiles>
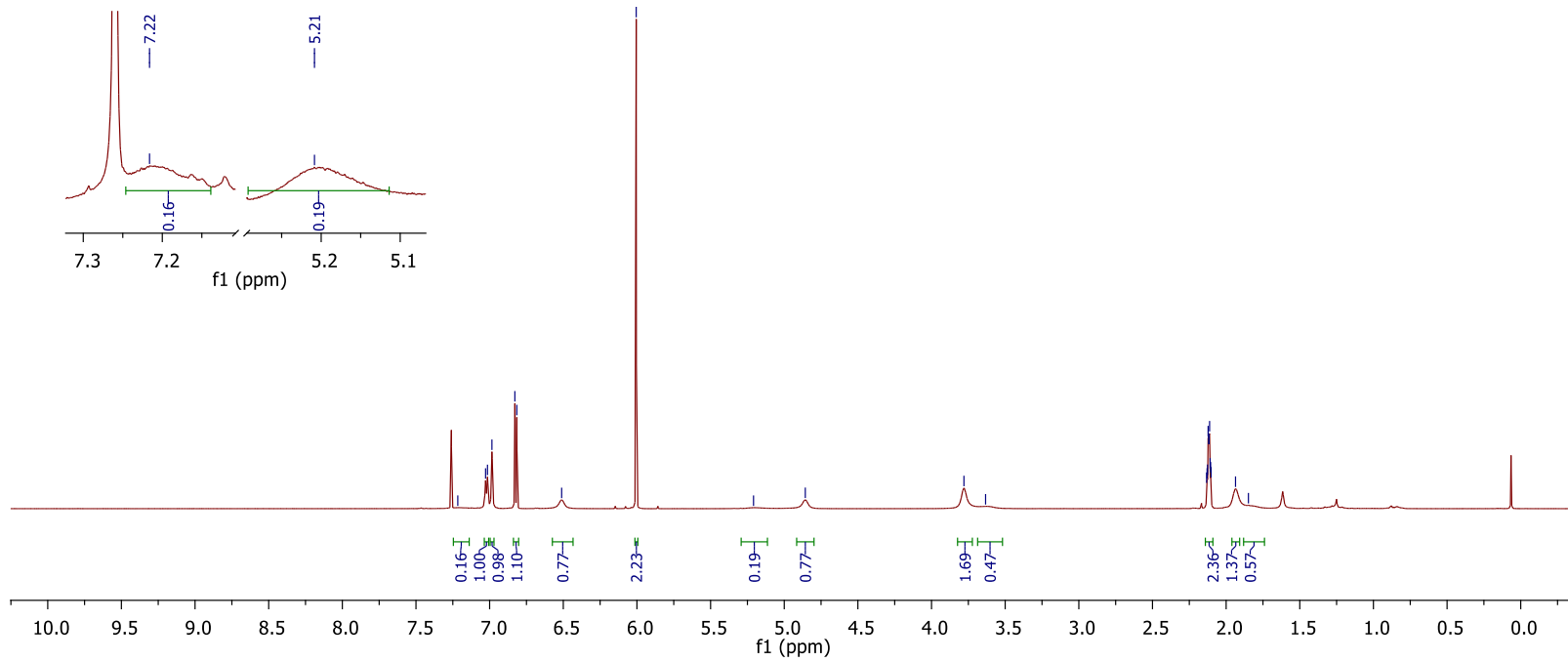

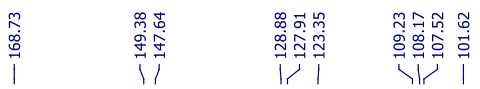

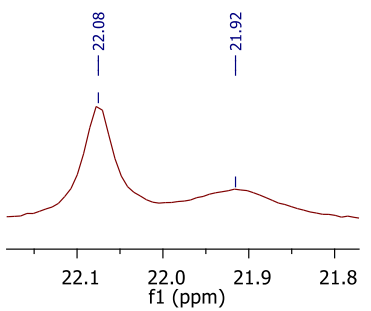

$\begin{array}{llllllllllllllllllllllll}220 & 210 & 200 & 190 & 180 & 170 & 160 & 150 & 140 & 130 & 120 & 110 & 100 & 90 & 80 & 70 & 60 & 50 & 40 & 30 & 20 & 10 & 0 & -10\end{array}$ 
<smiles>COc1ccc(Br)c(C(=O)N2C=CCC2)c1</smiles>

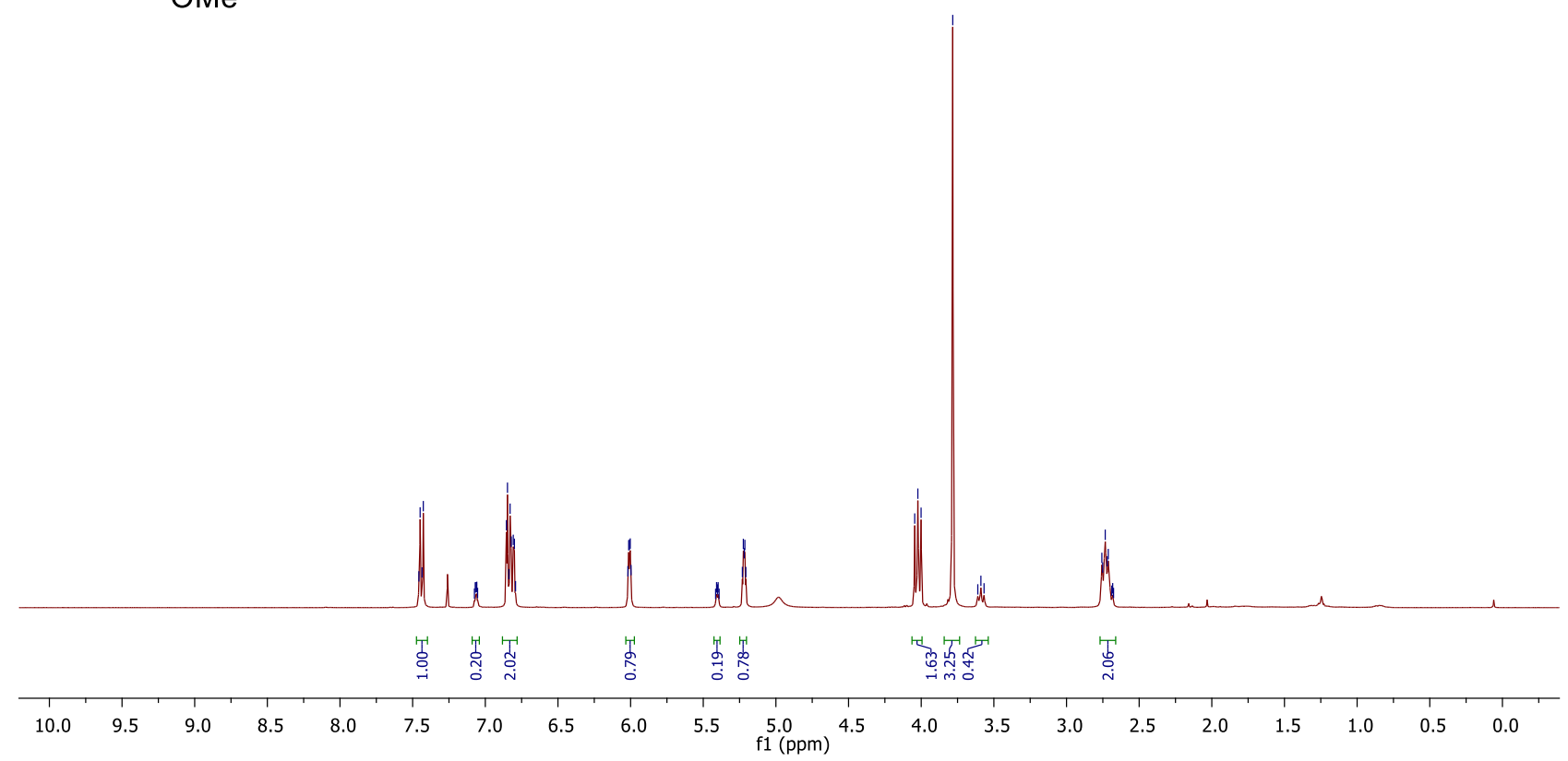

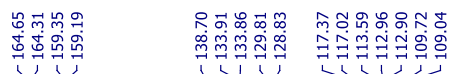
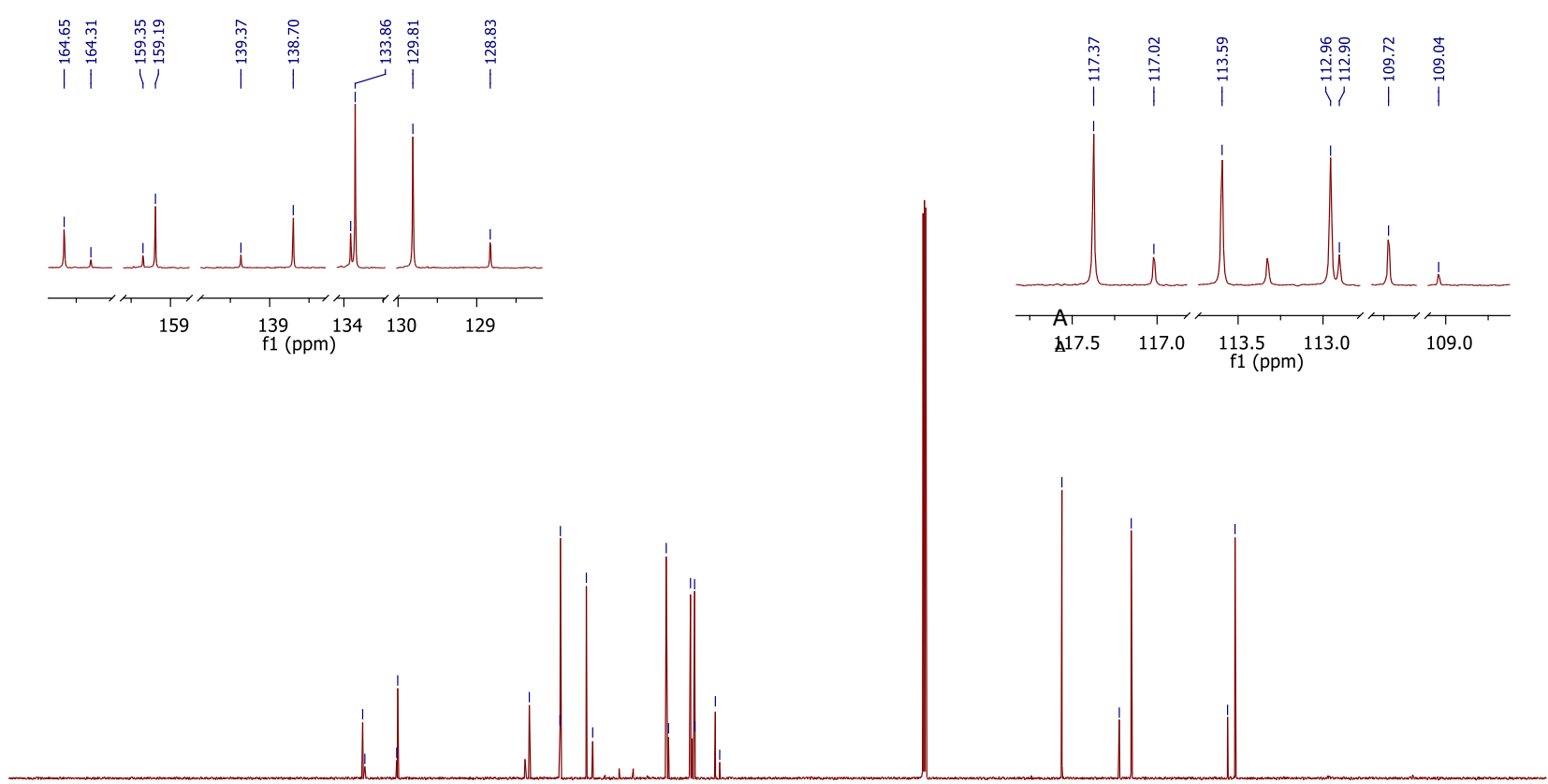

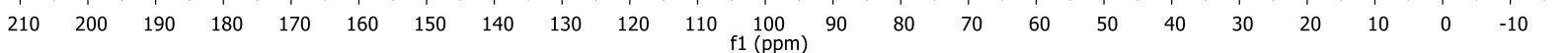


(2,3-Dihydro-1H-pyrrol-1-yl)(4-fluorophenyl)methanone (4n)

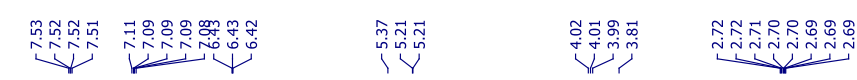

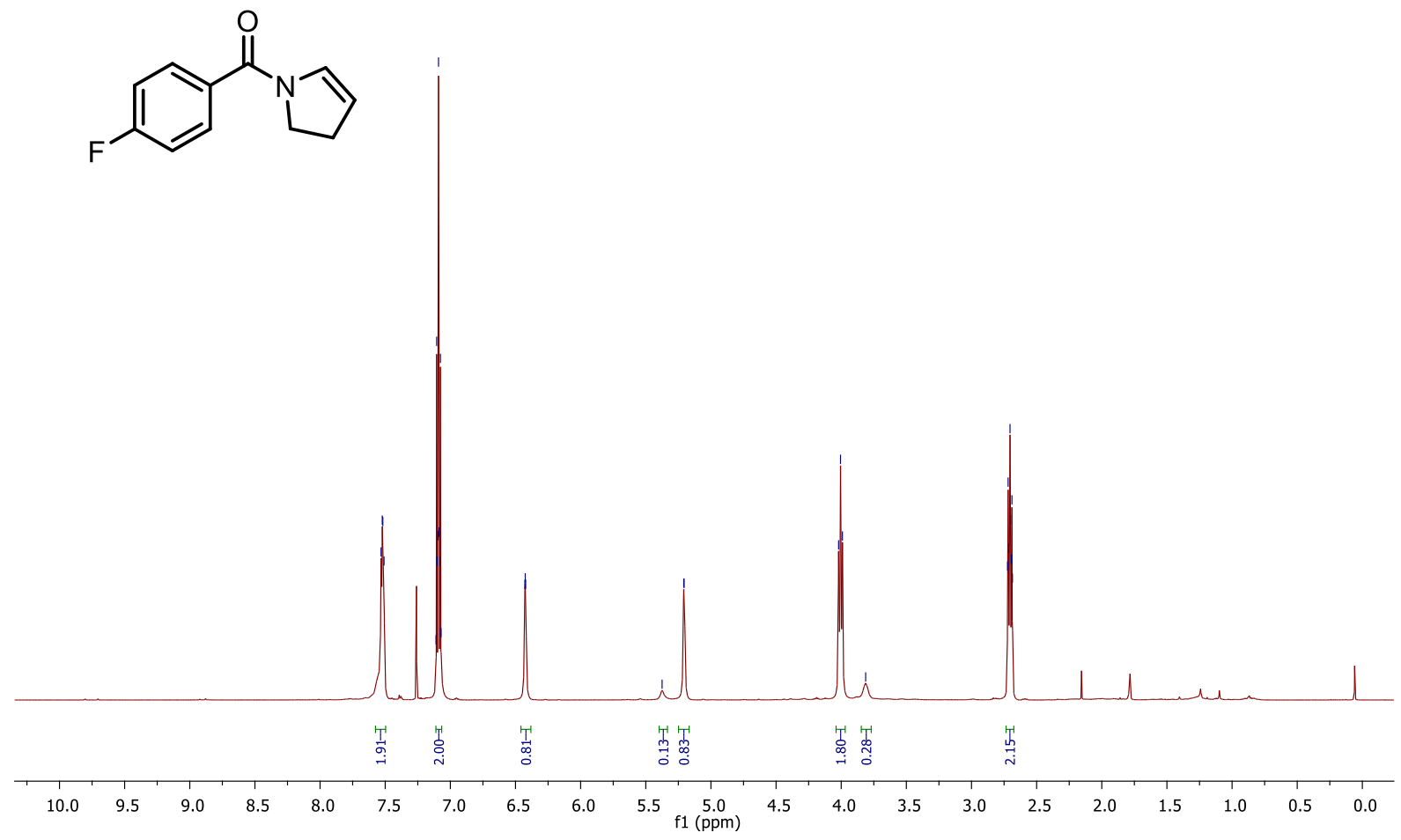

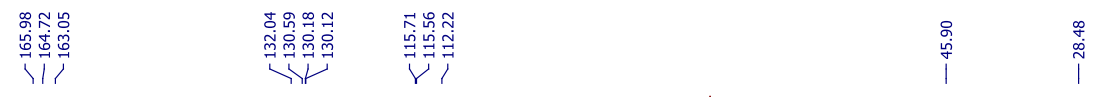

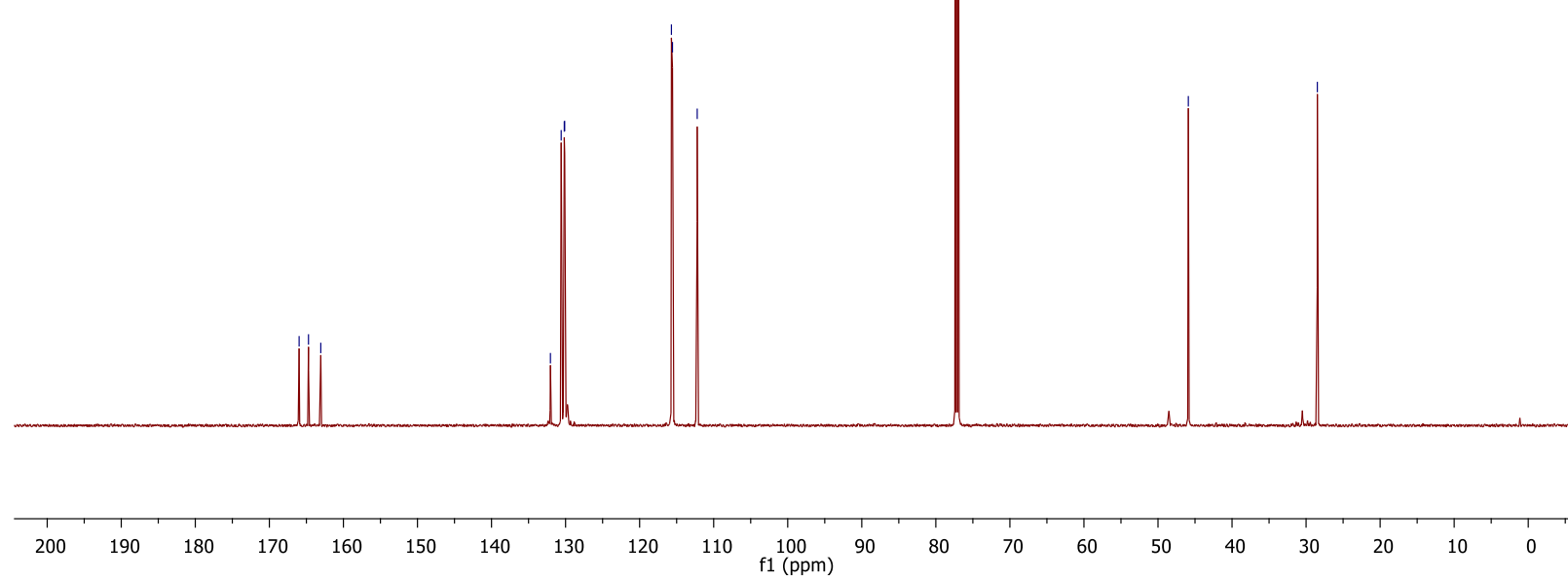




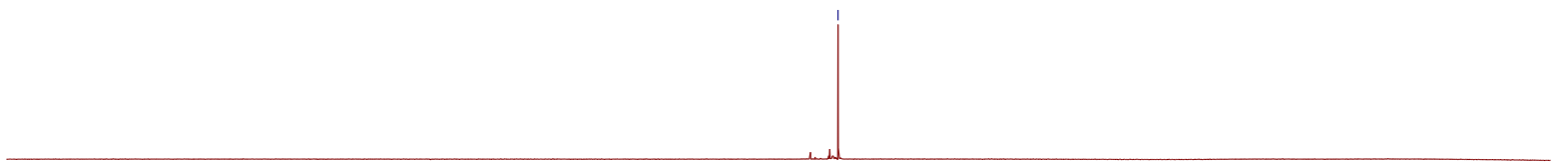

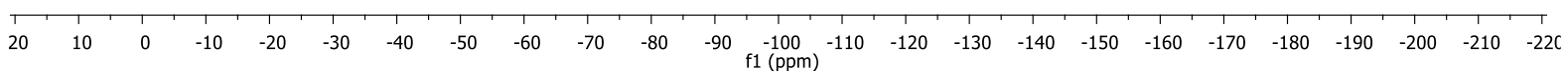


(3-Chlorophenyl)(2,3-dihydro-1H-pyrrol-1-yl)methanone (4o)

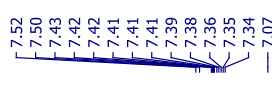

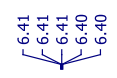

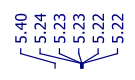

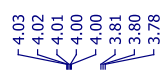

ํำ
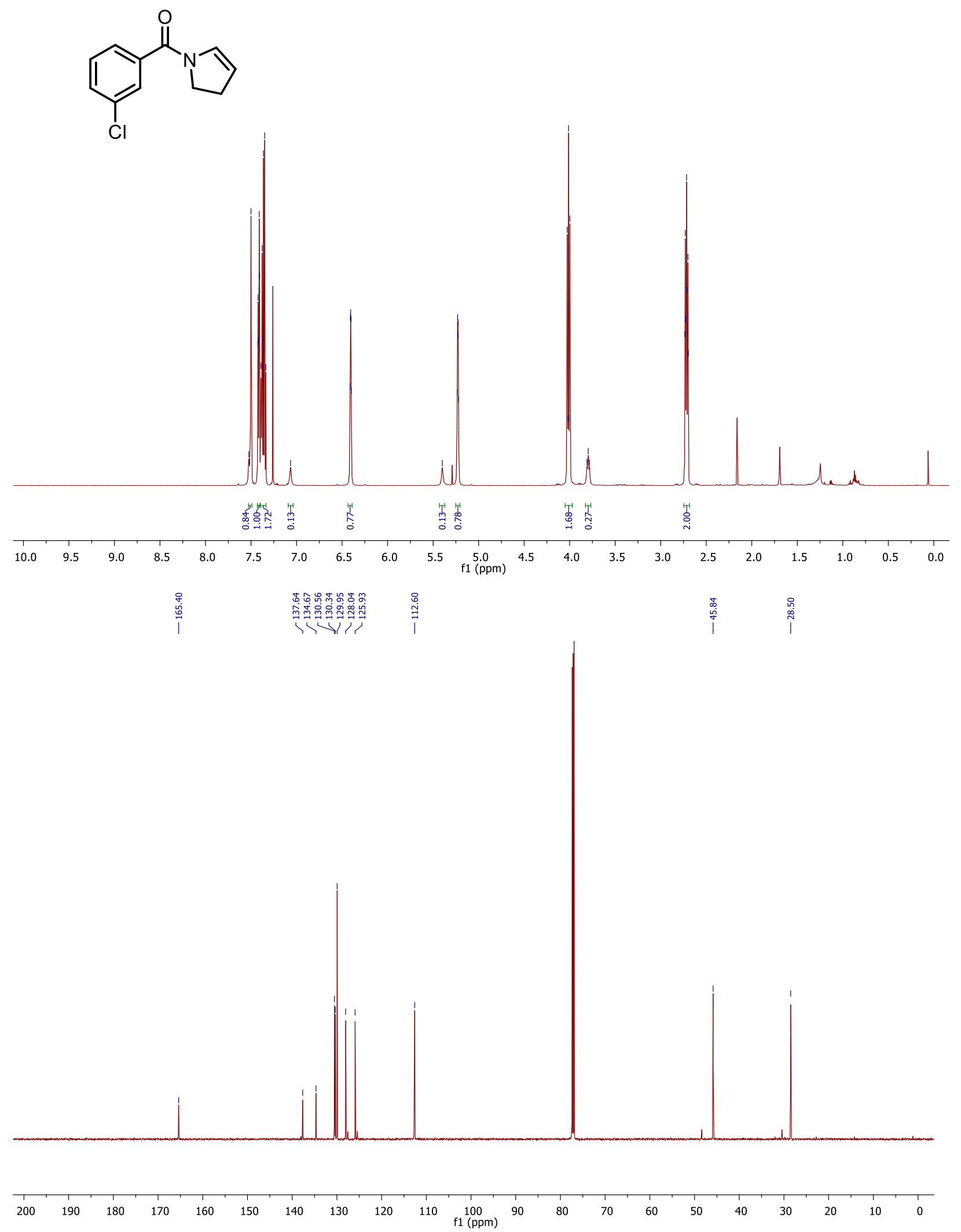

105 


\section{(2,3-Dihydro-1H-pyrrol-1-yl)(4-vinylphenyl)methanone (4p)}

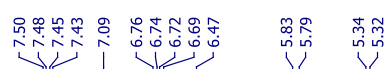

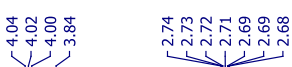<smiles>C=Cc1ccc(C(=O)N2C=CCC2)cc1</smiles>

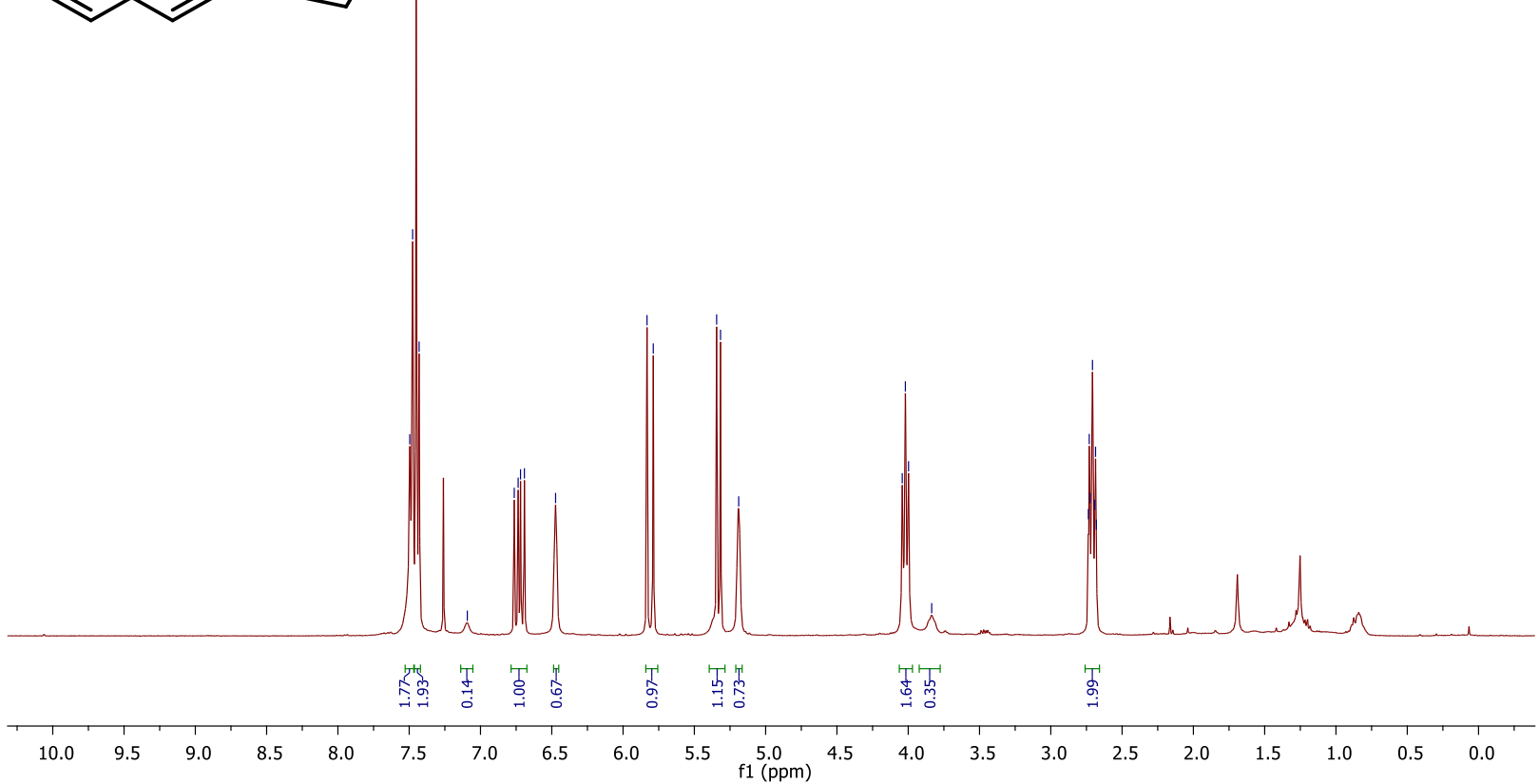

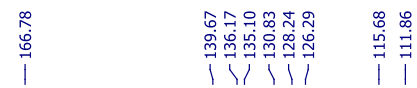

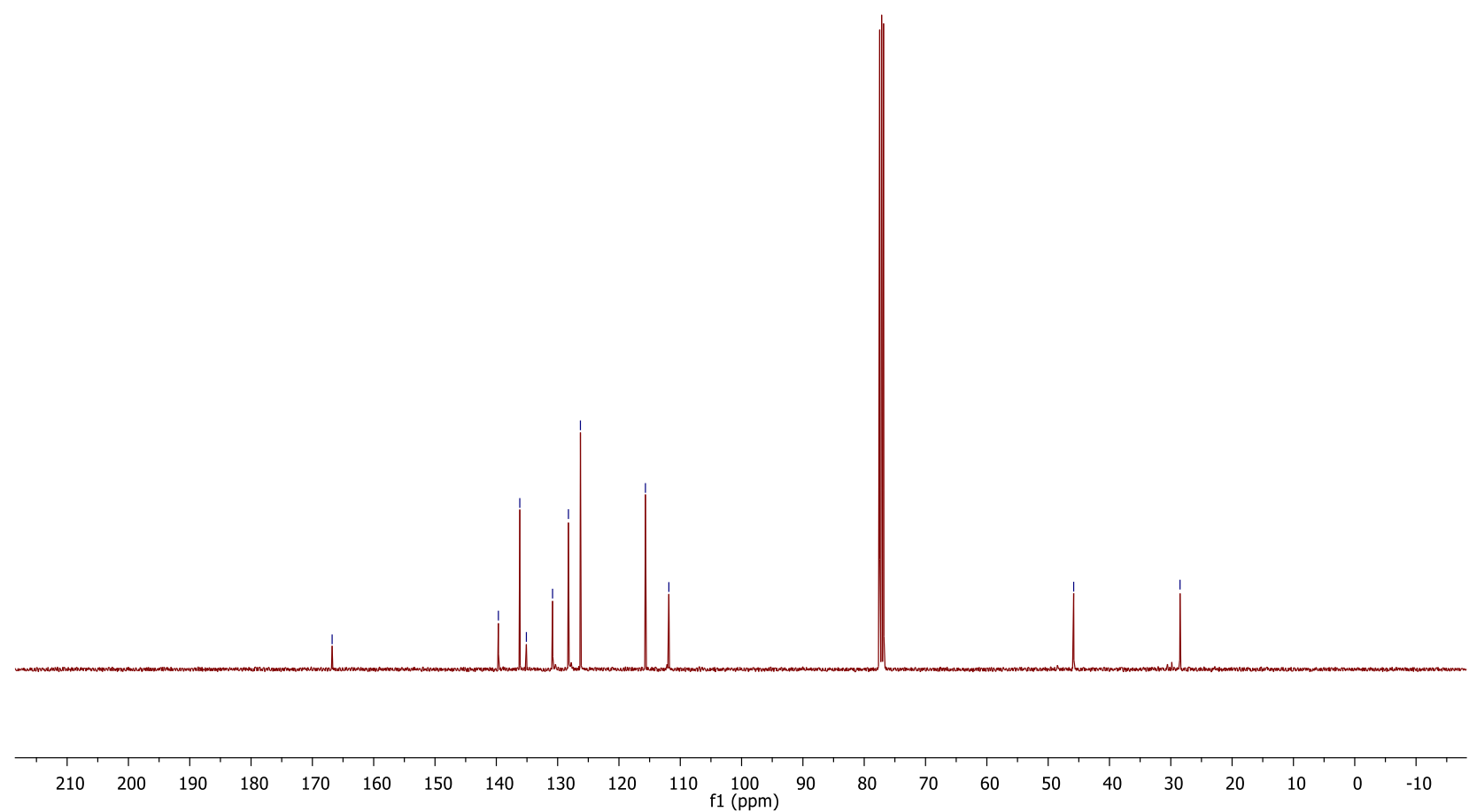


(2,3-Dihydro-1H-pyrrol-1-yl)(4-(methylthio)phenyl)methanone (4q)

踶是是

iे

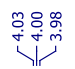

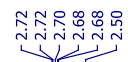

Mes

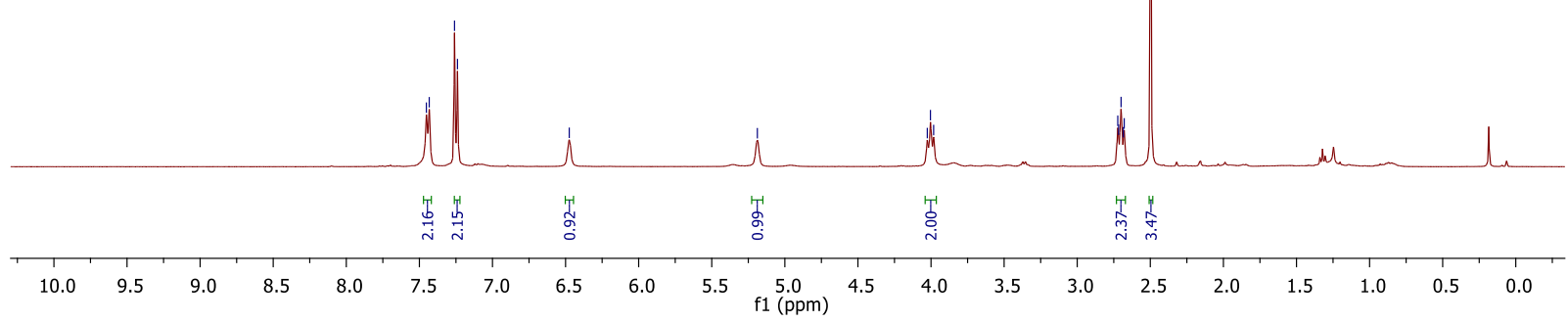

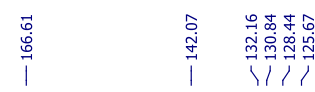

$\stackrel{\infty}{\exists}$

章
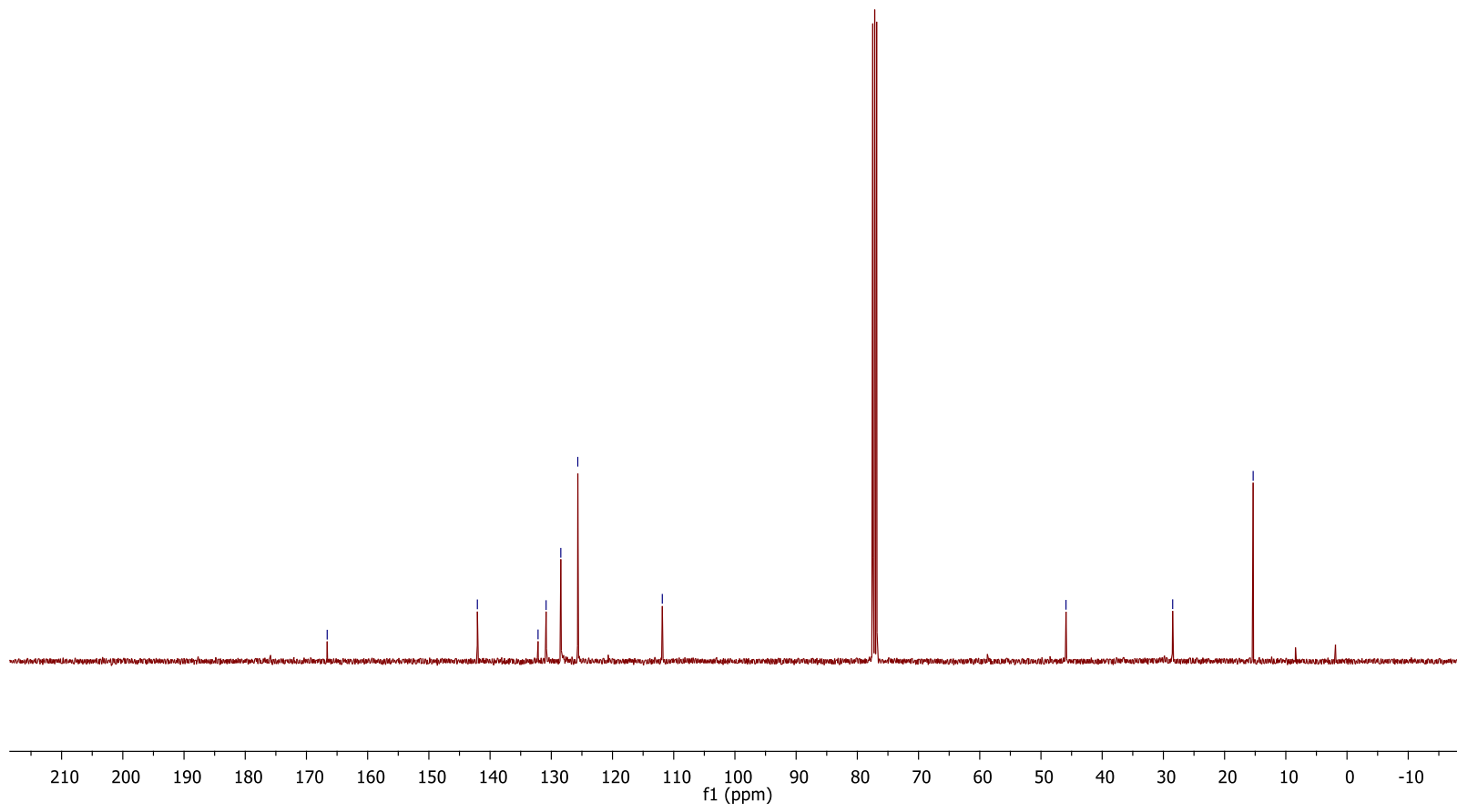

107 
3-(2,3-Dihydro-1H-pyrrole-1-carbonyl)benzonitrile (4r)

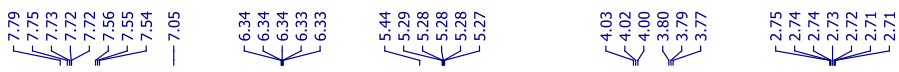<smiles>N#Cc1cccc(C(=O)N2C=CCC2)c1</smiles>

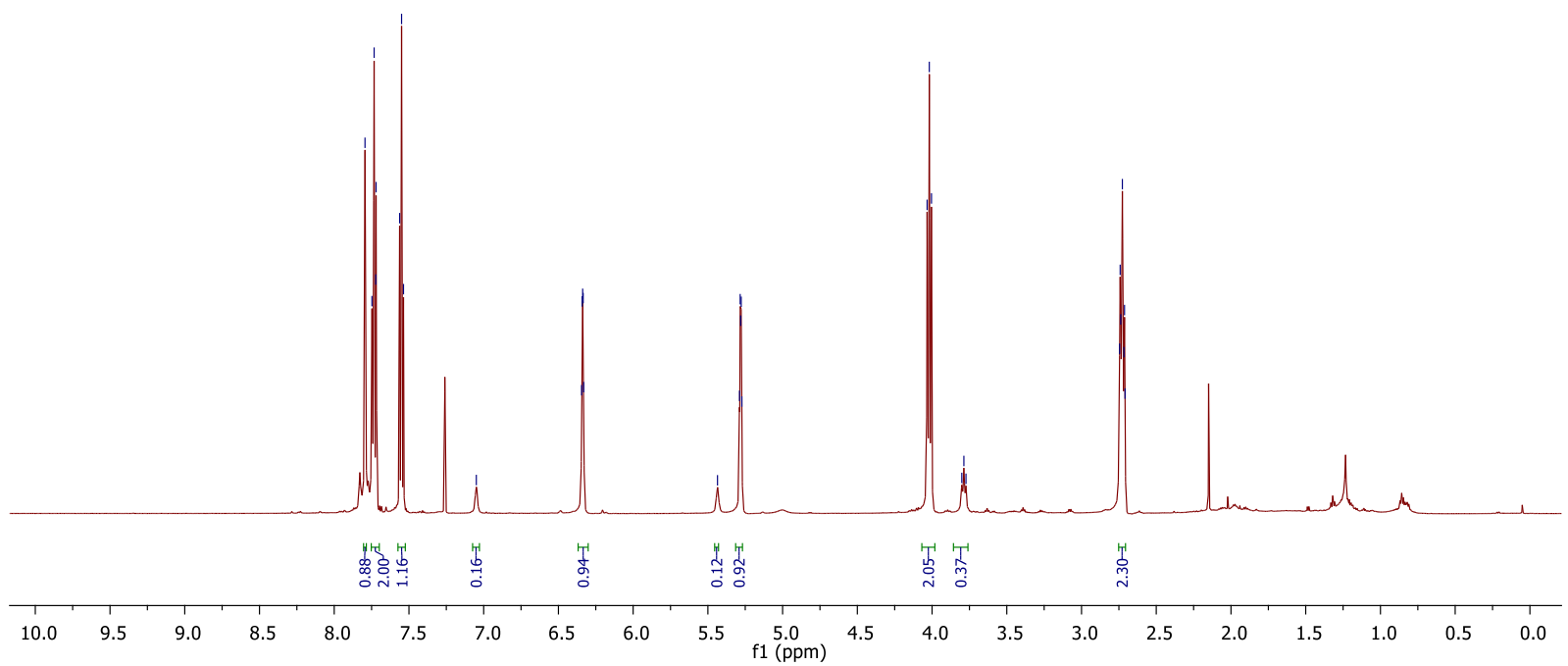

l

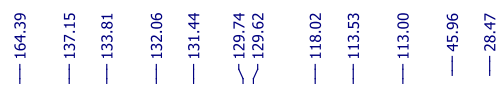
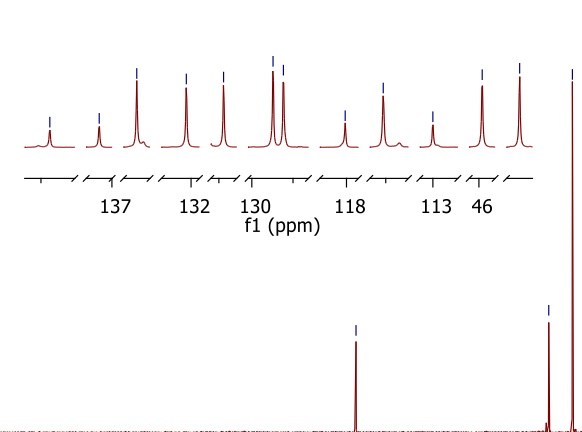

$\begin{array}{llllllllllll}210 & 200 & 190 & 180 & 170 & 160 & 150 & 140 & 130 & 120 & 110 & 100\end{array}$ 
(2,3-Dihydro-1H-pyrrol-1-yl)(ferrocene)methanone (4u)

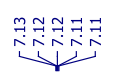

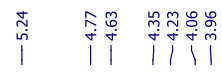

$\stackrel{\infty}{i} \underset{i}{i}$
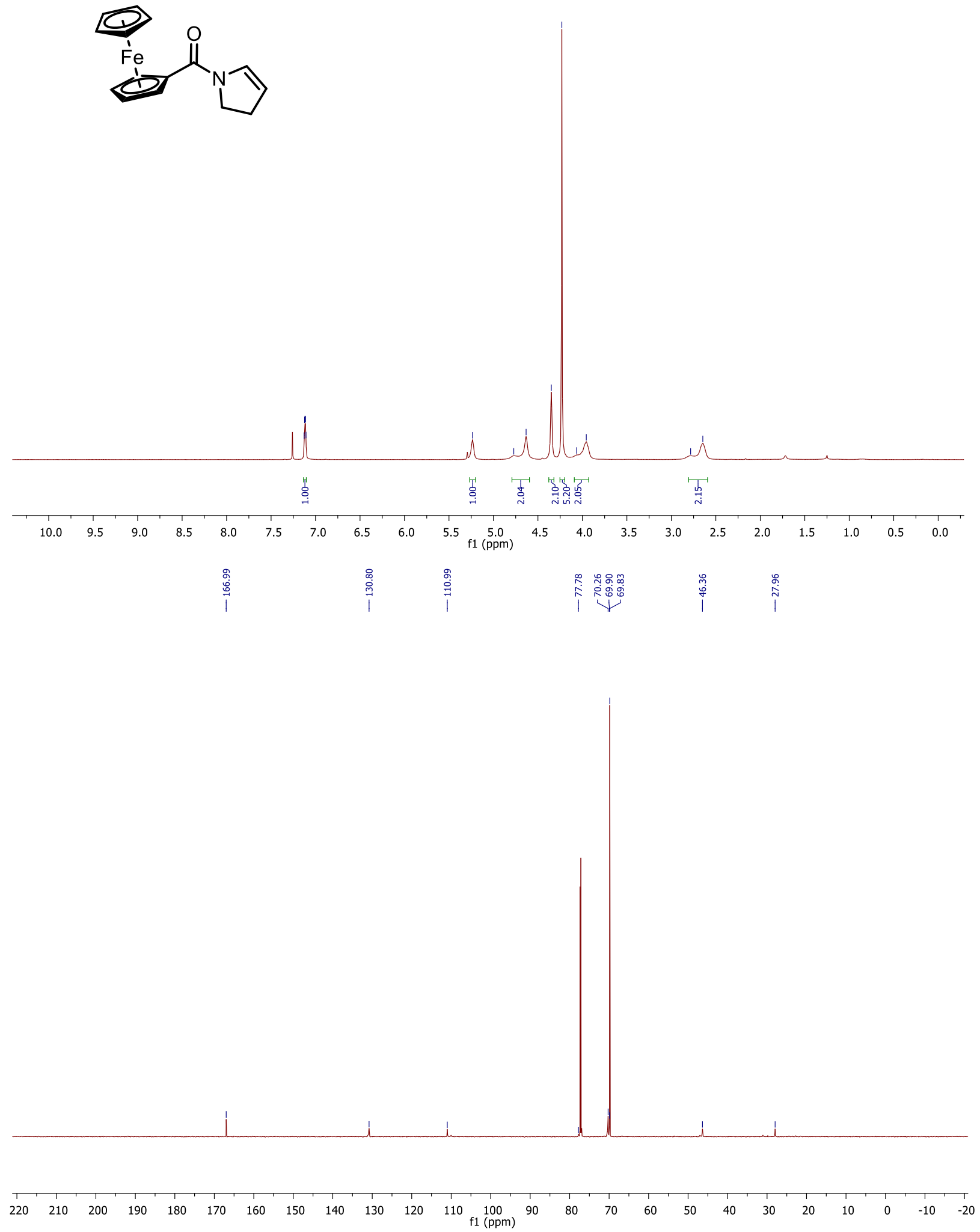

109 

methylthiazole-5-carboxylate (4v)

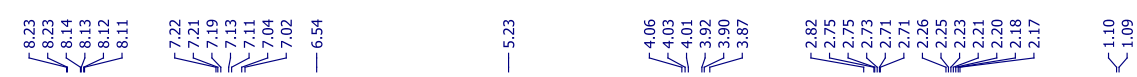<smiles>COc1cc(C(=O)N2C=CCC2)ccc1OC(=O)c1sc(-c2ccc(OCC(C)C)c(C#N)c2)nc1C</smiles>

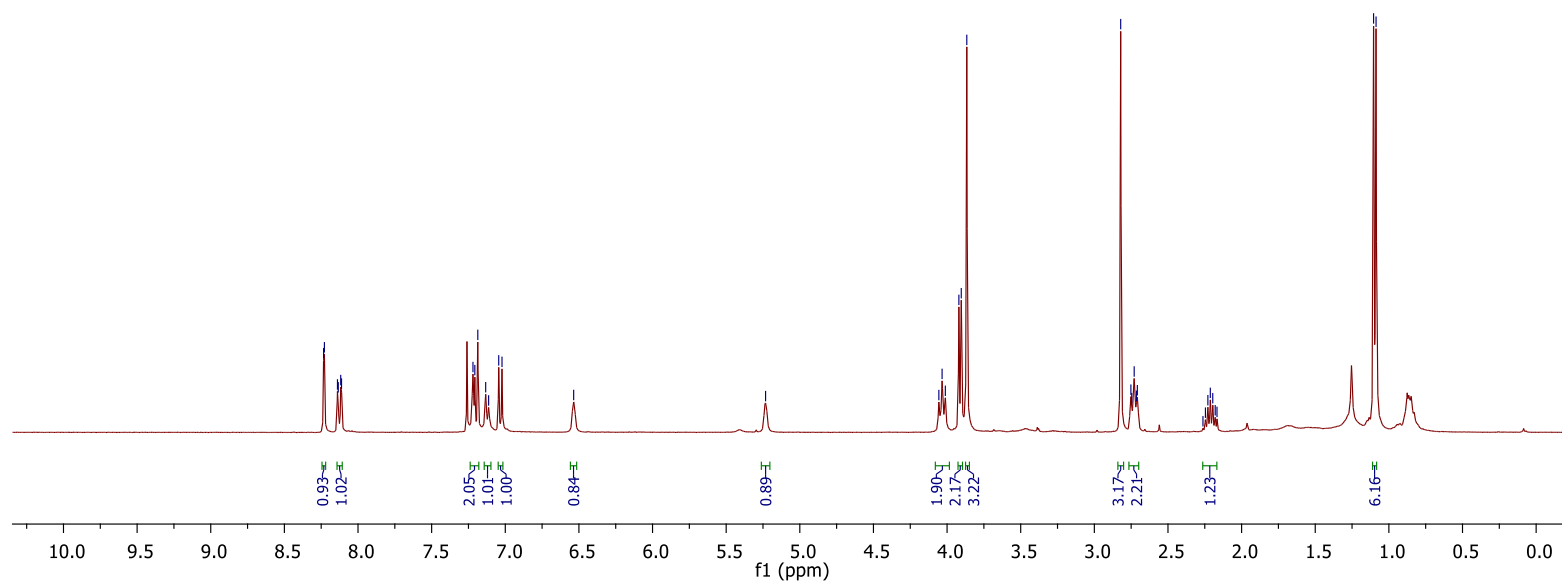

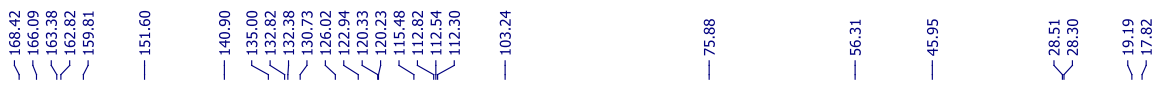


Methyl 1-benzoyl-8-oxo-2,3,3a,4,7,7a-hexahydro-1H-4,7-(epoxymethano)indole-5-carboxylate (5a)

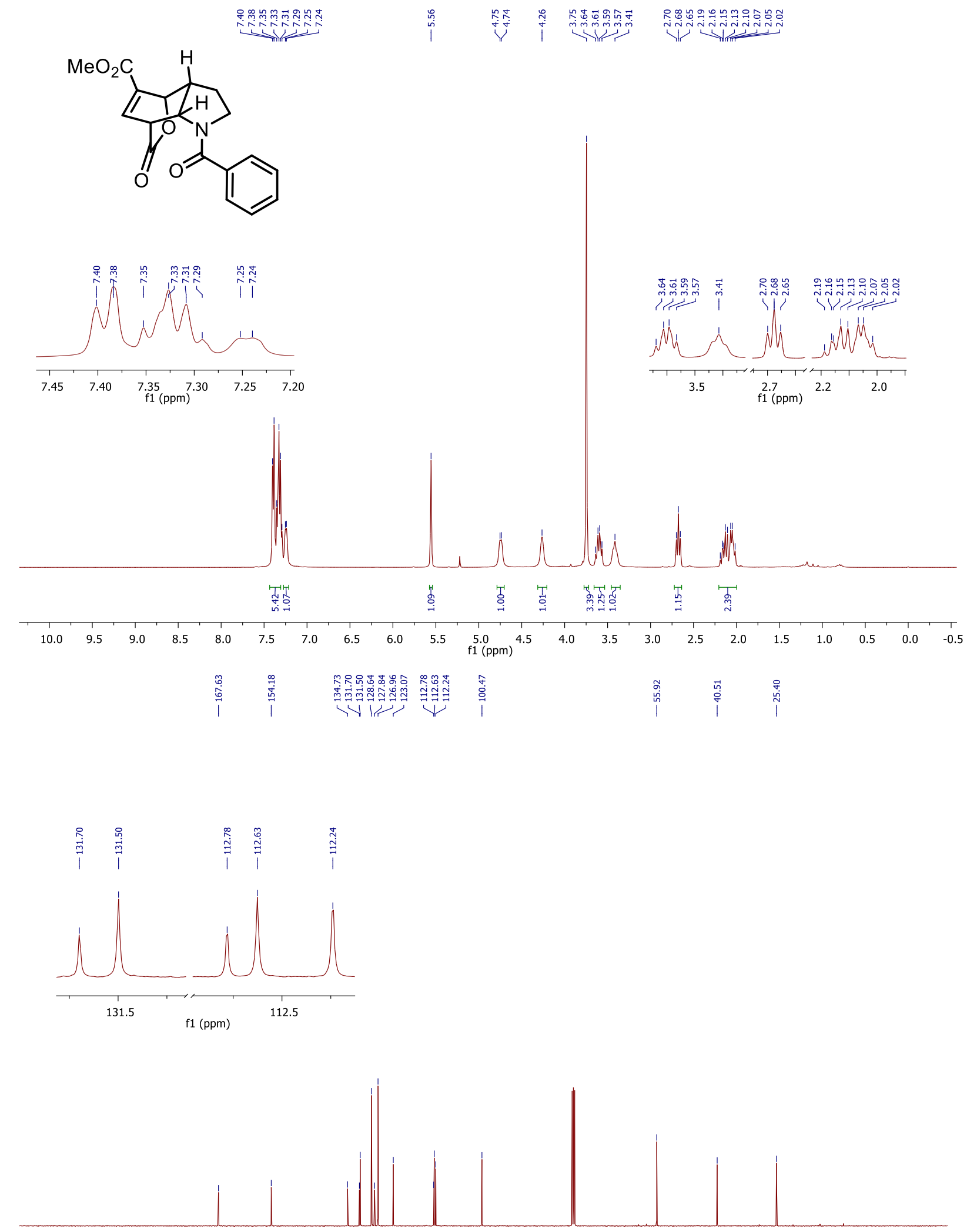

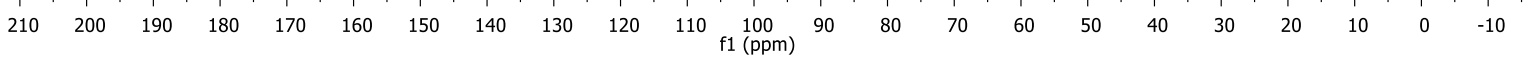


Ethyl 1-benzoyl-4-(4-chlorophenyl)-2,3-dihydro-1H-pyrrolo[3,2-c]quinoline-8-carboxylate (5b)

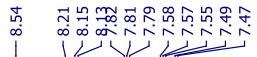

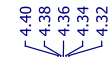
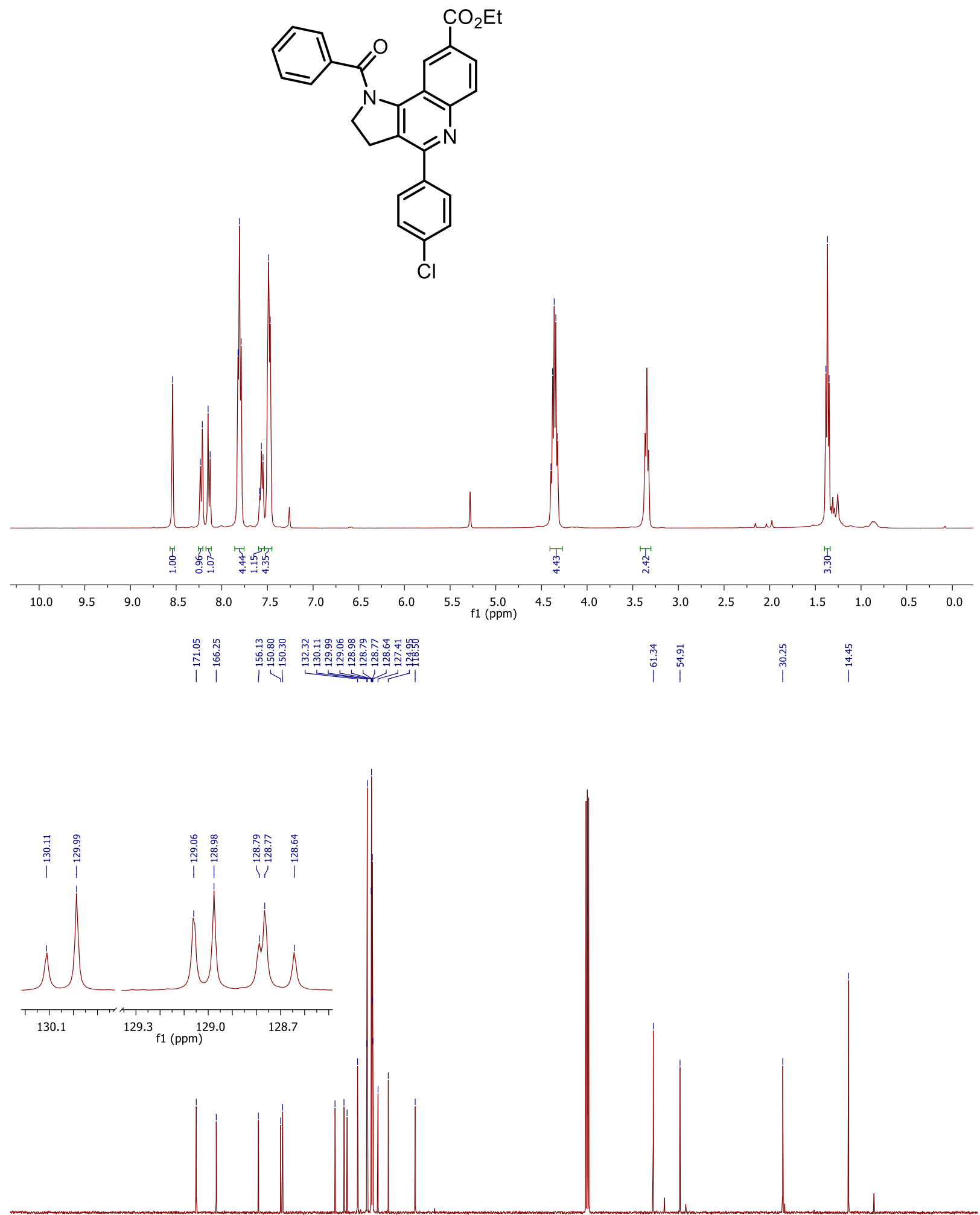

$\begin{array}{llllllllllllllllllllllllll}210 & 200 & 190 & 180 & 170 & 160 & 150 & 140 & 130 & 120 & 110 & 100 & 90 & 80 & 70 & 60 & 50 & 40 & 30 & 20 & 10 & 0 & -10\end{array}$ 


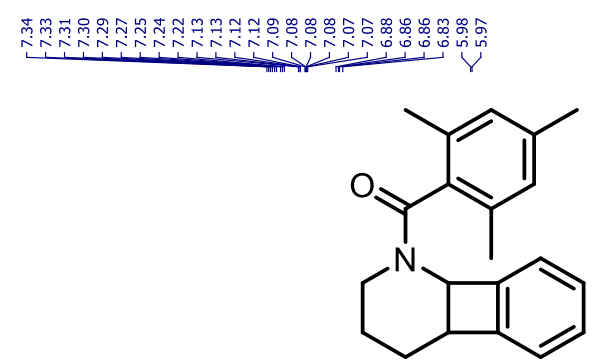

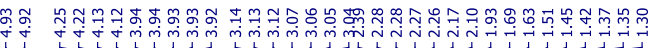
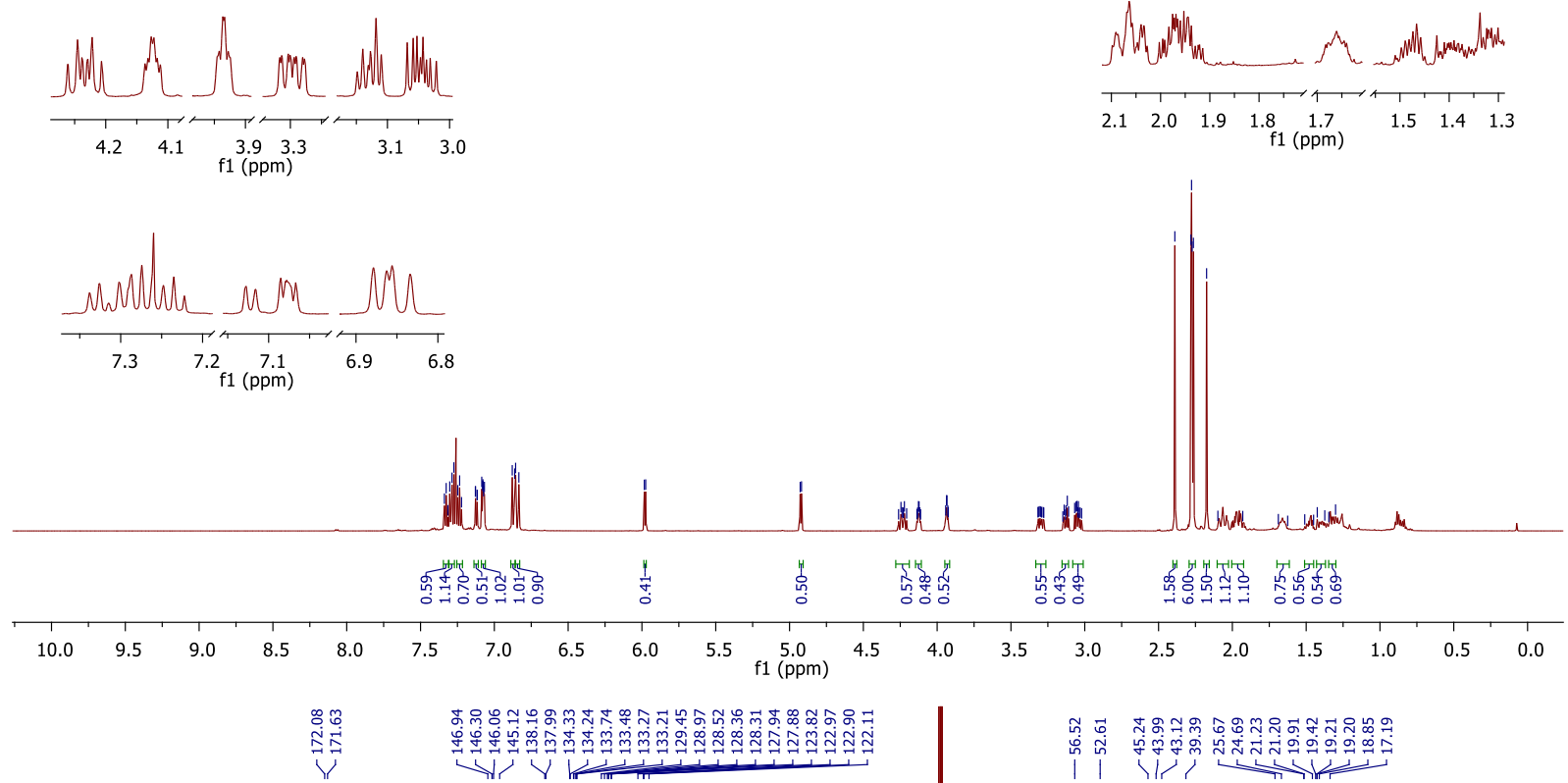

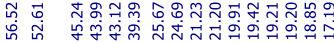

1 1 11,2

।
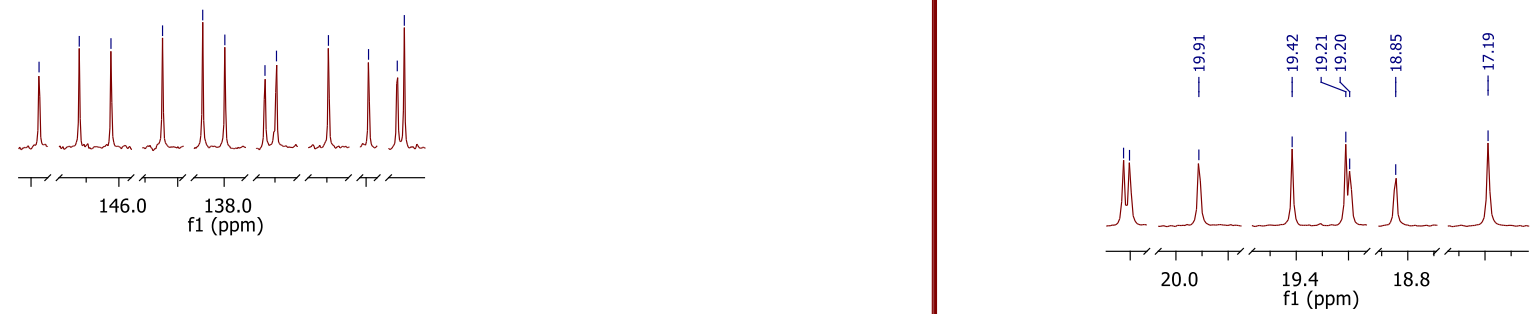

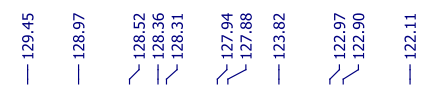
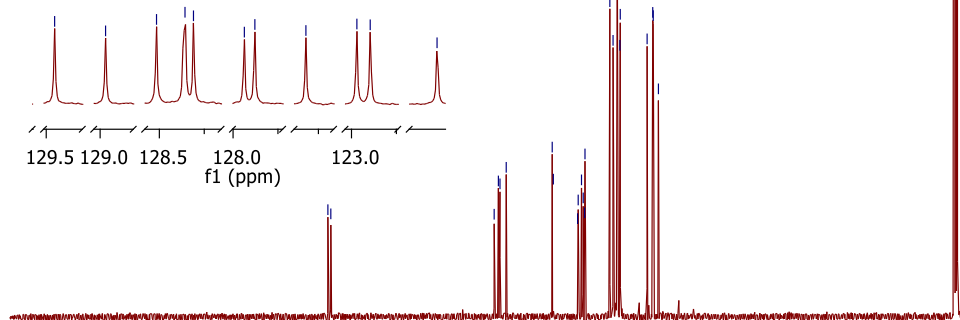

$\mathrm{f} 1(\mathrm{ppm})$

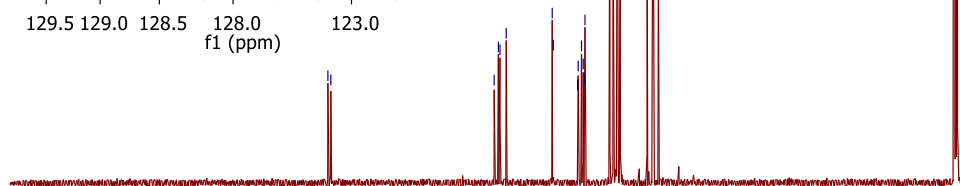


N-(2-(5-Methoxy-1H-indol-3-yl)ethyl)benzamide (5d)

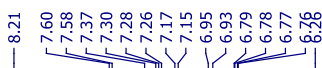<smiles>COc1ccc2[nH]cc(CCNC(=O)c3ccccc3)c2c1</smiles>

|

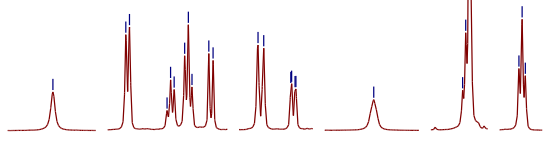

$8.07 .5 \quad \mathrm{fi}_{\mathrm{f}(\mathrm{ppm})} 6.5 \quad 3.0$

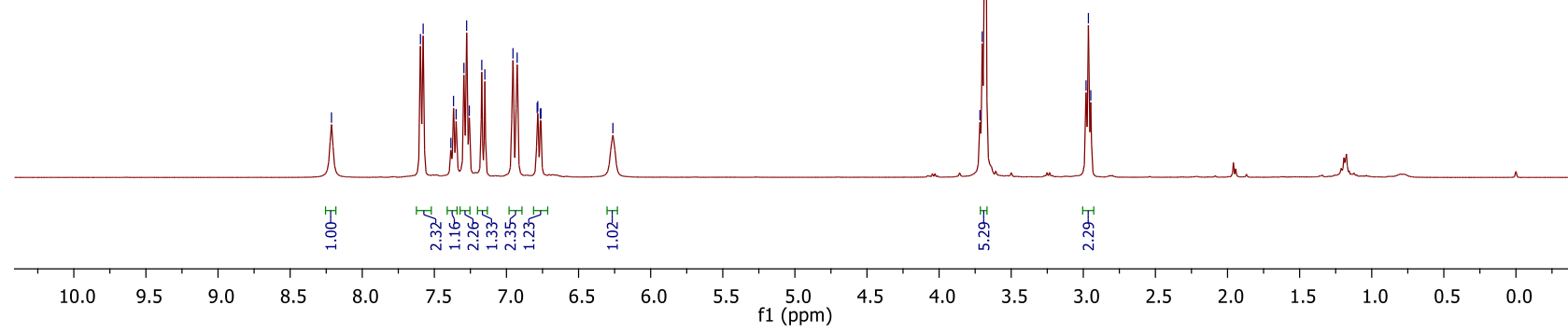

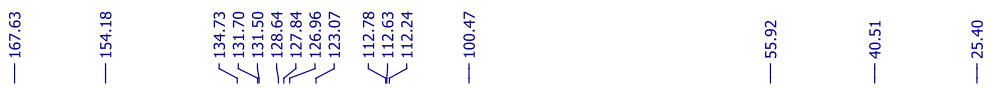

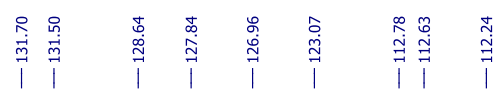

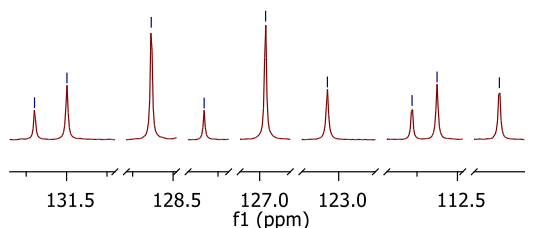

$\begin{array}{lllllllllll}210 & 200 & 190 & 180 & 170 & 160 & 150 & 140 & 130 & 120 & 110 \\ \mathrm{f} 1(\mathrm{ppm})\end{array}$ 


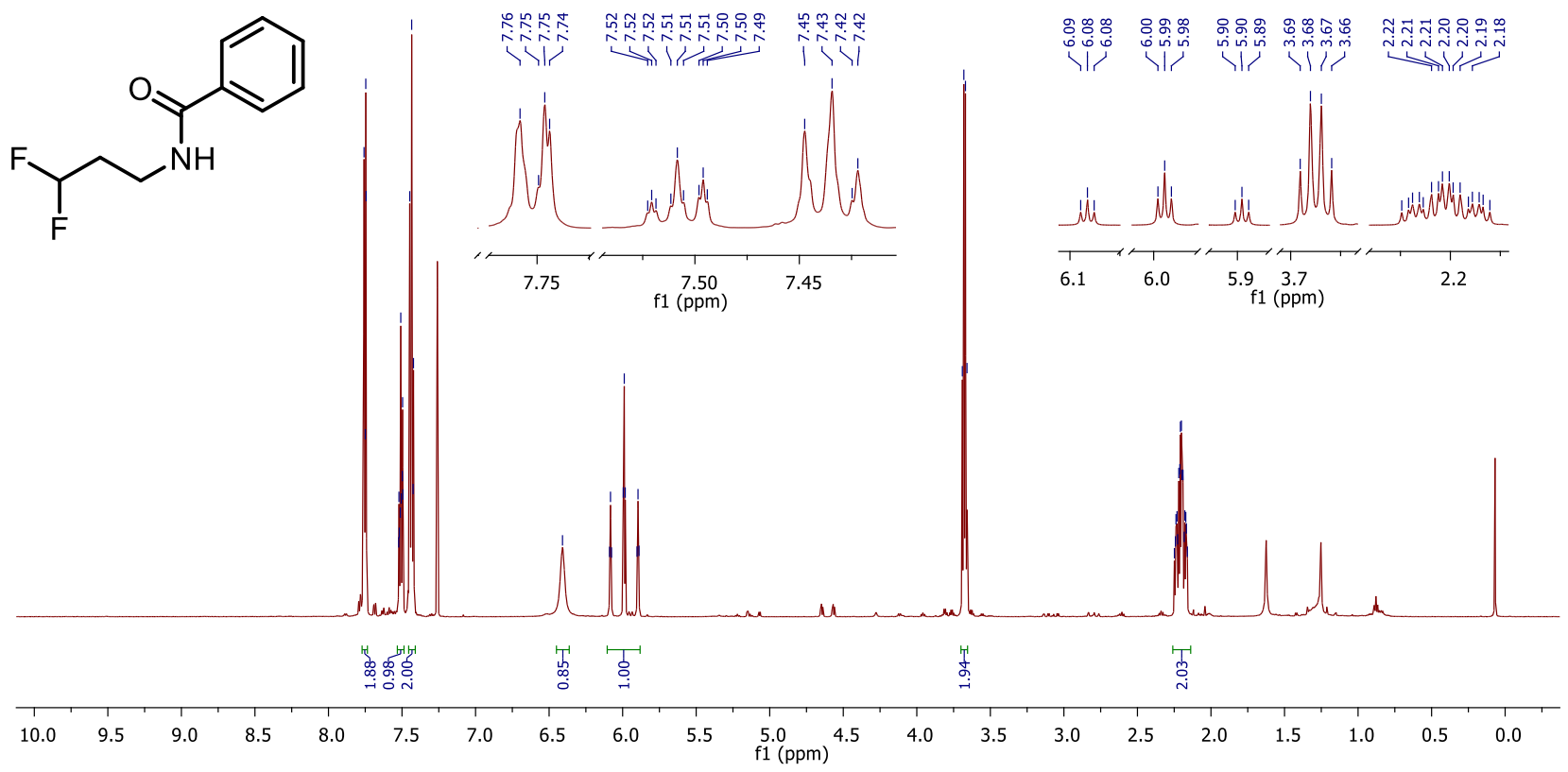

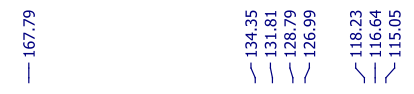

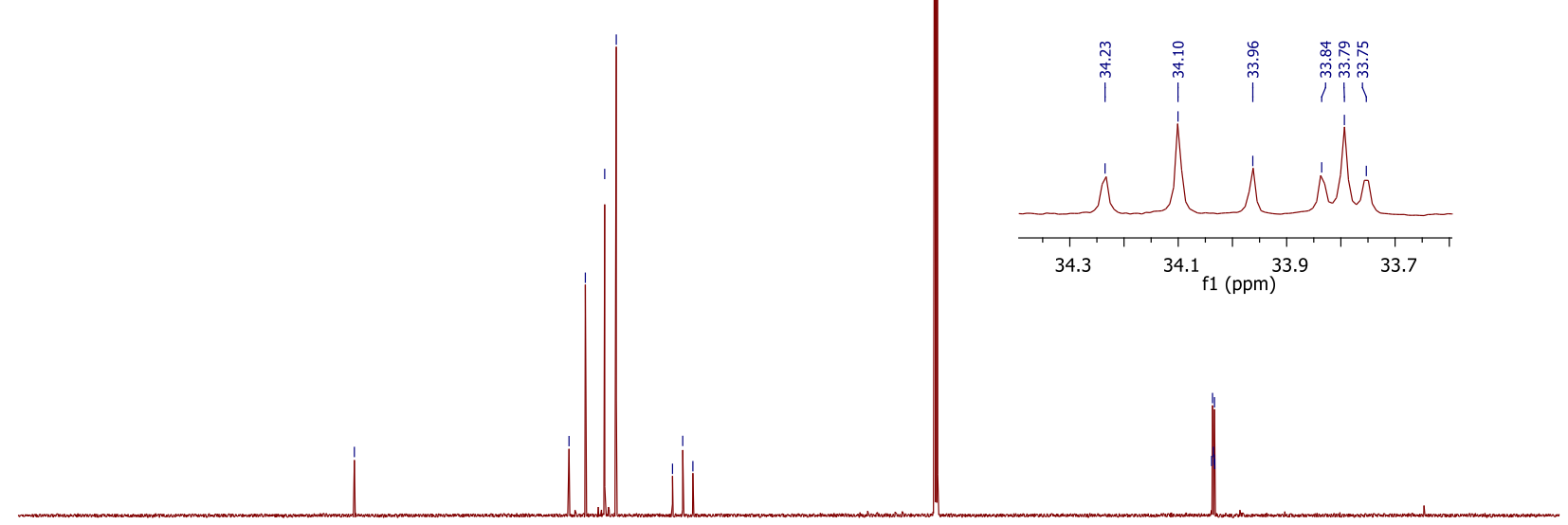

$\begin{array}{llllllllllllllllllllllllllll}220 & 210 & 200 & 190 & 180 & 170 & 160 & 150 & 140 & 130 & 120 & 110 & 100 & 90 & 80 & 70 & 60 & 50 & 40 & 30 & 20 & 10 & 0 & -10\end{array}$ 


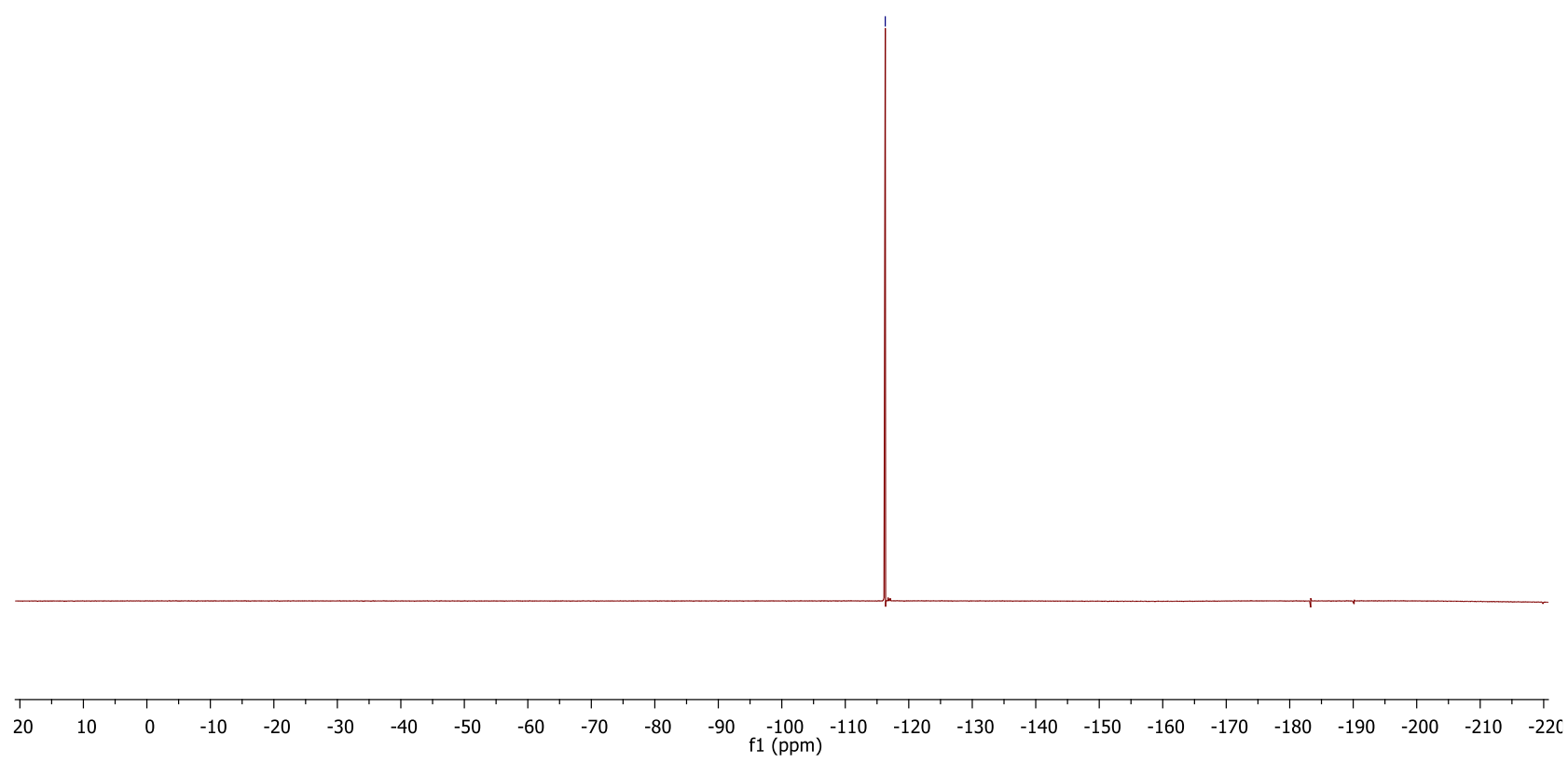


(R)-8-Hydroxy-7,9-dimethoxy-1,2,3,9b-tetrahydro-5H-pyrrolo[2,1-a]isoindol-5-one (5f)

i̊

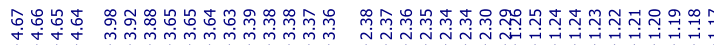

i

+
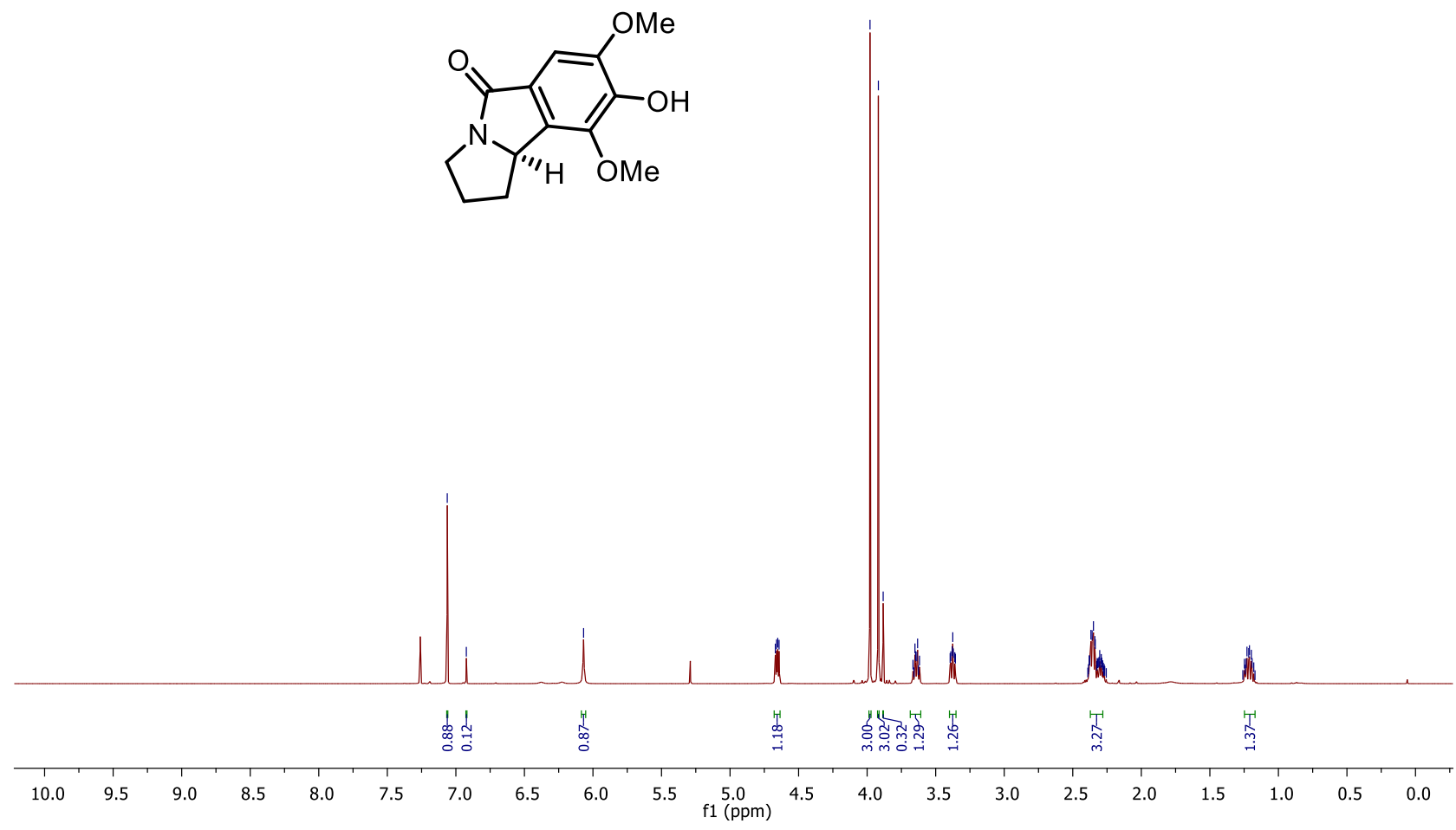

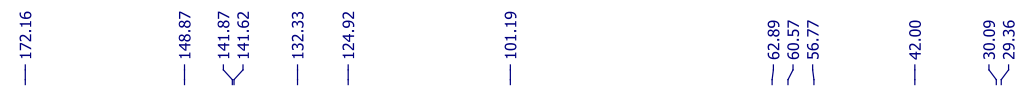

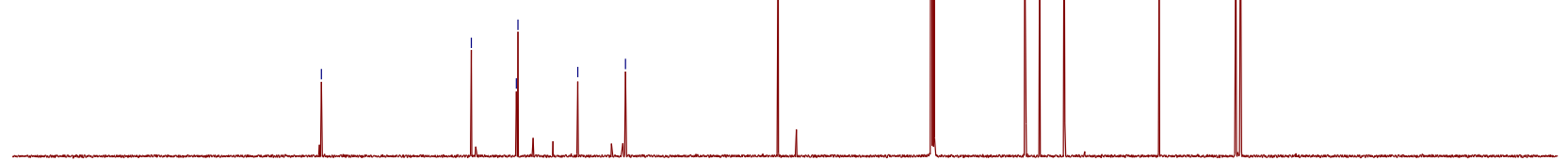

$\begin{array}{lllllllllllllllllllllllllll}220 & 210 & 200 & 190 & 180 & 170 & 160 & 150 & 140 & 130 & 120 & 110 & 100 & 90 & 80 & 70 & 60 & 50 & 40 & 30 & 20 & 10 & 0 & -10\end{array}$

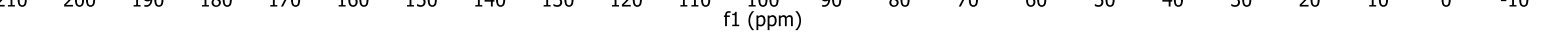


Mesityl(5-phenyl-3,4-dihydropyridin-1(2H)-yl)methanone (5g)
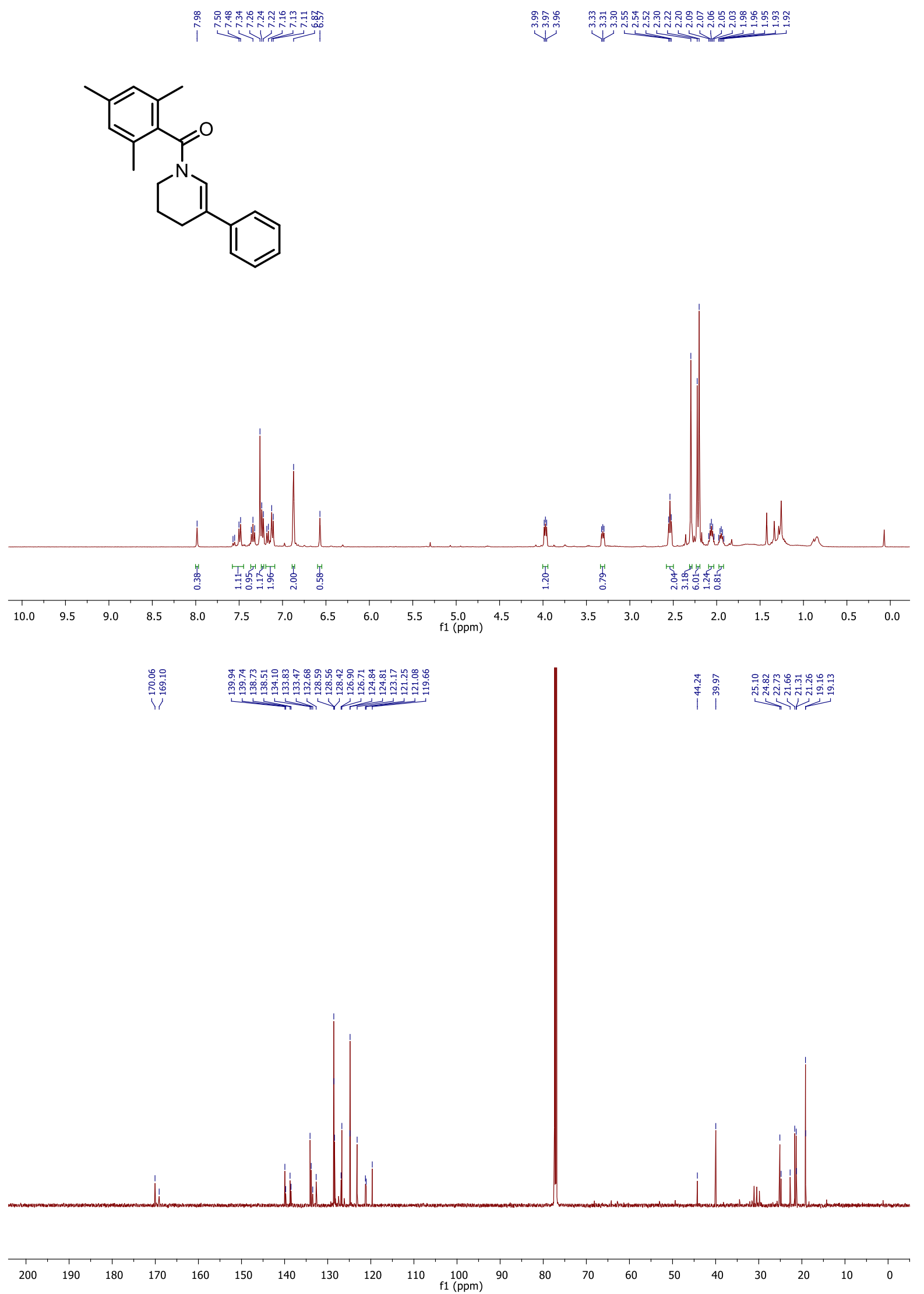


\section{X-Ray Crystallographic Data}

The X-ray intensity data was measured on Bruker D8 Venture diffractometer equipped with multilayer monochromator, Mo K/ $\alpha$ INCOATEC micro focus sealed tube and Oxford cooling system. The structure was solved by Direct Methods. Non-hydrogen atoms were refined with anisotropic displacement parameters. Hydrogen atoms were inserted at calculated positions and refined with riding model. The following software was used: Bruker SAINT software package using a narrow-frame algorithm for frame integration, $S A D A B S^{i i}$ for absorption correction, OLEX2 $2^{\text {iii }}$ for structure solution, refinement, molecular diagrams and graphical user-interface, Shelxle $e^{\text {iv }}$ for refinement and graphical user-interface SHELXS-2015 for structure solution, SHELXL-2015 vi for refinement, Platovii for symmetry check. Experimental data and CCDC-Codes Experimental data (Available online: http://www.ccdc.cam.ac.uk/conts/retrieving.html) can be found in Table 1. Crystal data, data collection parameters, and structure refinement details are given in Tables 2 to 3. Asymmetric Unit visualized in Figure 1.

Table 1 Experimental parameter and CCDC-Code.

\begin{tabular}{|c|c|c|c|c|c|c|c|c|}
\hline Sample & Machine & Source & Temp. & $\begin{array}{c}\text { Detector } \\
\text { Distance }\end{array}$ & $\begin{array}{c}\text { Time/ } \\
\text { Frame }\end{array}$ & \#Frames & $\begin{array}{c}\text { Frame } \\
\text { width }\end{array}$ & CCDC \\
\hline & Bruker & & {$[\mathrm{K}]$} & {$[\mathrm{mm}]$} & {$[\mathrm{s}]$} & & {$\left[^{\circ}\right]$} & \\
\hline $5 a$ & D8 & Mo & 100 & 30 & 10 & 500 & 0.36 & 2075992 \\
\hline
\end{tabular}




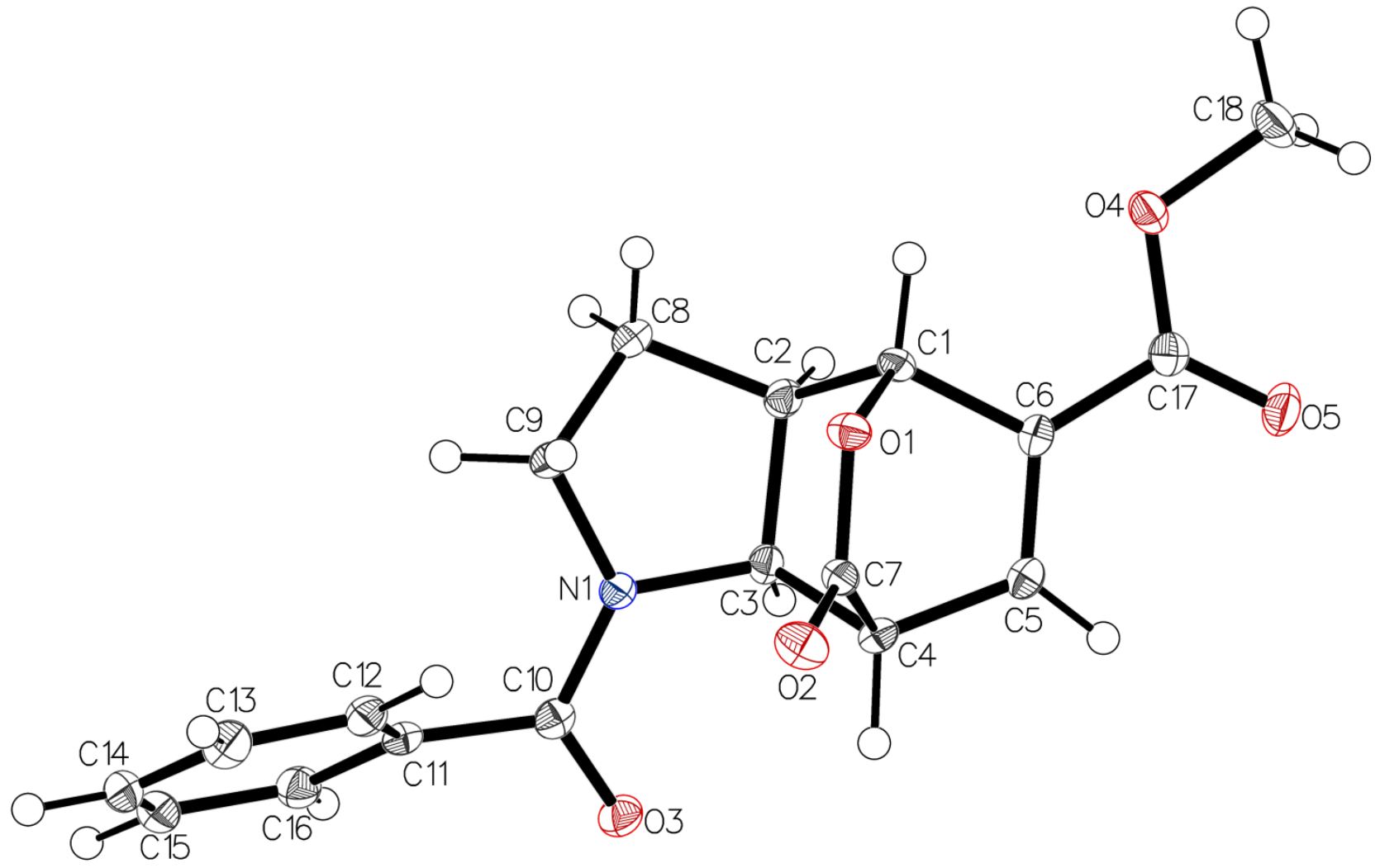

Figure 1 Crystal structure [5a] drawn with 50\% displacement ellipsoid. The bond precision for C-C single bonds is $0.0318 \AA$.

Table 2 Sample and crystal data. [5a]

\begin{tabular}{|c|c|c|c|c|c|}
\hline Radiation $[\AA]]$ & $\operatorname{MoK} \alpha(\lambda=0.71073)$ & Z & 4 & Measurement method & If and $\backslash w$ scans \\
\hline Crystal habit & clear colourless plate & $\mathrm{a}[\AA ⿻]$ & $10.5546(4)$ & & \\
\hline Crystal size $\left[\mathrm{mm}^{3}\right]$ & $0.204 \times 0.085 \times 0.011$ & $\mathrm{~b}[\AA ̊]$ & $6.4125(2)$ & Abs. correction type & multiscan \\
\hline Empirical formula & C18H17NO5 & $c[\AA]$ & $22.8672(9)$ & Abs. correction Tmin & \\
\hline Formula weight $[\mathrm{g} / \mathrm{mol}]$ & 327.32 & $\alpha\left[{ }^{\circ}\right]$ & 90 & Abs. correction Tmax & \\
\hline Temperature $[\mathrm{K}]$ & 100.0 & $\beta\left[{ }^{\circ}\right]$ & $91.479(2)$ & Density (calculated) $[\mathrm{g} / \mathrm{cm} 3]$ & 1.405 \\
\hline Crystal system & Monoclinic & $\nu\left[^{\circ}\right]$ & 90 & Absorption coefficient $\left[\mathrm{mm}^{-1}\right]$ & 0.103 \\
\hline Space group & P21c & Volume $\left[\AA^{3}\right]$ & $1547.17(10)$ & $F(000)\left[e^{-}\right]$ & 688.0 \\
\hline
\end{tabular}

Table 3 Data collection and structure refinement. [5a]

\begin{tabular}{|c|c|c|c|c|c|}
\hline $2 \Theta$ range for data collection $\left[{ }^{\circ}\right]$ & 5.186 to 61.072 & \multicolumn{2}{|c|}{ Index ranges } & Goodness-of-fit on $F^{2}$ & 1.019 \\
\hline Reflections collected & 11231 & $\mathrm{~h}$ & $-15 \leq h \leq 14$ & $\begin{array}{l}\text { Diff. peak and hole [e- } \AA^{-} \\
\text {3] }\end{array}$ & $0.34 /-0.31$ \\
\hline Data / restraints / parameters & $4567 / 0 / 218$ & $\mathrm{k}$ & $-5 \leq k \leq 9$ & & \\
\hline Refinement method & Direct Methods & I & $-32 \leq 1 \leq 32$ & Function minimized & $\Sigma w\left(F_{o}^{2}-F_{c}^{2}\right)^{2}$ \\
\hline & & all data & $\begin{array}{l}R 1=0.0848, \\
w R 2=0.1158\end{array}$ & Weighting scheme & where \\
\hline & & $1>2 \sigma(I)$ & $\begin{array}{l}R 1=0.0447 \\
w R 2=0.1037\end{array}$ & $\begin{array}{c}W=1 /\left[\sigma^{2}\left(\mathrm{Fo}^{2}\right)+\right. \\
\left.(0.0720 \mathrm{P})^{2}+204.8064 \mathrm{P}\right]\end{array}$ & $\mathrm{P}=\left(\mathrm{F}_{\mathrm{o}}^{2}+2 \mathrm{~F}_{\mathrm{c}}^{2}\right) / 3$ \\
\hline
\end{tabular}




\section{References}

'Bruker SAINT v8.38B Copyright (C) 2005-2019 Bruker AXS

ii Sheldrick, G. M. (1996). SADABS. University of Göttingen, Germany.

iii Dolomanov, O.V., Bourhis, L.J., Gildea, R.J, Howard, J.A.K. \& Puschmann, H. , OLEX2, (2009), J. Appl. Cryst. 42, 339-341

iv C. B. Huebschle, G. M. Sheldrick and B. Dittrich, ShelXle: a Qt graphical user interface for SHELXL, J. Appl. Cryst., 44, (2011) 1281-1284

` Sheldrick, G. M. (2015). SHELXS v 2016/4 University of Göttingen, Germany.

vi Sheldrick, G. M. (2015). SHELXL v 2016/4 University of Göttingen, Germany.

${ }^{\text {vii } A . ~ L . ~ S p e k, ~ A c t a ~ C r y s t . ~ 2009, ~ D 65, ~ 148-155 . ~}$ 